UniversidAd PolitéCNICA DE MADRID

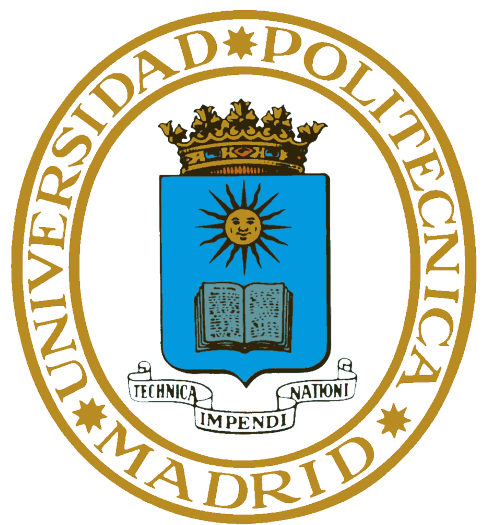

ESCUELA TÉCNICA SUPERIOR DE INGENIEROS INFORMÁTICOS

\title{
Cryptographic Techniques for the Security of Cloud and Blockchain Systems
}

PH.D THESIS

Ph.D. Candidate

Luca Nizzardo

Academic Advisor

Dario Fiore

Research Assistant Professor

IMDEA Software Institute 
Copyright@2018 by Luca Nizzardo 
DEPARTAMENTO DE LENGUAJES Y SISTEMAS INFORMÁTICOS E INGENIERIA DE SOFTWARE

ESCUELA TÉCNICA SUPERIOR DE INGENIEROS INFORMÁTICOS

\title{
Cryptographic Techniques for the Security of Cloud and Blockchain Systems
}

\author{
SUBMITTED IN PARTIAL FULFILLMENT OF THE REQUIREMENTS FOR THE DEGREE OF: \\ Doctor of Philosophy in Software, Systems and Computing
}

Author: Luca Nizzardo

Advisor

Dario Fiore

2018 
Tribunal:

Presidente: Prof. Manuel Hermenegildo Salinas, Universidad Politécnica de Madrid, Spain

Secretario: Prof. María Isabel González Vasco, Universidad Rey Juan Carlos, Madrid, Spain

Vocal: Prof. Bogdan Warinschi, University of Bristol, United Kingdom

Vocal: Prof. Georg Fuschbauer, Ecole Normale Superieure, Paris, France

Vocal: Dr. Claudio Soriente, NEC Europe, Germany

Suplente: Prof. Boris Köpf, IMDEA Software Institute, Madrid, Spain

Suplente: Dr. Ángel Pérez del Pozo, Universidad Rey Juan Carlos, Madrid, Spain 


\section{Resumen de la Tesis Doctoral}

Las interacciones sociales normalmente involucran personas con distintos intereses, incluso intereses contradictorios en algunas ocasiones, como por ejemplo, relaciones entre compradores y vendedores o entre consumidores y servicios. Tradicionalmente estas interacciones han tenido lugar en persona y la sociedad ha desarrollado durante años diferentes formas de proteger a los usuarios frente a un comportamiento malicioso por alguna de las partes. Sin embargo, en la era digital, cada vez es más común que estas interacciones tengan lugar a través de Internet, donde la gente no se reúne en persona o incluso no se conoce. Proteger a los usuarios en este escenario es mucho más complicado y requiere herramientas digitales adicionales.

En esta tesis se investiga cómo conseguir que las distintas partes de una interacción digital se comporten de forma honesta. En particular, se consideran dos problemas emergentes en este ámbito: computación en la Nube y comercio electrónico.

A través de un servicio en la Nube, cualquiera (un Cliente) puede enviar su información y datos a un servidor remoto (la Nube) y después pedir a la Nube que realice operaciones sobre dicha información. Este nuevo modelo permite un gran ahorro en tiempo, poder de computación y otros costes infraestructurales para el usuario, ya que puede subcontratar estos servicios. Este modelo proporciona una funcionalidad muy prometedora, pero, al mismo tiempo, introduce la necesidad de confiar en la Nube, lo que puede llegar a ser problemático por diversos motivos. Los dos principales problemas que más ocupan a la comunidad recientemente son el de privacidad y el de autenticidad de la información almacenada y procesada en entornos no confiables. Intuitivamente, la privacidad es importante porque un cliente no desea que el servidor obtenga ninguna información sobre los datos que le ha proporcionado. Por otro lado, los Clientes necesitan autenticidad para asegurarse de que la Nube ha calculado correctamente las operaciones que han sido pedidas. Esta tesis se centra en el segundo problema: estudiar y mejorar los 
autenticadores homomorficos.

Los autenticadores homomórficos permiten que un Cliente, $\mathcal{C}$, almacene informacion autenticada en la Nube. Más tarde, una tercera parte (el Verificador) puede pedirle a la Nube que calcule una función, $f$, sobre la información de $\mathcal{C}$. Usando un procedimiento especial, la Nube puede convencer al Verificador de que la computación se ha realizado correctamente, a través de una pieza de información llamada autenticador. La contribución de esta tesis consiste en mejorar tres aspectos sobre los autenticadores homomórficos: definiciones, eficiencia y funcionalidad. Primero, se introduce un nuevo modelo de seguridad que es más exigente y fácil de manejar comparado con el modelo anterior. Además, se proponen dos compiladores que permiten pasar del modelo anterior al nuevo. Segundo, se presenta el primer esquema de firma linealmente homomórfico con una clave de verificación de tamaño sub-lineal en el tamaño del conjunto de datos. Tercero, se formaliza la noción de autenticadores homomórficos para funciones que toman entradas autenticadas por diferentes partes (usando claves diferentes) y se presentan construcciones contretas tanto para verificación pública como privada.

En cuanto al comercio electrónico, y más generalmente, la posibilidad de transferir valores a través de Internet, la invención del Bitcoin [100] ha resultado una revolución, ya que es el primer protocolo que permite a sus usuarios transferir valores digitales sin necesidad de una autoridad de confianza.

Además de su utilidad y potencial en el ámbito de pagos y transacciones, Bitcoin se ha hecho famoso por la tecnología en la que se basa: la Blockchain, que se podría describir como un registro distribuido sobre el cual los usuarios se ponen de acuerdo y donde todas las transacciones son registradas de forma inalterable. Esta peculiar característica ha hecho que el paradigma de Blockchain se convirtiera en uno de los más populares y prometedores conceptos en los últimos años.

Además, se le ha dado mucha importancia y atención a las posibilidades que ofrece la tecnología de Blockchain más allá de Bitcoin. En concreto, este trabajo se centra en alcanzar un modelo de "intercambio equitativo" a partir de la tecnología de Blockchain, donde el objetivo es que dos usuarios intercambien bienes digitales de forma que ninguno pueda engañar al otro.

En este sentido, se considera un protocolo introducido por Maxwell [94] conocido como "Pagos Contingentes de Conocimiento Cero" (ZKCP). La contribución de esta tesis es doble: Primero se identifica y describe un fallo en el protocolo de ZKCP existente y se propone cómo se podría solucionar de forma eficiente, modificando el protocolo original. Segundo, se argumenta cómo este nuevo protocolo proporciona funcionalidades adicionales, que eran imposibles de alcanzar antes. 


\section{Abstract of the Dissertation}

Human interactions often involve people who have different and sometimes contrasting interests, like buyers and sellers or consumers and providers. For what regards physical interactions, the society has developed during the years many different ways to protect users against misbehaviors. Nevertheless, when this communication happens in the digital world through the Internet, where people do not meet or even know each other, such a protection is more challenging to obtain, and additional digital tools are needed in order to defend users.

In this thesis we investigate how to enforce the honest behavior of parties involved in a digital interaction over the Internet. In particular, we consider two emerging paradigms in this setting: Could computing and E-commerce.

By using a Cloud service, anyone (a Client) can outsource data to a remote server (the Cloud), and later on ask the Cloud to perform operations on them. This new paradigm allows for huge potential savings in terms of time, computing power and other kind of infrastructural costs, since it is possible to access to pay-per-use services upon request. It is clear that a computing paradigm like this is very attractive but, at the same time, trusting the Cloud can be problematic for various reasons. Two main security concerns that have been recently given attention by the research community are those about the privacy and authenticity of the data stored and processed in untrusted environments. Intuitively, for privacy a Client does not want the server to learn any information about the outsourced data. For authenticity, the Client instead wants to be sure that the Cloud computed correctly on the outsourced data. In this thesis we focus on this second problem, advancing the study of homomorphic authenticators.

In homomorphic authenticators a Client $\mathcal{C}$ outsources authenticated data to the Cloud. Later on, a third entity (the Verifier) can ask the Cloud to compute a function $f$ over the Client's outsourced data. Using a special procedure, the Cloud can provide the Verifier with an authenticator for the output of the function, which allows the Verifier to check the validity of the computation queried. 
The contribution of this thesis addresses three different aspects of homomorphic authenticators: definitions, efficiency and functionalities. First, it introduces a new security model which is stronger and easier to deal with compared with the existing one, along with two compilers which allow one to go from the old model to the new one. Second, it provides the first linearly homomorphic signature scheme whose verification keys have size sublinear in that of the outsourced dataset. Third, it formalizes the notion of homomorphic authenticators for functions which take inputs authenticated using different keys, providing concrete constructions both in the case of private and public verification.

For what regards E-commerce and, more in general, the possibility of transferring value through the Internet, a breakthrough result was the invention of Bitcoin [100], the first protocol which allows users to transfer digital value without relying on any trusted authority.

Other than being appealing for its potential in the framework of payments, Bitcoin became also famous for its underlying technology, the Blockchain, that can be described as a distributed ledger over which all the users can reach consensus, and where all the transactions are recorded in an immutable way. This peculiar feature made the "Blockchain paradigm" to become one of the most popular and promising concepts in the last few years.

In particular, a lot of attention has been devoted to the possible uses of the Blockchain technology beyond Bitcoin. Specifically, this work is focused on achieving fair exchange by profiting of the Blockchain features, where with fair exchange we mean the possibility for two users to swap digital goods such that neither can cheat the other.

In this area we consider a known protocol introduced by Maxwell [94] and known as Zero Knowledge Contingent Payments (ZKCP). The contribution of this thesis is twofold: first, it identifies a flaw in the ZKCP and proposes how to efficiently solve the security issue by changing the original protocol. Second, it shows that this new protocol supports additional functionalities which were impossible to achieve before. 
To Zita, for her endless love and support 


\section{Acknowledgments}

The first person I would like to thank is my wife Zita, for her endless love, patience and support. Essentially, for sharing with me everything in the last ten years. I am really grateful and I feel really lucky for that.

Thanks a lot also to my advisor Dario Fiore, for his passion, his patience and his guidance during the last four years. This thesis would not have been possible without all the fruitful discussion I had with him, profiting by his knowledge, kindness and support. I also want to thank my office mate Miguel: my $\mathrm{PhD}$ would not have been that funny without him around. Apart from that, I really enjoyed sharing opinions and research directions with him during the last four years. I will be always grateful also to Rosario Gennaro for multiple reasons: for giving me the first opportunity to approach cryptography by hosting me at City College back in 2013, and later on for allowing me to join his group in Spring 2017. While in the US, I also really enjoyed working with Steven Goldfeder and Matteo Campanelli, from whom I learnt a lot.

I would also like to thank all the "IMDEA people", and of course my parents and my brother who have been supporting and encouraging me from Italy. 
List of Publications xi

\begin{tabular}{lll}
\hline & Introduction & 1
\end{tabular}

1.1 Our Contribution . . . . . . . . . . . . . . . . . . . . 2

1.2 Authenticated Computations in the Cloud . . . . . . . . . . . . 3

1.2.1 New Security Notions for Homomorphic Signatures . . . . 5

1.2.2 Shorter Public Keys via Asymmetric Programmable Hash Functions .................... 7

\begin{tabular}{lll}
\hline 1.2 .3 & Homomorphic Authenticators Dealing With Multiple Keys & 7
\end{tabular}

1.3 Fair Exchange Over The Bitcoin Blockchain . . . . . . . . . . . 8

1.3 .1 Zero Knowledge Contingent Payments . . . . . . . . . . . 9

1.3.2 Zero Knowledge Contingent Payments for Services: Fixing and Extending ZKCP . . . . . . . . . . . . . . . 11

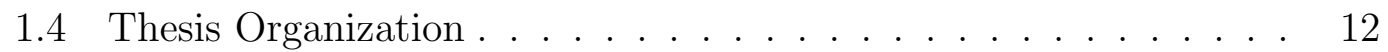

2 Improving Definitions: A New Framework for Homomorphic Signatures 13

2.1 An Overview of Our Contribution . . . . . . . . . . . . . . . . . 13

2.2 Related work . . . . . . . . . . . . . . . . . . . . . . . . . 16

2.3 An Overview of Our Compiler . . . . . . . . . . . . . . . . . 16

2.4 Defining Homomorphic Signatures . . . . . . . . . . . . . . . . . . 17

2.4 .1 Security Definitions . . . . . . . . . . . . . . 20

2.5 Generic Transformations from Semi-Adaptive to Strong Adaptive

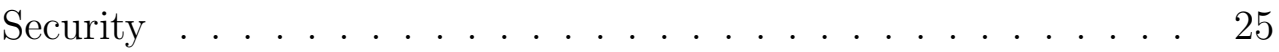

2.5.1 Strong Adaptive Security from OR-Homomorphic Signatures 26

\begin{tabular}{|ll|}
\hline 2.5 .2 & Strong Adaptive Security from Linearly-Homomorphic Sig- \\
\hline natures & 31
\end{tabular} 


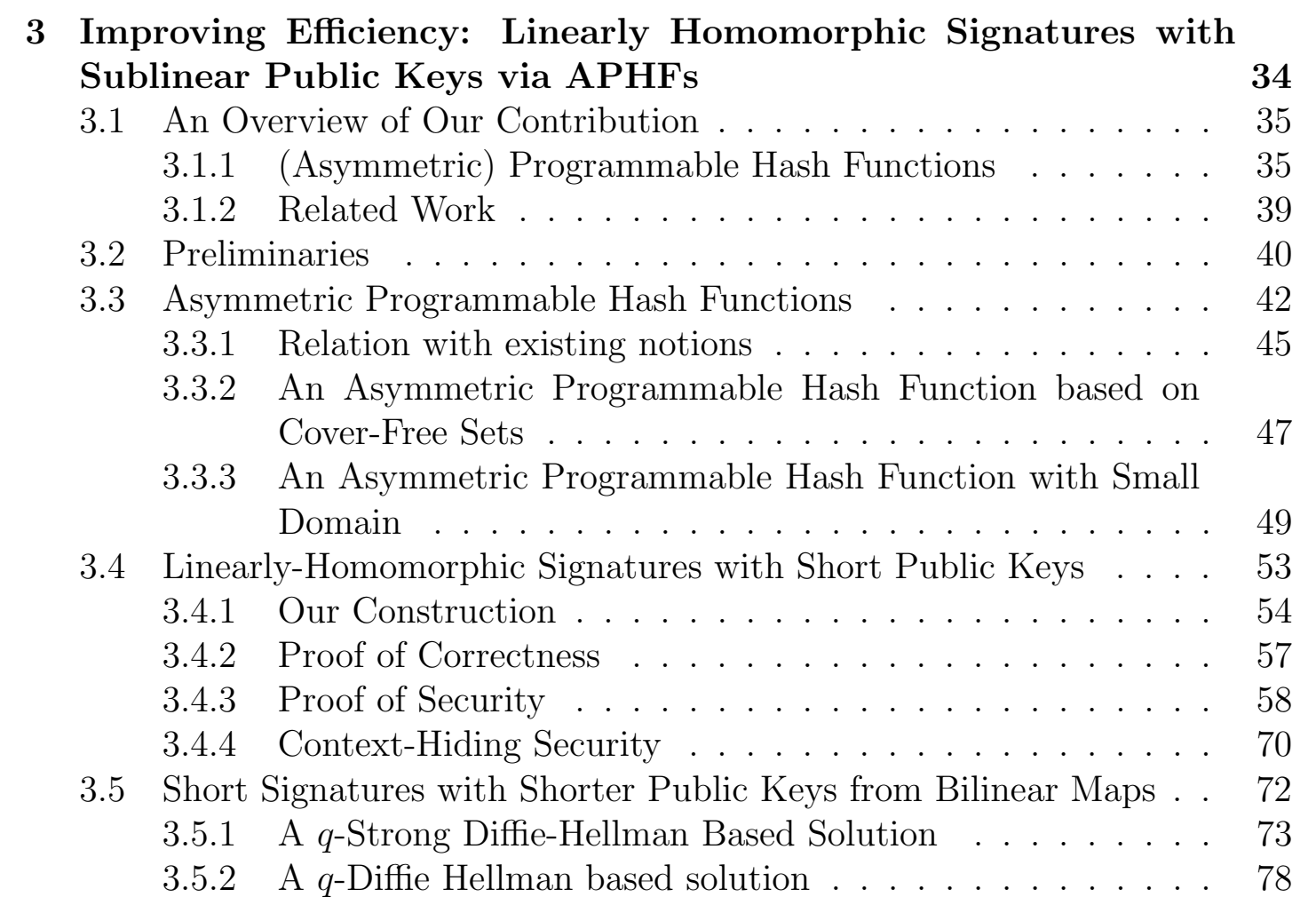

4 Extending Functionalities: Multi-Key Homomorphic Authenticators 82

4.1 On the Concept of Multi-Key Homomorphic Authenticators . . . 82

4.2 An Overview of Our Contribution . . . . . . . . . . . . . . . . . 83

4.2 .1 Related Work . . . . . . . . . . . . . . . . . . 84

4.3 Preliminaries $\ldots \ldots \ldots \ldots$. . . . . . . . . . . . . . . . . 86

4.4 Multi-Key Homomorphic Authenticators . . . . . . . . . . . . . . 87

$4.4 .1 \quad$ Non-Adaptive Corruption Queries . . . . . . . . . . . . . . 92

$4.4 .2 \quad$ Weakly-Adaptive Secure multi-key HAs . . . . . . . . . . . . . 93

4.5 Our Multi-Key Fully Homomorphic Signature . . . . . . . . . . . . 94

$4.5 .1 \quad$ Lattices and Small Integer Solution Problem . . . . . . . . 94

4.5.2 Our Multi-Key Homomorphic Signature for Single Dataset 95

$4.5 .3 \quad$ From a Single Dataset to Multiple Datasets . . . . . . . . 105

4.5.4 Generic Transformations from Weak to Adaptive Security. 110

4.6 Our Multi-Key Homomorphic MAC from OWFs . . . . . . . . . . 119

4.6 .1 Polynomials Representations . . . . . . . . . . . . . . 120

4.6 .2 Efficiency and Succinctness . . . . . . . . . . . . . . . 121

4.6 .3 Correctness $\ldots \ldots \ldots \ldots$. . . . . . . . . . . . . . . . . . . . . . 122

5 Extended Fair Exchange Over the Bitcoin Blockchain: Zero Knowledge Contingent Payments for Services 130

5.1 An Overview of Our Contribution . . . . . . . . . . . . . . . . . . 130 
5.1 .1 Related work . . . . . . . . . . . . . . . . . 131

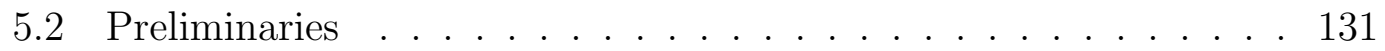

5.2 .1 Bitcoin and Ethereum . . . . . . . . . . . . . . . . 131

5.2 .2 Claw free function pairs . . . . . . . . . . . . . . . 133

$5.2 .3 \quad$ Fair Exchange . . . . . . . . . . . . . . . . . . . . . . . . 134

5.2 .4 Smart Contracts: Fair Exchange over Blockchains . . . . . 135

5.2.5 Zero-Knowledge Contingent Payments: Fair Exchange over Bitcoin . . . . . . . . . . . . . 136

$5.2 .6 \quad$ Example: Pay for Sudoku Solutions . . . . . . . . . . . . . 137

5.3 Attacks on ZKCP with untrusted CRS . . . . . . . . . . . . . 138

5.3.1 ZK-SNARKs from Quadratic Arithmetic Programs . . . . 138

5.3 .2 Learning Information by modifying the CRS . . . . . . . . 141

5.3 .3 Countermeasures . . . . . . . . . . . . . . . . 144

5.4 Contingent Service Payments . . . . . . . . . . . . . . . . 145

5.4.1 Defining ZKCP for Services (ZKCSP) . . . . . . . . . . . . 146

$5.4 .2 \quad$ A ZKCSP Protocol . . . . . . . . . . . . . . . . . . . . . 147

5.4 .3 An alternative ZKCP construction . . . . . . . . . . . . . 150

5.4 .4 A Protocol with private verification . . . . . . . . . . . 150

5.5 Implementation . . . . . . . . . . . . . . . . . . . . . . 151

5.5 .1 Pay-to-Sudoku . . . . . . . . . . . . . . . . 152

5.5.2 Proofs of Retrievability (PoR) over Bitcoin . . . . . . . . . 152

5.5.3 A More Efficient SHA256 Circuit Implementation. . . . . . 154

$\begin{array}{lll}6 & \text { Conclusions } & 156\end{array}$

\begin{tabular}{lr}
\hline Bibliography & 159
\end{tabular} 


\section{List of Publications}

This thesis comprises five papers, the first four have been published to peerreviewed academic conferences and journals. The last one is currently under submission. The following list summarizes the aforementioned publications:

- Dario Catalano, Dario Fiore, and Luca Nizzardo Programmable Hash Functions go Private: Constructions and Applications to (Homomorphic) Signatures with Shorter Public Keys In: Gennaro, R., Robshaw, M. (eds.) CRYPTO 2015. LNCS, vol. 9216, pp. 254-274. Springer, Heidelberg (2015).

- Dario Fiore, Aikaterini Mitrokotsa, Luca Nizzardo and Elena Pagnin Multi-Key Homomorphic Authenticators

In: Cheon, J.H., Takagi, T. (eds.) ASIACRYPT 2016. LNCS, vol. 10032, pp. 499-530. Springer, Heidelberg (2016)

- Matteo Campanelli, Rosario Gennaro, Steven Goldfeder and Luca Nizzardo Zero-Knowledge Contingent Payments Revisited: Attacks and Payments for Services

In: Thuraisingham, B.M., Evans, D., Malkin, T., Xu, D. (eds.) ACM CCS 17, pp. 229-243. ACM Press, October/November 2017.

- Dario Catalano, Dario Fiore, and Luca Nizzardo

Homomorphic Signatures with Sublinear Public Keys via Asymmetric Programmable Hash Functions

In: Design Codes and Cryptography, pp.1-50, December 2017:1

1 This article is based on an earlier article which appears in the proceedings of CRYPTO 2015. 
- Dario Catalano, Dario Fiore, and Luca Nizzardo On the Security Notions for Homomorphic Signatures Under Submission. 


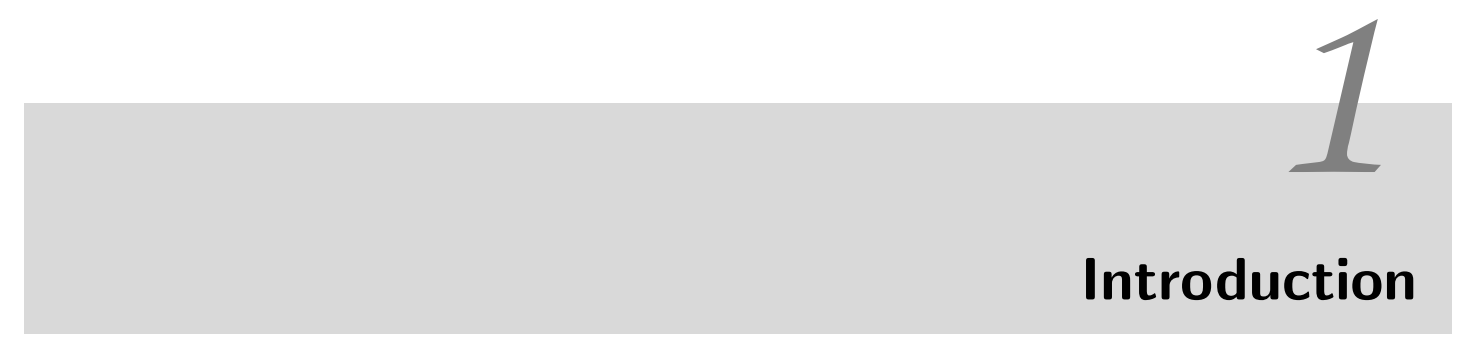

In the last decade, the raise of the Internet has revolutionized our lives: both large companies and individuals have changed the way they access, store, process and consume data. From music to movies, from job market to domotics, from social interaction to financial operations, everything has been largely influenced by the possibilities offered by what we can call the "digital revolution" of Internet.

In particular, one of the most promising technologies that arose in the last years is cloud computing: using a cloud service, anyone (a Client) can outsource data to a remote server (the Cloud), and later on ask the Cloud to perform operations on them. This new paradigm allows for huge potential savings in terms of time, computing power and other kind of infrastructural costs: indeed, it is not necessary to maintain and update a proprietary technological infrastructure anymore, since it is possible to access to pay-per-use services upon request. It is clear that a computing paradigm like this is very attractive but, at the same time, it is evident that one may be concerned about security. Indeed, trusting the Cloud can be problematic for various reasons: more specifically, two main security concerns to be addressed are those about the privacy and authenticity of the data stored and processed in untrusted environments. Intuitively, with privacy we mean that the Client does not want the server to learn any information from the outsourced data, while with authenticity we refer to the need for the Client to verify that the Cloud behaved honestly when asked for any computation over outsourced data.

It has been shown that in such a setting privacy can be solved using cryptographic primitives as, for instance, homomorphic encryption [71]. In this thesis we focus on the orthogonal problem of providing authenticity of data during computation. Towards this goal, our contribution is on advancing the study of homomorphic authenticators.

Another remarkable aspect of the Internet revolution is without any doubt the invention of Bitcoin [100], the first example of decentralized digital currency, which allows users to transfer digital money without relying on any trusted au- 
thority. Other than being appealing for its potential in the framework of payments, Bitcoin became also famous for its underlying technology, the Blockchain. One can think of a Blockchain as a distributed ledger over which all the users can reach consensus, and where all the transactions are recorded in an immutable way. This peculiar feature made the "Blockchain paradigm" to become one of the most popular and promising concepts in the last few years, attracting huge efforts both in terms of research and business investments. In particular, a lot of attention has been devoted to the possible uses of the Blockchain technology beyond Bitcoin. Specifically, this work is focused on achieving fair exchange by profiting of the Blockchain features, where with fair exchange we mean the possibility for two users to swap digital goods such that neither can cheat the other.

\subsection{Our Contribution}

As mentioned earlier, the main contribution of this thesis is on advancing the study of homomorphic authenticators, and to address the problem of fair exchange using the Blockchain. In this section we provide a high-level overview of our results, while more details are given in the following sections.

In Homomorphic authenticators a Client $\mathcal{C}$ authenticates his data using a secret key and outsources the data along with the authenticators to the Cloud. Later on, the Cloud is asked by a third entity (the Verifier) to compute a function $f$ over data previously authenticated by $\mathcal{C}$. Using an evaluation key, the Cloud can derive an authenticator for the output of the function using the authenticators of its inputs; the Verifier receives a message along with an authenticator which he can verify as a correct output of $f$ using a verification key. With respect to this topic, our contribution is mainly threefold.

First, we redefined the security notions for homomorphic signatures (which are homomorphic authenticators with public verifiability) obtaining a security model which is easier to deal with compared with the existing one, especially when addressing real life scenarios. Moreover, in order to obtain homomorphic signature schemes that are secure according to our new stronger model, we built generic compilers that allow to transform any homomorphic signature scheme which is secure in a weaker model into one which is secure in our new model.

Second, we solved an open problem regarding the size of the verification key in homomorphic signature schemes: concretely, in the case of homomorphic signatures for linear functions we manage to reduce the dimension of the verification key from linear to sublinear in the size of the authenticated dataset.

Finally, as a third contribution in the area of homomorphic authentication, we investigated and formalized the notion of homomorphic authenticators for functions which take inputs from multiple Clients (namely, authenticated using different secret keys), and we provided concrete constructions both in the case of private and public verification. Note that before our work, homomorphic authen- 
ticators were inherently limited to deal with inputs provided by a single Client (that is, data that have been all authenticated using the same secret key).

Addressing the problem of fair exchange over the Blockchain, we considered the state of the art protocol, called Zero Knowledge Contingent Payments (ZKCP), which allows for swapping digital goods using the Bitcoin Blockchain as a trusted party. In this context, our contribution is mainly twofold: first, we identified a flaw in the ZKCP and we proposed how to efficiently solve the security issue by changing the original protocol. Moreover, we showed that this new protocol supports additional functionalities which were impossible to achieve before.

\subsection{Authenticated Computations in the Cloud}

As we said in the previous paragraph, the problem addressed in the first part of this thesis deals with the authenticity of computations outsourced to the Cloud. With an homomorphic authenticator (HA, for short) we are in a scenario where a Client $\mathcal{C}$ has some data that he wants to outsource to a remote untrusted server $\mathcal{S}$, without keeping a local copy of it. Not only that: later on a Verifier $\mathcal{V}$ asks the server to perform some computations over the outsourced data and wants to be sure that the computation was executed correctly. To this aim, $\mathcal{C}$ wants that a Verifier $\mathcal{V}$ is able to check that the server is not cheating, namely that $\mathcal{S}$ computed a given function correctly on the very same data provided by $\mathcal{C}$. Nevertheless, since neither $\mathcal{C}$ nor $\mathcal{V}$ have direct access to the data, none of them can directly check the computation. One possible solution to this problem consists in $\mathcal{C}$ authenticating each message with a private authentication key and sending to the server a dataset $\Delta$ of pairs $\left(m_{i}, \sigma_{i}\right)$ where each message comes with its digital signature. In this way, if the Verifier wants to compute a function $f$ over some data $m_{1}, \ldots, m_{n}$ that $\mathcal{C}$ previously outsourced, he can ask the server to send all the pairs $\left\{\left(m_{i}, \sigma_{i}\right)\right\}_{i=1}^{n}$, check that all the digital signatures are valid, and compute $f\left(m_{1}, \ldots, m_{n}\right)$ locally by himself.

Nevertheless, this approach is really inefficient both in terms of bandwidth and storage power, since for each computation everything has to be dowloaded and stored. Not only that: when dealing with devices with reduced memory capacity and limited data connection, like smartphones, it could be simply infeasible (or not profitable) to run a protocol like the one described above.

Homomorphic authenticators provide an efficient and secure solution to this problem.

Using an homomorphic authenticator, the Client authenticates a collection of data items $m_{1}, \ldots, m_{t}$ using her secret key, and send the signed data to an untrusted server. The server can execute a program $\mathcal{P}$ on the signed data and use a public evaluation key to generate a value $\sigma_{\mathcal{P}, y}$ vouching for the correctness of $y=\mathcal{P}\left(m_{1}, \ldots, m_{t}\right)$. Finally, a Verifier $\mathcal{V}$ who is given the tuple $\left(\mathcal{P}, y, \sigma_{\mathcal{P}, y}\right)$ and the 
Client's verification key can use the authenticator to verify the authenticity of $y$ as output of the program $\mathcal{P}$ executed on data authenticated by $\mathcal{C}$. In other words, the Verifier $\mathcal{V}$ can check that the server $\mathcal{S}$ did not tamper with the computation's result.

One of the attractive features of homomorphic authenticators is that $\sigma_{\mathcal{P}, y}$ is succinct, i.e., much shorter than $\mathcal{P}$ 's input size. This means that the server $\mathcal{S}$ can execute a program on a huge amount of data and convince the Verifier $\mathcal{V}$ of its correctness by sending him only a short piece of information. In this framework, there are two possible scenarios: on one hand (and for most of the time) we will assume that the Verifier can use a public verification key which is of course different from the secret one used by the Client to authenticate his messages. This means that the verification of the Server's computation is public: in this case we will refer to homomorphic signatures. On the other hand, we will also consider scenarios where the Client's secret key is needed to verify the Server's computations (in particular, verification is private, then the Verifier and the Client have to share a secret key and must trust each other): in this case we will refer to homomorphic message authentication codes (MACs for short).

The notion of homomorphic signature was (informally) suggested by Desmedt [56] and later more formally introduced by Johnson et al. [85]. The special case of homomorphic signatures for linear functions (called linearly homomorphic signatures) was first considered by Boneh et al. [30] as a key tool to prevent pollution attacks in network coding routing mechanisms. Following this work, several papers further studied this primitive both in the random oracle [69, 32, 31, 45], and in the standard model [12, 47, 13, 48, 63, 14, 46]. In the private verification setting realizations of linearly homomorphic MACs have been proposed by Agrawal et al. in [3].

Several recent works also considered the question of constructing homomorphic authenticators (i.e., signatures and/or MACs) supporting more expressive functionalities. Boneh and Freeman in [31] proposed an homomorphic signature scheme for constant degree polynomials, in the random oracle model. Gennaro and Wichs [70] presented a construction of fully homomorphic MACs based on fully homomorphic encryption in a restricted adversarial model where no verification queries are allowed. Catalano and Fiore [43] proposed a much more efficient homomorphic MAC solution that, while capturing a less expressive class of functionalities (i.e. arithmetic circuits of polynomially bounded degree), allows for verification queries. This latter result was further generalized in [44]. All these constructions of homomorphic MACs achieve adaptive security.

In the public verification setting, Catalano, Fiore and Warinschi 49] proposed a homomorphic signature that achieves adaptive security in the standard model, works for constant degree polynomials and is based on multilinear maps. Moreover, Gorbunov, Vaikuntanathan and Wichs [77] recently proposed the first homomorphic signature construction that can handle boolean circuits of bounded polynomial depth; their scheme is secure in the semi-adaptive model, and is based 
on standard lattices.

\subsubsection{New Security Notions for Homomorphic Signatures}

As a first contribution, this thesis focuses on the security model behind homomorphic signatures, and in particular on its security definitions. The starting point has been the known fact for which defining security for homomorphic signatures is a bit complicated. Indeed, if we consider the usual unforgeability requirements for digital signatures [76], where an adversary who can obtain signatures over messages of its choice can not provide a valid signature for a "new" message, it is clear that these requirements can not be satisfied by homomorphic signatures. The reason behind this relies on the fact that in an homomorphic signature scheme the untrusted remote server must be able to provide a valid signature for an output message, which is of course different from the ones he was given by the Client at the beginning of the protocol. Said in another way, it might seem paradoxical to require unforgeability from a primitive where malleability is a feature (and actually one of the main features) rather than an issue. Intuitively, what we want to say in terms of security is that the only way the server can create a valid signature for any of its outputs is to honestly derive that signature from the signatures over the inputs, depending on the computation $f$ that the Client asked her to compute.

In this sense, the first satisfactory definition was proposed by Boneh and Freeman in [31]. Informally, they solved the paradox above by defining unforgeability with respect to programs (rather than to messages). At an intuitive level this new unforgeability game can be explained as follows. During the training phase the adversary $\mathcal{A}$ is allowed to see the signatures of messages belonging to different datasets. In particular, she can see the signatures corresponding to all the messages corresponding to polynomially many (adaptively chosen) different datasets. The adversary then wins the game if she can produce either a signature on a message $m$ belonging to some previously unseen dataset (type $I$ forgeries) or if, for some previously seen dataset $\Delta=\left\{m_{1}, \ldots, m_{n}\right\}$, she manages to produce a triplet $(\mathcal{P}, \sigma, m)$, such that $\sigma$ verifies correctly but $m \neq \mathcal{P}\left(m_{1}, \ldots, m_{n}\right)$ (type $I I$ forgeries). Thus, for homomorphic signatures security means that no adversary can cheat when claiming correct the output of the given program $\mathcal{P}$.

An important caveat of the Boneh and Freeman [31] definition is that, in order for their security proof to go through, it imposes some restrictions on the queries posed by the adversary. In particular, $\mathcal{A}$ can query (adaptively) signatures on several datasets, but she is required to submit all the messages belonging to each queried dataset. In other words, the definition assumes that for each queried dataset $\Delta$ of size $n, \mathcal{A}$ asks exactly $n$ signing queries ${ }^{n}$. Because of these limitations, in our work we refer to this notion as semi-adaptive security.

\footnotetext{
${ }^{1}$ We remark that the original Boneh-Freeman definition imposes the even stronger restriction that these $n$ messages are queried all at once.
} 
To overcome these restrictions Freeman [63] later proposed a new, more natural, notion where the adversary is allowed to adaptively query messages one by one and even to sprinkle queries from different datasets. Because of its increased adaptativity, we call this notion standard adaptive security or simply adaptive security.

Adaptive security, while very natural, has a dark side. Indeed, loosening the query-all requirement implies that the adversary could provide a forgery $(\mathcal{P}, \sigma, m)$ that corresponds to a previously seen dataset $\Delta$, but for which $\mathcal{A}$ did not ask sufficiently many queries for $\mathcal{P}$ to be well defined with respect to $\Delta$. For instance, $\mathcal{A}$ could provide a forgery that depends on "unqueried" elements of the dataset, in which case the output of the program might not even be specified. Freeman called these forgeries, where verification succeeds but $\mathcal{P}$ is not well defined, type III forgeries.

Type III forgeries are nasty animals as, while it is relatively easy to deal with them in cases where $\mathcal{P}$ is a linear [63] or a low degree polynomial [43, [4], in general it might be very hard (or even impossible) for the challenger to establish well-definedness for general programs $\mathcal{P}$ (i.e. determine if $\mathcal{P}$ is well defined with respect to $\Delta$ ). The consequence of this is that, to prove adaptive security, one either has to restrict to low degree polynomials or to impose other kind of restrictions to the computation ${ }^{2}$. This state of affairs is quite annoying as having expressive, yet easy to use definitions, is a fundamental step towards a better understanding of cryptographic primitives. What we did was to put forward a new notion of security for homomorphic signatures that is both stronger and much simpler to use than the standard one discussed above. Our notion, that we call strong adaptive security, extends semi-adaptive security by encompassing a very simple and natural notion of type III forgeries. Informally, these still corresponds to forgeries containing some "unqueried" element of the dataset. The simplification comes from the fact that our new definition does not need to rely on well-defined programs anymore. As on of the main contributions, we showed that strong adaptive security is surprisingly easy to achieve. Specifically, we propose a compiler that, starting from an homomorphic signature $\Sigma$ providing semi-adaptive security, converts it into a, similarly expressive, scheme achieving strong adaptive security. The compiler uses, as building block, a semi-adaptive secure signature scheme $\Sigma_{\mathrm{OR}} \equiv\left(\Sigma_{\mathrm{OR}} \cdot\right.$ KeyGen, $\Sigma_{\mathrm{OR}} \cdot$ Sign, $\Sigma_{\mathrm{OR}}$. Eval, $\Sigma_{\mathrm{OR}} \cdot$ Ver $)$ that supports OR operations over $\mathbb{Z}_{2}$.

Clearly, if $\Sigma$ supports arbitrary boolean circuits, then $\Sigma_{\text {OR }}$ can be instantiated using $\Sigma$. In such a case our result can be seen as way to "bootstrap" semiadaptive security to strong adaptive security. If, on the other hand, $\Sigma_{\text {OR }}$ cannot be instantiated using $\Sigma$, our result still provides a way to get strong adaptive

\footnotetext{
${ }^{2}$ An example is the solution proposed by Gennaro and Wichs in [70. There the authors suggest to use hash trees to be able to (implicitly) deal with type III forgeries. Their specific realization, however, is specific to the symmetric setting as relies on pseudorandom functions and does not allow for public verifiability.
} 
security, under the additional assumption that OR homomorphic signatures exist. A detailed description of this result is actually the object of Chapter 2 .

\subsubsection{Shorter Public Keys via Asymmetric Programmable Hash Functions}

As a second contribution, we worked on the verification key size for homomorphic signatures. Despite the significant research work in the area, before our work all the existing homomorphic signature schemes that are proven secure in the standard model suffered from a verification key that is at least linear in the size $|\Delta|$ of the signed datasets.

It is true that the cost of storing such large verification key can be, in principle, amortized since the key can be re-used for multiple datasets; nevertheless, this limitation represented a challenging open question from both a theoretical and a practical point of view. From a theoretical point of view, considered the stateof-the-art before our work, it seemed unclear whether achieving an homomorphic signature scheme in the standard-model scheme with a key of length $o(|\Delta|)$ was possible at all. From a practical perspective, a linear public key might be simply unaffordable by a user Bob who has limited storage capacity.

We solved the above open problem by profiting by the features of a new tool we introduced: Asymmetric Programmable Hash Functions (APHFs for short), which can replace Random Oracles in different cryptographic construction, including linearly homomorphic signatures.

Concretely, we obtain the first linearly homomorphic signature scheme, secure in the standard model, achieving a public key which is sub-linear in both the dataset size and the dimension of the signed vectors. As an additional result, we obtain regular signature schemes, matching the efficiency of the ones in [116], thus providing the shortest signatures in the standard model with a public key shorter than in [82]. This contribution is the object of Chapter 3.

\subsubsection{Homomorphic Authenticators Dealing With Multi- ple Keys}

As a third contribution, we addressed the problem of multi-key homomorphic authenticators (both signatures and MACs): up to now, the notion of homomorphic authenticators had inherently been single-key, i.e., homomorphic computations are allowed only on data authenticated using the same secret key. This characteristic is obviously a limitation and prevents homomorphic authenticators schemes from suiting scenarios where the data is provided (and authenticated) by multiple users.

Consider the example of healthcare institutions which need to compute on data collected by several hospitals or even some remote-monitored patients. Similarly, it is often required to compute statistics for time-series data collected from 
multiple users e.g., to monitor usage data in smart metering, clinical research or to monitor the safety of buildings. Another application scenario is in distributed networks of sensors. Imagine for instance a network of sensors where each sensor is in charge of collecting data about air pollution in a certain area of a city, it sends its data to a Cloud server, and then a central control unit asks the Cloud to compute on the data collected by the sensors (e.g., to obtain the average value of air pollution in a large area).

A trivial solution to address the problem of computing on data authenticated by multiple users is to use homomorphic authenticators in such a way that all data providers share the same secret authentication key. The desired functionality is obviously achieved since data would be authenticated using a single secret key. This approach however has several drawbacks. The first one is that users need to coordinate in order to agree on such a key. The second one is that in such a setting there would be no technical/legal way to differentiate between users (e.g., to make each user accountable for his/her duties) as any user can impersonate all the other ones. The third and more relevant reason is that sharing the same key exposes the overall system to way higher risks of attacks and makes disaster recovery more difficult: if a single user is compromised the whole system is compromised too, and everything has to be reinitialized from scratch.

In contrast, we provided an innovative solution through the notion of multi-key homomorphic signatures. This primitive guarantees that the corruption of one user affects the data of that user only, but does not endanger the authenticity of computations among the other (un-corrupted) users of the system. Moreover, the proposed system is dynamic, in the sense that compromised users can be assigned new keys and be easily reintegrated.

Our contribution here consists in elaborating a suitable definition of multi-key homomorphic signatures and in proposing the first construction of a multi-key homomorphic signature scheme, as can be seen in Chapter 4 .

\subsection{Fair Exchange Over The Bitcoin Blockchain}

The second part of this work is related to the problem of fair exchange: imagine that two parties want to swap digital goods such that neither can cheat the other. This problem has been studied for decades, and indeed it has been shown that fairness is unachievable without the aid of a trusted third party [53]. However, using Bitcoin or other Blockchain-based cryptocurrencies, it has been demonstrated that fair-exchange can be achieved in a completely trustless manner. The previous results were not incorrect: a third party is definitely necessary, but the key innovation that Bitcoin brings to fair exchange is that the Blockchain can fill the role of the trusted party, and essentially eliminate trust.

Consider Alice, an avid fan of brainteasers that has a Sudoku puzzle that she is stumped on. After trying for days to solve the puzzle, Alice gives up and 
posts the puzzle on an online message board proclaiming, "I will pay whoever provides me the solution to this puzzle". Bob sees this message, solves the puzzle, and wants to sell Alice the solution. But there's a problem: Alice wants Bob to first provide the solution so that she can verify it's correct before she pays him, whereas Bob insists that he will not send Alice the solution until he has been paid. This is the classical problem of fair exchange: neither party wants to impart with its good before being sure that it will receive the other good in exchange.

To solve this problem, Alice and Bob could use a Blockchain: nevertheless, if one want to implement such a protocol over the Bitcoin Blockchain, the fact that its scripting language is really limited is a key point that has to be taken into account. A solution to this problem was provided in 2011 and it is known as Zero Knowledge Contingent Payments (ZKCP for short): as a fourth contribution of this work, we found a flaw in the original ZKCP protocol, we fixed it and we also proposed a novel approach to the problem which allows for extending the functionalities of the original protocol. Concretely, if before one could only implement fair exchange over the Bitcoin Blockchain to swap digital goods, with our new protocol called Zero Knowledge Contingent Payments for Services (ZKCSP for short) it is now possible to implement fair exchange for verifying the delivery of services: as an example, this is quite remarkable in a situation where one wants to pay for a remote storage service and wants to periodically verify that the remote server is actually keeping storing his data.

\subsubsection{Zero Knowledge Contingent Payments}

As we said, Bitcoin's scripting language is limited, and does not allow one to directly specify arbitrary programs or conditions that are necessary to spend money. Zero Knowledge Contingent Payment (ZKCP) protocols [36, 25, 94 to allow fair exchange over the Bitcoin Blockchain. The protocol makes use of a feature of the Bitcoin scripting language that allows one to create a payment transaction that specifies a value $y$ and allows anyone who can provide a preimage $k$ such that $\operatorname{SHA256}(k)=y$ to claim the bitcoins ${ }^{3}$.

In the ZKCP protocol, Bob knows a solution $s$ and encrypts the solution to the puzzle using a key $k$ such that $\operatorname{Enc}_{k}(s)=c$. Bob also computes $y$ such that SHA256 $(k)=y$. He then sends Alice $c$ and $y$ together with a zero-knowledge proof that $c$ is an encryption of $s$ under the key $k$ and that $\operatorname{SHA256}(k)=y$. Once Alice has verified the proof, she creates a transaction to the Blockchain that pays Bob $n$ bitcoins, and specifies that Bob can only claim the funds if he provides a value $k^{\prime}$ such that SHA256 $\left(k^{\prime}\right)=y$. Bob then published $k$ and claims the funds. Alice, having learned $k$ can now decrypt $c$, and hence she learns $s$.

When ZKCP was first introduced in 2011 it was only theoretical as there was no known efficient general purpose zero-knowledge protocol that could be used

\footnotetext{
${ }^{3}$ We are simplifying the protocol here. See Chapter 5 for full details.
} 
for the necessary proofs. Since then, however, advances have been made in this area, and there are now general-purpose Succinct Non-Interactive Arguments of Knowledge (ZK-SNARK) protocols that allow for the practical implementation of the necessary proofs. The protocol was refined to use SNARKs, and a sampleimplementation for the Sudoku problem was also made available [36].

All NIZK proofs require a trusted party to generate the common reference string (CRS) for the production and the verification of the proof. The introduction of a third party, however, even to generate the parameters, is undesirable recall that the entire point of ZKCP is to solve the fair exchange protocol in a completely trustless manner.

To eliminate the need for a trusted third party, proofs in ZKCP are made to convince one person - the buyer. It was natural therefore, for the buyer to serve as the trusted third party. Since the buyer trusts herself, she will be convinced of the correctness of the proofs. Using this observation, the ZKCP protocol specifies that the buyer should generate the CRS, and indeed the Sudoku implementation follows these guidelines.

But in ZKCP, there are two potential adversaries: the seller and the buyer. A malicious seller would try to cheat by producing a false proof that convinces the buyer to send her money even though she will not receive the solution. Indeed, the current protocol protects against this attack. Since the buyer generates the CRS, the seller (prover) is unable to produce an incorrect proof that will be accepted by the buyer (Verifier).

But the buyer can also be malicious. Indeed, if the buyer is able to break the zero-knowledge property of the proof, she may learn part of the solution from the seller even without paying! Intuitively, the buyer can modify the CRS such that the proof that the seller provides actually leaks some bits of the solution.

In the original SNARKs paper, it was assumed that the CRS was generated honestly, and indeed the proof of the zero-knowledge property made use of this fact [68]. When this assumption is violated, a malicious party can craft a CRS that allows it to break the zero-knowledge property and learn information about the witness. We note that if the Prover checks that the CRS is "well formed", the SNARK in [68] remains Witness Indistinguishable (WI) - however this is not sufficient since in this case the witness is the Sudoku solution which is unique (and therefore even if the protocol is WI, information can be learned about the witness). We also note that with some additional more expensive checks, the SNARK in 68, remains ZK [17, 65, 1]. In ZKCP however neither of these checks are performed, and therefore, a malicious buyer can generate a malicious CRS that allows it to learn information from the seller's proof without paying. We show an attack on the "pay to Sudoku" protocol that proceeds along these lines, and we also provide code [41, 42] that implements the attack and shows how one can break the zero-knowledge property and learn information in the sample sudoku code [36]. 


\subsubsection{Zero Knowledge Contingent Payments for Services: Fixing and Extending ZKCP}

While issues arise when the Verifier generates the CRS, the ZKCP high-level idea remains elegant and appealing. Therefore in Chapter 5 we discuss several ways to construct ZKCPs which do not require the help of a trusted party.

One way is to require that the CRS is constructed via a two-party secure computation protocol jointly by buyer and seller, a solution which allows them to "recycle" the CRS over several ZKCP executions. A similar approach was adopted by the designers of Zcash [105].

Another way is to use the notion of Subversion-NIZK [17], where ZK is preserved even when the Verifier chooses the CRS. As we pointed out above, this requires the Prover to perform some "well formedness" checks on the CRS, which however can be somewhat expensive (as opposed to the minimal checks described in [68] to guarantee witness-indistinguishability).

At the end the simplest solution was to rely on a different type of protocol for Zero-Knowledge Contingent Service Payments (ZKCSP)

What we did was to extend the idea of ZKCP to a new class of problems: paying for digital services.

Consider Alice, a user of a subscription online file storage service, FileBox. FileBox offers a service that for a small fee, it will provide a succinct proof-ofretrievability (PoR) [107] to its users demonstrating that all of that user's files are being stored. Alice would like to pay for this service, and thus we have a far exchange problem: Alice wants to pay once she receives proof that the files are being stored, whereas FileBox will only send the proof once it has been paid.

Notice that unlike the Sudoku example, Alice does not want any digital good (i.e. she doesn't want them to send her all the files). Instead, she just wants Filebox to demonstrate that they are indeed still storing the files.

The ZKCP protocol will fail in this case. If we try to apply this protocol and view the PoR as a "good" that Alice wants to receive, then the first step of a ZKCP protocol is to have FileBox create a proof that it has a PoR and send the encrypted PoR to Alice.

But a proof of a PoR is itself a PoR, and thus once Alice receives this zeroknowledge proof, she can abort the protocol as she already received the proof that she desired without paying.

As a second motivation, consider an online Bitcoin exchange that will provide proofs of solvency as a service for a fee. Often exchanges do not want to leak their inner details, and thus they may use Provisions [54], a privacy-preserving proof of solvency that shows that they are solvent without leaking their private accounting details. Bob stores his coins with this exchange and wishes to pay for the proof, and thus a fair exchange situation arrives.

Again, if we try to apply a ZKCP protocol, it will fail. If the exchange gives a zero knowledge proof of a proof of solvency, that itself is a proof of solvency, 
and Bob has received what he wants and does not need to pay.

To address this issue, we introduced Zero-Knowledge Contingent Service Payments (ZKCSP). To illustrate, let's focus on the PoR example. Let $v$ be the verification algorithm for the PoR. What Alice wants then is for FileBox to demonstrate that it knows $m$ such that $v(m)=1$.

Intuitively, our ZKCSP protocol works as follows: The prover outputs a string $y$ and gives a zero-knowledge proof that attests to the following:

If $v(m)=1$, then I know the preimage of $y$ under SHA256. But, if $v(m)=0$, then the probability that I know a SHA256 preimage of $y$ is negligible.

We only provide the intuition here, but in Section 5.4 we show how we can efficiently construct proofs of this form. There we also prove that it is sufficient for the underlying SNARK to be Witness-Indistinguishable, and therefore the security of the protocol can be achieved even if the Verifier chooses the CRS, provided that the Prover performs the minimal checks required to guarantee witness indistinguishability.

\subsection{Thesis Organization}

Chapter 2, 3 and 4 collect our results which regard the paradigm of Cloud computing, and, more specifically, homomorphic authenticators. In details:

In Chapter 2 we present our new security model for homomorphic signatures.

Chapter 3 presents our results in terms of efficiency of homomorphic signatures, that is how it is possible to obtain a linearly homomorphic signature scheme with verification key size sublinear with respect to the dataset size.

In Chapter 4 we discuss our contribution with respect to the functionalities of homomorphic authenticators: we introduce multi-key homomorphic authenticators and we present the constructions of both a multi-key homomorphic signature scheme and of a multi-key homomorphic message authentication codes.

Chapter 5 collects our results in the framework of E-commerce and, more specifically, fair exchange over the Bitcoin blockchain.

After Chapter 5 we present our conclusions and future research directions. 


\section{Improving Definitions: A New Framework for Homomorphic Signatures}

In this Chapter we present our new security model for homomorphic signatures. To this aim, after presenting an overview of our contribution, we will start by a formal description of this cryptographic primitive. We will also present the details of the existing security model, analyzing their strengths and weaknesses, before getting into the technical details of both our new definition and compilers.

\subsection{An Overview of Our Contribution}

As we presented in the introduction, properly defining security for homomorphic signatures is tricky, and the state of the art of security notions for homomorphic signatures seems quite unsatisfactory. Having expressive, yet easy to use, definitions is indeed a fundamental step towards a better understanding of cryptographic primitives.

A Stronger and Simpler Security Notion. To address the issues of adaptive security, we consider a new security notion that is both simpler and stronger than the one in [63]. This notion, that we call strong adaptive security, is the public key version of the one proposed by Gennaro and Wichs [70] for homomorphic message authenticators (the secret key equivalent of homomorphic signatures). 1 Strong adaptive security deals with the case of programs with missing inputs in a simple way: if the triplet $(\mathcal{P}, m, \sigma)$ returned by the adversary verifies correctly and some inputs of $\mathcal{P}$ were not queried during the experiment, then it is considered a forgery (we call it a Type 3 Strong forgery).

Compared to previous notions, strong adaptive security has several advantages. First, the winning condition of the experiment is efficiently computable, thus avoiding the issues that may arise when proving and using homomorphic

\footnotetext{
${ }^{1}$ With some adaptations to deal with multiple datasets which was not considered in [70].
} 
signatures. Second, the new forgery definition is arguably much simpler to state and work with. Finally, being a strengthening of adaptive security, homomorphic signature schemes that are strongly adaptive secure can be used in more application scenarios as discussed before.

Realizing Strong Adaptive Security, Generically. If we aim for strong adaptive security to be the "right" strong notion to use for homomorphic signatures, then we face the problem that virtually all existing schemes are not secure under this strong notion. This is the case for those schemes that support linear or low-degree polynomials and were proven secure under the adaptive notion of [63, as well as for the recently proposed leveled homomorphic scheme for circuits [77] which is only semi-adaptive secure. Notably, all these constructions break down in the new security experiment as they do not tolerate adversaries that issue Type 3 Strong forgeries. The only scheme which stands security in this stronger model is a recent proposal of Elkhiyaoui et al. [59] which supports constant-degree polynomials and relies on multilinear maps in the random oracle model.

To remedy this situation, our main contribution is to show that strong adaptive security can be easily achieved without additional assumptions and in the standard model. Specifically, our main result is a generic compiler that, starting from an homomorphic signature scheme $\Sigma$ satisfying semi-adaptive security, converts $\Sigma$ into a strongly adaptive secure scheme that supports the same class of functions.

The compiler uses, as additional building block, a semi-adaptive secure signature scheme $\Sigma_{\mathrm{OR}}$ that supports OR operations over $\mathbb{Z}_{2}$. Clearly, if $\Sigma$ supports arbitrary boolean circuits, then $\Sigma_{\mathrm{OR}}$ can be instantiated using $\Sigma$ itself. In such a case, our result is thus providing a transformation that "bootstraps" semi-adaptive security to strong adaptive security. If, on the other hand, $\Sigma_{\mathrm{OR}}$ cannot be instantiated using $\Sigma$, our result still provides a way to get strong adaptive security, under the additional assumption that semi-adaptive secure OR-homomorphic signatures exist. Nevertheless, since very few concrete examples of OR-homomorphic signatures are known (essentially, only one [77]), even if we think that this is not a limitation by itself, we asked whether a similar result could be obtained out of some more widely studied primitive. Along this direction, our second result is another compiler that combines a semi-adaptive secure scheme $\Sigma$ together with a semi-adaptive secure linearly-homomophic signature $\Sigma_{\mathrm{LH}}$ that works for messages over a large ring, say $\mathbb{Z}_{p}$. This combination yields a homomorphic signature scheme that is strongly adaptive secure and supports the same class of functions supported by $\Sigma$. A limitation of this second transformation is that it applies only to schemes that are leveled homomorphic (i.e., for circuits of bounded depth). As an interesting feature, however, this result shows that strong adaptive security can be obtained from linearly-homomorphic schemes, a class of constructions for which many constructions are known (most of which are also way more efficient 
in practice than [77]).

Both our transformations hold in the standard model, and they preserve three properties of homomorphic signatures: composability, context-hiding and efficient-verification (so, security can be upgraded without penalties). Context hiding deals with privacy and informally says that signatures on computation's outputs do not reveal information on the inputs. The latter instead fully enables the use of homomorphic signatures for verifiable delegation of computation, by requiring that verifying a signature for a program $\mathcal{P}$ is asymptotically faster (in an amortized, offline-online sense) than recomputing $\mathcal{P}$ from scratch.

We point out that our compilers are completely generic with respect to the semi adaptive secure scheme. This means, for instance, that when applied to the recent (leveled) fully homomorphic solution of [77] they lead to homomorphic signature schemes for general circuits achieving strong adaptive security.

On the Importance of Strong Adaptive Security. As an important application of (strong) adaptive secure homomorphic signatures, we mention certified computation on streaming data. Consider a scenario where a user Alice outsources a stream of data $m_{1}, m_{2}, \ldots$ to an untrusted Cloud, so that the Cloud can compute a program $\mathcal{P}$ on the current snapshot $\left(m_{1}, \ldots, m_{i}\right)$ and post the result publicly (e.g., on a third party website). Using homomorphic signatures, Alice can sign each element of the data stream, while the Cloud can compute a homomorphic signature $\sigma_{\mathcal{P}, y_{i}}$ on every computed result $y_{i}=\mathcal{P}\left(m_{1}, \ldots, m_{i}\right)$ and post $\left(y_{i}, \sigma_{\mathcal{P}, y_{i}}\right)$. This way, anyone with the only knowledge of Alice's public key is able to check the results validity. Notably, the Cloud can produce the certified results in a completely non-interactive fashion, and no communication between Alice and the verifiers is needed (except, of course, for sending the public key). In such a scenario, where datasets grow dynamically and one performs computations on their current version, (strong) adaptive security is fundamental as it prevents the cloud from claiming to have results computed on dataset elements that it did not receive (yet). This is particularly relevant in scenarios where there is no communication between the signer and the verifiers, who may not be aware of the current status of the outsourced stream. Furthermore, strong adaptive security is important in the case of very large, potentially unbounded, datasets (as in the streaming case) as one cannot assume that the adversary queries the whole dataset. This actually shows an inherent limitation of semi-adaptive security, which cannot cope with datasets of arbitrarily large, unbounded, size. Indeed, to fit the requirements of the definition, adversaries would be required to ask signing queries on the whole dataset. However, if datasets are unbounded either the notion of whole dataset does not exist, or it can be approximated by configuring the scheme to work on a dataset of exponential size, which could not be queried in full by a polynomially bounded adversary.

As a final note, we remark that, in settings where the messages $m_{1}, \ldots, m_{i}$ are signed sequentially, one at a time, it might be tempting to address the limita- 
tions of semi-adaptive security via (standard) signatures as follows. One simply includes a signature of the largest index $i$ signed so far. When verifying an homomorphic signature for $\mathcal{P}\left(m_{1}, \ldots, m_{k}\right)$, where $k$ is the largest index touched by $\mathcal{P}$, one also requires a (standard) signature on $k$. This solution has the drawback of requiring an ordering of indexes. More seriously, it only works in contexts where messages are signed in index-increasing order. Our solution, on the other hand, encompasses the more general case where messages are signed in completely arbitrary order (and without imposing additional constraints on the underlying indexes).

\subsection{Related work}

As we pointed out in Section 1.2, homomorphic signatures are a cryptographic tool that received increasing attention in the cryptographic community in the last few years: we point out that all the existing schemes that we listed obtain semiadaptive or adaptive security. In particular, for what regards linearly homomorphic signature schemes, adaptive security is implied by semi-adaptive security, as shown in [63]. Moreover, in homomorphic signature schemes for polynomials over finite fields, the adaptive security underlying conditions can be efficiently tested in a probabilistic way. Nevertheless, for more general homomorphic signature schemes this notions represent a serious limitation.

We also notice that a work from Chase et al. [50] on malleable signatures considered a problem similar to the one addressed in this work, i.e., elaborating a definition that allows one to establish, in an efficient way, when the signature produced by the adversary is a valid forgery. They deal with this problem by formalizing the idea that the adversary "must know" the function and the input that were used to obtain the forgery. To formalize this idea, their definition asks for the existence of a black-box extractor that must extract this information from what is in the view of the game and the output of the adversary. Unfortunately, this type of definition is impossible to achieve when one considers the case of succinct homomorphic signatures for $n$-ary functions, as we do in our work. The reason is simply that the extractor should extract an amount of information (such as the function input) that is much larger than what is in its input.

\subsection{An Overview of Our Compiler}

To obtain strongly-adaptive secure homomorphic signatures from semi-adaptive secure ones, we propose a compiler that takes a semi-adaptive secure scheme $\Sigma$ and upgrades its security with the help of an additional building block: an homomorphic signature scheme $\Sigma_{\mathrm{OR}}$ that supports OR operations over $\mathbb{Z}_{2}$. The basic idea of our compiler is to use $\Sigma_{\mathrm{OR}}$ to additionally sign a bit ' 0 ' for every dataset input. The homomorphic properties of this scheme then guarantee that 
the resulting bit remains 0 if and only if one properly operates on ' 0 ' bits. This can be achieved either directly, by employing an or-homomorphic scheme, or indirectly, via an homomorphic signature for additions (over a sufficiently large ring $\mathbb{Z}_{p}$ ). This latter construction is more efficient, but it comes with restrictions. Indeed, to avoid false positives (i.e., invalid signatures that are interpreted as correct ones), $p$ should be large, i.e., larger than $c^{d}$, where $c$ is the (constant) fan-in and $d$ the maximum depth of the supported circuits.

More in detail, the compiler works as follows. For every dataset input $m$, in addition to signing $m$ using the scheme $\Sigma$, we also sign the bit ' 0 ' using $\Sigma_{\text {OR }}$. So, every signature now consists of a pair $\left(\sigma_{m}, \sigma_{b}\right)$, where $\sigma_{m}$ is a signature with the scheme $\Sigma$ on some message $m$, and $\sigma_{b}$ is a signature of a bit $b$ with the scheme $\Sigma_{\mathrm{OR}}$. Next, at every gate $g$ we compute $g$ homomorphically on the $\sigma_{m}$ 's components, and we compute OR homomorphically on the $\sigma_{b}$ 's. Finally, signature verification consists into the usual verification of $\sigma_{m}$ (for a program $\mathcal{P}$ ), plus checking that $\sigma_{b}$ verifies for 0 for a computation that is simply an OR of all the dataset input bits. The reason why this makes the scheme resistant to strong Type-3 forgeries is that when the adversary does not ask all the dataset inputs (and thus misses a signature of ' 0 ' on the missing inputs) it is forced to create a forgery for the $\sigma_{b}$ component. The latter must verify for ' 0 ' by construction. However, one of the missing bits could be set to be ' 1 ' (notice indeed that the adversary does not see it), thus making the correct output of the OR computation ' 1 '. Hence the signature returned by the adversary must verify for an incorrect output, i.e., it is a forgery for $\Sigma_{\mathrm{OR}}$.

Notation. We denote with $\lambda \in \mathbb{N}$ a security parameter. A probabilistic polynomial time (PPT) algorithm $\mathcal{A}$ is a randomized algorithm for which there exists a polynomial $p(\cdot)$ such that for every input $x$ the running time of $\mathcal{A}(x)$ is bounded by $p(|x|)$. We say that a function $\epsilon: \mathbb{N} \rightarrow \mathbb{R}^{+}$is negligible if for every positive polynomial $p(\lambda)$ there exists $\lambda_{0} \in \mathbb{N}$ such that for all $\lambda>\lambda_{0}: \epsilon(\lambda)<1 / p(\lambda)$. If $S$ is a set, $x \stackrel{\$}{\leftarrow} S$ denotes the process of selecting $x$ uniformly at random in $S$. If $\mathcal{A}$ is a probabilistic algorithm, $y \stackrel{\$}{\leftarrow} \mathcal{A}(\cdot)$ denotes the process of running $\mathcal{A}$ on some appropriate input and assigning its output to $y$. For a positive integer $n$, we denote by $[n]$ the set $\{1, \ldots, n\}$.

\subsection{Defining Homomorphic Signatures}

In this section we recall the definition of homomorphic signatures. This definition extends the one by Freeman in 63] in order to work with the general notion of labeled programs [70].

Labeled Programs [70]. A labeled program $\mathcal{P}$ is a tuple $\left(f, \tau_{1}, \ldots, \tau_{n}\right)$ such that $f: \mathcal{M}^{n} \rightarrow \mathcal{M}$ is a function of $n$ variables (e.g., a circuit) and $\tau_{i} \in\{0,1\}^{*}$ is a label of the $i$-th input of $f$. Labeled programs can be composed as follows: given $\mathcal{P}_{1}, \ldots, \mathcal{P}_{t}$ and a function $g: \mathcal{M}^{t} \rightarrow \mathcal{M}$, the composed program $\mathcal{P}^{*}$ is the 
one obtained by evaluating $g$ on the outputs of $\mathcal{P}_{1}, \ldots, \mathcal{P}_{t}$, and it is denoted as $\mathcal{P}^{*}=g\left(\mathcal{P}_{1}, \ldots, \mathcal{P}_{t}\right)$. The labeled inputs of $\mathcal{P}^{*}$ are all the distinct labeled inputs of $\mathcal{P}_{1}, \ldots, \mathcal{P}_{t}$ (all the inputs with the same label are grouped together and considered as a unique input of $\left.\mathcal{P}^{*}\right)$.

Let $f_{i d}: \mathcal{M} \rightarrow \mathcal{M}$ be the identity function and $\tau \in\{0,1\}^{*}$ be any label. We refer to $\mathcal{I}_{\tau}=\left(f_{i d}, \tau\right)$ as the identity program with label $\tau$. Note that a program $\mathcal{P}=\left(f, \tau_{1}, \cdots, \tau_{n}\right)$ can be expressed as the composition of $n$ identity programs $\mathcal{P}=f\left(\mathcal{I}_{\tau_{1}}, \cdots, \mathcal{I}_{\tau_{n}}\right)$.

Definition 1 (Homomorphic Signature). A homomorphic signature scheme HomSign consists of a tuple of PPT algorithms (KeyGen, Sign, Ver, Eval) with the following syntax:

$\operatorname{KeyGen}\left(1^{\lambda}, \mathcal{L}\right)$ the key generation algorithm takes as input a security parameter $\lambda$, a description of the label space $\mathcal{L}$ (which fixes the maximum data set size $N)$, and outputs a public key $\mathrm{vk}$ and a secret key sk. The public key $\mathrm{vk}$ contains a description of the message space $\mathcal{M}$ and the set $\mathbb{F}$ of admissible functions.

Sign(sk, $\Delta, \tau, m)$ the signing algorithm takes as input a secret key sk, a data set identifier $\Delta \in\{0,1\}^{*}$, a label $\tau \in \mathcal{L}$, a message $m \in \mathcal{M}$, and it outputs a signature $\sigma$.

$\operatorname{Eval}\left(\mathrm{vk}, f, \sigma_{1}, \ldots, \sigma_{n}\right)$ the evaluation algorithm takes as input a public key $\mathrm{vk}$, a function $f \in \mathbb{F}$ and a tuple of signatures $\left\{\sigma_{i}\right\}_{i=1}^{n}$ (assuming that $f$ takes $n$ inputs). It outputs a new signature $\sigma$.

$\operatorname{Ver}(\mathrm{vk}, \mathcal{P}, \Delta, m, \sigma)$ the verification algorithm takes as input a public key $\mathrm{vk}$, a labeled program $\mathcal{P}=\left(f, \tau_{1}, \ldots, \tau_{n}\right)$ with $f \in \mathbb{F}$, a dataset identifier $\Delta$, a message $m \in \mathcal{M}$, and a signature $\sigma$. It outputs either 0 (reject) or 1 (accept).

A homomorphic signature scheme is required to satisfy the properties of authentication correctness, evaluation correctness and succinctness that we describe below.

Authentication Correctness. Intuitively, a homomorphic signature scheme has authentication correctness if the signature generated by $\operatorname{Sign}(\mathrm{sk}, \Delta, \tau, m)$ verifies correctly for $m$ as the output of the identity program $\mathcal{I}_{\tau}$ on a dataset with identifier $\Delta$. More formally, a scheme HomSign satisfies the authentication correctness property if for a given label space $\mathcal{L}$, all key pairs $($ sk, vk $) \leftarrow \operatorname{KeyGen}\left(1^{\lambda}, \mathcal{L}\right)$, any label $\tau \in \mathcal{L}$, dataset identifier $\Delta \in\{0,1\}^{*}$, and any signature $\sigma \leftarrow \operatorname{Sign}(\mathrm{sk}, \Delta, \tau$, $m), \operatorname{Ver}\left(\mathrm{vk}, \mathcal{I}_{\tau}, \Delta, m, \sigma\right)$ outputs 1 with all but negligible probability.

Evaluation Correctness. Intuitively, this property says that running the evaluation algorithm on signatures $\left(\sigma_{1}, \ldots, \sigma_{t}\right)$ such that each $\sigma_{i}$ verifies for $m_{i}$ 
as the output of a labeled program $\mathcal{P}_{i}$ and a dataset with identifier $\Delta$, produces a signature $\sigma$ which verifies for $g\left(m_{1}, \ldots, m_{t}\right)$ as the output of the composed program $g\left(\mathcal{P}_{1}, \ldots, \mathcal{P}_{t}\right)$ and same dataset $\Delta$. More formally, fix a key pair (vk, sk) $\stackrel{\$}{\leftarrow} \operatorname{KeyGen}\left(1^{\lambda}, \mathcal{L}\right)$, a function $g: \mathcal{M}^{t} \rightarrow \mathcal{M}$, and any set of program $/$ message/signature triples $\left\{\left(\mathcal{P}_{i}, m_{i}, \sigma_{i}\right)\right\}_{i=1}^{t}$ such that $\operatorname{Ver}\left(\mathrm{vk}, \mathcal{P}_{i}, \Delta, m_{i}, \sigma_{i}\right)=1$. If $m^{*}=g\left(m_{1}, \ldots, m_{t}\right), \mathcal{P}^{*}=g\left(\mathcal{P}_{1}, \ldots, \mathcal{P}_{t}\right)$, and $\sigma^{*}=\operatorname{Eval}\left(\mathbf{v k}, g, \sigma_{1}, \ldots, \sigma_{t}\right)$, then $\operatorname{Ver}\left(\mathrm{vk}, \mathcal{P}^{*}, \Delta, m^{*}, \sigma^{*}\right)=1$ holds with all but negligible probability.

Succinctness. A homomorphic signature scheme is said to be succinct if, for a fixed security parameter $\lambda$, the size of signatures depends at most logarithmically on the size of the input dataset. More formally, HomSign satisfies succinctness if there exists a polynomial $p(\lambda)$ such that for all $(\mathrm{vk}, \mathrm{sk}) \stackrel{\$}{\leftarrow} \operatorname{KeyGen}\left(1^{\lambda}, \mathcal{L}\right)$, all $\left(m_{1}, \ldots, m_{t}\right) \in \mathcal{M}^{t}$, all $\left(\tau_{1}, \ldots, \tau_{t}\right) \in \mathcal{L}^{t}$, any $\Delta \in\{0,1\}^{*}$, and all functions $f \in \mathbb{F}$, if $\sigma_{i} \stackrel{\$}{\leftarrow} \operatorname{Sign}\left(\mathrm{sk}, \Delta, \tau_{i}, m_{i}\right)$ and $\sigma \leftarrow \operatorname{Eval}\left(\mathrm{vk}, f, \sigma_{1}, \ldots, \sigma_{t}\right)$, then $|\sigma| \leq p(\lambda) \cdot \log t$.

Context Hiding. As an additional property, homomorphic signatures can be required to satisfy a privacy property called context-hiding [31]. Very intuitively, a homomorphic signature is context hiding if signatures on functions' outputs do not reveal information about the originally signed inputs. Here we recall the definition of context-hiding as given in [46, full version]. The notion in [46] generalizes the one proposed by Gorbunov, Vaikuntanathan and Wichs [77] to the setting of labeled programs. We defer the interested reader to the discussion in [46] for details on the differences and its relation to other prior context-hiding definitions.

Definition 2 (Context-Hiding [46]). An homomorphic signature scheme for labeled programs supports context-hiding if there exist additional PPT procedures $\tilde{\sigma} \leftarrow \operatorname{Hide}(\mathrm{vk}, m, \sigma)$ and $\operatorname{HVerify}(\mathrm{vk}, \mathcal{P}, \Delta, m, \sigma)$ such that:

- Correctness: For any (vk, sk $\leftarrow \operatorname{KeyGen}\left(1^{\lambda}, \mathcal{L}\right)$ and any tuple $(\mathcal{P}, \Delta, m, \sigma)$ such that $\operatorname{Ver}(\mathrm{vk}, \mathcal{P}, \Delta, m, \sigma)=1$, we have that if $\tilde{\sigma} \leftarrow \operatorname{Hide}(\mathrm{vk}, m, \sigma)$ then $\operatorname{HVerify}(\mathrm{vk}, \mathcal{P}, \Delta, m, \tilde{\sigma})=1$.

- Unforgeability: The signature scheme is secure when we replace the original verification algoritm Ver with HVerify in the security game.

- Context-Hiding Security: There is a simulator Sim such that, for any fixed (worst-case) choice of $(\mathrm{sk}, \mathrm{vk}) \in \operatorname{KeyGen}\left(1^{\lambda}, \mathcal{L}\right)$, any labeled program $\mathcal{P}=$ $\left(f, \tau_{1}, \ldots, \tau_{\ell}\right)$, data-set $\Delta$ and messages $m_{1}, \ldots, m_{\ell}$, there exists a function $\epsilon(\lambda)$ such that for any distinguisher $\mathcal{D}$ it holds

$$
\mid \operatorname{Pr}[\mathcal{D}(I, \operatorname{Hide}(\mathbf{v k}, m, \sigma))=1]-\operatorname{Pr}[\mathcal{D}(I, \operatorname{Sim}(\mathrm{sk}, \mathcal{P}, \Delta, m)=1] \mid=\epsilon(\lambda)
$$

where $I=\left(\mathrm{sk}, \mathrm{vk}, \mathcal{P}, \Delta,\left\{m_{i}, \sigma_{i}=\operatorname{Sign}\left(\mathrm{sk}, \Delta, \tau_{i}, m_{i}\right)\right\}_{i=1}^{\ell}\right), m=f\left(m_{1}, \ldots, m_{\ell}\right)$, $\sigma \leftarrow \operatorname{Eval}\left(\mathrm{vk}, \sigma_{1}, \ldots, \sigma_{\ell}\right)$, and the probabilities are taken over the randomness of Sign, Hide and Sim. If $\epsilon(\lambda)$ is negligible then the scheme is said to have 
statistical context-hiding, otherwise, if $\epsilon(\lambda)=0$, the scheme has perfect context-hiding.

Efficient Verification. We recall the notion of homomorphic signatures with efficient verification introduced in [49]. This property is interesting as it enables the use of homomorphic signatures for verifiable delegation of computation in the amortized model of [67]. The property states that the verification algorithm can be split in two phases: an offline phase where, given the verification key vk and a labeled program $\mathcal{P}$, one precomputes a concise key $\mathrm{vk}_{\mathcal{P}}$; an online phase in which $\mathbf{v k}_{\mathcal{P}}$ can be used to verify signatures w.r.t. $\mathcal{P}$ and any dataset $\Delta$. To achieve (amortized) efficiency, the idea is that $\mathbf{v k}_{\mathcal{P}}$ can be reused an unbounded number of times, and the online verification is cheaper than running $\mathcal{P}$. More formally:

Definition 3 (Efficient Verification). Let HSig = (KeyGen, Sign, Ver, Eval) be a homomorphic signature scheme for labeled programs. HSig satisfies efficient verification if there exist two additional algorithms (VerPrep, EffVer) such that:

$\operatorname{VerPrep}(\mathrm{vk}, \mathcal{P})$ : given in input the verification key $\mathrm{vk}$ and a labeled program $\mathcal{P}=$ $\left(f, \tau_{1}, \ldots, \tau_{n}\right)$, the verification preparation algorithm generates a concise verification key $\mathrm{vk}_{\mathcal{P}}$.

$\operatorname{EffVer}\left(\mathrm{vk}_{\mathcal{P}}, \Delta, m, \sigma\right):$ on input a verification key $\mathrm{vk}_{\mathcal{P}}$, a data set identifier $\Delta$, a message $m \in \mathcal{M}$ and a signature $\sigma$, the efficient verification algorithm outputs 0 (reject) or 1 (accept).

The above algorithms are required to satisfy the following two properties:

Correctness. Let $(\mathrm{sk}, \mathrm{vk}) \stackrel{\$}{\leftarrow} \operatorname{KeyGen}\left(1^{\lambda}, \mathcal{L}\right)$ be honestly generated keys, and $(\mathcal{P}, \Delta, m, \sigma)$ be any program/dataset/message/signature tuple with $\mathcal{P}$ such that $\operatorname{Ver}(\mathrm{vk}, \mathcal{P}, \Delta, m, \sigma)=1$. Then, for every $\mathrm{vk}_{\mathcal{P}} \stackrel{\$}{\leftarrow} \operatorname{VerPrep}(\mathrm{vk}, \mathcal{P}), \operatorname{Eff} \operatorname{Ver}\left(\mathrm{vk}_{\mathcal{P}}\right.$, $\Delta, m, \sigma)=1$ holds with all but negligible probability.

Amortized Efficiency. Let $\mathcal{P}=\left(f, \tau_{1}, \ldots, \tau_{n}\right)$ be a labeled program and $\Delta$ be a dataset identifier, let $\left(m_{1}, \ldots, m_{n}\right) \in \mathcal{M}^{n}$ be any vector of inputs, and let $t(n)$ be the time required to compute $f\left(m_{1}, \ldots, m_{n}\right)$. If $\mathrm{vk}_{\mathcal{P}} \leftarrow \operatorname{VerPrep}(\mathrm{vk}, \mathcal{P})$, then the time required for $\operatorname{EfVer}\left(\mathrm{vk}_{\mathcal{P}}, \Delta, m, \tau\right)$ is $t^{\prime}=o(t(n))$.

\subsubsection{Security Definitions}

At an intuitive level, a homomorphic signature is secure if an adversary, without knowledge of the secret key, can only come up with signatures that it obtained from the signer, or signatures that are obtained by running the Eval algorithm on signatures obtained from the legitimate signer. Formalizing this intuition turns out to be tricky and leaves space to different possibilities.

In what follows we present three different security notions for homomorphic signatures that we call semi-adaptive, adaptive, and strong adaptive, respectively. 
These notions share the same security experiment between an adversary $\mathcal{A}$ and a challenger, and the only difference lies in what is considered a forgery. The security experiment, denoted $\operatorname{Exp}_{\mathcal{A}, \text { HomSign }}^{\operatorname{HomUF}-C M A}(\lambda)$, proceeds as described below:

Key Generation The challenger runs $(v k, s k) \stackrel{\$}{\leftarrow} \operatorname{KeyGen}\left(1^{\lambda}, \mathcal{L}\right)$ and gives vk to $\mathcal{A}$.

Signing Queries $\mathcal{A}$ can adaptively submit queries of the form $(\Delta, \tau, m)$, where $\Delta$ is a data set identifier, $\tau \in \mathcal{L}$, and $m \in \mathcal{M}$. The challenger proceeds as follows:

- if $(\Delta, \tau, m)$ is the first query with the data set identifier $\Delta$, the challenger initializes an empty list $T_{\Delta}=\emptyset$ for $\Delta$.

- If $T_{\Delta}$ does not already contain a tuple $(\tau, \cdot)$ (i.e., $\mathcal{A}$ never asked for a query $(\Delta, \tau, \cdot))$, the challenger computes $\sigma \stackrel{\$}{\leftarrow} \operatorname{Sign}(\mathrm{sk}, \Delta, \tau, m)$, returns $\sigma$ to $\mathcal{A}$ and updates the list $T_{\Delta} \leftarrow T_{\Delta} \cup(\tau, m)$.

- If $(\tau, m) \in T_{\Delta}$ (i.e., the adversary had already queried the tuple $(\Delta, \tau, m))$, the challenger replies with the same signature generated before.

- If $T_{\Delta}$ contains a tuple $\left(\tau, m^{\prime}\right)$ for some message $m^{\prime} \neq m$, then the challenger ignores the query. Note that this means that a tuple $(\Delta, \tau, \cdot)$ can be queried only once.

Forgery The previous stage is executed until the adversary $\mathcal{A}$ outputs a tuple $\left(\mathcal{P}^{*}, \Delta^{*}, m^{*}, \sigma^{*}\right)$. The experiments outputs 1 if the tuple returned by $\mathcal{A}$ is a forgery, and 0 otherwise.

To complete the description of the experiment, it remains to define when a tuple $\left(\mathcal{P}^{*}, \Delta^{*}, m^{*}, \sigma^{*}\right)$ is considered a forgery. We give below three different forgery definitions; each of them yields a corresponding security notion for the homomorphic signature scheme.

Semi-Adaptive Secure Homomorphic Signatures. Informally speaking, in the semi-adaptive security game a forgery is one where either (1) the dataset $\Delta^{*}$ is "new" (i.e., no signing query $\left(\Delta^{*}, \cdot, \cdot\right)$ was ever made during the game), or (2) the claimed output $m^{*}$ of $\mathcal{P}^{*}$ is not the correct one. The crucial aspect of this definition is that to identify what is a correct output, one assumes that the adversary has fully specified the inputs of $\mathcal{P}^{*}$, namely $\mathcal{A}$ has asked for signatures on $\left(\Delta^{*}, \tau_{i}^{*}, m_{i}\right)$, for all $i=1$ to $n$. More formally,

Definition 4 (Semi-Adaptive Security). We define $\operatorname{Exp}_{\mathcal{A}, \text { HomSign }}^{\text {semi-Ad-UF }}(\lambda)$ as the security experiment which proceeds as $\operatorname{Exp}_{\mathcal{A}, \operatorname{Hom} \operatorname{Hom} \text { Sign }}^{\operatorname{Hom}}(\lambda)$ with the addition that the tuple $\left(\mathcal{P}^{*}:=\left(f^{*}, \tau_{1}^{*}, \ldots, \tau_{n}^{*}\right), \Delta^{*}, m^{*}, \sigma^{*}\right)$ returned by the adversary $\mathcal{A}$ is considered a forgery if $\operatorname{Ver}\left(\mathrm{vk}, \mathcal{P}^{*}, \Delta^{*}, m^{*}, \sigma^{*}\right)=1$ and either one of the following conditions hold: 
Type 1: The list $T_{\Delta^{*}}$ has not been initialised during the game.

Type 2: For all $i \in[n], \exists\left(\tau_{i}, m_{i}\right) \in T_{\Delta^{*}}$ and $m^{*} \neq f^{*}\left(m_{1}, \ldots, m_{n}\right)$.

Let $\operatorname{Adv}_{\mathcal{A}, \text { HomSign }}^{\text {semi-Ad-UF }}(\lambda)=\operatorname{Pr}\left[\operatorname{Exp}_{\mathcal{A}, \text { HomSign }}^{\text {semi-Ad-UF }}(\lambda)=1\right]$ be the advantage of $\mathcal{A}$ against the semi-adaptive security of scheme HomSign. We say that a homomorphic signature scheme HomSign is semi-adaptive secure (or simply secure) if for every PPT adversary $\mathcal{A}$ there exists a negligible function $\epsilon(\lambda)$ such that $\mathbf{A d v}_{\mathcal{A}, \mathrm{HomSign}}^{\text {semi-Ad-UF }}(\lambda)$ $\leq \epsilon(\lambda)$.

We stress that in the above security experiment the adversary $\mathcal{A}$ is restricted to produce Type 2 forgeries where all the inputs of the labeled program have been queried during the experiment. This notion works well for applications where the dataset is signed in one shot (as in the earlier proposals of homomorphic signatures [31]), or where one computes on the signed data only after the whole dataset has been filled up. In contrast, in those applications where the dataset is signed incrementally and one performs computations in between (e.g., in streaming applications), semi-adaptive security falls short of providing good guarantees. The issue is that in such a dynamic setting the adversary may claim a forgery with a labeled program containing a label $\tau^{*}$ that was not queried during the game. In this case, the input of $\mathcal{P}^{*}$ is no longer specified and defining whether the adversary's output is a forgery is not captured by Definition 4 . From the literature, we note that the schemes in [30, 69, 31, 77] are proven under a weaker version of semi-adaptive security where the messages of every dataset have to be queried all at once.2

Adaptive Secure Homomorphic Signatures. The issue of adversaries who claim programs in which some of the inputs are missing in the forgery stage was recognized earlier on by Freeman [63. To deal with this issue, he proposed a notion of "well-defined programs" which characterizes when the output of a program can be defined in spite of missing inputs. The idea is rather simple and intuitively says that a program is well-defined if the missing inputs do not change its outcome.

Definition 5 (Well-Defined Labeled Program [63]). A labeled program $\mathcal{P}^{*}=$ $\left(f^{*}, \tau_{1}^{*}, \ldots, \tau_{n}^{*}\right)$ is well-defined with respect to a list $T=\left\{\left(\tau_{i}, m_{i}\right)\right\}_{i}$ if one of the two following cases holds:

- $\forall i=1, \ldots, n:\left(\tau_{i}^{*}, m_{i}\right) \in T$.

- $\exists j \in\{1, \cdots, n\}$ s.t. $\left(\tau_{j}, \cdot\right) \notin T$, and for all possible choices of $\tilde{m}_{j} \in \mathcal{M}$ such that $\left(\tau_{j}, \cdot\right) \notin T f^{*}\left(m_{1}^{\prime}, \ldots, m_{n}^{\prime}\right)$ is the same, where $m_{i}^{\prime}=m_{i}$ for all $i$ s.t. $\left(\tau_{i}, m_{i}\right) \in T$ and $m_{i}^{\prime}=\tilde{m}_{i}$ otherwise.

${ }^{2}$ Actually, the authors of [77 mention that the proof of their scheme can be modified to hold under a definition with adaptive queries to data items, corresponding to the semi-adaptive security presented in this work. 
With the notion of well-defined programs, adaptive security can be defined as follows.

Definition 6 (Adaptive Security [63]). We define $\operatorname{Exp}_{\mathcal{A} \text {,HomSign }}^{\mathrm{Ad} \text {-UF }}(\lambda)$ as the security experiment which proceeds as $\operatorname{Exp}_{\mathcal{A}, \mathrm{HomSign}}^{\mathrm{HomUF}-\mathrm{CMA}}(\lambda)$ with the addition that the tuple $\left(\mathcal{P}^{*}:=\left(f^{*}, \tau_{1}^{*}, \ldots, \tau_{n}^{*}\right), \Delta^{*}, m^{*}, \sigma^{*}\right)$ returned by the adversary $\mathcal{A}$ is considered a forgery if $\operatorname{Ver}\left(\mathrm{vk}, \mathcal{P}^{*}, \Delta^{*}, m^{*}, \sigma^{*}\right)=1$ and either one of the following conditions hold:

Type 1: The list $T_{\Delta^{*}}$ has not been initialized during the game.

Type 2: $\mathcal{P}^{*}$ is well-defined with respect to $T_{\Delta^{*}}$, and $m^{*} \neq f^{*}\left(m_{1}^{\prime}, \ldots, m_{n}^{\prime}\right)$ where $m_{i}^{\prime}=m_{i}$ for all $i$ s.t. $\left(\tau_{i}, m_{i}\right) \in T_{\Delta^{*}}$ and $m_{i}^{\prime}=\tilde{m}$ (for some arbitrary $\tilde{m} \in \mathcal{M})$, otherwise.

Type 3: $\mathcal{P}^{*}$ is not well-defined with respect to $T_{\Delta^{*}}$.

Let $\operatorname{Adv}_{\mathcal{A}, \mathrm{HomSign}}^{\mathrm{Ad}-\mathrm{UF}}(\lambda)=\operatorname{Pr}\left[\operatorname{Exp}_{\mathcal{A}, \text { HomSign }}^{\mathrm{Ad}-\mathrm{UF}}(\lambda)=1\right]$ be the advantage of $\mathcal{A}$ against the adaptive security of scheme HomSign. We say that a homomorphic signature scheme HomSign is adaptive secure if for every PPT adversary $\mathcal{A}$ there exists a negligible function $\epsilon(\lambda)$ such that $\mathbf{A d v}_{\mathcal{A} \text {, HomSign }}^{\mathrm{Ad}-\mathrm{UF}}(\lambda) \leq \epsilon(\lambda)$.

Comparing the above definition of adaptive security with the semi-adaptive definition presented earlier, we note the following: Type 1 forgeries are identical in both definitions. Type 2 forgeries are similar: intuitively, they both capture the case when the adversary cheats on the result of $\mathcal{P}^{*}$, except that Definition 6 addresses the case of missing inputs by defining what is, in this case, a correct output (using the notion of well-defined program). Finally, Type 3 forgeries are introduced in Definition 6 to address the remaining case in which $\mathcal{P}^{*}$ may have different outputs, yet the forgery verifies correctly.

From the literature, the schemes in [63, 12, 47, 48, 45, 49, 46] are proven under the adaptive security notion presented above.

Strongly Adaptive Secure Homomorphic Signatures. The good of the adaptive definition given above is that it addresses the issue of labeled programs with unspecified inputs by modeling when an adversary is cheating. The modeling of Definition 6 however comes at the price of a rather cumbersome security definition. Well-defined programs are certainly not the most intuitive notion to work with. In addition, besides simplicity, the main issue with the above notion is that deciding whether the tuple returned by the adversary is a forgery may not be doable in polynomial time. Indeed, making this test would require to execute $f^{*}$ on all possible values of the missing inputs (that may be exponentially many). In the case when admissible functions are low-degree arithmetic circuits over a large field, it has been shown that well-defined programs can be tested probabilistically, and that Type 3 forgeries can be reduced to Type 2 ones [44. However, for general circuits the inefficient test issue remains and can generate 
troubles when proving the security of homomorphic signature schemes as well as when using them in larger protocols (as simply testing whether an adversary returned a forgery - wins - may not be doable in polynomial time).

To address this issue, in what follows we consider a stronger and much simpler security definition. This notion is obtained by extending the notion of semiadaptive security (Definition 4) with a very simple notion of Type 3 forgeries. The latter are just forgeries where the labeled program contains a "new" label. The formal definition follows.

Definition 7 (Strong Adaptive Security). We define $\operatorname{Exp}_{\mathcal{A}, \text { HomSign }}^{\text {strong-AA-UF }}(\lambda)$ as the security experiment which proceeds as $\operatorname{Exp}_{\mathcal{A}, \mathrm{HomSign}}^{\operatorname{HomUF}-\mathrm{CMA}}(\lambda)$ except that the tuple $\left(\mathcal{P}^{*}:=\left(f^{*}, \tau_{1}^{*}, \ldots, \tau_{n}^{*}\right), \Delta^{*}, m^{*}, \sigma^{*}\right)$ returned by the adversary $\mathcal{A}$ is considered a forgery if $\operatorname{Ver}\left(\mathrm{vk}, \mathcal{P}_{\Delta^{*}}^{*}, m^{*}, \sigma^{*}\right)=1$ and either one of the following conditions hold:

Type 1: The list $T_{\Delta^{*}}$ has not been initialized during the game.

Type 2: For all $i \in[n], \exists\left(\tau_{i}, m_{i}\right) \in T_{\Delta^{*}}$ and $m^{*} \neq f^{*}\left(m_{1}, \ldots, m_{n}\right)$.

Type 3 Strong: there exists $j \in\{1, \ldots, n\}$ such that $\left(\tau_{j}^{*}, \cdot\right) \notin T_{\Delta^{*}}$.

Let $\mathbf{A d v}_{\mathcal{A}, \mathrm{HomSign}}^{\text {strong-Ad-UF }}(\lambda)=\operatorname{Pr}\left[\operatorname{Exp}_{\mathcal{A}, \text { HomSign }}^{\text {strong-Ad-UF }}(\lambda)=1\right]$ be the advantage of $\mathcal{A}$ against the strong adaptive security of scheme HomSign. We say that a homomorphic signature scheme HomSign is strongly adaptive secure if for every PPT adversary $\mathcal{A}$ there exists a negligible function $\epsilon(\lambda)$ such that $\mathbf{A d v}_{\mathcal{A}, \text { HomSign }}^{\text {strong-Ad-UF }}(\lambda) \leq \epsilon(\lambda)$.

The security notion of Definition 7 now allows to detect forgeries in polynomial time, and is without doubt much simpler than Definition 6. Basically, this notion is the public-key equivalent of the security notion proposed by Gennaro and Wichs [70] for fully-homomorphic MACs (with some cosmetic changes due to the handling of multiple datasets).

Relation between security notions. We note that the three security definitions presented in this section are increasingly strong. Definition 6 is strictly stronger than Definition 4 ; while all forgeries in $\operatorname{Exp}_{\mathcal{A} \text {,HomSign }}^{\text {semi-Ad-UF }}(\lambda)$ are also forgeries in $\operatorname{Exp}_{\mathcal{A}, \text { HomSign }}^{\text {Ad-UF }}(\lambda)$, the converse is not true as any forgery in $\operatorname{Exp}_{\mathcal{A}, \mathrm{HomSign}}^{\mathrm{Ad}-\mathrm{UF}}(\lambda)$ where the labeled program $\mathcal{P}^{*}$ contains an unqueried label is not considered a forgery in $\operatorname{Exp}_{\mathcal{A}, \text { HomSign }}^{\text {semi-AdUF }}(\lambda)$.

Definition 7 is strictly stronger than Definition 6. In one direction, any Type 1 and Type 3 forgery in $\operatorname{Exp}_{\mathcal{A} \text {,HomSign }}^{\text {Ad-UF }}(\lambda)$ yields, respectively, a Type 1 and a Type 3 Strong forgery in $\mathbf{E x p}_{\mathcal{A} \text {,HomSign }}^{\text {strong-Ad-UF }}(\lambda)$, and a Type 2 forgery in $\operatorname{Exp}_{\mathcal{A}, \text { HomSign }}^{\text {Ad-UF }}(\lambda)$ becomes either a Type 2 forgery or a Type 3 Strong forgery in $\operatorname{Exp}_{\mathcal{A}, \text { HomSign }}^{\text {strong-Ad-UF }}(\lambda)$. In the other direction, there exist forgeries in experiment $\operatorname{Exp}_{\mathcal{A}, \text { HomSign }}^{\text {strong-Ad-UF }}(\lambda)$ that are not considered so in $\operatorname{Exp}_{\mathcal{A} \text {,HomSign }}^{\text {Ad-UF }}(\lambda)$. We show this by considering the following adversary $\mathcal{A}$. $\mathcal{A}$ asks signing queries $\left(\Delta, \tau_{1}, m_{1}\right),\left(\Delta, \tau_{2}, m_{2}\right)$ and obtains signatures $\sigma_{1}, \sigma_{2}$; it computes $\sigma^{*} \leftarrow \operatorname{Eval}\left(\mathrm{vk}, \times, \sigma_{1}, \sigma_{2}\right)$, and outputs $\left(\mathcal{P}^{*}:=\right.$ 
$\left.\left(f, \tau_{1}, \tau_{2}, \tau_{3}\right), \Delta, m_{1} \cdot m_{2}\right)$, where $f$ is the function $f(x, y, z)=x(y+z)-x z$. $^{3}$ As one can see, the output of $\mathcal{A}$ is a Type 3 Strong forgery, since $\tau_{3}$ is a label which has never been queried, while it is not a forgery in $\operatorname{Exp}_{\mathcal{A} \text {,HomSign }}^{\text {Ad-UF }}(\lambda)$, since $\mathcal{P}^{*}:=\left(f, \tau_{1}, \tau_{2}, \tau_{3}\right)$ is well-defined with respect to the set of queries $T_{\Delta}=$ $\left\{\left(\tau_{1}, m_{1}\right),\left(\tau_{2}, m_{2}\right)\right\}$, and $m_{1} \cdot m_{2}$ is the correct output.

In addition to the fact that the security notions are strictly separated, we also note that by using a counterexample such as the one above it is possible to show that previously proposed homomorphic signatures (e.g., [31, 49, 77]) are not strong adaptive secure.

\subsection{Generic Transformations from Semi-Adaptive to Strong Adaptive Security}

In this section we show a technique that allows one to turn a semi-adaptive unforgeable homomorphic signature into one that satisfies strong adaptive security. Specifically, our main result is stated in the following theorem:

Theorem 1. If $\Sigma$ is a semi-adaptive unforgeable fully (resp. leveled) homomorphic signature scheme for boolean circuits, then there exists a strong adaptive unforgeable homomorphic signature scheme $\widehat{\Sigma}$ that supports the same class of functions. Furthermore, if $\Sigma$ satisfies context-hiding (resp. efficient verification, composability) so does $\widehat{\Sigma}$.

The core of our result is a general transformation which shows how to combine a semi-adaptive secure scheme $\Sigma$ together with a semi-adaptive secure scheme $\Sigma_{\mathrm{OR}}$ that supports only OR operations over $\mathbb{Z}_{2}$. This combination yields a homomorphic signature scheme that is strong adaptive secure and supports the same class of functions supported by $\Sigma$.

Clearly, if $\Sigma$ supports the evaluation of boolean circuits, then $\Sigma_{\mathrm{OR}}$ can be instantiated using $\Sigma$. In this case, our result provides a way to bootstrap the security of $\Sigma$ from semi-adaptive to strong adaptive. This yields our main result above.

In the case where $\Sigma_{\mathrm{OR}}$ cannot be instantiated using $\Sigma$ (e.g., $\Sigma$ is not expressive enough), our transformation still provides a recipe to obtain strong adaptive security using a separate OR-homomorphic scheme. However, motivated by the lack of many candidates of OR-homomorphic signature schemes (concretely, 77] is the only available one), we investigated how to obtain a similar transformation by using schemes that have been studied more widely. Our second result is a transformation which can combine a semi-adaptive secure scheme $\Sigma$ together with

\footnotetext{
${ }^{3}$ Any other function where the third input cancels out would work. Furthermore, although in the given example it is trivial to recognize that $\mathcal{P}$ is well-defined, this may not be the case for general functions.
} 
a semi-adaptive secure linearly-homomophic signature $\Sigma_{\mathrm{LH}}$ that works for scalar messagef $4^{4}$ over a large ring, say $\mathbb{Z}_{p}$. This combination yields a homomorphic signature scheme that is strong adaptive secure and supports the same class of functions supported by $\Sigma$. A limitation of this second transformation is that it applies only to schemes that are leveled homomorphic (i.e., for circuits of bounded depth) as it requires to set $p>2^{d}$ where $d=\operatorname{poly}(\lambda)$ is the bound on circuits depth. On the other hand, the advantage is that strong adaptive security can be obtained by using linearly-homomorphic schemes, a class of constructions that has received significant attention, of which we know many constructions from several assumptions [12, 47, 13, 48, 63, 14, 45, 46], most of which are way more efficient in practice than [77. As for the efficiency of the scheme resulting from our transformations, it basically depends on the efficiency of the scheme one starts from. In the worst case, however, the efficiency loss is comparable to executing the original algorithms twice.

\subsubsection{Strong Adaptive Security from OR-Homomorphic Signatures}

Here we present our first transformation. The tools we start from are a homomorphic signature scheme $\Sigma:=(\Sigma$.KeyGen, $\Sigma$.Sign, $\Sigma$.Ver, $\Sigma$.Eval $)$ for a class $\mathcal{C}$ of (boolean or arithmetic) circuits, and a homomorphic signature $\Sigma_{\mathrm{OR}}:=$ $\left(\Sigma_{\mathrm{OR}}\right.$. KeyGen, $\Sigma_{\mathrm{OR}}$.Sign, $\Sigma_{\mathrm{OR}}$. Ver, $\Sigma_{\mathrm{OR}}$. Eval $)$ that works over message space $\mathbb{Z}_{2}$ and supports homomorphic OR operations. More precisely, $\Sigma_{\mathrm{OR}}$ must support circuits that are composed only of OR gates and have the same depth as those in $\mathcal{C}$.

Using $\Sigma$ and $\Sigma_{\text {OR }}$ in a black box way, we build a scheme $\widehat{\Sigma}$ which supports evaluation of circuits in $\mathcal{C}$. Moreover, assuming only semi-adaptive security of both $\Sigma$ and $\Sigma_{\mathrm{OR}}$, we show that $\widehat{\Sigma}$ is strong adaptive secure.

$\widehat{\Sigma} . \operatorname{KeyGen}\left(1^{\lambda}, \mathcal{L}\right)$. Run the key generation algorithms $(\mathrm{vk}, \mathrm{sk}) \leftarrow \operatorname{KeyGen}\left(1^{\lambda}, \mathcal{L}\right)$ and $\left(\mathrm{vk}_{\mathrm{OR}}, \mathrm{sk}_{\mathrm{OR}}\right) \leftarrow \Sigma_{\mathrm{OR}} \cdot \operatorname{KeyGen}\left(1^{\lambda}, \mathcal{L}\right)$, and output $(\hat{v k}, \hat{\mathrm{sk}}):=\left(\left(\mathrm{vk}, \mathrm{vk}_{\mathrm{OR}}\right)\right.$, $\left.\left(s k, s k_{O R}\right)\right)$.

$\widehat{\Sigma} \cdot \operatorname{Sign}(\hat{\mathrm{sk}}, \Delta, \tau, m)$. The signing algorithm uses the secret key to compute $\sigma \leftarrow$ $\operatorname{Sign}(\mathrm{sk}, \Delta, \tau, m)$ and $\sigma_{\mathrm{OR}} \leftarrow \Sigma_{\mathrm{OR}} \cdot \operatorname{Sign}\left(\mathrm{sk}_{\mathrm{OR}}, \Delta, \tau, 0\right)$, and outputs $\hat{\sigma}:=(\sigma$, $\left.\sigma_{\mathrm{OR}}\right)$.

Note that the OR-homomorphic component $\sigma_{\mathrm{OR}}$ of the signature signs the bit 0 . Although the usefulness of this component will become more clear in the security proof, the intuition is that this component keeps track of those labels that are used throughout the computation.

$\widehat{\Sigma}$.Eval(vk, $\left.f, \hat{\sigma}_{1}, \ldots, \hat{\sigma}_{n}\right)$. We describe the homomorphic evaluation of $f$ in a gateby-gate fashion, distinguishing the cases of unary and binary gates. One

\footnotetext{
${ }^{4}$ Namely, we do not need to work with vectors as most linearly-homomorphic signatures do.
} 
can easily see that the construction generalizes to $n$-ary gates. Describing the evaluation gate-by-gate is also useful to clearly see that our transformation allows for arbitrary composition of signatures (i.e., running $\widehat{\Sigma}$.Eval on outputs of $\widehat{\Sigma}$.Eval). At every gate $g$, one proceeds as follows.

Unary Gates. Let $g$ be an unary gate and let $\hat{\sigma}_{1}:=\left(\sigma_{1}, \sigma_{\mathrm{OR}, 1}\right)$ be the input. We compute the output signature $\hat{\sigma}_{\text {out }}:=\left(\sigma_{\text {out }}, \sigma_{\mathrm{OR}, \text { out }}\right)$ by computing $\sigma_{\text {out }} \leftarrow \Sigma$.Eval $\left(\mathrm{vk}, g, \sigma_{1}\right)$ and $\sigma_{\mathrm{OR}, \text { out }} \leftarrow \sigma_{\mathrm{OR}, 1}$. Basically, we evaluate $g$ over the $\Sigma$ component, while for the OR-homomorphic component we simply evaluate an identity function.

Binary Gates. Let $g$ be a binary gate and let $\hat{\sigma}_{1}:=\left(\sigma_{1}, \sigma_{\mathrm{OR}, 1}\right)$ and $\hat{\sigma}_{2}:=$ $\left(\sigma_{2}, \sigma_{\mathrm{OR}, 2}\right)$ be its two inputs. We compute the output signature $\hat{\sigma}_{\text {out }}:=$ $\left(\sigma_{\text {out }}, \sigma_{\mathrm{OR}, \text { out }}\right)$ by first evaluating $\sigma_{\text {out }} \leftarrow \Sigma$.Eval $\left(\mathrm{vk}, g, \sigma_{1}, \sigma_{2}\right)$ and then evaluating $\sigma_{\mathrm{OR}, \text { out }} \leftarrow \Sigma_{\mathrm{OR}}$. Eval $\left(\mathrm{vk}_{\mathrm{OR}}, \mathrm{OR}, \sigma_{\mathrm{OR}, 1}, \sigma_{\mathrm{OR}, 2}\right)$. Basically, we evaluate the binary $g$ over the $\Sigma$ components, while for the OR-homomorphic components we perform their homomorphic OR.

By proceeding over $f$ in a gate-by-gate fashion, eventually we obtain a signature $\hat{\sigma}:=\left(\sigma, \sigma_{\mathrm{OR}}\right)$, and $\hat{\Sigma}$. Eval returns $\hat{\sigma}$.

At this point, it is worth mentioning that the evaluation algorithm of our transformation generates $\left(\sigma, \sigma_{\mathrm{OR}}\right)$ such that $\sigma=\Sigma$. Eval $\left(\mathrm{vk}, f, \sigma_{1}, \ldots, \sigma_{n}\right)$ and $\sigma_{\mathrm{OR}}=\Sigma_{\mathrm{OR}}$. Eval $\left(\mathrm{vk}_{\mathrm{OR}}, f_{\mathrm{OR}}, \sigma_{\mathrm{OR}, 1}, \ldots, \sigma_{\mathrm{OR}, n}\right)$, where $f_{\mathrm{OR}}$ is an "OR version" of the circuit $f$ obtained by changing any unary gate with an identity gate and any binary gate with an OR gate.

$\widehat{\Sigma} . \operatorname{Ver}(\mathrm{vk}, \mathcal{P}, \Delta, m, \hat{\sigma})$. Parse $\mathcal{P}=\left(f, \tau_{1}, \ldots, \tau_{n}\right)$ and $\hat{\sigma}:=\left(\sigma, \sigma_{\mathrm{OR}}\right)$. Next, define $\mathcal{P}_{\mathrm{OR}}:=\left(f_{\mathrm{OR}}, \tau_{1}, \ldots, \tau_{n}\right)$, where $f_{\mathrm{OR}}$ is the circuit composed only of OR (and identity) gates, obtained from $f$ as described above. Then check if $\Sigma . \operatorname{Ver}(\mathrm{vk}, \mathcal{P}, \Delta, m, \sigma)=1$ and $\Sigma_{\mathrm{OR}} \cdot \operatorname{Ver}\left(\mathrm{vk}_{\mathrm{OR}}, \mathcal{P}_{\mathrm{OR}}, \Delta, 0, \sigma_{\mathrm{OR}}\right)=1$. If both the verification runs output 1 , then output 1 , otherwise output 0 .

In the following theorem we show that our generic scheme $\widehat{\Sigma}$ satisfies strong adaptive security, as long as the schemes $\Sigma$ and $\Sigma_{\mathrm{OR}}$ are only semi-adaptive secure.

Theorem 2. Assume that $\Sigma$ is a semi-adaptive secure homomorphic signature scheme for a class of circuits $\mathcal{C}$, and that $\Sigma_{\mathrm{OR}}$ is a semi-adaptive secure homomorphic signature with message space $\mathbb{Z}_{2}$ and supporting OR circuits. Then the scheme $\widehat{\Sigma}$ described above is a strong-adaptive secure homomorphic signature for $\mathcal{C}$. Furthermore, if both $\Sigma$ and $\Sigma_{\mathrm{OR}}$ satisfy context-hiding (resp. efficient verification, composability), then so does $\widehat{\Sigma}$.

Proof. First of all, it is easy to see that the correctness and succinctness of $\widehat{\Sigma}$ are granted by the respective properties of $\Sigma$ and $\Sigma_{\mathrm{OR}}$. Composability of signatures is also immediate by construction (as we observed in the $\widehat{\Sigma}$.Eval algorithm). Second, 
the proofs of context hiding and efficient verification are given slightly later in Lemmas 3 and 4 respectively.

Now we focus on the main result, which is the strong adaptive security of $\widehat{\Sigma}$.

Let $\operatorname{Exp}_{\mathcal{A}, \widehat{\Sigma}}^{\text {strong-Ad-UF }}(\lambda)$ be the strong adaptive security experiment. In this experiment we let $\mathrm{T}_{3}$ be the event that the adversary $\mathcal{A}$ comes up with a Type 3 Strong forgery. Then we have

$$
\begin{aligned}
\operatorname{Adv}_{\mathcal{A}, \widehat{\Sigma}}^{\text {strong-Ad-UF }}(\lambda) \leq & \mid \operatorname{Pr}\left[\operatorname{Exp}_{\mathcal{A}, \widehat{\Sigma}}^{\text {strong-Ad-UF }}(\lambda)=1, \mathrm{~T}_{3}\right]+ \\
& +\operatorname{Pr}\left[\operatorname{Exp}_{\mathcal{A}, \widehat{\Sigma}}^{\text {strong-Ad-UF }}(\lambda)=1 \mid \neg \mathrm{T}_{3}\right] \mid .
\end{aligned}
$$

To show that the above advantage is negligible for any $\mathrm{PPT} \mathcal{A}$, in the following lemmas we provide negligible bounds for both the quantities on the right hand side.

Lemma 1. For any PPT $\mathcal{A}$ there is a PPT $\mathcal{B}$ such that $\operatorname{Pr}\left[\operatorname{Exp}_{\mathcal{A}, \widehat{\Sigma}}^{\text {strong-Ad-UF }}(\lambda)=\right.$ $\left.1 \mid \neg \mathrm{T}_{3}\right]=\operatorname{Adv}_{\mathcal{B}, \Sigma}^{\text {semi-Ad-UF }}(\lambda)$.

Proof. If $\mathrm{T}_{3}$ does not happen, in $\operatorname{Exp}_{\mathcal{A}, \widehat{\Sigma}}^{\text {strong-Ad-UF }}(\lambda)$ the adversary $\mathcal{A}$ can win by coming up with a forgery of either Type 1 or Type 2 . In this case, we can easily build a reduction $\mathcal{B}$ which uses a forgery from $\mathcal{A}$ in order to break the semi-adaptive security of $\Sigma$.

$\mathcal{B}$ receives a public key vk for $\Sigma$ from its challenger, generates on its own a key-pair $\left(v_{\mathrm{OR}}, \mathrm{sk}_{\mathrm{OR}}\right)$ for $\Sigma_{\mathrm{OR}}$, and finally sends $\left(\mathrm{vk}, \mathrm{vk}_{\mathrm{OR}}\right)$ to $\mathcal{A}$. Whenever $\mathcal{A}$ makes a signing query, $\mathcal{B}$ uses sk ${ }_{\mathrm{OR}}$ to compute the signature component $\sigma_{\mathrm{OR}}$, while it obtains $\sigma$ by forwarding the same query to its challenger. So, it sends to $\mathcal{A}$ the signature $\hat{\sigma}:=\left(\sigma, \sigma_{\mathrm{OR}}\right)$. When $\mathcal{A}$ outputs a Type 1 (resp. Type 2) forgery $\left(\mathcal{P}^{*}:=\left(f^{*}, \tau_{1}^{*}, \ldots, \tau_{n}^{*}\right), \Delta^{*}, m^{*}, \hat{\sigma}^{*}:=\left(\sigma^{*}, \sigma_{\mathrm{OR}}^{*}\right)\right), \mathcal{B}$ outputs $\left(\mathcal{P}^{*}:=\right.$ $\left.\left(f^{*}, \tau_{1}^{*}, \ldots, \tau_{n}^{*}\right), \Delta^{*}, m^{*}, \sigma^{*}\right)$ as a Type 1 (resp. Type 2$)$ forgery for the scheme $\Sigma$.

Lemma 2. For any PPT $\mathcal{A}$ there is a PPT $\mathcal{B}^{\prime}$ such that $\operatorname{Pr}\left[\operatorname{Exp}_{\mathcal{A}, \widehat{\Sigma}}^{\text {strong-Ad-UF }}(\lambda)=\right.$ $\left.1 \mid \mathrm{T}_{3}\right]=\operatorname{Adv}_{\mathcal{B}^{\prime}, \Sigma_{\mathrm{OR}}}^{\mathrm{semi}-\mathrm{UA}-\mathrm{UF}}(\lambda)$.

Proof. This is the case where we use OR-homomorphic signatures in order to handle Type 3 forgeries. The reduction $\mathcal{B}^{\prime}$ works as follows.

Key Generation $\mathcal{B}^{\prime}$ receives a public key vk of of $\Sigma_{\mathrm{OR}}$ from its challenger $\mathcal{C}_{\Sigma_{\mathrm{OR}}}$; then $\mathcal{B}$ runs $(\mathrm{vk}, \mathrm{sk}) \leftarrow \Sigma$.KeyGen $\left(1^{\lambda}, \mathcal{L}\right)$ and sends $\mathrm{vk}=(\mathrm{vk}, \mathrm{vk}$ oR $)$ to $\mathcal{A}$.

Signing Queries Whenever $\mathcal{A}$ asks for a signature on $(\Delta, \tau, m), \mathcal{B}^{\prime}$ computes on its own the signature $\sigma \leftarrow \Sigma$.Sign(sk, $\Delta, \tau, m)$ and makes a signing query $(\Delta, \tau, 0)$ to $\mathcal{C}_{\Sigma_{\text {OR }}}$ getting back a signature $\sigma_{\text {OR }}$. It then sends to $\mathcal{A}$ the signature $\hat{\sigma}:=\left(\sigma, \sigma_{\mathrm{OR}}\right)$. 
Forgery Assume that $\mathcal{A}$ outputs a Type 3 Strong forgery $\left(\mathcal{P}^{*}=\left(f^{*}, \tau_{1}^{*}, \ldots, \tau_{n}^{*}\right)\right.$, $\left.\Delta^{*}, m^{*}, \hat{\sigma}^{*}\right)$. By definition of Type 3 Strong forgery, there exists a nonempty subset $\mathcal{J} \subset[n]$ of indices such that, for all $j \in \mathcal{J}$, the label $\tau_{j}^{*}$ has not been queried by $\mathcal{A}$ during the experiment $\operatorname{Exp}_{\mathcal{A}, \hat{\Sigma}}^{\text {strong-Ad-UF }}(\lambda)$. So, $\mathcal{B}^{\prime}$ chooses any index $\hat{j} \in \mathcal{J}$ (note that here we let $\mathcal{B}^{\prime}$ to make additional signing queries) and then makes the following signing queries to its challenger: $\left\{\left(\Delta^{*}, \tau_{j}^{*}, 0\right)\right\}_{j \in \mathcal{J} \backslash \hat{j}},\left(\Delta^{*}, \tau_{\hat{j}}^{*}, 1\right)$.

Let $\hat{\sigma}^{*}:=\left(\sigma^{*}, \sigma_{\mathrm{OR}}^{*}\right)$. By definition of Type 3 Strong forgery we have that $\Sigma_{\mathrm{OR}} \cdot \operatorname{Ver}\left(\mathrm{vk}_{\mathrm{OR}}, \mathcal{P}_{\mathrm{OR}}^{*}, \Delta^{*}, 0, \sigma_{\mathrm{OR}}^{*}\right)=1$, where $\mathcal{P}_{\mathrm{OR}}^{*}:=\left(f_{\mathrm{OR}}^{*}, \tau_{1}^{*}, \ldots, \tau_{n}^{*}\right)$ and $f_{\mathrm{OR}}^{*}$ is the OR version of the circuit $f^{*}$, as computed in the verification algorithm $\widehat{\Sigma}$.Ver.

$\mathcal{B}^{\prime}$ returns $\left(\mathcal{P}_{\mathrm{OR}}^{*}, \Delta^{*}, 0, \sigma_{\mathrm{OR}}^{*}\right)$ as its forgery.

To conclude the proof we claim that the above tuple is a Type 2 forgery against the scheme $\Sigma_{\mathrm{OR}}$. To see this, first notice that $\mathcal{B}^{\prime}$ has made signing queries for all the labels $\tau_{1}^{*}, \ldots, \tau_{n}^{*}$. Second, we claim that $0 \neq y$ where $y$ is the correct output obtained by computing $f_{\mathrm{OR}}^{*}$ on the inputs queried by $\mathcal{B}^{\prime}$ to its challenger, i.e., obtained by feeding 0 in all input wires of $f_{\mathrm{OR}}^{*}$ labeled by $\tau_{i}^{*}$ for $i \neq \hat{j}$, and 1 in the input wire labeled with $\tau_{\hat{j}}^{*}$.

To argue that $y \neq 0$, note that $f_{\mathrm{OR}}\left(x_{1}, \ldots, x_{n}\right)$ can be written as $\bigvee_{j=1}^{n} x_{j}$ and thus $y=x_{\hat{j}}=1$. Therefore, we have shown that whenever $\mathcal{A}$ breaks the strong adaptive security of $\widehat{\Sigma}$ by producing a Type 3 Strong forgery, $\mathcal{B}^{\prime}$ can break the semi-adaptive security of $\Sigma_{\mathrm{OR}}$ by producing a Type 2 forgery. This concludes the proof.

Now, we move to showing that our transformation preserves context hiding. The idea is that, since every signature of $\widehat{\Sigma}$ consists of a signature of the scheme $\Sigma$ and a signature of the scheme $\Sigma_{\mathrm{OR}}$, context-hiding on the two components easily implies context-hiding of the resulting signatures of $\widehat{\Sigma} \cdot 5$

Lemma 3. Assume that $\Sigma:=$ ( $\Sigma$.KeyGen, $\Sigma$.Sign, $\Sigma$.Ver, $\Sigma$.Eval) and $\Sigma_{\mathrm{OR}}:=$ $\left(\Sigma_{\mathrm{OR}} \cdot\right.$ KeyGen, $\Sigma_{\mathrm{OR}}$.Sign, $\Sigma_{\mathrm{OR}} \cdot$ Ver, $\Sigma_{\mathrm{OR}}$. Eval) are an homomorphic and OR homomorphic signature scheme respectively, and let both $\Sigma$ and $\Sigma_{\mathrm{OR}}$ support context hiding in the sense of Definition 2. Then, the homomorphic signature scheme $\widehat{\Sigma}$, output of the black box transformation described in Section 2.5.1 supports context hiding as well.

Proof. Since $\Sigma$ and $\Sigma_{\mathrm{OR}}$ support context hiding, we know that by definition there exist additional PPT procedures $\Sigma$.Hide, $\Sigma$.HVerify, $\Sigma_{\mathrm{OR}}$.Hide, $\Sigma_{\mathrm{OR}}$.HVerify which satisfy correctness and unforgeability in the sense of Definition2. Moreover, there

\footnotetext{
${ }^{5}$ It is interesting to note that, even if signatures $\sigma_{\mathrm{OR}}$ only sign 0 (so, they do not contain information on the actual messages), context hiding on $\Sigma_{\mathrm{OR}}$ is still required for the proof to go through.
} 
exist two simulators $\Sigma$.Sim and $\Sigma_{\mathrm{OR}}$. Sim that satisfy the context hiding security property. What we do is thus to show how to build the corresponding procedures $\widehat{\Sigma}$. Hide, $\widehat{\Sigma}$.HVerify and the simulator $\widehat{\Sigma}$.Sim for the homomorphic signature scheme $\widehat{\Sigma}$. Since any signature in $\widehat{\Sigma}$ is of the form $\hat{\sigma}:=\left(\sigma, \sigma_{\mathrm{OR}}\right)$, we define $\widehat{\Sigma} \cdot \operatorname{Hide}(\hat{\mathrm{vk}}, m, \hat{\sigma}):=\left(\Sigma . \operatorname{Hide}(\mathrm{vk}, m, \sigma), \Sigma_{\mathrm{OR}} \cdot \operatorname{Hide}\left(\mathrm{vk}_{\mathrm{OR}}, 0, \sigma_{\mathrm{OR}}\right)\right)$ and $\widehat{\Sigma} \cdot \mathrm{HVerify}(\hat{\mathrm{vk}}$ $\mathcal{P}, \Delta, m, \hat{\sigma}):=\Sigma$.HVerify $(\mathrm{vk}, \mathcal{P}, \Delta, m, \sigma) \wedge \Sigma_{\mathrm{OR}} . \mathrm{HVerify}\left(\mathrm{vk}_{\mathrm{OR}}, \mathcal{P}_{\mathrm{OR}}, \Delta, 0, \sigma_{\mathrm{OR}}\right)$.

It is not hard to see that correctness and unforgeability with respect to $\widehat{\Sigma}$. Hide and $\widehat{\Sigma}$.HVerify follows from the same properties of the corresponding procedures of $\Sigma$ and $\Sigma_{\text {OR }}$. Similarly, we define the simulator $\widehat{\Sigma}$.Sim as $\widehat{\Sigma} \cdot \operatorname{Sim}(\hat{s k}, \mathcal{P}, \Delta, m):=$ $\left(\Sigma \cdot \operatorname{Sim}(\mathrm{sk}, \mathcal{P}, \Delta, m), \Sigma_{\mathrm{OR}} \cdot \operatorname{Sim}\left(\mathrm{sk}_{\mathrm{OR}}, \mathcal{P}_{\mathrm{OR}}, \Delta, 0\right)\right)$. The context hiding of this simulator follows immediately from that of the two simulators that are used to define it.

Remark 1. It is interesting to mention that, although in the construction the signature component $\sigma_{\mathrm{OR}}$ signs 0 (and is thus independent of the actual message), a context hiding property for $\Sigma_{\mathrm{OR}}$ is still needed for the proof to go through. One reason for this is that in a homomorphic signature scheme signatures may change form (and distribution) after applying the evaluation algorithm, and context hiding actually requires that such output distribution is simulatable. That is why we need context hiding for $\Sigma_{\mathrm{OR}}$. Nevertheless, we note that in our specific case where the input messages are fixed, much simpler context hiding mechanisms could be applied, such as requiring signatures to be deterministically generated (e.g., using a PRF) and letting the simulator simply reconstruct such signatures and apply evaluation.

Finally, we show that efficient verification is also preserved. For this we observe that the verification algorithm of $\widehat{\Sigma}$ simply consists of running verification of both $\Sigma$ and $\Sigma_{\mathrm{OR}}$. Hence, if the latter two algorithms admit an efficient verification mechanism, this mechanism can also be used to obtain efficient verification in $\widehat{\Sigma}$.

Lemma 4. Assume that $\Sigma:=\left(\Sigma\right.$. KeyGen, $\Sigma$.Sign, $\Sigma$.Ver, $\Sigma$.Eval) and $\Sigma_{\mathrm{OR}}:=$ $\left(\Sigma_{\mathrm{OR}} \cdot\right.$ KeyGen $\Sigma_{\mathrm{OR}}$.Sign, $\Sigma_{\mathrm{OR}}$.Ver, $\Sigma_{\mathrm{OR}}$. Eval $)$ are an homomorphic and OR homomorphic signature scheme respectively, and let both $\Sigma$ and $\Sigma_{\mathrm{OR}}$ satisfy efficient verification in the sense of Definition 3 . Then, the homomorphic signature scheme $\widehat{\Sigma}$, output of the black box transformation described in Section 2.5.1 satisfies efficient verification as well.

Proof. Since $\Sigma$ and $\Sigma_{\mathrm{OR}}$ satisfy efficient verification, there exist additional algorithms ( $\Sigma$.VerPrep, $\Sigma$.EffVer) and $\left(\Sigma_{\mathrm{OR}}\right.$.VerPrep, $\Sigma_{\mathrm{OR}}$. EffVer $)$ which satisfy correctness and amortized efficiency in the sense of Definition 3. What we do is thus to show how to build the corresponding algorithms ( $\widehat{\Sigma}$.VerPrep, $\widehat{\Sigma}$. EffVer) for $\widehat{\Sigma}$. Since any signature in $\widehat{\Sigma}$ is of the form $\hat{\sigma}:=\left(\sigma, \sigma_{\text {OR }}\right)$, we define $\widehat{\Sigma}$.VerPrep $(\hat{v k}, \mathcal{P})$ as the algorithm that runs $\Sigma . \operatorname{VerPrep}(v k, \mathcal{P})$ and $\Sigma_{\mathrm{OR}} . \operatorname{VerPrep}\left(\mathrm{vk}_{\mathrm{OR}}, \mathcal{P}_{\mathrm{OR}}\right)$, and 
outputs $\hat{v k}_{\mathcal{P}}:=\left(\mathrm{vk}_{\mathcal{P}}, \mathrm{vk}_{\mathrm{OR}, \mathcal{P}_{\mathrm{OR}}}\right)$. Then we define $\widehat{\Sigma} . \operatorname{EfP} \operatorname{Ver}\left(\hat{v k}_{\mathcal{P}}, \Delta, m, \hat{\sigma}\right)$ as the algorithm that outputs 1 if and only if both $\Sigma$.EffVer $\left(\operatorname{vk}_{\mathcal{P}}, \Delta, m, \sigma\right)=1$ and $\Sigma_{\mathrm{OR}}$. EffVer $\left(\mathrm{vk}_{\mathrm{OR}, \mathcal{P}_{\mathrm{OR}}}, \Delta, 0, \sigma_{\mathrm{OR}}\right)=1$. It is trivial to see that the new algorithms are correct if so are the efficient verification algorithms of $\Sigma$ and $\Sigma_{\mathrm{OR}}$. The efficient verification property instead follows from the fact that $\widehat{\Sigma}$. EffVer simply runs two algorithms that are guaranteed to have each running time at most $o(t(n))$.

\subsubsection{Strong Adaptive Security from Linearly-Homomorphic Signatures}

Here we present our second transformation. This transformation is similar to the one of Section 2.5.1 it incorporates signatures from a second homomorphic signature scheme in order to handle Type 3 forgeries. However, instead of a ORhomomorphic scheme, here we use a linearly-homomorphic one. More in detail, our constructions takes in a homomorphic signature scheme $\Sigma:=$ ( $\Sigma$.KeyGen, $\Sigma$.Sign, $\Sigma$.Ver, $\Sigma$.Eval) that supports circuits of polynomial depth at most $d$ and fan-in $2:^{6}$ and an additive-homomorphic signature $\Sigma_{\mathrm{LH}}:=\left(\Sigma_{\mathrm{LH}} \cdot\right.$ KeyGen, $\Sigma_{\mathrm{LH}} \cdot \operatorname{Sign}$, $\Sigma_{\mathrm{LH}} \cdot$ Ver, $\Sigma_{\mathrm{LH}}$. Eval) that works over message space $\mathbb{Z}_{p}$, where $p>2^{d}$. Using $\Sigma$ and $\Sigma_{\mathrm{LH}}$ in a black box way, we build a scheme $\Sigma^{\prime}$ which supports the same circuits as $\Sigma$, and assuming only semi-adaptive security of $\Sigma$ and $\Sigma_{\mathrm{LH}}$, we show that $\Sigma^{\prime}$ is strong adaptive secure. The scheme $\Sigma^{\prime}$ is defined as follows:

$\Sigma^{\prime} \cdot \operatorname{KeyGen}\left(1^{\lambda}, \mathcal{L}\right)$. Run $(\mathrm{vk}, \mathrm{sk}) \leftarrow \Sigma$.KeyGen $\left(1^{\lambda}, \mathcal{L}\right)$ and $\left(\mathrm{vk}_{\mathrm{LH}}, \mathrm{sk}_{\mathrm{LH}}\right) \leftarrow \Sigma_{\mathrm{LH}} \cdot \operatorname{KeyGen}$ $\left(1^{\lambda}, \mathcal{L}\right)$, and output $\left(\mathrm{vk}^{\prime}, \mathrm{sk}^{\prime}\right):=\left(\left(\mathrm{vk}, \mathrm{vk}_{\mathrm{LH}}\right),\left(\mathrm{sk}, \mathrm{sk}_{\mathrm{LH}}\right)\right)$.

$\Sigma^{\prime} . \operatorname{Sign}\left(\mathbf{s k}^{\prime}, \Delta, \tau, m\right)$. The signing algorithm uses sk' to compute $\sigma \leftarrow \operatorname{Sign}(\mathrm{sk}, \Delta$, $\tau, m)$ and $\sigma_{\mathrm{LH}} \leftarrow \Sigma_{\mathrm{LH}} \cdot \operatorname{Sign}\left(\mathbf{s k}_{\mathrm{LH}}, \Delta, \tau, 0\right)$, and outputs $\sigma^{\prime}:=\left(\sigma, \sigma_{\mathrm{LH}}\right)$.

$\Sigma^{\prime} . \operatorname{Eval}\left(\mathrm{vk}^{\prime}, f, \sigma_{1}^{\prime}, \ldots, \sigma_{n}^{\prime}\right)$. As in the previous section, we describe the homomorphic evaluation of $f$ in a gate-by-gate fashion, distinguishing the cases of unary and binary gates. At every gate $g$, one proceeds as follows.

Unary Gates. Let $g$ be an unary gate and let $\sigma_{1}^{\prime}:=\left(\sigma_{1}, \sigma_{\mathrm{LH}, 1}\right)$ be the input. We compute the output signature $\sigma_{\text {out }}^{\prime}:=\left(\sigma_{\text {out }}, \sigma_{\mathrm{LH}, \text { out }}\right)$ by computing $\sigma_{\text {out }} \leftarrow \Sigma$.Eval $\left(\mathrm{vk}, g, \sigma_{1}\right)$ and $\sigma_{\mathrm{LH}, \text { out }} \leftarrow \sigma_{\mathrm{LH}, 1}$.

Binary Gates. Let $g$ be a binary gate and let $\sigma_{1}^{\prime}:=\left(\sigma_{1}, \sigma_{\mathrm{LH}, 1}\right)$ and $\sigma_{2}^{\prime}:=$ $\left(\sigma_{2}, \sigma_{\mathrm{LH}, 2}\right)$ be its two inputs. We compute the output signature $\sigma_{\text {out }}^{\prime}:=$ $\left(\sigma_{\text {out }}, \sigma_{\mathrm{LH}, \text { out }}\right)$ by first evaluating $\sigma_{\text {out }} \leftarrow \Sigma$.Eval $\left(\mathrm{vk}, g, \sigma_{1}, \sigma_{2}\right)$ and then evaluating $\sigma_{\mathrm{LH}, \text { out }} \leftarrow \Sigma_{\mathrm{LH}}$. Eval $\left(\mathrm{vk}_{\mathrm{LH}},+, \sigma_{\mathrm{LH}, 1}, \sigma_{\mathrm{LH}, 2}\right)$.

\footnotetext{
${ }^{6}$ We describe the transformation for fan-in 2 only for ease of exposition. It is easy to see that the same technique would work for constant fan-in $c$ setting up $p>c^{d}$.
} 
By proceeding over $f$ in a gate-by-gate fashion, eventually we obtain a signature $\sigma^{\prime}:=\left(\sigma, \sigma_{\mathrm{LH}}\right)$, and $\Sigma^{\prime}$.Eval returns $\sigma^{\prime}$. We note that the evaluation algorithm of our transformation generates $\left(\sigma, \sigma_{\mathrm{LH}}\right)$ such that $\sigma=$ $\Sigma$.Eval $\left(\mathrm{vk}, f, \sigma_{1}, \ldots, \sigma_{n}\right)$ and $\sigma_{\mathrm{LH}}=\Sigma_{\mathrm{LH}}$.Eval $\left(\mathrm{vk}_{\mathrm{LH}}, f_{+}, \sigma_{\mathrm{LH}, 1}, \ldots, \sigma_{\mathrm{LH}, n}\right)$, where $f_{+}$is an "additive version" of the circuit $f$ obtained by changing any unary gate with an identity gate and any binary gate with an additive gate.

$\Sigma^{\prime} . \operatorname{Ver}\left(\mathrm{vk}, \mathcal{P}, \Delta, m, \sigma^{\prime}\right)$. Parse $\mathcal{P}=\left(f, \tau_{1}, \ldots, \tau_{n}\right)$ and $\sigma^{\prime}:=\left(\sigma, \sigma_{\mathrm{LH}}\right)$. Next, define $\mathcal{P}_{+}:=\left(f_{+}, \tau_{1}, \ldots, \tau_{n}\right)$, where $f_{+}$is the additive circuit obtained from $f$ as described above. Then check if $\Sigma . \operatorname{Ver}(\mathrm{vk}, \mathcal{P}, \Delta, m, \sigma)=1$ and $\Sigma_{\mathrm{LH}} \cdot \operatorname{Ver}\left(\mathrm{vk}_{\mathrm{LH}}\right.$, $\left.\mathcal{P}_{+}, \Delta, 0, \sigma_{\mathrm{LH}}\right)=1$. If both the verification runs output 1 , then output 1 , otherwise output 0 .

In the following theorem we show that our generic scheme $\Sigma^{\prime}$ satisfies strong adaptive security, as long as the schemes $\Sigma$ and $\Sigma_{\mathrm{LH}}$ are only semi-adaptive secure

Theorem 3. Assume that $\Sigma$ is a semi-adaptive secure homomorphic signature scheme for circuits of polynomial depth at least $d$ and fan-in 2, and that $\Sigma_{\mathrm{LH}}$ is a semi-adaptive secure linearly-homomorphic signature scheme whose message space is $\mathbb{Z}_{p}$, with $p>2^{d}$. Then the scheme $\Sigma^{\prime}$ described above is a strong-adaptive secure homomorphic signature. Furthermore, if both $\Sigma$ and $\Sigma_{\mathrm{LH}}$ satisfy contexthiding (resp. efficient verification), then so does $\Sigma^{\prime}$.

Proof. First of all, it is easy to see that the correctness and succinctness of $\Sigma^{\prime}$ are granted by the respective properties of $\Sigma$ and $\Sigma_{\mathrm{LH}}$. The inheritance of contexthiding and efficient verification follows in the same way as for the scheme $\widehat{\Sigma}$ of the previous section.

Below we focus on the main result, which is the strong adaptive security of $\Sigma^{\prime}$. The structure of the proof is the same as the proof of Theorem 2 , the main difference is the handling of Type 3 Strong forgeries that here is done by using the semi-adaptive security of $\Sigma_{\mathrm{LH}}$.

Let $\operatorname{Exp}_{\mathcal{A}, \Sigma^{\prime}}^{\text {strong-UF }}(\lambda)$ be the strong adaptive security experiment. In this experiment we let $\mathcal{A}$ be the event that the adversary $\mathcal{A}$ comes up with a Type 3 Strong forgery. Then we have

$$
\begin{aligned}
\operatorname{Adv}_{\mathcal{A}, \Sigma^{\prime}}^{\text {strong-Ad-UF }}(\lambda) \leq & \operatorname{Pr}\left[\operatorname{Exp}_{\mathcal{A}, \Sigma^{\prime}}^{\text {strong-Ad-UF }}(\lambda)=1 \mid \mathcal{A}\right]+ \\
& \operatorname{Pr}\left[\operatorname{Exp}_{\mathcal{A}, \Sigma^{\prime}}^{\text {strong-Ad-UF }}(\lambda)=1 \mid \neg \mathcal{A}\right] .
\end{aligned}
$$

To show that the above advantage is negligible for any $\mathrm{PPT} \mathcal{A}$, in the following lemmas we provide negligible bounds for both the quantities on the right hand side.

Lemma 5. For any PPT $\mathcal{A}$ there is a PPT $\mathcal{B}$ such that $\operatorname{Pr}\left[\operatorname{Exp}_{\mathcal{A}, \Sigma^{\prime}}^{\text {strong-Ad-UF }}(\lambda)=\right.$ $1 \mid \neg \mathcal{A}]=\operatorname{Adv}_{\mathcal{B}, \Sigma}^{\text {semi-Ad-UF }}(\lambda)$. 
Chapter 2. Improving Definitions: A New Framework for Homomorphic Signatures

Proof. The proof is essentially the same as that of Lemma 1 and thus is omitted.

Lemma 6. For any PPT $\mathcal{A}$ there is a PPT $\mathcal{B}^{\prime}$ such that $\operatorname{Pr}\left[\operatorname{Exp}_{\mathcal{A}, \Sigma^{\prime}}^{\text {strong-Ad-UF }}(\lambda)=\right.$ $1 \mid \mathcal{A}]=\operatorname{Adv}_{\mathcal{B}^{\prime}, \Sigma_{\text {LH }}}^{\text {semi-Ad-UF }}(\lambda)$.

Proof. The reduction $\mathcal{B}^{\prime}$ works as follows.

Key Generation $\mathcal{B}^{\prime}$ receives a public key $v k_{\mathrm{LH}}$ of $\Sigma_{\mathrm{LH}}$ from its challenger $\mathcal{C}_{\Sigma_{\mathrm{LH}}}$; then $\mathcal{B}$ runs $(\mathrm{vk}, \mathrm{sk}) \leftarrow \Sigma$.KeyGen $\left(1^{\lambda}, \mathcal{L}\right)$ and sends $\mathrm{vk}^{\prime}=(\mathrm{vk}, \mathrm{vk} \mathrm{LH})$ to $\mathcal{A}$.

Signing Queries Whenever $\mathcal{A}$ asks for a signature on $(\Delta, \tau, m), \mathcal{B}^{\prime}$ computes on its own the signature $\sigma \leftarrow \Sigma$.Sign(sk, $\Delta, \tau, m)$ and makes a signing query $(\Delta, \tau, 0)$ to $\mathcal{C}_{\Sigma_{\mathrm{LH}}}$ getting back a signature $\sigma_{\mathrm{LH}}$. It then sends to $\mathcal{A}$ the signature $\sigma^{\prime}:=\left(\sigma, \sigma_{\mathrm{LH}}\right)$.

Forgery Assume that $\mathcal{A}$ outputs a Type 3 Strong forgery $\left(\mathcal{P}^{*}=\left(f^{*}, \tau_{1}^{*}, \ldots, \tau_{n}^{*}\right)\right.$, $\left.\Delta^{*}, m^{*},\left(\sigma^{*}\right)^{\prime}\right)$. By definition of Type 3 Strong forgery, there exists a nonempty subset $\mathcal{J} \subset[n]$ of indices such that, for all $j \in \mathcal{J}$, the label $\tau_{j}^{*}$ has not been queried by $\mathcal{A}$ during the experiment $\operatorname{Exp}_{\mathcal{A}, \Sigma^{\prime}}^{\text {strong-Ad-UF }}(\lambda)$. So, $\mathcal{B}^{\prime}$ chooses any index $\hat{j} \in \mathcal{J}$ and then makes the following additional signing queries to its challenger: $\left\{\left(\Delta^{*}, \tau_{j}^{*}, 0\right)\right\}_{j \in \mathcal{J} \backslash \hat{j}},\left(\Delta^{*}, \tau_{\hat{j}}^{*}, 1\right)$.

Let $\left(\sigma^{*}\right)^{\prime}:=\left(\sigma^{*}, \sigma_{\mathrm{LH}}^{*}\right)$. By definition of Type 3 Strong forgery we have that $\Sigma^{\prime} \cdot \operatorname{Ver}\left(\mathrm{vk}_{\mathrm{LH}}, \mathcal{P}_{+}^{*}, \Delta^{*}, 0, \sigma_{\mathrm{LH}}^{*}\right)=1$, where $\mathcal{P}_{+}^{*}:=\left(f_{+}^{*}, \tau_{1}^{*}, \ldots, \tau_{n}^{*}\right)$ and $f_{+}^{*}$ is the additive version of the circuit $f^{*}$, as computed in the verification algorithm $\Sigma^{\prime}$. Ver.

Finally, $\mathcal{B}^{\prime}$ returns $\left(\mathcal{P}_{+}^{*}, \Delta^{*}, 0, \sigma_{\mathrm{LH}}^{*}\right)$ as its forgery.

To conclude the proof we claim that the above tuple is a Type 2 forgery against the scheme $\Sigma_{\mathrm{LH}}$. To see this, first notice that $\mathcal{B}^{\prime}$ has made signing queries for all the labels $\tau_{1}^{*}, \ldots, \tau_{n}^{*}$. Second, we claim that $0 \neq y$ where $y$ is the correct output obtained by computing $f_{+}^{*}$ on the inputs queried by $\mathcal{B}^{\prime}$ to its challenger, i.e., obtained by feeding 0 in all input wires of $f_{+}^{*}$ labeled by $\tau_{i}^{*}$ for $i \neq \hat{j}$, and 1 in the input wire labeled with $\tau_{\hat{j}}^{*}$.

To argue that $y \neq 0$, note that $f_{+}^{*}\left(x_{1}, \ldots, x_{n}\right)$ can be written as $\sum_{j=1}^{n} \gamma_{\tau_{i}^{*}} \cdot x_{i}$, and (by our choice of the $x_{i}$ 's) $y=\gamma_{\tau_{j}^{*}}$. Moreover, for every $i$ we have that $1 \leq \gamma_{\tau_{i}^{*}}<2^{d}$. The latter fact is granted by our assumption that the circuits of $\Sigma^{\prime}$ (and thus $f_{+}^{*}$ too) have fan-in 2 and depth at most $d$. Hence using our choice of $p>2^{d}$ we also have that $y=\gamma_{\tau_{\hat{j}}^{*}} \neq 0 \bmod p$.

Therefore, we have shown that whenever $\mathcal{A}$ breaks the strong adaptive security of $\Sigma^{\prime}$ by producing a Type 3 Strong forgery, $\mathcal{B}^{\prime}$ can break the semi-adaptive security of $\Sigma_{\mathrm{LH}}$ by producing a Type 2 forgery. This concludes the proof. 


\section{Improving Efficiency: Linearly Homomorphic Signatures with Sublinear Public Keys via APHFs}

In this Chapter we present our results in terms of efficiency of Homomorphic Signatures. After providing an intuitive description of Asymmetric Programmable Hash Functions (APHFs), the new cryptographic tool that we introduced which allows to mimic random oracle properties, we will present an overview of our advancements in terms of efficiency of (homomorphic) signatures. Then, we will present all the technical details regarding APHFs and our constructions of both linearly homomorphic signatures and digital signatures with sublinear public keys.

As we already mentioned in the previous chapters, the notion of homomorphic signatures was first introduced by Johnson et al. [85]. Since then several schemes have been proposed: the first schemes were homomorphic only for linear functions over vector spaces [30, 69, 12, 32, 47, 48, 63, 13, 45, 14, 92] and have nice applications to network coding and proofs of retrievability. More recent works proposed realizations that can support more expressive functionalities such as polynomials [31, 49] or general circuits of bounded polynomial depth [77, 38].

Despite the significant research work in the area, it is striking that all the existing homomorphic signature schemes that are proven secure in the standard model [12, 47, 48, 63, 13, 14, 92, 49, 77, 38] suffer from a public key that is at least linear in the size $N$ of the signed datasets. On one hand, the cost of storing such large public key can be, in principle, amortized since the key can be reused for multiple datasets. On the other hand, this limitation still represents a challenging open question from both a theoretical and a practical point of view. From a practical perspective, a linear public key might be simply unaffordable by a user Bob who has limited storage capacity. From a theoretical point of view, considered the state-of-the-art, it seems unclear whether achieving a standardmodel scheme with a key of length $o(N)$ is possible at all. Technically speaking, indeed, all these schemes in the standard model somehow rely on a public key as large as one dataset for simulation purposes. This essentially hints that any 
Chapter 3. Improving Efficiency: Linearly Homomorphic Signatures with Sublinear Public Keys via APHFs

solution for this problem would require a novel proof strategy.

Said in another way, with respect to the size of the public keys of homomorphic signatures schemes, there is an open question which represents an interesting challenge in the field:

Do homomorphic signatures with sublinear public key exist in the standard model?

\subsection{An Overview of Our Contribution}

We solve the above open problem by proposing the first standard-model homomorphic signature scheme that achieves a public key whose size is sub-linear in the maximal size $N$ of the supported datasets; moreover, our scheme is context-hiding secure. Slightly more in detail, we show how to use a new cryptographic tool that we introduced, called Asymmetric Programmable Hash Functions (APHFs), in a generic fashion to construct a linearly-homomorphic signature scheme based on bilinear maps that can sign datasets, each consisting of up to $N$ vectors of dimension $T$. The public key of our scheme mainly consists of the public hash keys of two APHFs. By instantiating these using (one of) our concrete realizations we obtain a linearly-homomorphic signature with a public key of length $O(\sqrt{N}+\sqrt{T})$. We stress that ours is also the first linearly-homomorphic scheme where the public key is sub-linear in the dimension $T$ of the signed vectors. Concretely, if one considers applications with datasets of 1 million of elements and a security parameter of 128 bits, previous solutions (e.g., [48, 13]) require a public key of at least $32 \mathrm{MB}$, whereas our solution simply works with a public key below $100 \mathrm{~KB}$.

\subsection{1 (Asymmetric) Programmable Hash Functions}

In this section we start discussing the notion of programmable hash functions, and then we present our extension, that is Asymmetric Programmable Hash Functions. Finally, we present their applications to regular and linearly-homomorphic signature schemes.

Programmable Hash Functions. Programmable Hash Functions (PHFs) were introduced by Hofheinz and Kiltz [83] as an information theoretic tool to "mimic" the behavior of a random oracle in finite groups. In a nutshell, a PHF H is an efficiently computable function that maps suitable inputs (e.g., binary strings) into a group $\mathbb{G}$, and can be generated in two different, indistinguishable, ways. In the standard modality, $\mathrm{H}$ hashes inputs $X$ into group elements $\mathrm{H}(X) \in \mathbb{G}$. When generated in trapdoor mode, a trapdoor allows one to express every output in terms of two (user-specified) elements $g, h \in \mathbb{G}$, i.e., one can compute two integers $a_{X}, b_{X}$ such that $\mathrm{H}(X)=g^{a_{X}} h^{b_{X}}$. Finally, $\mathrm{H}$ is programmable in the sense that 
it is possible to program the behavior of $\mathrm{H}$ so that its outputs contain (or not) $g$ with a certain probability. More precisely, $\mathrm{H}$ is said $(m, n)$-programmable if for all disjoint sets of inputs $\left\{X_{1}, \ldots, X_{m}\right\}$ and $\left\{Z_{1}, \ldots, Z_{n}\right\}$, the joint probability that $\forall i, a_{X_{i}}=0$ and $\forall j, a_{Z_{j}} \neq 0$ is significant (e.g., $1 /$ poly $(\lambda)$ ). Programmability turns out to be particularly useful in several security proofs. For instance, consider a security proof where a signature on $\mathrm{H}(X)$ can be simulated as long as $a_{X}=0$ (i.e., $g$ does not appear) while a forgery on $\mathrm{H}(Z)$ can be successfully used if $a_{Z} \neq 0$ (i.e., $g$ does appear). Then one could rely on an $(m, 1)$-programmability of $\mathbf{H}$ to "hope" that all the queried messages $X_{1}, \ldots, X_{m}$ are simulatable, i.e., $\forall i, a_{X_{i}}=0$, while the forgery message $Z$ is not, i.e., $a_{Z} \neq 0$. PHFs essentially provide a nice abstraction of the so-called partitioning technique used in many cryptographic proofs.

Asymmetric Programmable Hash Functions. We introduce the notion of asymmetric programmable hash functions (APHFs) which modifies the original notion of PHFs [83] in two main ways. First, an APHF $\mathrm{H}$ maps inputs into a bilinear group $\mathbb{G}$ and is only secretly computable. At the same time, an isomorphic copy of $\mathrm{H}$ can be publicly computed in the target group $\mathbb{G}_{T}$, i.e., anyone can compute $e(\mathrm{H}(X), g) 1_{1}^{1}$ Second, when generated in trapdoor mode, for two given group elements $g, h \in \mathbb{G}$ such that $h=g^{z}$, the trapdoor allows one to write every $\mathrm{H}(X)$ as $g^{c_{X}(z)}$ for a degree- $d$ polynomial $c_{X}(z)$.

We define two main programmability properties of APHFs. The first one is an adaptation of the original programmability notion, and it says that $\mathrm{H}$ is $(m, n, d)$ programmable if it is $(m, n)$-programmable as before except that, instead of looking at the probability that $a_{X}=0$, one now looks at whether $c_{X, 0}=0$, where $c_{X, 0}$ is the coefficient of the degree- 0 term of the polynomial $c_{X}(\cdot)$ obtained using the trapdoor 2 The second programmability property is new and is called programmable pseudorandomness. Roughly speaking, programmable pseudorandomness says that one can program $\mathrm{H}$ so that the values $g^{c_{X, 0}}$ look random to any polynomially-bounded adversary who observes the public hash key and the outputs of $\mathrm{H}$ on a set of adaptively chosen inputs. This functionality turns out to be useful in security proofs where one needs to cancel some random values for simulation purposes (we explain this in slightly more detail later in the introduction). In other words, programmable pseudorandomness provides another random-oracle-like property for standard model hash functions, that is to "hide" a PRF inside the hash function. This is crucial in our security proofs, and we believe it can have further applications.

Applications. In principle, secretly computable PHFs seem less versatile than regular PHFs. In this work, however, we show that, for applications such as digital signatures, APHFs turn out to be more powerful than their publicly computable counterparts. Specifically, we show how to use APHFs to realize both regular and

\footnotetext{
${ }^{1}$ Because of such asymmetric behavior we call these functions "asymmetric".

${ }^{2}$ For $d=1$, this is basically the same form of programmability of 83 .
} 
linearly-homomorphic signatures secure in the standard model. Next, we show efficient realizations of APHFs that, when plugged in our generic constructions, yield new and existing schemes that improve the state-of-the-art in the following way. First, we obtain the first linearly homomorphic signature scheme, secure in the standard model, achieving a public key which is sub-linear in both the dataset size and the dimension of the signed vectors. Second, we obtain regular signature schemes, matching the efficiency of the ones in [116], thus providing the shortest signatures in the standard model with a public key shorter than in [82].

More in detail, we show

- linearly-homomorphic signatures: we obtain the first standard-model scheme in which the public key is sub-linear in both the dataset size and the dimension of the signed vectors.

- regular signatures: we obtain standard-model signatures that achieve the same efficiency as the scheme of Yamada et al. [116].

We have already discussed the first result on linearly-homomorphic signatures in the beginning of this chapter. In the following we elaborate more on the second application to regular digital signatures.

Short Signatures from Bilinear Maps in the Standard Model. Hofheinz and Kiltz [83] proposed efficient realizations of PHFs, and showed how to use them to obtain black-box proofs of several cryptographic primitives. Among these applications, they use PHFs to build generic, standard-model, signature schemes from the Strong RSA problem and the Strong q-Diffie Hellman problem. Somewhat interestingly, these schemes (in particular the ones over bilinear groups) can enjoy very short signatures. The remarkable contribution of the generic construction in [83] is that signatures can be made short by reducing the size $\rho$ of the randomness used (and included) in the signature so that $\rho$ can go beyond the birthday bound. Precisely, by using an $(m, 1)$-programmable hash function, $m$ can control the size of the randomness so that the larger is $m$, the smaller is the randomness. However, although this would call for $(m, 1)$-PHFs with a large $m$, the original work [83] described PHFs realizations that are only $(2,1)$-programmable $3^{3}$

Later, Hofheinz, Jager and Kiltz [82] showed constructions of $(m, 1)$-PHFs for any $m \geq 1$. By choosing a larger $m$, these new PHFs realizations yield the shortest known signatures in the standard model. On the negative side, however, this also induces much larger public keys. For instance, to obtain a signature of 302 bits from bilinear maps, they need a public key of more than $8 \mathrm{MB}$. The reason of such inefficiency is that their realizations of (deterministic) $(m, 1)$-PHFs have keys of length $O\left(m^{2} \ell\right)$, where $\ell$ is the bit size of the inputs. In a subsequent work, Yamada et al. [116] improved on this aspect by proposing a signature scheme with a public key of length $O(m \sqrt{\ell})$. Their solution followed a different approach: instead of relying on $(m, 1)$-PHFs they obtained the signature by applying the

\footnotetext{
${ }^{3} 83$ gives also a (1, poly)-programmable PHF which allows for different applications.
} 
Chapter 3. Improving Efficiency: Linearly Homomorphic Signatures with Sublinear Public Keys via APHFs

\begin{tabular}{|c|c|c|c|c|c|c|}
\hline \multirow[t]{2}{*}{ Signature Scheme } & & \multicolumn{2}{|c|}{ Sig. Size (bits) } & \multicolumn{3}{|c|}{ Public Key Size (KB) } \\
\hline & & $\lambda=80$ & $\lambda=128$ & & $\lambda=80$ & $\lambda=128$ \\
\hline 113 Waters $(\mathrm{CDH})$ & & 320 & 512 & $\left|\mathbb{G}_{1}\right|+(\ell+3)\left|\mathbb{G}_{2}\right|$ & 6.5 & 16.6 \\
\hline \begin{tabular}{l|l}
28 & Boneh-Boyen ( $q$-SDH
\end{tabular} & & 320 & 512 & $\begin{array}{ll} & 2\left|\mathbb{G}_{2}\right| \\
\end{array}$ & 0.08 & 0.13 \\
\hline 83] $\mathrm{Sig}_{\mathrm{q}-\mathrm{SDH}}\left[\mathrm{H}_{\mathrm{Wat}}\right]$ & $(m=2)$ & 230 & 350 & $(\ell+1)\left|\mathbb{G}_{1}\right|+\left|\mathbb{G}_{2}\right|$ & 3.3 & 8.3 \\
\hline 82$] \mathrm{Sig}_{\mathrm{q}-\mathrm{SDH}}\left[\mathrm{H}_{\mathrm{cfs}}\right]$ & $(m=8)$ & 200 & 302 & $\left(16 m^{2} \ell\right)\left|\mathbb{G}_{1}\right|+\left|\mathbb{G}_{2}\right|$ & 3276.8 & 8388.7 \\
\hline$\Sigma_{\mathrm{q}-\mathrm{SDH}}\left[\mathrm{H}_{\mathrm{acfs}}\right]$ & $(m=8)$ & 200 & 302 & $4 m\lceil\sqrt{\ell}\rceil\left(\left|\mathbb{G}_{1}\right|+\left|\mathbb{G}_{2}\right|\right)+\left|\mathbb{G}_{2}\right|$ & 25 & 49.2 \\
\hline $82 \mathrm{Sig}_{\mathrm{q}-\mathrm{DH}}\left[\mathrm{H}_{\mathrm{Wat}}, \mathrm{H}_{\mathrm{Wat}}\right]$ & $(m=2)$ & 230 & 350 & $\overline{(\ell+1)\left|\mathbb{G}_{1}\right|+(\rho+1)\left|\mathbb{G}_{2}\right|}$ & 4.9 & 11.2 \\
\hline $82 \mathrm{Sig}$-DH $\left[\mathrm{H}_{\mathrm{cfs}}, \mathrm{H}_{\mathrm{Wat}}\right]$ & $(m=8)$ & 200 & 302 & $\left(16 m^{2} \ell\right)\left|\mathbb{G}_{1}\right|+(\rho+1)\left|\mathbb{G}_{2}\right|$ & 3278.4 & 8391.6 \\
\hline [116] $\Sigma_{\mathrm{q}-\mathrm{DH}}\left[\mathrm{H}_{\mathrm{acfs}}, \mathrm{H}_{\mathrm{Wat}}\right]$ & $(m=8)$ & 200 & 302 & $4 m\lceil\sqrt{\ell}\rceil\left(\left|\mathbb{G}_{1}\right|+\left|\mathbb{G}_{2}\right|\right)+(\rho+1)\left|\mathbb{G}_{2}\right|$ & 26.6 & 52.2 \\
\hline
\end{tabular}

Table 3.1: Comparison between different standard-model signature schemes from bilinear maps. The shown values consider: (i) security at both $\lambda=80$ and $\lambda=128$ against adversaries seeing up to $q=2^{30}$ signatures; (ii) an implementation with TypeIII pairings where $\left|\mathbb{G}_{1}\right|=p=2 \lambda$ and $\left|\mathbb{G}_{2}\right|=2\left|\mathbb{G}_{1}\right|$; (iii) messages of $2 \lambda$ bits so as to provide collision-resistance for $\lambda$ bits of security; (iv) the size of the randomness $\rho=\log q+\left\lceil\frac{k}{m}\right\rceil$ according to the analysis in [83]. We considered an implementation of Waters' scheme which optimizes the signature size. Above Exp denotes the cost of an exponentiation in $\mathbb{G}_{1}$. The grey rows point out the results from this work.

Naor's transformation [29] to a new identity-based key encapsulation mechanism (IBKEM).

Our Results. Our results are mainly two. First, we revisit the generic signature constructions of [83, 82] in order to work with $(m, 1, d)$-APHFs. Our generic construction is very similar to that in [83, 82], and, as such, it inherits the same property: the larger is $m$, the shorter can be the randomness.

Second we show the construction of an APHF, $\mathrm{H}_{\text {acfs }}$, that is $(m, 1,2)$ - programmable and has a hash key consisting of $O(m \sqrt{\ell})$ group elements. By plugging $\mathrm{H}_{\text {acfs }}$ into our generic construction we immediately obtain standard-model signatures that achieve the same efficiency as the scheme of Yamada et al. [116]. Namely, they are the shortest standard model signature schemes with a public key of length $O(m \sqrt{\ell})$, that concretely allows for signatures of 302bits and a public key of $50 \mathrm{~KB}$. One of our two schemes recover the one in [116]. In this sense we provide a different conceptual approach to construct such signatures. While Yamada et al. obtained this result by going through an IBKEM, our solution revisits the original Hofheinz-Kiltz's idea of applying programmable functions.

We provide a detailed comparison of the schemes in Table 3.1 .

On the Power of Secretly-Computable PHFs. The main technical idea underlying this result is a new proof technique that builds on asymmetric hash functions with programmable pseudo-randomness. We illustrate the technique via a toy example inspired by our linearly-homomorphic signature scheme. The scheme works over asymmetric bilinear groups $\mathbb{G}_{1}, \mathbb{G}_{2}$, and with an APHF $\mathrm{H}$ : $[N] \rightarrow \mathbb{G}_{1}$ that has programmable pseudo-randomness w.r.t. $d=1$. To sign a 
Chapter 3. Improving Efficiency: Linearly Homomorphic Signatures with Sublinear Public Keys via APHFs

random message $M \in \mathbb{G}_{1}$ w.r.t. a label $\tau$, one creates the signature

$$
S=(\mathrm{H}(\tau) \cdot M)^{1 / z}
$$

where $z$ is the secret key. The signature is linearly-homomorphic $-S_{1} S_{2}=$ $\left(\mathrm{H}\left(\tau_{1}\right) \mathrm{H}\left(\tau_{2}\right) M\right)^{1 / z}$, for $M=M_{1} M_{2}$ - and it can be efficiently checked using a pairing $-e\left(S, g_{2}^{z}\right)=\prod_{i} e\left(\mathrm{H}\left(\tau_{i}\right), g_{2}\right) e\left(M, g_{2}\right)$ - and by relying on that $e\left(\mathrm{H}(\cdot), g_{2}\right)$ is publicly computable.

The first interesting thing to note is that having $\mathrm{H}$ secretly computable is necessary: if $\mathrm{H}$ is public the scheme could be easily broken, e.g., choose $M^{*}=$ $\mathrm{H}(\tau)^{-1}$. Let us now show how to prove its security assuming that we want to do a reduction to the following assumption: given $g_{1}, g_{2}, g_{2}^{z}$, the challenge is to compute $W^{1 / z} \in \mathbb{G}_{1}$ for $W \neq 1$ of adversarial choice. Missing $g_{1}^{z}$ seems to make hard the simulation of signatures since $M, S \in \mathbb{G}_{1}$. However, we can use the trapdoor generation of $\mathrm{H}$ for $d=1$ (that for asymmetric pairings takes $g_{1}, h_{1}=$ $g_{1}^{y_{1}}, g_{2}, h_{2}=g_{2}^{y_{2}}$ and allows to express $\left.\mathrm{H}(X)=g_{1}^{c_{X}\left(y_{1}, y_{2}\right)}\right)$, by plugging $h_{1}=1, h_{2}=$ $g_{2}^{z}$. This allows to write every output as $\mathrm{H}(\tau)=g_{1}^{c_{\tau}(z)}=g_{1}^{c_{\tau, 0}+c_{\tau, 1} z}$. Every signing query with label $\tau$ is simulated by setting $M_{\tau}=g^{-c_{\tau, 0}}$ and $S_{\tau}=\left(g_{1}^{c_{\tau, 1}}\right)$. The signature is correctly distributed since $(1) S_{\tau}=\left(\mathrm{H}(\tau) \cdot M_{\tau}\right)^{1 / z}$, and $(2) M_{\tau}$ looks random thanks to the programmable pseudo-randomness of $\mathrm{H}$. To conclude the proof, assume that the adversary comes up with a forgery $M^{*}, S^{*}$ for label $\tau^{*}$ such that $\tau^{*}$ was already queried, and let $\hat{S}, \hat{M}$ be the values in the simulation of the signing query for $\tau^{*}$. Now, $\hat{S}=\left(\mathrm{H}\left(\tau^{*}\right) \cdot \hat{M}\right)^{1 / z}$ holds by correctness, while $S^{*}=\left(\mathrm{H}\left(\tau^{*}\right) \cdot M^{*}\right)^{1 / z}$ holds for $M^{*} \neq \hat{M}$ by definition of forgery. Then $\left(M^{*} / \hat{M}, S^{*} / \hat{S}\right)$ is clearly a solution to the above assumption. This essentially shows that we can sign as many $M$ 's as the number of $\tau$ 's, that is $N$. And by using our construction $\mathrm{H}=\mathrm{H}_{\text {sqrt }}$ this is achievable with a key of length $O(\sqrt{N})$. Let us stress that the above one is an incomplete proof sketch, that we give only to illustrate the core ideas of using programmable pseudo-randomness. Moreover, note that the one presented above is only one of the possible cases of a forgery, but we think that it is the most interesting one to be considered in our example. We defer the reader to Section 3.4 for a precise description of our signature scheme and its security proof.

\subsubsection{Related Work}

Hanaoka, Matsuda and Schuldt [80] show that there cannot be any black-box construction of a (poly, 1)-PHF. The latter result has been overcome by the recent work of Freire et al. 64] who propose a (poly, 1)-PHF based on multilinear maps slightly changing the definition of PHFs in order to work in the multilinear group setting. Their (poly, 1)-PHF leads to several applications, notably standard-model versions (over multilinear groups) of BLS signatures, the BonehFranklin IBE, and identity-based non-interactive key-exchange. While the notion 
of PHFs in the multilinear setting of [64] is different from our APHFs (with the main difference being that ours are secretly computable), it is worth noting that the two notions have some relation. As we discuss in Section 3.3.1, our APHFs indeed imply PHFs in the bilinear setting (though carrying the same degree of programmability).

The idea of using bilinear maps to reduce the size of public keys was used previously by Haralambiev et al. [81] in the context of public-key encryption, and by Yamada et al. [116] in the context of digital signatures. We note that our solutions use a similar approach in the construction of APHFs, which however also include the important novelty of programmable pseudorandomness, that turned out to be crucial in our proofs for the linearly-homomorphic signature.

Our work is also related to the research line on Linearly-Homomorphic Structure Preserving Signatures (LHSPS). Structure preserving signatures (SPSs) are a particular kind of cryptographic signatures in which messages, public key elements and signatures are all elements of a group over which there exists an efficiently computable bilinear map. In [92], Libert et al. introduced structure preserving signatures with linearly homomorphic properties: a bit more in detail, these signature schemes act exactly as other linearly homomorphic signatures, with the additional restriction that, as in SPSs, signatures and messages are vectors of group elements and the linearly homomorphic property holds with respect to the group operation. Moreover, the model adopted by works on LHSPSs is slightly different from the one of linearly-homomorphic signatures (as defined in [31, 63] and used in this paper). In LHSPSs one signs vectors along with a vector identifier, and security is defined so that an output by an adversary is considered a forgery if it consists of a valid signature on a vector which does not belong to the linear span of originally signed vectors. It is known that a LHSPS (following this model) could be used to construct a scheme that signs messages (which can also be vectors) in a dataset, as in the model considered by this paper: given a vector $\vec{v}_{i}$ at position $i$, one uses the LHSPS to sign the vector of group elements $g^{\vec{u}_{i}}$ where $\vec{u}_{i}=\vec{e}_{i} \| \vec{v}_{i}$, with $\vec{e}_{i}$ the $i$-th column of the identity matrix. However, since in all existing LHSPSs (in the standard model) the size of the public key is linear in the dimension of the signed vectors, using LHSPSs yields solutions with a public key linear in the dataset size. On the other hand, it remains an open problem to design a LHSPS whose public key is sub-linear in the dimension of the signed vectors. Indeed, our techniques for reducing the public key size do not seem to work in the structure-preserving setting due to the fact that messages are group elements whose discrete logarithms are not known.

\subsection{Preliminaries}

In this section, we review some basic definitions that we use in our work. 
Chapter 3. Improving Efficiency: Linearly Homomorphic Signatures with Sublinear Public Keys via APHFs

Bilinear Groups and Complexity Assumptions. Let $\lambda \in \mathbb{N}$ be a security parameter and let $\mathcal{G}\left(1^{\lambda}\right)$ be an algorithm which takes as input the security parameter and outputs the description of (asymmetric) bilinear groups bgp $=\left(p, \mathbb{G}_{1}, \mathbb{G}_{2}, \mathbb{G}_{T}, e, g_{1}, g_{2}\right)$ where $\mathbb{G}_{1}, \mathbb{G}_{2}$ and $\mathbb{G}_{T}$ are groups of the same prime order $p>2^{\lambda}, g_{1} \in \mathbb{G}_{1}$ and $g_{2} \in \mathbb{G}_{2}$ are two generators, and $e: \mathbb{G}_{1} \times \mathbb{G}_{2} \rightarrow \mathbb{G}_{T}$ is an efficiently computable, non-degenerate, bilinear map, and there is no efficiently computable isomorphism between $\mathbb{G}_{1}$ and $\mathbb{G}_{2}$. We call such an algorithm $\mathcal{G}$ a bilinear group generator. In the case $\mathbb{G}_{1}=\mathbb{G}_{2}$, the groups are said symmetric, else they are said asymmetric.

In our work we rely on specific computational and decisional assumptions in such bilinear groups.

Definition 8 ( $q$-Strong Diffie-Hellman [28]). Let $\mathcal{G}$ be a generator of asymmetric bilinear groups, let bgp $=\left(p, \mathbb{G}_{1}, \mathbb{G}_{2}, \mathbb{G}_{T}, g_{1}, g_{2}, e\right) \stackrel{\$}{\leftarrow}\left(1^{\lambda}\right)$ where $g_{1}, g_{2}$ are two random generators, and let $q=\operatorname{poly}(\lambda)$. We say that the $q$-Strong Diffie-Hellman Assumption ( $q-S D H)$ is $\epsilon$-hard for $\mathcal{G}$ if, for every PPT adversary $\mathcal{A}$,

$$
\mathbf{A d v}_{\mathcal{A}}^{q-S D H}(\lambda)=\operatorname{Pr}\left[\mathcal{A}\left(g_{1}, g_{1}^{z}, \ldots, g_{1}^{z^{q}}, g_{2}, g_{2}^{z}\right)=\left(c, g_{1}^{1 /(z+c)}\right) \mid z \stackrel{\$}{\leftarrow} \mathbb{Z}_{p}\right] \leq \epsilon
$$

Definition 9 ( $q$-Diffie-Hellman Inversion [27, 99]). Let $\mathcal{G}$ be a generator of asymmetric bilinear groups, let bgp $=\left(p, \mathbb{G}_{1}, \mathbb{G}_{2}, \mathbb{G}_{T}, g_{1}, g_{2}, e\right) \stackrel{\$}{\leftarrow} \mathcal{G}\left(1^{\lambda}\right)$ where $g_{1}, g_{2}$ are two random generators, and let $q=$ poly $(\lambda)$. We say that the $q$-Diffie-Hellman Inversion Assumption $(q-D H I)$ is $\epsilon$-hard for $\mathcal{G}$ if, for every PPT adversary $\mathcal{A}$,

$$
\mathbf{A d v}_{\mathcal{A}}^{q-D H I}(\lambda)=\operatorname{Pr}\left[\mathcal{A}\left(g_{1}, g_{1}^{z}, g_{2}^{z}, \ldots, g_{1}^{z^{q}}, g_{2}^{z^{q}}\right)=g_{1}^{1 / z} \mid z \stackrel{\$}{\leftarrow} \mathbb{Z}_{p}\right] \leq \epsilon
$$

It is not hard to see that the above problem is equivalent to the one in which the adversary is given the same input and is challenged to compute the "next power" $g_{1}^{z^{q+1}}$.

A weaker variant of the $q$-DHI assumption that we use in some of our proofs is the one in which the adversary receives only $g_{2}, g_{2}^{z}$ in the group $\mathbb{G}_{2}$. For coherence with [82] we call this assumption $q$-Diffie-Hellman $(q-\mathrm{DH})$.

Definition 10 (External Decisional Diffie-Hellman in $\mathbb{G}_{1}$ ). Let $\mathcal{G}$ be a generator of asymmetric bilinear groups, and let bgp $=\left(p, \mathbb{G}_{1}, \mathbb{G}_{2}, \mathbb{G}_{T}, g_{1}, g_{2}, e\right) \stackrel{\$}{\leftarrow} \mathcal{G}\left(1^{\lambda}\right)$. We say that the External Decisional Diffie-Hellman Assumption (XDDH) is $\epsilon$ hard in $\mathbb{G}_{1}$ if, for every PPT adversary $\mathcal{A}$, it holds

$$
\left|\operatorname{Pr}\left[\mathcal{A}\left(g_{1}, g_{1}^{a}, g_{1}^{b}, g_{1}^{a b}\right)=1 \mid a, b \stackrel{\$}{\leftarrow} \mathbb{Z}_{p}\right]-\operatorname{Pr}\left[\mathcal{A}\left(g_{1}, g_{1}^{a}, g_{1}^{b}, g_{1}^{c}\right)=1 \mid a, b, c \stackrel{\$}{\leftarrow} \mathbb{Z}_{p}\right]\right| \leq \epsilon
$$

Finally, we introduce the following static assumption over asymmetric bilinear groups, that we call "Flexible Diffie-Hellman Inversion" (FDHI) for its similarity to Flexible Diffie-Hellman [79]. FDHI is hard in the generic bilinear group model. 
Chapter 3. Improving Efficiency: Linearly Homomorphic Signatures with Sublinear Public Keys via APHFs

Definition 11 (Flexible Diffie-Hellman Inversion Assumption). Let $\mathcal{G}$ be a generator of asymmetric bilinear groups, and let bgp $=\left(p, \mathbb{G}_{1}, \mathbb{G}_{2}, \mathbb{G}_{T}, g_{1}, g_{2}, e\right) \stackrel{\$}{\leftarrow}$ $\mathcal{G}\left(1^{\lambda}\right)$. We say that the Flexible Diffie-Hellman Inversion (FDHI) Assumption is $\epsilon$-hard for $\mathcal{G}$ if for every PPT adversary $\mathcal{A}$ :

$$
\begin{gathered}
\operatorname{Adv}_{\mathcal{A}}^{F D H I}(\lambda)=\operatorname{Pr}\left[W \in \mathbb{G}_{1} \backslash\left\{1_{\mathbb{G}_{1}}\right\} \wedge W^{\prime}=W^{\frac{1}{z}}:\left(W, W^{\prime}\right) \leftarrow \mathcal{A}\left(g_{1}, g_{2}, g_{2}^{z}, g_{2}^{v}, g_{1}^{\frac{z}{v}},\right.\right. \\
\left.\left.g_{1}^{r}, g_{1}^{\frac{\underline{v}}{v}}\right) \mid z, r, v \stackrel{\$}{\leftarrow} \mathbb{Z}_{p}\right] \leq \epsilon
\end{gathered}
$$

\subsection{Asymmetric Programmable Hash Functions}

In this section we present our new notion of asymmetric programmable hash functions.

Let bgp $=\left(p, \mathbb{G}_{1}, \mathbb{G}_{2}, \mathbb{G}_{T}, g_{1}, g_{2}, e\right)$ be a family of asymmetric bilinear groups induced by a bilinear group generator $\mathcal{G}\left(1^{\lambda}\right)$ for a security parameter $\left.\lambda \in \mathbb{N}\right|^{4}$ An asymmetric group hash function $\mathrm{H}: \mathcal{X} \rightarrow \mathbb{G}_{1}$ consists of three PPT algorithms (H.Gen, H.PriEval, H.PubEval) working as follows:

H.Gen $\left(1^{\lambda}\right.$, bgp $) \rightarrow($ sek, pek $): \quad$ on input the security parameter $\lambda \in \mathbb{N}$ and a bilinear group description bgp, the PPT key generation algorithm outputs a (secret) evaluation key sek and a (public) evaluation key pek.

H.PriEval(sek, $X) \rightarrow Y \in \mathbb{G}_{1}$ : given the secret evaluation key sek and an input $X \in \mathcal{X}$, the deterministic evaluation algorithm returns an output $Y=$ $\mathrm{H}(X) \in \mathbb{G}_{1}$.

H.PubEval(pek, $X) \rightarrow \hat{Y} \in \mathbb{G}_{T}$ : on input the public evaluation key pek and an input $X \in \mathcal{X}$, the public evaluation algorithm outputs a value $\hat{Y} \in \mathbb{G}_{T}$ such that $\hat{Y}=e\left(\mathbf{H}(X), g_{2}\right)$.

For asymmetric hash functions satisfying the syntax described above, we define two different properties that model their possible programmability.

The first property is a generalization of the notion of programmable hash functions of [83, 84] to our asymmetric setting (i.e., where the function is only secretly-computatble), and to the more specific setting of bilinear groups. The basic idea is that it is possible to generate the function in a trapdoor-mode that allows one to express every output of $\mathrm{H}$ in relation to some specified group elements. In particular, the most useful fact of programmability is that for two arbitrary disjoint sets of inputs $\bar{X}, \bar{Z} \subset \mathcal{X}$, the joint probability that some of these group elements appear in $\mathrm{H}(Z), \forall Z \in \bar{Z}$ and do not appear in $\mathrm{H}(X), \forall X \in \bar{X}$ is significant.

\footnotetext{
${ }^{4}$ Our definition can be easily adapted to work in symmetric bilinear groups where $\mathbb{G}_{1}=\mathbb{G}_{2}$.
} 
Definition 12 (Asymmetric Programmable Hash Functions). We say that an asymmetric group hash function $\mathrm{H}=(\mathrm{H}$. Gen, H.PriEval, H.PubEval) is $(m, n, d, \gamma, \delta)$ -programmable if there exist an efficient trapdoor generation algorithm $\mathrm{H}$. TrapGen and an efficient trapdoor evaluation algorithm $\mathrm{H}$.TrapEval such that:

Syntax: H.TrapGen $\left(1^{\lambda}\right.$, bgp, $\left.\hat{g}_{1}, \hat{h}_{1}, \hat{g}_{2}, \hat{h}_{2}\right) \rightarrow(\mathrm{td}$, pek) takes as input the security parameter $\lambda$, bilinear group description bgp and group elements $\hat{g}_{1}, \hat{h}_{1} \in$ $\mathbb{G}_{1}, \hat{g}_{2}, \hat{h}_{2} \in \mathbb{G}_{2}$, and it generates a public hash key pek along with a trapdoor td. H.TrapEval $(\mathrm{td}, X) \rightarrow \vec{c}_{X}$ takes as input the trapdoor information $\mathrm{td}$ and an input $X \in \mathcal{X}$, and outputs a vector of integer coefficients $\vec{c}_{X}=$ $\left(c_{0}, \ldots, c_{d^{\prime}}\right) \in \mathbb{Z}^{d^{\prime}}$ of a 2-variate polynomial $c_{X}\left(y_{1}, y_{2}\right)$ of degree $\leq d$.

Correctness: For all group elements $\hat{g}_{1}, \hat{h}_{1} \in \mathbb{G}_{1}, \hat{g}_{2}, \hat{h}_{2} \in \mathbb{G}_{2}$ such that $\hat{h}_{1}=\hat{g}_{1}^{y_{1}}$ and $\hat{h}_{2}=\hat{g}_{2}^{y_{2}}$ for some $y_{1}, y_{2} \in \mathbb{Z}_{p}$, for all trapdoor keys (td, pek) $\stackrel{\$}{\leftarrow}$ H.TrapGen $\left(1^{\lambda}, \hat{g}_{1}, \hat{h}_{1}, \hat{g}_{2}, \hat{h}_{2}\right)$, and for all inputs $X \in \mathcal{X}$, if $\vec{c}_{X} \leftarrow \mathrm{H}$.TrapEval(td, $X)$, then

$$
\mathrm{H}(X)=\hat{g}_{1}^{c_{X}\left(y_{1}, y_{2}\right)}
$$

Statistically-close trapdoor keys: For all generators $\hat{g}_{1}, \hat{h}_{1} \in \mathbb{G}_{1}, \hat{g}_{2}, \hat{h}_{2} \in \mathbb{G}_{2}$ and for all (sek, pek) $\stackrel{\$}{\leftarrow} \mathrm{H} . \mathrm{Gen}\left(1^{\lambda}\right),\left(\mathrm{td}\right.$, pek $\left.^{\prime}\right) \stackrel{\$}{\leftarrow} \mathrm{H}$.TrapGen $\left(1^{\lambda}, \hat{g}_{1}, \hat{h}_{1}, \hat{g}_{2}\right.$, $\left.\hat{h}_{2}\right)$, the distribution of the public keys pek and pek' $^{\prime}$ is within statistical distance $\gamma$.

Well distributed logarithms: For all $\hat{g}_{1}, \hat{h}_{1} \in \mathbb{G}_{1}, \hat{g}_{2}, \hat{h}_{2} \in \mathbb{G}_{2}$, all keys (td, pek) $\stackrel{\$}{\leftarrow}$.TrapGen $\left(1^{\lambda}, \hat{g}_{1}, \hat{h}_{1}, \hat{g}_{2}, \hat{h}_{2}\right)$, and all inputs $X_{1}, \ldots, X_{m} \in \mathcal{X}$ and $Z_{1}, \ldots$, $Z_{n} \in \mathcal{X}$ such that $X_{i} \neq Z_{j}$ for all $i, j$, we have

$$
\operatorname{Pr}\left[c_{X_{1}, 0}=\cdots=c_{X_{m}, 0}=0 \wedge c_{Z_{1}, 0}, \ldots, c_{Z_{n}, 0} \neq 0\right] \geq \delta
$$

where $\vec{c}_{X_{i}} \leftarrow \mathrm{H}$. TrapEval(td, $\left.X_{i}\right)$ and $\vec{c}_{Z_{j}} \leftarrow \mathrm{H}$. TrapEval(td, $Z_{j}$ ), and $c_{X_{i}, 0}$ (resp. $\left.c_{Z_{j}, 0}\right)$ is the coefficient of the term of degree 0 . The probability is over the trapdoor $\mathrm{td}$ that was produced along with pek.

If $\gamma$ is negligible and $\delta$ is noticeable we simply say that $\mathrm{H}$ is $(m, n, d)$-programmable. Furthermore, if $m$ (resp. $n$ ) is an arbitrary polynomial in $\lambda$, then we say that $\mathrm{H}$ is (poly, $n, d)$-programmable (resp. ( $m$, poly, $d$ )-programmable). Finally, if $\mathrm{H}$ admits trapdoor algorithms that satisfy only the first three properties, then $\mathrm{H}$ is said simply $(d, \gamma)$-programmable. Note that any $\mathrm{H}$ that is $(m, n, d, \gamma, \delta)$-programmable is also $(d, \gamma)$-programmable.

Programmable Pseudorandomness. The second main programmability property that we define for asymmetric hash functions is quite different from the previous one. It is called programmable pseudorandomness, and very intuitively it says that, when using the hash function in trapdoor mode, it is possible 
Chapter 3. Improving Efficiency: Linearly Homomorphic Signatures with Sublinear Public Keys via APHFs

to "embed" a PRF into it. More precisely, the trapdoor algorithms satisfy programmable pseudorandomness if they allow to generate keys such that even by observing pek and $\mathrm{H}(X)$ for a bunch of inputs $X$, then the elements $g_{1}^{c_{X, 0}}$ look random. The formal definition follows:

Definition 13 (Asymmetric Hash Functions with Programmable Pseudorandomness). An asymmetric hash function $\mathrm{H}=(\mathrm{H} . G e n$, H.PriEval, H.PubEval) has $(d, \gamma, \epsilon)$-programmable pseudorandomness if there exist efficient trapdoor algorithms H.TrapGen, H.TrapEval that satisfy the properties of syntax, correctness, and $\gamma$-statistically-close trapdoor keys as in Definition 12, and additionally satisfy the following property with parameter $\epsilon$ :

Pseudorandomness: Let $b \in\{0,1\}$ and let $\operatorname{Exp}_{\mathcal{A}, \mathrm{H}}^{P R H-b}(\lambda)$ be the following experiment between an adversary $\mathcal{A}$ and a challenger.

1. Generate bgp $\stackrel{\$}{\leftarrow} \mathcal{G}\left(1^{\lambda}\right)$, and run $\mathcal{A}$ (bgp), that outputs two generators $h_{1} \in \mathbb{G}_{1}, h_{2} \in \mathbb{G}_{2}$.

2. Compute (td, pek) $\stackrel{\$}{\leftarrow} \mathrm{H}$. TrapGen $\left(1^{\lambda}, g_{1}, h_{1}, g_{2}, h_{2}\right)$ and run $\mathcal{A}($ pek $)$ with access to the following oracle:

- If $b=0, \mathcal{A}$ is given $\mathcal{O}(\cdot)$ that on input $X \in \mathcal{X}$ returns $\mathrm{H}(X)=$ $g_{1}^{c_{X}\left(y_{1}, y_{2}\right)}$ and $g_{1}^{c_{X, 0}}$, where $\vec{c}_{X} \leftarrow \mathrm{H}$.TrapEval $(\mathrm{td}, X)$;

- If $b=1, \mathcal{A}$ is given $\mathcal{R}(\cdot)$ that on input $X \in \mathcal{X}$ returns $\mathrm{H}(X)=$ $g_{1}^{c_{X}\left(y_{1}, y_{2}\right)}$ and $g_{1}^{r_{X}}$, for a randomly chosen $r_{X} \stackrel{\$}{\leftarrow} \mathbb{Z}_{p}$ (which is unique for every $X \in \mathcal{X}$ ).

3. At the end the adversary outputs a bit $b^{\prime}$, and $b^{\prime}$ is returned as the output of the experiment.

Then we say that $\mathrm{H}$.TrapGen, $\mathrm{H}$.TrapEval satisfy pseudorandomness for $\epsilon$, if for all PPT $\mathcal{A}$

$$
\left|\operatorname{Pr}\left[\operatorname{Exp}_{\mathcal{A}, \mathrm{H}}^{P R H-0}(\lambda)=1\right]-\operatorname{Pr}\left[\operatorname{Exp}_{\mathcal{A}, \mathrm{H}}^{P R H-1}(\lambda)=1\right]\right| \leq \epsilon
$$

where the probabilities are taken over all the random choices of TrapGen, the oracle $\mathcal{R}$ and the adversary $\mathcal{A}$.

Remark 2 (On the mutual existence of programmability and programmable pseudorandomness). We stress that the two properties of programmability and programmable pseudorandomness defined above are mutually exclusive. Precisely, an APHF can have a pair of trapdoor algorithms (TrapGen, TrapEval) that admits either $(m, n, d, \gamma, \delta)$-programmability (for non-negligible $\delta$ ), or $(d, \gamma, \epsilon)$-programmable pseudorandomness (for negligible $\epsilon$ ). Intuitively, the reason why the same trapdoor algorithms cannot satisfy both properties is that $(m, n, \delta, \gamma)$-programmability implies that for any elements $X_{1}, \ldots, X_{m} \in \mathcal{X}$ it holds $c_{X_{i, 0}}=0$ with non negligible probability $\delta$. However, if this holds then programmable pseudorandomness can be trivially broken, since $g_{1}^{c^{c}, 0}=1$ with non negligible probability $\delta$. 
Chapter 3. Improving Efficiency: Linearly Homomorphic Signatures with Sublinear Public Keys via APHFs

On the other hand, it is quite interesting to observe that the same function can enjoy both properties through different, appropriate, pairs of trapdoor algorithms. In fact, an asymmetric group hash function can have a pair of trapdoor algorithms (TrapGen, TrapEval) for which $(m, n, \delta, \gamma)$-programmability holds, and another pair of trapdoor algorithms (TrapGen', TrapEval') for which $(d, \gamma, \delta)$ programmable pseudorandomness holds. Then, since all trapdoor generations produce keys that are statistically indistinguishable from the real ones it follows that also the two trapdoor modes are statistically indistinguishable. In a nutshell, this means that the same function can be programmed in different modes in different steps of a security proof, a property which turns out to be very useful, for example, in our proofs of Section 3.4 .3 .

Other variants of programmability. Here we define two other variants of the programmability notion given in Definition 12 .

Weak Programmability. We consider a weak version of the above programmability property in which one fixes at key generation time the $n$ inputs $Z_{j}$ on which $c_{Z_{j}, 0} \neq 0$.

Definition 14 (Asymmetric Weakly-Programmable Hash Functions). An asymmetric group hash function $\mathrm{H}=(\mathrm{H} . G e n, H . P r i E v a l, H . P u b E v a l)$ is weakly $(m, n$, $d, \gamma, \delta)$-programmable if there exist efficient trapdoor generation $\mathrm{H}$.TrapGen and trapdoor evaluation $\mathrm{H}$. TrapEval algorithms such that:

- Syntax: H.TrapGen $\left(1^{\lambda}\right.$, bgp, $\left.\hat{g}_{1}, \hat{h}_{1}, \hat{g}_{2}, \hat{h}_{2}, Z_{1}, \ldots, Z_{n}\right) \rightarrow$ (td, pek) takes as input the security parameter $\lambda$, bilinear group description bgp, group elements $\hat{g}_{1}, \hat{h}_{1} \in \mathbb{G}_{1}, \hat{g}_{2}, \hat{h}_{2} \in \mathbb{G}_{2}$, and a set of $n$ inputs $Z_{1}, \ldots, Z_{n} \in \mathcal{X}$. It generates a public hash key pek along with a trapdoor $\mathrm{td}$. H.TrapEval $(\mathrm{td}, X) \rightarrow$ $\vec{c}_{X}$ works exactly as in Definition 12.

- The properties of correctness and statistically-close trapdoor keys hold as in Definition 12. The property of well-distributed logarithms is also the same except that the inputs $Z_{1}, \ldots, Z_{n}$ are the ones fixed as input to $\mathrm{H}$.TrapGen.

Degree- $d$ Programmability. In our work we also consider a variant of the above definition in which the property of well distributed logarithms is stated with respect to the degree-d coefficients of the polynomials generated by $\mathrm{H}$.TrapEval. In this case, we say that $\mathrm{H}$ is $(m, n, d, \gamma, \delta)$-degree-d-programmable.

\subsubsection{Relation with existing notions}

Before describing our realizations of APHFs, we discuss here the relation between our new notion and two existing notions of programmable hash functions:

the original one by Hofheinz and Kiltz [83] and its adaptation to the multilinear setting recently proposed by Freire et al. 64]. 
Chapter 3. Improving Efficiency: Linearly Homomorphic Signatures with Sublinear Public Keys via APHFs

When working over bilinear groups, the notion of programmable hash functions of [83] is essentially a special case of ours. The main differences are: (1) PHFs are publicly computable, (2) the trapdoor algorithms work with only two generators $\hat{g}, \hat{h}$ and every output of the function can be expressed as a linear function $\hat{g}^{a} \hat{h}^{b}$ of these two generators. As we formally state in the following theorem, a standard PHF is an APHF for $d=1$ :

Theorem 4. Let $\hat{\mathrm{H}}=$ (PHF.Gen, PHF.Eval) be an $(m, n, \gamma, \delta)$-programmable hash function such that $\hat{\mathrm{H}}: \mathcal{X} \rightarrow \mathbb{G}_{1}$. Define H.Gen = PHF.Gen, H.PriEval = PHF.Eval and (informally) H.PubEval $=e\left(\right.$ PHF.Eval, $\left.g_{2}\right)$. Then $\mathrm{H}=(\mathrm{H} . G e n$, H.PriEval, H.PubEval) is an asymmetric $(m, n, 1, \gamma, \delta)$-programmable hash function.

The proof is straightforward and is omitted.

Second, we analyze the relation between asymmetric hash functions and the PHFs in the multilinear setting introduced in [64]. Informally, for a setting of leveled multilinear groups $\mathbb{G}_{1}, \ldots, \mathbb{G}_{\ell}, 64$ considers a group hash function $\hat{\mathrm{H}}$ : $\mathcal{X} \rightarrow \mathbb{G}_{\ell}$. Then, $\hat{\mathrm{H}}$ is said $(m, n)$-programmable if there exist two trapdoor algorithms PHF.TrapGen, PHF.TrapEval such that: PHF.TrapGen $\left(1^{\lambda}, g_{1}, \ldots, g_{\ell}\right.$, $h$ ) takes as input $g_{i}, h \in \mathbb{G}_{1}$ with $h \neq 1$ and outputs a trapdoor td and hash key hk; PHF.TrapEval(td, $X)$ on input $X$ outputs an integer $a_{X}$ and an element $B_{X} \in \mathbb{G}_{\ell-1}$ such that $\mathrm{H}(X)=e\left(g_{1}, \ldots, g_{\ell}\right)^{a_{X}} e\left(B_{X}, h\right) \in \mathbb{G}_{\ell}$. If we consider leveled bilinear groups where $\mathbb{G}=\mathbb{G}_{1}$ and $\mathbb{G}_{T}=\mathbb{G}_{2}$, then asymmetric programmable hash functions (for $d \leq 2$ ) imply PHFs in the (symmetric) bilinear group setting:

Theorem 5. Let $\mathbb{G}, \mathbb{G}_{T}$ be symmetric bilinear groups, and let $\mathrm{H}=(\mathrm{H} . \mathrm{Gen}$, H.PriEval, H.PubEval) be an asymmetric $(m, n, 2, \gamma, \delta)$-programmable hash function such that $\mathrm{H}: \mathcal{X} \rightarrow \mathbb{G}$. Define PHF.Gen $=$ H.Gen and PHF.Eval $=$ H.PubEval. Then $\hat{\mathrm{H}}=$ (PHF.Gen, PHF.Eval) is an $(m, n, \gamma, \delta)$-programmable hash function in the bilinear setting.

The proof is fairly easy. Here we provide a sketch. Basically, by assuming that $\mathrm{H}$ is programmable, we have to show two algorithms PHF.TrapGen, PHF. TrapEval that satisfy the programmability of $\hat{\mathrm{H}}$ in the bilinear setting:

PHF.TrapGen $\left(1^{\lambda}, g, h\right)$ : run (td, pek) $\stackrel{\$}{\leftarrow}$ H.TrapGen $\left(1^{\lambda}, g, h\right)$ and output (td, pek).

PHF.TrapEval $(\mathrm{td}, X):$ run $\vec{c}_{X} \leftarrow \mathrm{H}$.TrapEval(td, $\left.X\right)$ to generate the coefficient of a degree-2 polynomial $c_{X}(y)$ where $y=D \log _{g}(h)$. Then output $a_{X}=c_{X, 0}$, and $B_{X}=g^{c_{X, 1}} h^{c_{X, 2}}$.

It is easy to see that if $\vec{c}_{X}$ is such that $\mathrm{H}(X)=g^{c_{X, 0}+c_{X, 1} y+c_{X, 2} y^{2}}$ then

$$
\hat{\mathbf{H}}(X)=e(\mathbf{H}(X), g)=e(g, g)^{c_{X, 0}} e\left(g^{c_{X, 1}+c_{X, 2} y}, g^{y}\right)=e(g, g)^{a_{X}} e\left(B_{X}, h\right)
$$

Finally, the $(m, n, \gamma, \delta)$-programmability of $\hat{\mathrm{H}}$ is immediately implied by the well distribution of the discrete logarithms in $\mathbf{H}$ for parameters $(m, n, 2, \gamma, \delta)$. 
Chapter 3. Improving Efficiency: Linearly Homomorphic Signatures with Sublinear Public Keys via APHFs

\subsubsection{An Asymmetric Programmable Hash Function based on Cover-Free Sets}

In this section we present the construction of an asymmetric hash function, $\mathrm{H}_{\text {acfs }}$, based on cover-free sets. Our construction uses ideas similar to the ones used by Hofheinz, Jager and Kiltz [82] to design a (regular) programmable hash function. Our construction extends these ideas with a technique that allows us to obtain a much shorter public key. Concretely, for binary inputs of size $\ell$, the programmable hash function $\mathrm{H}_{\mathrm{cfs}}$ in 82 is $(m, 1)$-programmable with a hash key of length $O\left(\ell \mathrm{m}^{2}\right)$. In contrast, our new construction $\mathrm{H}_{\text {acfs }}$ is $(m, 1)$-programmable with a hash key of length $O(m \sqrt{\ell})$. While such improvement is obtained at the price of obtaining the function in the secret-key model, our results of Section 3.5 show that asymmetric programmable hash are still useful to build short bilinearmap signatures, whose efficiency, in terms of signature's and key's length matches that of state-of-the-art schemes [116].

Before proceeding with describing our function, below we recall the notion of cover-free sets.

Cover-Free Families. If $S, V$ are sets, we say that $S$ does not cover $V$ if $S \nsupseteq V$. Let $T, m, s$ be positive integers, and let $F=\left\{F_{i}\right\}_{i \in[s]}$ be a family of subsets of $[T]$. A family $F$ is said to be $m$-cover-free over $[T]$, if for any subset $I \subseteq[s]$ of cardinality at most $m$, then the union $\cup_{i \in I} F_{i}$ does not cover $F_{j}$ for all $j \notin I$. More formally, for any $I \subseteq[s]$ such that $|I| \leq m$, and any $j \notin I, \cup_{i \in I} F_{i} \nsupseteq F_{j}$. Furthermore, we say that $F$ is $w$-uniform if every subset $F_{i}$ in the family have size $w$. In our construction, we use the following fact from [60, 89]:

Lemma 7 ([60, 89]). There is a deterministic polynomial time algorithm that, on input integers $s=2^{\ell}$ and $m$, returns $w, T, F$ where $F=\left\{F_{i}\right\}_{i \in[s]}$ is a w-uniform, $m$-cover-free family over $[T]$, for $w=T / 4 m$ and $T \leq 16 m^{2} \ell$.

The Construction of $\mathrm{H}_{\mathrm{acfs}}$. Let $\mathcal{G}\left(1^{\lambda}\right)$ be a bilinear group generator, let bgp $=$ $\left(p, \mathbb{G}_{1}, \mathbb{G}_{2}, \mathbb{G}_{T}, g_{1}, g_{2}, e\right)$ be an instance of bilinear group parameters generated by $\mathcal{G}$. Let $\ell=\ell(\lambda)$ and $m=m(\lambda)$ be two polynomials in the security parameter. We set $s=2^{\ell}, T=16 m^{2} \ell$, and $w=T / 4 m$ as for Lemma 7, and define $t=\lceil\sqrt{T}\rceil$. Note that every integer $k \in[T]$ can be written as a pair of integers $(i, j) \in[t] \times[t]$ using some canonical mapping. For the sake of simplicity, sometimes we abuse notation and write $(i, j) \in[T]$ where $i, j \in[t]$.

In the following we describe the asymmetric hash function $\mathrm{H}_{\mathrm{acfs}}=(\mathrm{H} . \mathrm{Gen}$, H.PriEval, H.PubEval) that maps $\mathrm{H}_{\text {acfs }}: \mathcal{X} \rightarrow \mathbb{G}_{1}$ where $\mathcal{X}=\{0,1\}^{\ell}$. In particular, every input $X \in\{0,1\}^{\ell}$ is associated to a set $F_{i}, i \in\left[2^{\ell}\right]$, by interpreting $X$ as an integer in $\left\{0, \ldots, 2^{\ell}-1\right\}$ and by setting $i=X+1$. We call $F_{X}$ such subset associated to $X$.

H.Gen $\left(1^{\lambda}\right.$, bgp $):$ for $i=1$ to $t$, sample $\alpha_{i}, \beta_{i} \stackrel{\$}{\leftarrow} \mathbb{Z}_{p}$ and compute $A_{i}=g_{1}^{\alpha_{i}}, B_{i}=$ $g_{2}^{\beta_{i}}$. Finally, set sek $=\left\{\alpha_{i}, \beta_{i}\right\}_{i=1}^{t}$, pek $=\left\{A_{i}, B_{i}\right\}_{i=1}^{t}$, and return (sek, pek). 
Chapter 3. Improving Efficiency: Linearly Homomorphic Signatures with Sublinear Public Keys via APHFs

H.PriEval(sek, $X)$ : first, compute the subset $F_{X} \subseteq[T]$ associated to $X \in\{0,1\}^{\ell}$, and then return

$$
Y=g_{1}^{\sum_{(i, j) \in F_{X}} \alpha_{i} \beta_{j}} \in \mathbb{G}_{1}
$$

H.PubEval(pek, $X)$ : let $F_{X} \subseteq[T]$ be the subset associated to $X$, and compute

$$
\hat{Y}=\prod_{(i, j) \in F_{X}} e\left(A_{i}, B_{j}\right)=e\left(\mathrm{H}(X), g_{2}\right)
$$

Theorem 6. Let $\mathcal{G}$ be a bilinear group generator. The hash function $\mathrm{H}_{\mathrm{acfs}}$ described above is an asymmetric $(m, n, d, \gamma, \delta)$-programmable hash function with $n=1, d=2, \gamma=0$ and $\delta=1 / T$.

Proof. First, we describe the trapdoor algorithms:

H.TrapGen $\left(1^{\lambda}\right.$, bgp, $\left.\hat{g}_{1}, \hat{h}_{1}, \hat{g}_{2}, \hat{h}_{2}\right)$ : first, sample $a_{i}, b_{i} \stackrel{\$}{\leftarrow} \mathbb{Z}_{p}$ for all $i \in[t]$, and pick a random index $\tau \stackrel{\$}{\leftarrow}[T]$. Parse $\tau=\left(i^{*}, j^{*}\right) \in[t] \times[t]$. Next, set $A_{i^{*}}=\hat{g}_{1} \hat{h}_{1}^{a_{i^{*}}}, B_{j^{*}}=\hat{g}_{2} \hat{h}_{2}^{b_{j^{*}}}, A_{i}=\hat{h}_{1}^{a_{i}}, \forall i \neq i^{*}$, and $B_{j}=\hat{h}_{2}^{b_{j}}, \forall j \neq j^{*}$. Finally, set $\mathrm{td}=\left(\tau,\left\{a_{i}, b_{i}\right\}_{i=1}^{t}\right)$, pek $=\left\{A_{i}, B_{i}\right\}_{i=1}^{t}$, and output (td, pek).

H.TrapEval(td, $X)$ : first, compute the subset $F_{X} \subseteq[T]$ associated to $X \in\{0,1\}^{\ell}$, and then return the coefficients of the degree- 2 polynomial

$$
c_{X}\left(y_{1}, y_{2}\right)=\sum_{(i, j) \in F_{X}} \alpha_{i}\left(y_{1}\right) \cdot \beta_{j}\left(y_{2}\right)
$$

where every $\alpha_{i}\left(y_{1}\right)$ (resp. $\left.\beta_{j}\left(y_{2}\right)\right)$ is the discrete logarithm of $A_{i}$ (resp. $B_{j}$ ) in base $\hat{g}_{1}$ (resp. $\hat{g}_{2}$ ), viewed as a degree-1 polynomial in the unknown $y_{1}$ (resp. $\left.y_{2}\right)$.

Now, we show that the two trapdoor algorithms described above satisfy the four properties of Definition 12. First, syntax and correctness immediately follow by construction. Second, observe that each element $A_{i}$ (resp. $B_{j}$ ) in pek is a uniformly distributed group element in $\mathbb{G}_{1}$ (resp. $\mathbb{G}_{2}$ ), exactly as in the output of H.Gen, hence $\gamma=0$. Third, we show that the algorithms allow for well distributed logarithms for the case $n=1$. Let $X_{1}, \ldots, X_{m}, Z \in \mathcal{X}$ such that $Z \neq X_{i}$ for all $i$. From the $m$-cover-free property of $F$ we have that there exist an index $\tau^{\prime} \in F_{Z}$ such that $\tau^{\prime} \notin \cup_{i=1}^{m} F_{X_{i}}$. Since $\tau$ is chosen uniformly at random in $[T]$, we have that $\tau=\tau^{\prime}$ with probability $\delta=1 / T$. Now, assume that $\tau^{\prime}=\tau=\left(i^{*}, j^{*}\right) \in$ $[t] \times[t]$. Then for all $(i, j) \neq\left(i^{*}, j^{*}\right)$ it holds that the degree-0 coefficient of $c\left(y_{1}, y_{2}\right)=\alpha_{i}\left(y_{1}\right) \beta_{j}\left(y_{2}\right)$ is $c_{0}=0$, whereas for $\left(i^{*}, j^{*}\right)$ the degree-0 coefficient of $c^{*}\left(y_{1}, y_{2}\right)=\alpha_{i^{*}}\left(y_{1}\right) \beta_{j^{*}}\left(y_{2}\right)=\left(a_{i^{*}} y_{1}+1\right)\left(b_{j^{*}} y_{2}+1\right)$, is $c_{0}^{*}=1$. Therefore, we have that $c_{X_{i}, 0}=0, \forall i \in[\mathrm{m}]$ and $c_{Z, 0}=1$ holds with probability $\delta$. 
Chapter 3. Improving Efficiency: Linearly Homomorphic Signatures with Sublinear Public Keys via APHFs

\subsubsection{An Asymmetric Programmable Hash Function with Small Domain}

In this section, we present the construction of an asymmetric hash function, $\mathrm{H}_{\text {sqrt }}$, whose domain is of polynomial size $T$. $\mathrm{H}_{\text {sqrt }}$ has a public key of length $O(\sqrt{T})$, and it turns out to be very important for obtaining our linearly-homomorphic signature scheme with short public key presented in Section 3.4. Somewhat interestingly, we show that this new function $\mathrm{H}_{\text {sqrt }}$ satisfies several programmability properties, that make it useful in the context of various security proofs.

Let $\mathcal{G}\left(1^{\lambda}\right)$ be a bilinear group generator, let $T=\operatorname{poly}(\lambda)$ and $t=\lceil\sqrt{T}\rceil$. The hash function $\mathrm{H}_{\text {sqrt }}=$ (H.Gen, H.PriEval, H.PubEval) that maps $\mathrm{H}_{\text {sqrt }}: \mathcal{X} \rightarrow \mathbb{G}_{1}$ with $\mathcal{X}=[T]$ is defined as follows.

H.Gen $\left(1^{\lambda}\right.$, bgp $):$ for $i=1$ to $t$, sample $\alpha_{i}, \beta_{i} \stackrel{\$}{\leftarrow} \mathbb{Z}_{p}$ and compute $A_{i}=g_{1}^{\alpha_{i}}, B_{i}=$ $g_{2}^{\beta_{i}}$. Finally, set sek $=\left\{\alpha_{i}, \beta_{i}\right\}_{i=1}^{t}$, pek $=\left\{A_{i}, B_{i}\right\}_{i=1}^{t}$, and return(sek, pek $)$.

H.PriEval(sek, $X)$ : first, write $X \in[T]$ as a pair of integer $(i, j) \in[t] \times[t]$, and then return

$$
Y=g_{1}^{\alpha_{i} \beta_{j}} \in \mathbb{G}_{1}
$$

H.PubEval(pek, $X)$ : let $X=(i, j)$. The public evaluation algorithm returns

$$
\hat{Y}=e\left(A_{i}, B_{j}\right)=e\left(\mathbf{H}(X), g_{2}\right)
$$

Programmable Pseudorandomness of $\mathrm{H}_{\text {sqrt }}$. Here we show that $\mathrm{H}_{\text {sqrt }}$ satisfies the programmable pseudorandomness property of Definition 13 .

Theorem 7. Let $\mathbb{G}_{1}$ be a bilinear group of order $p$ over which the XDDH assumption is $\epsilon^{\prime}$-hard. Then the asymmetric hash function $\mathrm{H}_{\text {sqrt }}$ described above satisfies $(2,0, \epsilon)$-programmable pseudorandomness with $\epsilon=T \cdot \epsilon^{\prime}$. Furthermore, in the case when $h_{1}=1 \in \mathbb{G}_{1}$ or $h_{1}=g_{1}, \mathrm{H}_{\text {sqrt }}$ has $(1,0, \epsilon)$-programmable pseudorandomness.

Proof. First, we describe the trapdoor algorithms:

H.TrapGen $\left(1^{\lambda}, g_{1}, h_{1}, g_{2}, h_{2}\right)$ : first, sample $a_{i}, r_{i}, s_{i}, b_{i} \stackrel{\$}{\leftarrow} \mathbb{Z}_{p}$ for all $i \in[t]$ and then set $A_{i}=h_{1}^{r_{i}} g_{1}^{a_{i}}, B_{i}=h_{2}^{s_{i}} g_{2}^{b_{i}}$. Finally, set $\mathrm{td}=\left(\left\{a_{i}, r_{i}, s_{i}, b_{i}\right\}_{i=1}^{t}\right)$, pek $=$ $\left\{A_{i}, B_{i}\right\}_{i=1}^{t}$, and output (td, pek).

H.TrapEval(td, $X)$ : let $X=(i, j)$, and then return the coefficients of the degree-2 polynomial

$$
c_{X}\left(y_{1}, y_{2}\right)=\left(y_{1} r_{i}+a_{i}\right)\left(y_{2} s_{j}+b_{j}\right)
$$

First, it is easy to see that the two algorithms satisfy the syntax and correctness properties. Also, in the case $h_{1}=1$ (i.e., $y_{1}=0$ ) or $h_{1}=g_{1}$ (i.e., $y_{1}=1$ ), we obtain a degree-1 polynomial $c_{X}\left(y_{2}\right)$. Second, observe that each element $A_{i}$ (resp. 
$\left.B_{i}\right)$ in pek is a uniformly distributed group element in $\mathbb{G}_{1}$ (resp. $\mathbb{G}_{2}$ ), as in H.Gen, hence $\gamma=0$. Third, we show that the function satisfies the pseudorandomness property under the assumption that XDDH holds in $\mathbb{G}_{1}$. The main observation is that for every $X=(i, j)$, we have $c_{X, 0}=a_{i} b_{j}$ where all the values $b_{i}$ are uniformly distributed and information-theoretically hidden to an adversary who only sees pek. In particular, this holds even if $h_{1}=1$.

To prove the pseudorandomness we make use of Lemma 8 below, which shows that for a uniformly random choice of $\vec{a}, \vec{b} \stackrel{\$}{\leftarrow} \mathbb{Z}_{p}^{t}, c \stackrel{\$}{\leftarrow} \mathbb{Z}_{p}^{t \times t}$ the distributions $\left(g_{1}^{\vec{a}}, g_{1}^{\vec{a} \cdot \vec{b}^{\top}}\right) \in \mathbb{G}_{1}^{t \times(t+1)}$ and $\left(g_{1}^{\vec{a}}, g_{1}^{c}\right) \in \mathbb{G}_{1}^{t \times(t+1)}$ are computationally indistinguishable.

Lemma 8. Let $\vec{a}, \vec{b} \stackrel{\$}{\leftarrow} \mathbb{Z}_{p}^{t}, c \stackrel{\$}{\leftarrow} \mathbb{Z}_{p}^{t \times t}$ be chosen uniformly at random. If the $X D D H$ assumption is $\epsilon^{\prime}$-hard in $\mathbb{G}_{1}$, then for any PPT $\mathcal{B}$ it holds $\mid \operatorname{Pr}\left[\mathcal{B}\left(g_{1}^{\vec{a}}, g_{1}^{\vec{a} \cdot \vec{b}^{\top}}\right)\right.$ $=1]-\operatorname{Pr}\left[\mathcal{B}\left(g_{1}^{\vec{a}}, g_{1}^{c}\right)=1\right] \mid \leq T \cdot \epsilon^{\prime}$.

We first show how to use Lemma 8 to prove that $\mathrm{H}_{\text {sqrt }}$ has programmable pseudorandomness. The proof of Lemma 8 appears slightly below.

Let $\mathcal{A}$ be an adversary that breaks the $\epsilon$-programmable pseudorandomness of $\mathrm{H}_{\text {sqrt }}$. We construct a simulator $\mathcal{B}$ that can distinguish the two distributions $\left(g_{1}^{\vec{a}}, g_{1}^{\vec{a} \cdot \vec{b}^{\top}}\right)$ and $\left(g_{1}^{\vec{a}}, g_{1}^{c}\right)$ described above with advantage greater than $\epsilon$.

$\mathcal{B}$ 's input is a tuple $\left(\overrightarrow{A^{\prime}}, C\right) \in \mathbb{G}_{1}^{t} \times \mathbb{G}_{1}^{t \times t}$ and its goal is to decide about the distribution of $C$. First, $\mathcal{B}$ runs $\mathcal{A}$ (bgp) which outputs the generators $h_{1}, h_{2}$. $\mathcal{B}$ then samples two random vectors $\vec{r}, \vec{\beta} \stackrel{\$}{\leftarrow} \mathbb{Z}_{p}^{t}$, computes $\vec{B}=g_{2}^{\vec{\beta}} \in \mathbb{G}_{2}^{t}, \vec{A}=$ $h_{1}^{\vec{r}} \cdot \overrightarrow{A^{\prime}} \in \mathbb{G}_{1}^{t}$, sets pek $=(\vec{A}, \vec{B})$, and runs $\mathcal{A}$ (pek) Next, for every oracle query $(i, j)$ made by $\mathcal{A}, \mathcal{B}$ simulates the answer by returning to $\mathcal{A}: \mathrm{H}(i, j)=A_{i}^{\beta_{j}}$ and $C_{i, j}$. It is easy to see that if $C=g_{1}^{\vec{a} \cdot \vec{b}^{\top}}$ then $\mathcal{B}$ is perfectly simulating $\operatorname{Exp}_{\mathcal{A}, \mathrm{H}_{\text {sqrt }}}^{P R H-0}$, otherwise, if $C$ is random and independent, then $\mathcal{B}$ is simulating $\operatorname{Exp}_{\mathcal{A}, \mathrm{H}_{\text {sart }}}^{P R H-1}$. As a final note, we observe that the above proof works even in the case $h_{1}=1$.

Proof of Lemma 8. To prove the above lemma, we define $T+1$ hybrid distributions as follows. Let $\vec{a}, \vec{b} \stackrel{\$}{\leftarrow} \mathbb{Z}_{p}^{t}$ and $c \stackrel{\$}{\leftarrow} \mathbb{Z}_{p}^{t \times t}$ be randomly chosen. For every $0 \leq k \leq T$ we define the matrix $\mathcal{M}_{k} \in \mathbb{G}_{1}^{t \times t}$ by specifying the value $\mathcal{M}_{k}[i, j]$ of each entry $(i, j) \in[t] \times[t]$ of the matrix. For every $k^{\prime} \in[T]$, let $(i, j) \in[t] \times[t]$ be such that $k^{\prime}=j+(i-1) t$. Then:

- If $k^{\prime} \leq k, \mathcal{M}_{k}[i, j]=g_{1}^{c_{i, j}}$,

- If $k^{\prime}>k, \mathcal{M}_{k}[i, j]=g_{1}^{a_{i} b_{j}}$.

Notice that $\mathcal{M}_{0}=g_{1}^{\vec{a} \cdot \vec{b}^{\top}}$ while $\mathcal{M}_{T}=g_{1}^{c}$. Moreover,

$$
\begin{gathered}
\left|\operatorname{Pr}\left[\mathcal{B}\left(g_{1}^{\vec{a}}, \mathcal{M}_{0}\right)=1\right]-\operatorname{Pr}\left[\mathcal{B}\left(g_{1}^{\vec{a}}, \mathcal{M}_{T}\right)=1\right]\right| \leq \\
\leq \sum_{k=1}^{T}\left|\operatorname{Pr}\left[\mathcal{B}\left(g_{1}^{\vec{a}}, \mathcal{M}_{k-1}\right)=1\right]-\operatorname{Pr}\left[\mathcal{B}\left(g_{1}^{\vec{a}}, \mathcal{M}_{k}\right)=1\right]\right|
\end{gathered}
$$


Chapter 3. Improving Efficiency: Linearly Homomorphic Signatures with Sublinear Public Keys via APHFs

$$
\leq T \cdot\left|\operatorname{Pr}\left[\mathcal{B}\left(g_{1}^{\vec{a}}, \mathcal{M}_{k-1}\right)=1\right]-\operatorname{Pr}\left[\mathcal{B}\left(g_{1}^{\vec{a}}, \mathcal{M}_{k}\right)=1\right]\right| .
$$

We complete the proof of Lemma 8 by showing the following claim:

Claim 1. For every $1 \leq k \leq T$, if $X D D H$ is $\epsilon^{\prime}$-hard in $\mathbb{G}_{1}$, then

$$
\mid \operatorname{Pr}\left[\mathcal{B}\left(g_{1}^{\vec{a}}, \mathcal{M}_{k-1}=1\right]-\operatorname{Pr}\left[\mathcal{B}\left(g_{1}^{\vec{a}}, \mathcal{M}_{k}\right)=1\right] \mid \leq \epsilon^{\prime}\right.
$$

Assume by contradiction that $\mid \operatorname{Pr}\left[\mathcal{B}\left(g_{1}^{\vec{a}}, \mathcal{M}_{k-1}=1\right]-\operatorname{Pr}\left[\mathcal{B}\left(g_{1}^{\vec{a}}, \mathcal{M}_{k}\right)=1\right] \mid \geq \epsilon^{\prime}\right.$. Then it is possible to build a simulator $\mathcal{B}^{\prime}$ which breaks the XDDH assumption in $\mathbb{G}_{1}$ with advantage greater than $\epsilon^{\prime} . \mathcal{B}^{\prime}$ gets an XDDH instance $\left(g_{1}, g_{1}^{\alpha}, g_{1}^{\beta}, g_{1}^{\gamma}\right)$ and proceeds as follows:

- It samples $c_{1}, \ldots, c_{k-1} \stackrel{\$}{\leftarrow} \mathbb{Z}_{p}$.

- It samples $a_{1}, \ldots, a_{\hat{i}-1}, a_{\hat{i}+1}, \ldots, a_{t}, b_{1}, \ldots, b_{\hat{j}-1}, b_{\hat{j}+1}, \ldots, b_{t} \stackrel{\$}{\leftarrow} \mathbb{Z}_{p}$, where $(\hat{i}, \hat{j})$ correspond to $k$, i.e., $k=\hat{j}+(\hat{i}-1) t$.

- It implicitly sets $\tilde{\vec{a}}=\left(a_{1}, \ldots, a_{\hat{i}-1}, \alpha, a_{\hat{i}+1}, \ldots a_{t}\right)$ and $\tilde{\vec{b}}=\left(b_{1}, \ldots, b_{\hat{j}-1}, \beta\right.$, $\left.b_{\hat{j}+1}, \ldots b_{t}\right)$.

- $\mathcal{B}^{\prime}$ builds a matrix $\mathcal{M} \in \mathbb{G}_{1}^{t \times t}$ where:

- If $k^{\prime} \leq k-1, \mathcal{M}[i, j]=g_{1}^{c_{i, j}}$,

- If $k^{\prime}=k, \mathcal{M}[i, j]=g_{1}^{\gamma}$,

- If $k^{\prime}>k, \mathcal{M}[i, j]=g_{1}^{\tilde{a}_{i} \cdot \tilde{b}_{j}}$. Notice that such value can be efficiently computed by $\mathcal{B}^{\prime}$ as it knows $g_{1}^{\tilde{a}_{\hat{i}}}=g_{1}^{\alpha}, g_{1}^{\tilde{b}_{\hat{j}}}=g_{1}^{\beta}, \tilde{a}_{i}, \forall i \neq \hat{i}, \tilde{b}_{j}, \forall j \neq \hat{j}$, and $k^{\prime}>k$ implies $(i, j) \neq(\hat{i}, \hat{j})$.

- $\mathcal{B}^{\prime}$ runs $b^{\prime} \leftarrow \mathcal{B}\left(g_{1}^{\vec{a}}, \mathcal{M}\right)$ and returns the same bit $b^{\prime}$.

As one can check, if $\gamma=\alpha \beta$, then $\mathcal{M}^{\prime}$ is distributed as $\mathcal{M}_{k-1}$. Otherwise, if $\gamma$ is random and independent, $\mathcal{M}^{\prime}$ is distributed as $\mathcal{M}_{k}$. Therefore,

$$
\begin{aligned}
& \left|\operatorname{Pr}\left[\mathcal{B}^{\prime}\left(g_{1}, g_{1}^{\alpha}, g_{1}^{\beta}, g_{1}^{\alpha \beta}\right)=1\right]-\operatorname{Pr}\left[\mathcal{B}^{\prime}\left(g_{1}, g_{1}^{\alpha}, g_{1}^{\beta}, g_{1}^{\gamma}\right)=1\right]\right|= \\
& \quad=\left|\operatorname{Pr}\left[\mathcal{B}\left(g_{1}^{\tilde{\tilde{a}}}, \mathcal{M}_{k-1}\right)=1\right]-\operatorname{Pr}\left[\mathcal{B}\left(g_{1}^{\tilde{\tilde{a}}}, \tilde{\mathcal{M}}_{k}\right)=1\right]\right| \geq \epsilon^{\prime}
\end{aligned}
$$

(poly, 0, 2)-programmability of $\boldsymbol{H}_{\text {sqrt }}$. Below we show that $\boldsymbol{H}_{\text {sqrt }}$ is (poly, 0,2 , $\gamma, \delta$ )-programmable for $\gamma=0$ and $\delta=1$. While such (poly, 0 )-programmability might look uninteresting at first, this property turns out to be useful in various security proofs, as shown in our application to homomorphic signatures of Section 3.4 , 
Chapter 3. Improving Efficiency: Linearly Homomorphic Signatures with Sublinear Public Keys via APHFs

Theorem 8. The asymmetric hash function $\mathrm{H}_{\text {sqrt }}$ described above is (poly, $0, d, \gamma$, $\delta)$-programmable with $d=2, \gamma=0$ and $\delta=1$. Furthermore, in the case when either $\hat{h}_{1}=\hat{g}_{1}$ or $\hat{h}_{2}=\hat{g}_{2}, \mathrm{H}_{\text {sqrt }}$ is (poly, $0, d, \gamma, \delta$ )-programmable with $d=1, \gamma=0$ and $\delta=1$.

Proof. The trapdoor algorithms are defined as follows:

H.TrapGen $\left(1^{\lambda}, \hat{g}_{1}, \hat{h}_{1}, \hat{g}_{2}, \hat{h}_{2}\right): \quad$ first, sample $r_{i}, s_{i} \stackrel{\$}{\leftarrow} \mathbb{Z}_{p}$ for all $i \in[t]$ and then set $A_{i}=\hat{h}_{1}^{r_{i}}, B_{i}=\hat{h}_{2}^{s_{i}}$. Finally, set $\mathrm{td}=\left(\left\{r_{i}, s_{i}\right\}_{i=1}^{t}\right)$, pek $=\left\{A_{i}, B_{i}\right\}_{i=1}^{t}$, and output (td, pek).

H.TrapEval(td, $X)$ : let $X=(i, j)$, and then return the coefficients of the degree-2 polynomial

$$
c_{X}\left(y_{1}, y_{2}\right)=\left(y_{1} y_{2}\right) r_{i} s_{j}
$$

Syntax and correctness are easily seen by inspection. The public key generated by H.TrapGen is distributed identically to the one generated by H.Gen, from which $\gamma=0$. Also, it is clear that for any $X \in \mathcal{X}$, the degree-0 term of the polynomial $c_{X}$ computed by H.TrapEval is always 0 . It is straightforward to see that in the case $y_{1}=1$ ( or $y_{2}=1$ ) the function satisfies the programmability with $d=1$.

Weak (poly, 1,2)-programmability of $\mathrm{H}_{\text {sqrt }}$. Here we prove that $\mathrm{H}_{\text {sqrt }}$ is weakly (poly, $1,2, \gamma, \delta$ )-programmable for $\gamma=0$ and $\delta=1$.

Theorem 9. The asymmetric hash function $\mathrm{H}_{\text {sqrt }}$ described above is weakly (poly, $1, d, \gamma, \delta)$-programmable with $d=2, \gamma=0$ and $\delta=1$.

Proof. The trapdoor algorithms are defined as follows:

H.TrapGen $\left(1^{\lambda}, \hat{g}_{1}, \hat{h}_{1}, \hat{g}_{2}, \hat{h}_{2}, Z\right)$ : let $Z=\left(i^{*}, j^{*}\right) \in[t] \times[t]$. First, sample $r_{i}, s_{i} \stackrel{\&}{\leftarrow}$ $\mathbb{Z}_{p}$ for all $i \in[t]$. Next, compute $A_{i^{*}}=\hat{g}_{1} \hat{h}_{1}^{r_{i^{*}}}, B_{j^{*}}=\hat{g}_{2} \hat{h}_{2}^{s_{j^{*}}}, A_{i}=\hat{h}_{1}^{r_{i}}, \forall i \neq i^{*}$ and $B_{j}=\hat{h}_{2}^{s_{j}}, \forall j \neq j^{*}$. Finally, set $\mathrm{td}=\left(\left\{r_{i}, s_{i}\right\}_{i=1}^{t}\right)$, pek $=\left\{A_{i}, B_{i}\right\}_{i=1}^{t}$, and output (td, pek).

H.TrapEval(td, $X)$ : given $X=(i, j)$, return the coefficients of the degree-2 polynomial

$$
c_{X}\left(y_{1}, y_{2}\right)=\alpha_{i}\left(y_{1}\right) \cdot \beta_{j}\left(y_{2}\right)
$$

where $\alpha_{i}\left(y_{1}\right)$ (resp. $\left.\beta_{j}\left(y_{2}\right)\right)$ is the discrete logarithm of $A_{i}\left(\right.$ resp. $\left.B_{j}\right)$ in base $\hat{g}_{1}$ (resp. $\hat{g}_{2}$ ), viewed as a degree-1 polynomial in the unknown $y_{1}$ (resp. $y_{2}$ ).

Syntax and correctness are easily seen by inspection. The public key generated by H.TrapGen is distributed identically to the one generated by H.Gen, from which $\gamma=0$. Also, it is clear from the construction that for $Z=\left(i^{*}, j^{*}\right)$ we have $c_{Z}\left(y_{1}, y_{2}\right)=\left(y_{1} r_{1}+1\right)\left(y_{2} s_{j}+1\right)$, and thus $c_{Z, 0}=1$, whereas for every $X \neq Z$ the degree- 0 term of the polynomial $c_{Z}\left(y_{1}, y_{2}\right)$ computed by $\mathrm{H}$. TrapEval is always 0 . And this holds with probability $\delta=1$. 
Chapter 3. Improving Efficiency: Linearly Homomorphic Signatures with Sublinear Public Keys via APHFs

Weak (poly, 1, 2)-degree-2-programmability of $\mathrm{H}_{\text {sqrt }}$. Finally, we prove that $\mathrm{H}_{\text {sqrt }}$ is also weakly (poly, $1,2, \gamma, \delta$ )-degree-2-programmable for $\gamma=0$ and $\delta=1$.

Theorem 10. The asymmetric hash function $\mathrm{H}_{\text {sqrt }}$ described above is weakly (poly, $1, d, \gamma, \delta$ )-degree-2 programmable with $d=2, \gamma=0$ and $\delta=1$.

Proof. The proof of this theorem can be seen as the "dual" version of the one of Theorem 9. Instead of setting the simulated keys so that $Z$ is the only input for which $c_{Z, 0}=1$, here the keys are simulated in such a way that $Z$ is the only input in which the term $y_{1} y_{2}$ appears. More precisely, the trapdoor algorithms work as follows:

H.TrapGen $\left(1^{\lambda}, \hat{g}_{1}, \hat{h}_{1}, \hat{g}_{2}, \hat{h}_{2}, Z\right)$ : let $Z=\left(i^{*}, j^{*}\right) \in[t] \times[t]$. First, sample $r_{i}, s_{i} \stackrel{\$}{\leftarrow}$ $\mathbb{Z}_{p}$ for all $i \in[t]$ and then set $A_{i^{*}}=\hat{h}_{1} \hat{g}_{1}^{r_{i^{*}}}, B_{j^{*}}=\hat{h}_{2} \hat{g}_{2}^{s^{*}}, A_{i}=\hat{g}_{1}^{r_{i}}, \forall i \neq i^{*}$ and $B_{j}=\hat{g}_{2}^{s_{j}}, \forall j \neq j^{*}$. Finally, set $\mathrm{td}=\left(\left\{r_{i}, s_{i}\right\}_{i=1}^{t}\right)$, pek $=\left\{A_{i}, B_{i}\right\}_{i=1}^{t}$, and output (td, pek).

H.TrapEval(td, $X)$ : let $X=(i, j)$, and then return the coefficients of the degree-2 polynomial

$$
c_{X}\left(y_{1}, y_{2}\right)=\alpha_{i}\left(y_{1}\right) \cdot \beta_{j}\left(y_{2}\right)
$$

where $\alpha_{i}\left(y_{1}\right)$ (resp. $\left.\beta_{j}\left(y_{2}\right)\right)$ is the discrete logarithm of $A_{i}$ (resp. $\left.B_{j}\right)$ in base $\hat{g}_{1}$ (resp. $\left.\hat{g}_{2}\right)$, viewed as a degree- 1 polynomial in the unknown $y_{1}$ (resp. $y_{2}$ ).

Syntax and correctness are easily seen by inspection. The public key generated by $\mathrm{H}$.TrapGen is distributed identically to the one generated by H.Gen, from which $\gamma=0$. By construction, we have that for $Z=\left(i^{*}, j^{*}\right), c_{Z}\left(y_{1}, y_{2}\right)=\left(r_{1}+y_{1}\right)\left(s_{j}+\right.$ $\left.y_{2}\right)$, and thus $c_{Z, 2}=1$, whereas for every $X \neq Z$ the polynomial $c_{X}\left(y_{1}, y_{2}\right)$ has degree $\leq 1$, and thus $c_{X, 2}=0$. This property holds with probability $\delta=$ 1.

\subsection{Linearly-Homomorphic Signatures with Short Public Keys}

In this section, we show a new linearly-homomorphic signature scheme that uses APHFs in a generic way. By instantiating the APHFs with our construction $\mathrm{H}_{\text {sqrt }}$ given in Section 3.3, we obtain the first linearly-homomorphic signature scheme that is secure in the standard model, and whose public key has a size that is sublinear in both the dataset size and the dimension of the signed vectors. Precisely, if the signature scheme supports datasets of maximal size $N$ and can sign vectors of dimension $T$, then the public key of our scheme is of size $O(\sqrt{N}+\sqrt{T})$. All previously existing constructions in the standard model achieved only public keys of length $O(N+T)$. Furthermore, our scheme is adaptive secure and achieves the interesting property of efficient verification that allows to use the scheme for verifiable delegation of computation in the preprocessing model [49]. 
Chapter 3. Improving Efficiency: Linearly Homomorphic Signatures with Sublinear Public Keys via APHFs

\subsubsection{Our Construction}

Let $\Sigma^{\prime}=\left(\right.$ KeyGen $^{\prime}$, Sign' $\left.^{\prime}, V_{e r}^{\prime}\right)$ be a regular signature scheme, and $F: \mathcal{K} \times$ $\{0,1\}^{*} \rightarrow \mathbb{Z}_{p}$ be a pseudorandom function with key space $\mathcal{K}$. Our linearlyhomomorphic signature scheme signs $T$-dimensional vectors of messages in $\mathbb{Z}_{p}$, supports datasets of size $N$, with both $N=\operatorname{poly}(\lambda)$ and $T=\operatorname{poly}(\lambda)$ and is context-hiding secure. Let $\mathrm{H}=\left(\mathrm{H} . \mathrm{Gen}, \mathrm{H}\right.$.PriEval, H.PubEval) and $\mathrm{H}^{\prime}=\left(\mathrm{H} . G e n^{\prime}\right.$, H.PriEval', H.PubEval') be two asymmetric programmable hash functions such that $\mathrm{H}:[N] \rightarrow \mathbb{G}_{1}$ and $\mathrm{H}^{\prime}:[T] \rightarrow \mathbb{G}_{1}$.

We construct a homomorphic signature HSig $=($ KeyGen, Sign, Ver, Eval) as follows:

KeyGen $\left(1^{\lambda}, \mathcal{L}, T\right)$. Let $\lambda$ be the security parameter, $\mathcal{L}$ be a set of admissible labels where $\mathcal{L}=\{1, \ldots, N\}$, and $T$ be an integer representing the dimension of the vectors to be signed. The key generation algorithm works as follows.

- Generate a key pair $\left(\mathrm{vk}^{\prime}, \mathrm{sk}^{\prime}\right) \stackrel{\$}{\leftarrow} \operatorname{KeyGen}\left(1^{\lambda}\right)$ for the regular scheme.

- Run bgp $\stackrel{\$}{\leftarrow} \mathcal{G}\left(1^{\lambda}\right)$ to generate the bilinear groups parameters bgp = $\left(p, \mathbb{G}_{1}, \mathbb{G}_{2}, \mathbb{G}_{T}, g_{1}, g_{2}, e\right)$ where $\mathbb{G}_{1}, \mathbb{G}_{2}$ and $\mathbb{G}_{T}$ are groups of prime order $p \approx 2^{\lambda}, g_{1} \in \mathbb{G}_{1}, g_{2} \in \mathbb{G}_{2}$ are generators and $e: \mathbb{G}_{1} \times \mathbb{G}_{2} \rightarrow \mathbb{G}_{T}$ is an efficiently computable, non-degenerate bilinear map.

- Choose two random seeds $K, \hat{K} \stackrel{\$}{\leftarrow} \mathcal{K}$ for the PRF $F: \mathcal{K} \times\{0,1\}^{*} \rightarrow \mathbb{Z}_{p}$.

- Run (sek, pek) $\stackrel{\$}{\leftarrow}$ H.Gen $\left(1^{\lambda}\right.$, bgp $)$ and $\left(\right.$ sek $^{\prime}$, pek $\left.^{\prime}\right) \stackrel{\$}{\leftarrow}$ H.Gen $\left(1^{\lambda}\right.$, bgp $)$ to generate the keys of the asymmetric hash functions.

- Return $\mathrm{vk}=\left(\mathrm{vk}^{\prime}\right.$, bgp, pek, pek' $)$ and $\mathrm{sk}=\left(\mathrm{sk}^{\prime}, K, \hat{K}\right.$, sek, sek' $)$.

$\operatorname{Sign}(\mathrm{sk}, \Delta, \tau, \vec{m})$. The signing algorithm takes as input the secret key sk, a data set identifier $\Delta \in\{0,1\}^{*}$, a label $\tau \in[N]$ and a message vector $\vec{m} \in \mathbb{Z}_{p}^{T}$, and proceeds as follows:

1. Derive the integer $z \leftarrow F_{K}(\Delta)$ using the PRF, and compute $Z=g_{2}^{z}$.

2. Compute $\sigma_{\Delta} \leftarrow \operatorname{Sign}^{\prime}\left(\mathbf{s k}^{\prime}, \Delta \mid Z\right)$ to bind $Z$ to the dataset identifier $\Delta$.

3. Derive $r \leftarrow F_{\hat{K}}(\Delta \mid \tau)$, set $R=g_{1}^{r}$ and compute

$$
S=\left(\text { H.PriEval }(\text { sek, } \tau) \cdot R \cdot \prod_{j=1}^{T} \text { H.PriEval' }\left(\text { sek }^{\prime}, j\right)^{m_{j}}\right)^{1 / z}
$$

4. Return a signature $\sigma=\left(\sigma_{\Delta}, Z, R, S\right)$.

Essentially, the algorithm consists of two main steps. First, it uses the PRF $F_{K}$ to derive a common parameter $z$ which is related to the data set $\Delta$, and it signs the public part, $Z=g_{2}^{z}$, of this parameter using the 
Chapter 3. Improving Efficiency: Linearly Homomorphic Signatures with Sublinear Public Keys via APHFs

signature scheme $\Sigma^{\prime}$. Then it uses the same PRF $F_{\hat{K}}$ with a different seed $\hat{K}$ to create the pseudorandom element $R=g_{1}^{F_{\hat{K}}(\Delta \mid \tau)}$. Second, it uses $z$ to create the homomorphic component $S$ of the signature, such that $S$ is now related to all $(\Delta, \tau, \vec{m})$.

Eval(vk, $f, \vec{\sigma})$. The public evaluation algorithm takes as input the public key $\mathrm{vk}$, a linear function $f: \mathbb{Z}_{p}^{\ell} \rightarrow \mathbb{Z}_{p}$ described by its vector of coefficients $\vec{f}=\left(f_{1}, \ldots, f_{\ell}\right)$, and a vector $\vec{\sigma}$ of $\ell$ signatures $\sigma_{1}, \ldots, \sigma_{\ell}$ where $\sigma_{i}=$ $\left(\sigma_{\Delta, i}, Z_{i}, R_{i}, S_{i}\right)$ for $i=1, \ldots, \ell$. Eval returns a signature $\sigma=\left(\sigma_{\Delta}, Z, R, S\right)$ that is obtained by setting $Z=Z_{1}, \sigma_{\Delta}=\sigma_{\Delta, 1}$, and by computing

$$
R=\prod_{i=1}^{\ell} R_{i}^{f_{i}}, \quad S=\prod_{i=1}^{\ell} S_{i}^{f_{i}}
$$

$\operatorname{Ver}\left(\mathrm{vk}, \mathcal{P}_{\Delta}, \vec{m}, \sigma\right)$. Let $\mathcal{P}_{\Delta}=\left(\left(f, \tau_{1}, \ldots, \tau_{\ell}\right), \Delta\right)$ be a multi-labeled program such that $f: \mathbb{Z}_{p}^{\ell} \rightarrow \mathbb{Z}_{p}$ is a linear function described by coefficients $\vec{f}=\left(f_{1}, \ldots, f_{\ell}\right)$. Let $\vec{m} \in \mathbb{Z}_{p}^{T}$ be a message-vector and $\sigma=\left(\sigma_{\Delta}, Z, R, S\right)$ be a signature.

First, run $\operatorname{Ver}^{\prime}\left(\mathrm{vk}^{\prime}, \Delta \mid Z, \sigma_{\Delta}\right)$ to check that $\sigma_{\Delta}$ is a valid signature for $Z$ and the dataset identifier $\Delta$ taken as input by the verification algorithm. If $\sigma_{\Delta}$ is not valid, stop and return 0 (reject).

Otherwise, output 1 if and only if the following equation is satisfied

$$
e(S, Z)=\left(\prod_{i=1}^{\ell} \text { H.PubEval }\left(\text { pek, } \tau_{i}\right)^{f_{i}}\right) \cdot e\left(R, g_{2}\right) \cdot\left(\prod_{j=1}^{T} \text { H.PubEval }\left(\text { pek }^{\prime}, j\right)^{m_{j}}\right)
$$

Finally, we describe the algorithms for efficient verification:

VerPrep $(\mathbf{v k}, \mathcal{P})$. Let $\mathcal{P}=\left(f, \tau_{1}, \ldots, \tau_{\ell}\right)$ be a labeled program for a linear function $f: \mathbb{Z}_{p}^{\ell} \rightarrow \mathbb{Z}_{p}$. The algorithm computes $H=\prod_{i=1}^{\ell}$ H.PubEval $\left(\text { pek, } \tau_{i}\right)^{f_{i}}$, and returns the concise verification key $\mathrm{vk}_{\mathcal{P}}=\left(\mathrm{vk}^{\prime}, \mathrm{bgp}, H \text {, pek' }\right)^{\prime}$.

$\operatorname{EffVer}\left(\operatorname{vk}_{\mathcal{P}}, \Delta, \vec{m}, \sigma\right)$. The online verification is the same as Ver except that in the verification equation the value $H$ has been already computed in the off-line phase (and is included in $\mathbf{v k}_{\mathcal{P}}$ ).

Clearly, running the combination of VerPrep and EffVer gives the same result as running Ver, and EffVer's running time is independent of $f$ 's complexity $\ell$.

We formally show the correctness of our homomorphic signature scheme in Section 3.4.2. The following theorems state the security and context-hiding of our scheme. Their proofs appear in Section 3.4.3 and Section 3.4.4 respectively. 
Chapter 3. Improving Efficiency: Linearly Homomorphic Signatures with Sublinear Public Keys via APHFs

Theorem 11 (Unforgeability of HSig). Assume that $\Sigma^{\prime}$ is an unforgeable signature scheme, $F$ is a pseudorandom function, and $\mathcal{G}$ is a bilinear group generator such that: $\mathbf{H}$ has $(1, \gamma, \epsilon)$-programmable pseudorandomness; $\mathbf{H}^{\prime}$ is weakly (poly, $1,2, \gamma^{\prime}, \delta^{\prime}$ )-programmable and (poly, $0,1, \gamma^{\prime}, \delta^{\prime}$ )-programmable; the 2 -DHI and the FDHI assumptions hold. Then $\mathrm{HSig}$ is a secure linearly-homomorphic signature scheme.

We note that our scheme HSig can be instantiated by instantiating both $\mathrm{H}$ and $\mathrm{H}^{\prime}$ with two different instances of our programmable hash $\mathrm{H}_{\text {sqrt }}$ described in Section 3.3.3. As one can check in Section 3.3.3. $\mathrm{H}_{\text {sqrt }}$ allows for the multiple programmability modes required in our Theorem 11. Let us stress that requiring the same function to have multiple programmability modes is not contradictory, as such modes do not have to hold simultaneously. It simply means that for the same function there exist different pairs of trapdoor algorithms each satisfying programmability with different parameters. $5^{5}$

Theorem 12 (Context-Hiding of HSig). Assume that $\Sigma^{\prime}$ is an unforgeable signa-

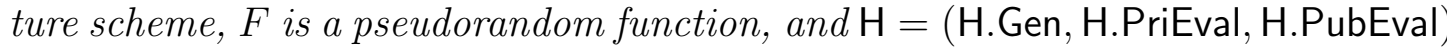
and $\mathrm{H}^{\prime}=\left(\mathrm{H} . \mathrm{Gen}^{\prime}, \mathrm{H}\right.$. PriEval', H.PubEval') be two asymmetric programmable hash functions such that $\mathrm{H}:[N] \rightarrow \mathbb{G}_{1}$ and $\mathrm{H}^{\prime}:[T] \rightarrow \mathbb{G}_{1}$. If $\Sigma^{\prime}$ is deterministic, then the scheme HSig satisfies perfect context hiding in the sense of Definition 2.

We note that for the sake of context-hiding in our scheme there are no specific hiding algorithms (i.e., one can see Hide as the identity function and HVerify as Ver). In other words the signing and the evaluation algorithms already produce signatures with context-hiding.

Remark 3 (Alternative requirement for context-hiding). To prove context-hiding of our linearly-homomorphic signature scheme, in Theorem 12 we require the scheme $\Sigma^{\prime}$ to be deterministic. It is worth mentioning that alternatively we could also prove context-hiding by requiring $\Sigma^{\prime}$ to be re-randomizable and by giving an explicit Hide algorithm. In a few words, ${ }^{6}$ a signature scheme is re-randomizable if it comes with an additional algorithm $\mathcal{R}$ that on input a message $m$ and a signature $\sigma$ outputs a new signature $\tilde{\sigma}$ which is indistinguishable from a fresh signature on $m$. If $\Sigma^{\prime}$ is re-randomizable, then context-hiding can be achieved by letting Hide work as follows: instead of simply being the identity function, Hide takes a signature $\sigma=\left(\sigma_{\Delta}, Z, R, S\right)$, applies the re-randomization algorithm on $\sigma_{\Delta}$ to obtain another signature $\tilde{\sigma}_{\Delta}$ on the message $(\Delta \mid Z)$, and outputs $\tilde{\sigma}=$ $\left(\tilde{\sigma}_{\Delta}, Z, R, S\right)$. This way the fresh signature $\sigma_{\Delta}$ created by the simulator will be indistinguishable from the one $\tilde{\sigma}_{\Delta}$ in the output of Hide.

\footnotetext{
${ }^{5}$ We also stress that, by definition, the outputs of these trapdoor algorithms are statistically indistinguishable.

${ }^{6} \mathrm{~A}$ formal definition of re-randomizable signatures can be found in [2].
} 
Chapter 3. Improving Efficiency: Linearly Homomorphic Signatures with Sublinear Public Keys via APHFs

\subsubsection{Proof of Correctness}

Theorem 13. If $\Sigma^{\prime}$ is a correct signature scheme, and $\mathrm{H}, \mathrm{H}^{\prime}$ are asymmetric hash functions for bilinear groups, then the scheme HSig satisfies the authentication correctness property.

Proof. Let (sk, vk) be a pair of honestly generated keys and let $\sigma \leftarrow \operatorname{Sign}($ sk, $\Delta$, $\tau, \vec{m})$ be a honestly generated signature, with $\sigma=\left(\sigma_{\Delta}, Z, R_{\tau}, S_{\tau}\right)$. In order to prove that the verification algorithm $\operatorname{Ver}\left(\mathrm{vk}, \mathcal{I}_{(\Delta, \tau)}, \vec{m}, \sigma\right)$ outputs 1 with all but negligible probability, the first observation to do is that by the correctness of $\Sigma^{\prime}$ the signature $\sigma_{\Delta}$ verifies correctly for $Z$ and $\Delta$. Then, by construction of HSig, we can see that

$$
S_{\tau}=\left(\text { H.PriEval }(\text { sek, } \tau) \cdot R_{\tau} \cdot \prod_{j=1}^{T} \text { H.PriEval' }\left(\text { sek }^{\prime}, j\right)^{m_{j}}\right)^{1 / z}
$$

Hence, we have that

$$
\begin{aligned}
& e\left(S_{\tau}, Z\right)=e\left(\left(\text { H.PriEval }(\text { sek }, \tau) \cdot R_{\tau} \cdot \prod_{j=1}^{T} \text { H.PriEval' }\left(\text { sek }^{\prime}, j\right)^{m_{j}}\right)^{1 / z}, Z\right) \\
& =e\left(\operatorname{H.PriEval}(\mathrm{sek}, \tau) \cdot R_{\tau} \cdot \prod_{j=1}^{T} \operatorname{H.PriEval}\left(\operatorname{sek}^{\prime}, j\right)^{m_{j}}, g_{2}\right) \\
& =e\left(\mathrm{H} . \text { PriEval }(\mathrm{sek}, \tau), g_{2}\right) \cdot e\left(R_{\tau}, g_{2}\right) \cdot e\left(\prod_{j=1}^{T} \mathrm{H} \text {.PriEval' }\left(\text { sek }^{\prime}, j\right)^{m_{j}}, g_{2}\right) \\
& =\text { H.PubEval }(\text { pek, } \tau) \cdot e\left(R_{\tau}, g_{2}\right) \cdot \prod_{j=1}^{T} \text { H.PubEval }{ }^{\prime}\left(\text { pek }^{\prime}, j\right)^{m_{j}}
\end{aligned}
$$

where the last equation holds by definition of H.PubEval and H.PubEval'.

Theorem 14. If $\Sigma^{\prime}$ is a correct signature scheme, and $\mathrm{H}, \mathrm{H}^{\prime}$ are asymmetric hash functions for bilinear groups, then the scheme HSig satisfies the evaluation correctness property.

Proof. Let (sk, vk) be a pair of honestly generated keys, and let $\left\{\vec{m}^{(i)}, \mathcal{P}_{i, \Delta}, \sigma_{i}=\right.$ $\left.\left(\sigma_{\Delta}, Z, R_{i}, S_{i}\right)\right\}_{i=1}^{\ell}$ be messages, labeled programs and signatures such that $\operatorname{Ver}(\mathbf{v k}$, $\left.\mathcal{P}_{i, \Delta}, \vec{m}^{(i)}, \sigma_{i}\right)=1$, for all $i=1$ to $\ell$. Let $\sigma \leftarrow \operatorname{Eval}\left(\mathrm{vk}, f, \vec{\sigma}=\left(\sigma_{1}, \ldots, \sigma_{\ell}\right)\right)$ be a a signature obtained by running Eval on signatures $\left(\sigma_{1}, \ldots, \sigma_{\ell}\right)$, where $\sigma=$ $\left(\sigma_{\Delta}, Z, R, S\right)$. By construction of Eval, we have $R=\prod_{i=1}^{\ell} R_{i}^{f_{i}}$ and $S=\prod_{i=1}^{\ell} S_{i}^{f_{i}}$. So, if we let $\vec{m}=f\left(\vec{m}^{(1)}, \ldots, \vec{m}^{(\ell)}\right)=\sum_{i=1}^{\ell} f_{i} \cdot \vec{m}^{(i)}$, for evaluation correctness we want to prove that the verification algorithm $\operatorname{Ver}\left(\mathrm{vk}, \mathcal{P}_{\Delta}, \vec{m}, \sigma\right)$ outputs 1 . 
Chapter 3. Improving Efficiency: Linearly Homomorphic Signatures with Sublinear Public Keys via APHFs

The fact that $\sigma_{\Delta}$ verifies correctly for $Z$ and $\Delta$ is immediate by correctness of $\Sigma^{\prime}$ and by construction of Eval (which simply copies one of these honestlygenerated signatures).

Since each $\sigma_{i}$ verifies correctly, for every $i=1, \ldots, \ell$ we have

$$
e\left(S_{i}, Z\right)=\text { H.PubEval }\left(\text { pek, } \tau_{i}\right) \cdot e\left(R_{i}, g_{2}\right) \cdot \prod_{j=1}^{T} \text { H.PubEval' }\left(\text { pek }^{\prime}, j\right)^{m_{j}^{(i)}}
$$

Then, by the previous equations and the fact that $\mathrm{H}, \mathrm{H}^{\prime}$ are asymmetric hash functions for bilinear groups, we obtain the desired equation:

$$
\begin{aligned}
e(S, Z) & =e\left(\prod_{i=1}^{\ell} S_{i}^{f_{i}}, Z\right) \\
& =e\left(\prod_{i=1}^{\ell}\left(\text { H.PriEval }(\text { sek, } \tau)^{f_{i}} \cdot R_{i}^{f_{i}} \cdot \prod_{j=1}^{T} \mathrm{H}_{\text {PriEval }}\left(\text { sek }^{\prime}, j\right)^{f_{i} m_{j}^{(i)}}\right)^{1 / z}, Z\right) \\
& =\left(\prod_{i=1}^{\ell} \mathrm{H} . \text { PubEval }(\text { pek, } \tau)^{f_{i}}\right) \cdot e\left(R, g_{2}\right) \cdot\left(\prod_{j=1}^{T} \mathrm{H} . P u b E v a l^{\prime}\left(\text { pek }^{\prime}, j\right)^{\sum_{i=1}^{\ell} f_{i} m_{j}^{(i)}}\right) \\
& =\left(\prod_{i=1}^{\ell} \mathrm{H} . \text { PubEval }(\text { pek, } \tau)^{f_{i}}\right) \cdot e\left(R, g_{2}\right) \cdot\left(\prod_{j=1}^{T} \mathrm{H} . P u b E v a l^{\prime}\left(\text { pek }^{\prime}, j\right)^{m_{j}}\right)
\end{aligned}
$$

\subsubsection{Proof of Security}

To prove Theorem 11, we show that for every PPT adversary $\mathcal{A}$ running in the security experiment HomUF-CMA ${ }_{\mathcal{A}, \mathrm{HSig}}$, the probability that the experiment outputs 1 is negligible. We do the proof by describing a series of hybrid games. We write $G_{i}(\mathcal{A})$ to denote the event that a run of Game $i$ with adversary $\mathcal{A}$ returns 1 . Some of the games use some flag values $\mathcal{A}_{i}$ that are initially set to false. If at the end of a game any of these values is set to true, the game simply outputs 0. We call $\operatorname{Bad}_{i}$ the event that $\mathcal{A}_{i}$ is set to true during the run of an experiment. Essentially, whenever an event $\operatorname{Bad}_{i}$ occurs in Game $i$, the game may deviate its outcome.

Before going into the proof, we recall that, as proved by Freeman in [63, in a linearly-homomorphic signature scheme any adversary who outputs a Type 3 forgery can be converted into one that outputs a Type 2 one.

Proposition 1 ([63]). Let HSig be a linearly homomorphic signature scheme with message space $\mathcal{M} \subset \mathcal{R}^{n}$ for some ring $\mathcal{R}$. If $\mathrm{HSig}$ is secure against Type 2 forgeries, then HSig is secure against Type 3 forgeries. 
Chapter 3. Improving Efficiency: Linearly Homomorphic Signatures with Sublinear Public Keys via APHFs

That is the reason why in the following proof we only deal with Type- 1 and Type-2 forgeries.

Game 0 This game is the security experiment HomUF-CMA $\mathcal{A}_{\mathcal{A}, \mathrm{HSig}}$ (where $\mathcal{A}$ only outputs Type-1 or Type-2 forgeries).

Game 1 This game is defined as Game 0 apart from the fact that whenever $\mathcal{A}$ returns a forgery $\sigma^{*}=\left(\sigma_{\Delta}^{*}, Z^{*}, R^{*}, S^{*}\right)$ such that $Z^{*}$ was not generated by the challenger in the signing query phase, then Game 1 sets $\mathcal{A}_{1} \leftarrow$ true. As we show in Lemma 9, any noticeable difference between Game 0 and Game 1 can be reduced to producing a forgery for the regular signature scheme $\Sigma^{\prime}$. Furthermore, it is worth noting that after this change, the game never outputs 1 if the adversary returns a Type- 1 forgery.

Game 2 This game is defined as Game 1 except that the pseudorandom function $F$ is replaced by a random function $\mathcal{R}:\{0,1\}^{*} \rightarrow \mathbb{Z}_{p}$. It is easy to see that Game 1 is computationally indistinguishable from Game 2 under the assumption that $F$ is pseudorandom.

Game 3 is defined as Game 2 except for the following change. Let $\left(\mathcal{P}_{\Delta^{*}}^{*}, \sigma^{*}, \vec{m}^{*}\right)$ be the forgery returned by the adversary where $\mathcal{P}_{\Delta^{*}}^{*}=\left(\overrightarrow{f^{*}}, \mathcal{L}^{*}\right), \sigma^{*}=$ $\left(\sigma_{\Delta}^{*}, Z^{*}, R^{*}, S^{*}\right)$ and $\Delta^{*}=\Delta_{\mu}$ for some $\mu \in[Q]$ where $Q$ is the number of distinct datasets asked by $\mathcal{A}$ during the game (note that such $\mu$ must exist at this point since the adversary can win only with a Type-2 forgery). The challenger computes $\hat{S}=\prod_{\tau \in \mathcal{L}^{*}}\left(S_{\tau}\right)^{f_{\tau}^{*}}, \hat{R}=\prod_{\tau \in \mathcal{L}^{*}}\left(R_{\tau}\right)^{f_{\tau}^{*}}$ $\overrightarrow{\hat{m}}=\sum_{\tau \in \mathcal{L}^{*}} f_{\tau}^{*} \cdot \vec{m}_{\tau}$ where $\left\{R_{\tau}, S_{\tau}\right\}_{\tau}$ are the signature components generated by the challenger in all the signing queries $\left(\Delta_{\mu}, \tau, \vec{m}_{\tau}\right)$. If the forgery verifies correctly, i.e., $\operatorname{Ver}\left(\mathrm{vk}, \mathcal{P}_{\Delta^{*}}^{*}, \vec{m}^{*}, \sigma^{*}\right)=1$, and $\vec{m}^{*} \neq \overrightarrow{\hat{m}}$ and $S^{*}=\hat{S}$, then the challenger sets $\mathcal{A}_{3} \leftarrow$ true.

It is easy to see that $\operatorname{Pr}\left[G_{2}(\mathcal{A})\right]-\operatorname{Pr}\left[G_{3}(\mathcal{A})\right] \leq \operatorname{Pr}\left[\operatorname{Bad}_{3}\right]$. In Lemma 11 we show that any adversary for which $\operatorname{Bad}_{3}$ occurs can be reduced to a solver for the 1-DHI problem.

Game 4 This game proceeds as Game 3 except for the following change: at the beginning, the challenger chooses a random index $\mu \stackrel{\$}{\leftarrow}[Q]$, where $Q=\operatorname{poly}(\lambda)$ is the number of signing queries made by $\mathcal{A}$ during the game. Let $\Delta_{1}, \ldots, \Delta_{Q}$ be all the datasets queried by $\mathcal{A}$. Then if the dataset $\Delta^{*}$ used by $\mathcal{A}$ in the forgery is not $\Delta_{\mu}$, the challenger sets $\mathcal{A}_{4} \leftarrow$ true. As one can check, we have that $\operatorname{Pr}\left[G_{3}(\mathcal{A})\right]=Q \cdot \operatorname{Pr}\left[G_{4}(\mathcal{A})\right]$.

Game 5 proceeds as Game 4 except that at the end the challenger runs the following additional check: if $\operatorname{Ver}\left(\mathrm{vk}, \mathcal{P}_{\Delta^{*}}^{*}, \vec{m}^{*}, \sigma^{*}\right)=1$ and $\vec{m}^{*} \neq \overrightarrow{\hat{m}}$ and $S^{*} \neq \hat{S}$ and $R^{*}=\hat{R}$, then the challenger sets $\mathcal{A}_{5} \leftarrow$ true. It is easy to see that $\operatorname{Pr}\left[G_{4}(\mathcal{A})\right]-\operatorname{Pr}\left[G_{5}(\mathcal{A})\right] \leq \operatorname{Pr}\left[\operatorname{Bad}_{5}\right]$. In Lemma 13 we show that any 
Chapter 3. Improving Efficiency: Linearly Homomorphic Signatures with Sublinear Public Keys via APHFs

adversary for which $\mathrm{Bad}_{5}$ occurs can be reduced to a solver for the 2-DHI problem.

Game 6 proceeds as Game 5 with the following modification. At the very beginning, the challenger chooses the value $z_{\mu} \stackrel{\$}{\leftarrow} \mathbb{Z}_{p}$ that will be used to generate the signatures for $\mu$-th dataset $\Delta_{\mu}$. It sets $Z_{\mu}=g_{2}^{z_{\mu}}$. Second, instead of generating the key pek of the hash function $\mathrm{H}$ using $\mathrm{H}$.Gen, the challenger runs (td, pek) $\stackrel{\$}{\leftarrow}$ H.TrapGen $\left(1^{\lambda}\right.$, bgp, $\left.g_{1}, g_{1}, g_{2}, Z_{\mu}\right)$ where H.TrapGen is the algorithm for which $\mathrm{H}$ has $(1, \gamma, \epsilon)$-programmable pseudorandomness. Then the challenger uses td when it needs to compute $\mathrm{H}(\cdot)$ during the experiment.

If $\mathrm{H}$ hash $(1, \gamma, \epsilon)$-programmable pseudorandomness we immediately obtain that Game 5 and Game 6 are within statistical distance $\gamma$, i.e., $\mid \operatorname{Pr}\left[G_{5}(\mathcal{A})\right]-$ $\operatorname{Pr}\left[G_{6}(\mathcal{A})\right] \mid \leq \gamma$.

Game 7 This game is the same as Game 6, except that in the signing queries $(\Delta, \tau, \vec{m})$ such that $\Delta$ is the $\mu$-th distinct dataset queried by $\mathcal{A}$, the challenger first computes $\vec{c}_{\tau} \leftarrow \mathrm{H}$. TrapEval(td, $\left.\tau\right)$ and then generates the signature component $R_{\tau}$ by setting $R_{\tau}=g_{1}^{-c_{\tau, 0}}$, instead of choosing $R_{\tau} \stackrel{\$}{\leftarrow} \mathbb{G}_{1}$ randomly as done up to Game 6.

As we show in Lemma 14. Game 6 is computationally indistinguishable from Game 7 under the assumption that $\mathrm{H}$ has programmable pseudorandomness. Moreover, note that due to the previous modifications, Game 7 can output 1 only if the adversary outputs a forgery $\left(\mathcal{P}_{\Delta^{*}}^{*}, \sigma^{*}, \vec{m}^{*}\right)$ such that $\operatorname{Ver}(\mathrm{vk}$, $\left.\mathcal{P}_{\Delta^{*}}^{*}, \vec{m}^{*}, \sigma^{*}\right)=1$ and $\vec{m}^{*} \neq \overrightarrow{\hat{m}}$ and $S^{*} \neq \hat{S}$ and $R^{*} \neq \hat{R}$. We conclude the proof by showing in Lemma 15 that an adversary that wins in Game 7 can be used to solve the FDHI problem (Definition 11).

We proceed with the proof by formally bounding the difference between each consecutive pair of games, and eventually the probability that an adversary wins in the last game. The proof of Theorem 11 is finally obtained by putting together all the bounds.

Lemma 9. For every PPT $\mathcal{A}$ there exists a PPT forger $\mathbb{F}$ such that $\operatorname{Pr}\left[G_{0}(\mathcal{A})\right]-$ $\operatorname{Pr}\left[G_{1}(\mathcal{A})\right] \leq \mathbf{A d} \mathbf{v}_{\sigma^{\prime}, \mathbb{F}}^{\mathrm{UF}-\mathrm{CMA}}(\lambda)$.

Proof. The two games differ only if $\operatorname{Bad}_{1}$ occurs in Game 1, i.e., $\mid \operatorname{Pr}\left[G_{0}(\mathcal{A})\right]-$ $\operatorname{Pr}\left[G_{1}(\mathcal{A})\right] \mid \leq \operatorname{Pr}\left[\operatorname{Bad}_{1}\right]$. However, by the construction of HSig, if $\operatorname{Bad}_{1}$ occurs, it means that the forgery returned by $\mathcal{A}$ includes a valid signature $\sigma_{\Delta^{*}}$ on $\left(\Delta^{*} \mid Z^{*}\right)$ although no signature on $\left(\Delta^{*} \mid \cdot\right)$ was ever returned by the challenger during the experiment. It is straightforward to show that, for any such a PPT $\mathcal{A}$, there exists a PPT forger algorithm $\mathbb{F}$ that breaks the unforgeability of the regular signature scheme $\Sigma^{\prime}$, i.e., $\operatorname{Pr}\left[\operatorname{Bad}_{1}\right] \leq \operatorname{Adv}_{\sigma^{\prime}, \mathbb{F}}^{\mathrm{UF}-\mathrm{CMA}}(\lambda)$.

Lemma 10. For every PPT $\mathcal{A}$ there exists a PPT distinguisher $\mathcal{D}$ such that $\left|\operatorname{Pr}\left[G_{1}(\mathcal{A})\right]-\operatorname{Pr}\left[G_{2}(\mathcal{A})\right]\right| \leq \operatorname{Adv}_{F, \mathcal{D}}^{P R F}(\lambda)$. 
Chapter 3. Improving Efficiency: Linearly Homomorphic Signatures with Sublinear Public Keys via APHFs

Proof. Game 1 and Game 2 differ just for the fact that the PRF $F$ is replaced by a random function $\mathcal{R}$. It is easy to do a reduction to the security of the PRF to show that for any adversary $\mathcal{A}$ such that $\left|\operatorname{Pr}\left[G_{1}(\mathcal{A})\right]-\operatorname{Pr}\left[G_{2}(\mathcal{A})\right]\right| \geq \epsilon$ is non-negligible it is possible to construct a PPT distinguisher $\mathcal{D}$ that archives advantage $\epsilon$ against the pseudorandomness of $F$.

Lemma 11. If $\mathrm{H}$ is simply $(1, \gamma)$-programmable, and $\mathrm{H}^{\prime}$ is weakly (poly, $\left.1,2, \gamma^{\prime}, \delta^{\prime}\right)$ degree-2 programmable, then for every PPT $\mathcal{A}$ running in Game 3 there exists a PPT simulator $\mathcal{B}$ such that $\operatorname{Pr}\left[\operatorname{Bad}_{3}\right] \leq\left(T / \delta^{\prime}\right) \cdot \mathbf{A d v}_{\mathcal{B}}^{1-D H I}(\lambda)+\gamma+\gamma^{\prime}$.

Proof. Assume there exists a PPT adversary $\mathcal{A}$ such that $\operatorname{Pr}\left[\operatorname{Bad}_{3}\right] \geq \epsilon$. Then we show how to build a PPT simulator $\mathcal{B}$ that breaks the 1-DHI assumption with advantage greater than $\left(\delta^{\prime} \epsilon\right) / T-\gamma-\gamma^{\prime}$.

$\mathcal{B}$ takes as input a tuple $\left(g_{1}, g_{2}, g_{1}^{z}, g_{2}^{z}\right)$, and its goal is to compute $g_{1}^{z^{2}}$. Precisely, here we use the fact that this problem is equivalent to the 1-DHI problem in which the adversary has to compute $g_{1}^{1 / z}$. So, $\mathcal{B}$ proceeds as follows.

Setup: $\mathcal{B}$ starts by sampling a random $y \stackrel{\$}{\leftarrow} \mathbb{Z}_{p}$ and runs (td, pek $) \stackrel{\$}{\leftarrow} \mathrm{H}$. TrapGen $\left(1^{\lambda}\right.$, bgp, $\left.g_{1}, g_{1}, g_{2}, g_{2}^{y}\right)$. Note that since $\mathcal{B}$ had set $h_{1}=g_{1}$, the polynomials $c_{X}$ generated by $\mathrm{H}$.TrapEval(td, $X)$ will be univariate polynomials $c_{X}(y)$. Next, it chooses a random index $\nu \stackrel{\$}{\leftarrow}[T]$, which represents a guess on the index where the message vector $\vec{m}^{*}$ returned by the adversary in the forgery will differ from the "correct" result $\overrightarrow{\hat{m}}$. Then $\mathcal{B}$ sets $h_{1}=g_{1}^{z}, h_{2}=g_{2}^{z}$ and runs the trapdoor generation (for weakly degree-2 programmability) of the asymmetric hash function $\mathrm{H}^{\prime}-\left(\mathrm{td}^{\prime}\right.$, pek $) \stackrel{\$}{\leftarrow} \mathrm{H}^{\prime}$. TrapGen $\left(1^{\lambda}\right.$, bgp, $\left.g_{1}, h_{1}, g_{2}, h_{2}, \nu\right)-$ by providing $\nu$ as the input on which the coefficient $c_{\nu, 2} \neq 0$. Indeed, notice that by giving $h_{1}=g_{1}^{z}, h_{2}=g_{2}^{z}$ to $\mathrm{H}^{\prime}$. TrapGen, the polynomials generated $\mathrm{H}^{\prime}$. TrapEval $\left(\mathrm{td}^{\prime}, X\right)$ will be univariate polynomials $c_{X}(z)$.

Finally, the simulator generates the keys $\left(\mathbf{s k}^{\prime}, \mathrm{vk}^{\prime}\right)$ of the scheme $\Sigma^{\prime}$, sets $\mathrm{vk}=\left(\mathrm{vk}^{\prime}\right.$, pek, pek' $)$, stores $\mathrm{sk}^{\prime}, \mathrm{td}, \mathrm{td}^{\prime}$, and returns vk to $\mathcal{A}$.

Signing queries: Let $k \leftarrow 1$ be a counter for the number of datasets queried by $\mathcal{A}$. For every new queried dataset $\Delta, \mathcal{B}$ creates a list $T_{\Delta}$ of tuples $(\tau, \vec{m}, \sigma)$, which collects all the label/message pairs queried by the adversary on $\Delta$, and the respectively generated signatures. Moreover, whenever the $k$-th new dataset $\Delta_{k}$ is queried, $\mathcal{B}$ samples a random $\xi_{k} \stackrel{\$}{\leftarrow} \mathbb{Z}_{p}$, computes $Z_{k}=\left(g_{2}^{z}\right)^{\xi_{k}}$ and stores $\xi_{k}$. Note that all the values $\left\{Z_{k}\right\}_{k \in[Q]}$ are random in $\mathbb{G}_{2}$ and thus are distributed exactly as in Game 3.

Given a signing query $(\Delta, \tau, \vec{m})$ such that $\Delta=\Delta_{k}$ is the $k$-th dataset, $\mathcal{B}$ proceeds as follows. First, it runs $\vec{c}_{\tau} \leftarrow \mathrm{H}$. TrapEval $(\mathrm{td}, \tau)$, and $\vec{c}_{j}^{\prime} \leftarrow \mathrm{H}^{\prime}$.TrapEval $\left(\operatorname{td}^{\prime}, j\right)$ for all $j=1$ to $T$. If $c_{j, 2}^{\prime}=0$ then $\mathcal{B}$ continues the simulation as follows, otherwise it aborts. 
Chapter 3. Improving Efficiency: Linearly Homomorphic Signatures with Sublinear Public Keys via APHFs

Therefore, $\mathcal{B}$ samples a random $\rho_{\tau} \stackrel{\$}{\leftarrow} \mathbb{Z}_{p}$ and computes

$$
R_{\tau}=g_{1}^{-c_{\tau}(y)-\sum_{j=1}^{T} c_{j, 0}^{\prime} m_{j}} \cdot\left(g_{1}^{z}\right)^{\rho_{\tau}}, \quad S_{\tau}=\left(g_{1}^{\rho_{\tau}} \cdot g_{1}^{\sum_{j=1}^{T} c_{j, 1}^{\prime} m_{j}} \cdot\left(g_{1}^{z}\right)^{c_{\nu, 2}^{\prime} m_{\nu}}\right)^{\frac{1}{\xi_{k}}}
$$

As one can see, the value $R_{\tau}$ is a uniformly distributed $\mathbb{G}_{1}$ element as in Game 3. Moreover, $S_{\tau}$ is a correctly distributed signature since

$$
\begin{aligned}
S_{\tau} & =\left(g_{1}^{\rho_{\tau}} \cdot g_{1}^{\sum_{j=1}^{T} c_{j, 1}^{\prime} m_{j}} \cdot\left(g_{1}^{z}\right)^{c_{\nu, 2}^{\prime} m_{\nu}}\right)^{\frac{1}{\xi_{k}}}=\left(g_{1}^{z \rho_{\tau}} \cdot g_{1}^{\sum_{j=1}^{T} z c_{j, 1}^{\prime} m_{j}} \cdot\left(g_{1}^{z^{2}}\right)^{c_{\nu, 2}^{\prime} m_{\nu}}\right)^{\frac{1}{z_{k}}} \\
& =\left(g_{1}^{c_{\tau}(y)} \cdot g_{1}^{-c_{\tau}(y)-\sum_{j=1}^{T} c_{j, 0}^{\prime} m_{j}} \cdot\left(g_{1}^{z}\right)^{\rho_{\tau}} \cdot g_{1}^{\sum_{j=1}^{T} c_{j, 0}^{\prime} m_{j}} \cdot g_{1}^{\sum_{j=1}^{T} z c_{j, 1}^{\prime} m_{j}} \cdot\left(g_{1}^{z^{2}}\right)^{c_{\nu, 2}^{\prime} m_{\nu}}\right)^{\frac{1}{z_{k}}} \\
& =\left(\mathrm{H}(\tau) \cdot R_{\tau} \cdot g_{1}^{\sum_{j=1}^{T}\left(c_{j, 0}^{\prime}+c_{j, 1}^{\prime} z+c_{j, 2}^{\prime} z^{2}\right) m_{j}}\right)^{\frac{1}{z_{k}}} \\
& =\left(\mathrm{H}(\tau) \cdot R_{\tau} \cdot \prod_{j=1}^{T} g_{1}^{c_{j}^{\prime}(z) m_{j}}\right)^{\frac{1}{z_{k}}}=\left(\mathrm{H}(\tau) \cdot R_{\tau} \cdot \prod_{j=1}^{T} \mathrm{H}^{\prime}(j)^{m_{j}}\right)^{\frac{1}{z_{k}}}
\end{aligned}
$$

Finally, $\mathcal{B}$ returns to $\mathcal{A}$ the signature $\sigma=\left(\sigma_{\Delta}, Z_{k}, R_{\tau}, S_{\tau}\right)$, where $\sigma_{\Delta} \stackrel{\$}{\leftarrow}$ $\operatorname{Sign}\left(\mathrm{sk}^{\prime}, \Delta \mid Z_{k}\right)$.

Forgery: Let $\left(\mathcal{P}_{\Delta^{*}}^{*}, \sigma^{*}, \vec{m}^{*}\right)$ be the forgery returned by the adversary. $\mathcal{B}$ proceeds exactly as the challenger in Game 3 in order to compute $\hat{R}, \hat{S}, \overrightarrow{\hat{m}}$. If $\operatorname{Bad}_{3}$ occurs, since $\left(\mathcal{P}_{\Delta^{*}}^{*}, \sigma^{*}, \vec{m}^{*}\right)$ verifies correctly the following two equations hold

$$
\begin{gathered}
e\left(S^{*}, Z_{\mu}\right)=\Lambda \cdot e\left(R^{*}, g_{2}\right) \cdot \prod_{j=1}^{T} \text { H.PubEval }\left(\text { pek }^{\prime}, j\right)^{m_{j}^{*}}, \\
e\left(\hat{S}, Z_{\mu}\right)=\Lambda \cdot e\left(\hat{R}, g_{2}\right) \cdot \prod_{j=1}^{T} \text { H.PubEval' }\left(\text { pek }^{\prime}, j\right)^{\hat{m}_{j}}
\end{gathered}
$$

where $\Lambda=\prod_{\tau \in \mathcal{L}^{*}}$ H.PubEval(pek, $\left.\tau\right)^{f_{\tau}^{*}}$. If we divide the two equations and consider that, by definition of $\operatorname{Bad}_{3}$, it holds $S^{*}=\hat{S}$, then we obtain

$$
\frac{\hat{R}}{R^{*}}=\prod_{j=1}^{T} \mathrm{H}^{\prime}(j)^{m_{j}^{*}-\hat{m}_{j}}
$$

By correctness of the trapdoor algorithms of $\mathrm{H}^{\prime}$ we know that $\mathrm{H}^{\prime}(j)=$ $g_{1}^{c_{j, 0}^{\prime}+c_{j, 1}^{\prime} z+c_{j, 2}^{\prime} z^{2}}$ where $c_{j, 2}^{\prime}=0$ for all $j \neq \nu$ (since $\mathcal{B}$ did not abort so far). If $c_{\nu, 2}^{\prime}=0$ then $\mathcal{B}$ aborts, otherwise it continues as follows. However, notice that by the weak (poly, $1,2, \gamma^{\prime}, \delta^{\prime}$ ) programmability of $\mathrm{H}^{\prime}$ the event that 
Chapter 3. Improving Efficiency: Linearly Homomorphic Signatures with Sublinear Public Keys via APHFs

$c_{j, 2}^{\prime}=0$ for all $j \neq \nu$ and $c_{\nu, 2}^{\prime} \neq 0$ holds with probability $\delta^{\prime}$. Therefore, $\mathcal{B}$ does not abort with probability $\delta^{\prime}$. Furthermore, since the simulation provided to $\mathcal{A}$ until its forgery's output is distributed statistically close to the real execution of Game 3 (close by a factor $\gamma+\gamma^{\prime}$ due to the use of TrapGen in $\mathrm{H}$ and $\mathrm{H}^{\prime}$ ), $\nu$ is information-theoretically hidden to $\mathcal{A}$. Hence,

$$
\frac{\hat{R}}{R^{*}}=g_{1}^{\sum_{j=1}^{T}\left(c_{j, 0}^{\prime}+c_{j, 1}^{\prime} z+c_{j, 2}^{\prime} z^{2}\right)\left(m_{j}^{*}-\hat{m}_{j}\right)}=g_{1}^{\sum_{j=1}^{T}\left(c_{j, 0}^{\prime}+c_{j, 1}^{\prime} z\right)\left(m_{j}^{*}-\hat{m}_{j}\right)+c_{\nu, 2}^{\prime} z^{2}\left(m_{\nu}^{*}-\hat{m}_{\nu}\right)}
$$

Since $\vec{m}^{*} \neq \overrightarrow{\hat{m}}$ there must exist an index $\nu^{\prime} \in[T]$ such that $m_{\nu^{\prime}}^{*} \neq \hat{m}_{\nu^{\prime}}$. If $\nu^{\prime} \neq \nu$ then $\mathcal{B}$ aborts, otherwise it computes

$$
g_{1}^{z^{2}}=\left(\frac{\hat{R} \cdot g_{1}^{-\sum_{j=1}^{T}\left(c_{j, 0}^{\prime}+c_{j, 1}^{\prime} z\right)\left(m_{j}^{*}-\hat{m}_{j}\right)}}{R^{*}}\right)^{\frac{1}{c_{\nu, 2}^{\prime}\left(m_{\nu}^{*}-\hat{m}_{\nu}\right)}}
$$

It is easy to see that if $\mathcal{B}$ does not abort, $\mathcal{B}$ is able to compute the solution $g_{1}^{z^{2}}$ of the 1 -DHI problem. The probability that $\mathcal{B}$ does not abort is $\delta^{\prime} \cdot \operatorname{Pr}\left[\nu^{\prime}=\right.$ $\nu]=\delta^{\prime} / T$ since $\nu$ is uniformly distributed and completely hidden from the view of $\mathcal{A}$. In conclusion, we have that if $\operatorname{Pr}\left[\mathrm{Bad}_{3}\right] \geq \epsilon$ then $\mathcal{B}$ has advantage at least $\left(\delta^{\prime} \epsilon\right) / T-\gamma-\gamma^{\prime}$.

Lemma 12. $\operatorname{Pr}\left[G_{3}(\mathcal{A})\right]=Q \cdot \operatorname{Pr}\left[G_{4}(\mathcal{A})\right]$

Proof. First, note that $\operatorname{Pr}\left[G_{4}(\mathcal{A})\right]=\operatorname{Pr}\left[G_{4}(\mathcal{A}) \wedge \operatorname{Bad}_{4}\right]+\operatorname{Pr}\left[G_{4}(\mathcal{A}) \wedge \neg \operatorname{Bad}_{4}\right]=$ $\operatorname{Pr}\left[G_{4}(\mathcal{A}) \mid \neg \operatorname{Bad}_{4}\right] \operatorname{Pr}\left[\neg \operatorname{Bad}_{4}\right]$ since Game 4 outputs 0 whenever $\operatorname{Bad}_{4}$ occurs. Second, observe that when $\mathrm{Bad}_{4}$ does not occur (i.e., the challenger guesses correctly the query index $\mu$ of the dataset $\left.\Delta^{*}\right)$ then the outcome of Game 4 is identical to the one of Game 3, i.e., $\operatorname{Pr}\left[G_{4}(\mathcal{A}) \mid \neg \operatorname{Bad}_{4}\right]=\operatorname{Pr}\left[G_{3}(\mathcal{A})\right]$. Since $\mu$ is chosen uniformly at random and is completely hidden to $\mathcal{A}$ we have that $\operatorname{Pr}\left[\neg \operatorname{Bad}_{4}\right]=1 / Q$, from which the lemma follows.

Lemma 13. If $\mathrm{H}$ is simply $(1, \gamma)$-programmable, and $\mathrm{H}^{\prime}$ is weakly (poly, $\left.1,2, \gamma^{\prime}, \delta^{\prime}\right)$ programmable, then for every PPT $\mathcal{A}$ running in Game 5 there exists a PPT simulator $\mathcal{B}$ such that $\operatorname{Pr}\left[\operatorname{Bad}_{5}\right] \leq\left(T / \delta^{\prime}\right) \cdot \mathbf{A d v}_{\mathcal{B}}^{2-D H I}(\lambda)+\gamma+\gamma^{\prime}$.

Proof. Assume there exists a PPT adversary $\mathcal{A}$ such that $\operatorname{Pr}\left[\mathrm{Bad}_{5}\right] \geq \epsilon$. Then we show how to build a PPT simulator $\mathcal{B}$ that breaks the 2 -DHI assumption in $\mathbb{G}_{1}$ with advantage greater than $\left(\delta^{\prime} \epsilon\right) / T-\gamma-\gamma^{\prime}$.

$\mathcal{B}$ takes as input a tuple $\left(g_{1}, g_{2}, g_{1}^{z}, g_{2}^{z}, g_{1}^{z^{2}}, g_{2}^{z^{2}}\right)$, and its goal is to compute $g_{1}^{1 / z}$. To do so $\mathcal{B}$ proceeds as follows.

Setup: $\mathcal{B}$ proceeds as the challenger in Game 5 by choosing a random index $\mu \stackrel{\$}{\leftarrow}[Q]$. Second, $\mathcal{B}$ picks a random $y \stackrel{\$}{\leftarrow} \mathbb{Z}_{p}$ and runs (td, pek) $\stackrel{\$}{\leftarrow}$ H.TrapGen $\left(1^{\lambda}\right.$, bgp, $\left.g_{1}, g_{1}, g_{2}, g_{2}^{y}\right)$. Note that since $\mathcal{B}$ had set $h_{1}=g_{1}$, the 
Chapter 3. Improving Efficiency: Linearly Homomorphic Signatures with Sublinear Public Keys via APHFs

polynomials $c_{X}$ generated by $\mathrm{H}$.TrapEval(td, $\left.X\right)$ will be univariate, degree1 , polynomials $c_{X}(y)$. Next, it chooses a random index $\nu \stackrel{\$}{\leftarrow}[T]$, which represents a guess on the index where the message vector $\vec{m}^{*}$ returned by the adversary in the forgery will differ from the "correct" result $\overrightarrow{\hat{m}}$. It runs the trapdoor generation (for weak (poly, 1,2)-programmability) of the asymmetric hash function $\mathrm{H}^{\prime}-\left(\mathrm{td}^{\prime}, \operatorname{pek}^{\prime}\right) \stackrel{\$}{\leftarrow} \mathrm{H}^{\prime}$.TrapGen $\left(1^{\lambda}\right.$, bgp $\left., g_{1}, g_{1}^{z}, g_{2}, g_{2}^{z}, \nu\right)-$ providing $\nu$ as the input on which the coefficient $c_{\nu, 0} \neq 0$. Notice that by giving $h_{1}=g_{1}^{z}, h_{2}=g_{2}^{z}$ to $\mathrm{H}^{\prime}$.TrapGen, the polynomials generated $\mathrm{H}^{\prime}$.TrapEval $\left(\mathrm{td}^{\prime}, X\right)$ will be univariate polynomials $c_{X}(z)=c_{X, 0}+c_{X, 1} z+$ $c_{X, 2} z^{2}$.

Finally, it generates the keys $\left(\mathbf{s k}^{\prime}, \mathrm{vk}^{\prime}\right)$ of the scheme $\Sigma^{\prime}$, sets $v \mathrm{k}=\left(\mathrm{vk}^{\prime}\right.$, pek, pek'), stores $\mathrm{sk}^{\prime}, \mathrm{td}, \mathrm{td}^{\prime}$, and returns vk to $\mathcal{A}$.

Signing queries: Let $k \leftarrow 1$ be a counter for the number of datasets queried by $\mathcal{A}$. For every new queried dataset $\Delta, \mathcal{B}$ creates a list $T_{\Delta}$ of tuples $(\tau, \vec{m}, \sigma)$, which collects all the label/message pairs queried by the adversary on $\Delta$ and the respectively generated signatures. Moreover, whenever the $k$-th new dataset $\Delta_{k}$ is queried, $\mathcal{B}$ does the following: if $k=\mu$ it samples a random $\xi \stackrel{\$}{\leftarrow} \mathbb{Z}_{p}$, computes $Z_{\mu}=\left(g_{2}^{z}\right)^{\xi}$ and stores $\xi$; if $k \neq \mu, \mathcal{B}$ samples directly a random $z_{k} \stackrel{\$}{\leftarrow} \mathbb{Z}_{p}$, computes $Z_{k}=g_{2}^{z_{k}}$ and stores $z_{k}$. Note that all the values $\left\{Z_{k}\right\}_{k \in[Q]}$ are random in $\mathbb{G}_{2}$ and thus are distributed exactly as in Game 5.

Given a signing query $(\Delta, \tau, \vec{m})$ such that $\Delta=\Delta_{k}$ is the $k$-th dataset, $\mathcal{B}$ first computes $\sigma_{\Delta_{k}} \leftarrow \operatorname{Sign}\left(\mathrm{sk}^{\prime}, \Delta_{k}, Z_{k}\right)$, and then proceeds as follows.

- If $k \neq \mu, \mathcal{B}$ runs $\vec{c}_{\tau} \leftarrow \mathrm{H}$.TrapEval $(\mathrm{td}, \tau)$, and $\vec{c}_{j}^{\prime} \leftarrow \mathrm{H}^{\prime}$.TrapEval $\left(\mathrm{td}^{\prime}, j\right)$ for all $j=1$ to $T$. It samples $R_{\tau} \stackrel{\$}{\leftarrow} \mathbb{G}_{1}$, and computes

$$
S_{\tau}=\left(g_{1}^{c_{\tau}(y)} \cdot R_{\tau} \cdot \prod_{j=1}^{T} g_{1}^{c_{j}^{\prime}(z) m_{j}}\right)^{\frac{1}{z_{k}}}
$$

In particular, note that every $g_{1}^{c_{j}^{\prime}(z)}$ can be computed by $\mathcal{B}$ using the values $g_{1}^{z}, g_{1}^{z^{2}}$.

- If $k=\mu, \mathcal{B}$ runs $\vec{c}_{\tau} \leftarrow \mathrm{H}$.TrapEval $(\mathrm{td}, \tau)$, and $\vec{c}_{j}^{\prime} \leftarrow \mathrm{H}^{\prime}$.TrapEval $\left(\mathrm{td}^{\prime}, j\right)$ for all $j=1$ to $T$. If $c_{j, 0}^{\prime}=0$ for all $j \neq \nu$, then $\mathcal{B}$ continues the simulation as follows, otherwise it aborts.

Therefore, $\mathcal{B}$ samples a random $\rho_{\tau} \stackrel{\$}{\leftarrow} \mathbb{Z}_{p}$ and computes

$$
R_{\tau}=g_{1}^{-c_{\tau}(y)-c_{\nu, 0} m_{\nu}} \cdot\left(g_{1}^{z}\right)^{\rho_{\tau}}, \quad S_{\tau}=\left(g_{1}^{\rho_{\tau}} \cdot g_{1}^{\sum_{j=1}^{T}\left(c_{j, 1}^{\prime}+c_{j, 2}^{\prime} z\right) m_{j}}\right)^{\frac{1}{\xi}}
$$


Chapter 3. Improving Efficiency: Linearly Homomorphic Signatures with Sublinear Public Keys via APHFs

As one can see, the value $R_{\tau}$ is a uniformly distributed $\mathbb{G}_{1}$ element as in Game 5. Moreover, $S_{\tau}$ is a correctly distributed signature since

$$
\begin{aligned}
S_{\tau} & =\left(g_{1}^{\rho_{\tau}} \cdot g_{1}^{\sum_{j=1}^{T}\left(c_{j, 1}^{\prime}+c_{j, 2}^{\prime} z\right) m_{j}}\right)^{\frac{1}{\xi}}=\left(g_{1}^{z \rho_{\tau}} \cdot g_{1}^{\sum_{j=1}^{T}\left(c_{j, 1}^{\prime} z+c_{j, 2}^{\prime} z^{2}\right) m_{j}}\right)^{\frac{1}{z \xi}} \\
& =\left(g_{1}^{c_{\tau}(y)} \cdot g_{1}^{-c_{\tau}(y)-c_{\nu, 0}^{\prime} m_{\nu}} \cdot g_{1}^{z \rho_{\tau}} \cdot g_{1}^{\sum_{j=1}^{T}\left(c_{j, 0}^{\prime}+z c_{j, 1}^{\prime}+c_{j, 2}^{\prime} z^{2}\right) m_{j}}\right)^{\frac{1}{z \xi}} \\
& =\left(\mathrm{H}(\tau) \cdot R_{\tau} \cdot g_{1}^{\sum_{j=1}^{T}\left(c_{j, 0}^{\prime}+z c_{j, 1}^{\prime}+c_{j, 2}^{\prime} z^{2}\right) m_{j}}\right)^{\frac{1}{z \xi}} \\
& =\left(\mathrm{H}(\tau) \cdot R_{\tau} \cdot \prod_{j=1}^{T} \mathrm{H}^{\prime}(j)^{m_{j}}\right)^{\frac{1}{z_{\mu}}}
\end{aligned}
$$

Finally, $\mathcal{B}$ returns to $\mathcal{A}$ the signature $\sigma=\left(\sigma_{\Delta_{k}}, Z_{k}, R_{\tau}, S_{\tau}\right)$.

Forgery: Let $\left(\mathcal{P}_{\Delta^{*}}^{*}, \sigma^{*}, \vec{m}^{*}\right)$ be the forgery returned by the adversary. $\mathcal{B}$ proceeds exactly as the challenger in Game 5 in order to compute $\hat{R}, \hat{S}, \overrightarrow{\hat{m}}$. If $\operatorname{Bad}_{5}$ occurs, since $\left(\mathcal{P}_{\Delta^{*}}^{*}, \sigma^{*}, \vec{m}^{*}\right)$ verifies correctly the following two equations hold

$$
\begin{gathered}
e\left(S^{*}, Z_{\mu}\right)=\Lambda \cdot e\left(R^{*}, g_{2}\right) \cdot \prod_{j=1}^{T} \text { H.PubEval' }\left(\text { pek }^{\prime}, j\right)^{m_{j}^{*}}, \\
e\left(\hat{S}, Z_{\mu}\right)=\Lambda \cdot e\left(\hat{R}, g_{2}\right) \cdot \prod_{j=1}^{T} \text { H.PubEval }\left(\text { pek }^{\prime}, j\right)^{\hat{m}_{j}}
\end{gathered}
$$

where $\Lambda=\prod_{\tau \in \mathcal{L}^{*}}$ H.PubEval(pek, $\left.\tau\right)^{f_{\tau}^{*}}$. If we divide the two equations and consider that by definition of $\operatorname{Bad}_{3}, S^{*} \neq \hat{S}$ but $R^{*}=\hat{R}$ we obtain

$$
\frac{S^{*}}{\hat{S}}=\left(\prod_{j=1}^{T} \mathrm{H}^{\prime}(j)^{m_{j}^{*}-\hat{m}_{j}}\right)^{\frac{1}{z \xi}}
$$

By using the $\mathrm{H}^{\prime}$.TrapEval algorithm we know that $\mathrm{H}^{\prime}(j)=g_{1}^{c_{j, 0}^{\prime}+z c_{j, 1}^{\prime}+c_{j, 2}^{\prime} z^{2}}$ where $c_{j, 0}^{\prime}=0$ for all $j \neq \nu$ (since $\mathcal{B}$ did not abort so far). If $c_{\nu, 0}^{\prime}=0$, then $\mathcal{B}$ aborts, otherwise it continues as follows. Nevertheless, notice that by weak (poly, 1,2)-programmability of $\mathrm{H}^{\prime}, c_{j, 0}^{\prime}=0$ for all $j \neq \nu$, whereas $c_{\nu, 0}^{\prime} \neq 0$ holds with probability $\delta^{\prime}$, which means that with probability at least $\delta^{\prime} \mathcal{B}$ does not abort. Hence,

$$
\begin{aligned}
& \frac{S^{*}}{\hat{S}}=\left(g_{1}^{1 /(z \xi)}\right)^{\sum_{j=1}^{T}\left(c_{j, 0}^{\prime}+z c_{j, 1}^{\prime}+c_{j, 2}^{\prime} z^{2}\right)\left(m_{j}^{*}-\hat{m}_{j}\right)} \\
= & \left(g_{1}^{1 /(z \xi)}\right)^{c_{\nu, 0}^{\prime}\left(m_{\nu}^{*}-\hat{m}_{\nu}\right)} g_{1}^{\sum_{j=1}^{T}\left(c_{j, 1}^{\prime}+c_{j, 2}^{\prime} z\right)\left(m_{j}^{*}-\hat{m}_{j}\right) / \xi}
\end{aligned}
$$


Chapter 3. Improving Efficiency: Linearly Homomorphic Signatures with Sublinear Public Keys via APHFs

Since $\vec{m}^{*} \neq \overrightarrow{\hat{m}}$ there must exist an index $\nu^{\prime} \in[T]$ such that $m_{\nu^{\prime}}^{*} \neq \hat{m}_{\nu^{\prime}}$. If $\nu^{\prime} \neq \nu$ then $\mathcal{B}$ aborts, otherwise it computes

$$
g_{1}^{1 / z}=\left(\frac{S^{*} \cdot g_{1}^{-\sum_{j=1}^{T}\left(c_{j, 1}^{\prime}+c_{j, 2}^{\prime} z\right)\left(m_{j}^{*}-\hat{m}_{j}\right) / \xi}}{\hat{S}}\right)^{\frac{\xi}{c_{\nu, 0}^{\prime}\left(m_{\nu}^{*}-\hat{m}_{\nu}\right)}}
$$

Note that the simulation of Game 5 provided by $\mathcal{B}$ to $\mathcal{A}$ is statistically close (by a factor $\gamma+\gamma^{\prime}$ due to the use of TrapGen in $\mathrm{H}_{\text {and }} \mathrm{H}^{\prime}$ ) to the real execution of Game 5. Then, it is easy to see that if $\mathcal{B}$ does not abort, it is able to compute the solution of the 2-DHI problem $g_{1}^{1 / z}$. The probability that $\mathcal{B}$ does not abort is $\delta^{\prime} \cdot \operatorname{Pr}\left[\nu^{\prime}=\nu\right]=\delta^{\prime} / T$ since $\nu$ is uniformly distributed and completely hidden from the view of $\mathcal{A}$. In conclusion, we have that if $\operatorname{Pr}\left[\operatorname{Bad}_{5}\right] \geq \epsilon$ then $\mathcal{B}$ has advantage at least $\left(\delta^{\prime} \epsilon\right) / T-\gamma-\gamma^{\prime}$.

Lemma 14. If the asymmetric hash function $\mathrm{H}$ has $(1, \gamma, \epsilon)$-programmable pseudorandomness then $\left|\operatorname{Pr}\left[G_{6}(\mathcal{A})\right]-\operatorname{Pr}\left[G_{7}(\mathcal{A})\right]\right| \leq \epsilon$.

Proof. We do the proof by contradiction. Assume there exists a PPT adversary $\mathcal{A}$ such that $\left|\operatorname{Pr}\left[G_{1}\right]-\operatorname{Pr}\left[G_{2}\right]\right| \geq \epsilon$. Then we show how to build a PPT simulator $\mathcal{B}$ that breaks the programmable pseudorandomness of $\mathrm{H}$ with advantage $\epsilon$. We build such a simulator $\mathcal{B}$ as follows:

Setup: $\mathcal{B}$ first receives the bilinear group parameters bgp, which includes the two generators $g_{1}, g_{2}$. $\mathcal{B}$ proceeds as the challenger in Game 6 by choosing a random index $\mu \stackrel{\$}{\leftarrow}[Q]$ and a random $z_{\mu} \stackrel{\$}{\leftarrow} \mathbb{Z}_{p}$. It also prepares $Z_{\mu}=g_{2}^{z_{\mu}}$. Then it sets $h_{1}=g_{1} \in \mathbb{G}_{1}, h_{2}=Z_{\mu}$ and returns $\left(h_{1}, h_{2}\right)$ to its challenger. It receives back a public key pek for $\mathrm{H}$, and also gets access to an oracle that on input $\tau$ outputs $\mathrm{H}(\tau)$ and either $g_{1}^{c_{\tau, 0}}$ or $g_{1}^{r_{\tau}}$.

$\mathcal{B}$ then queries its oracle on all inputs $\tau \in[N]$ and stores all the answers $\left\{Y_{\tau}, C_{\tau}\right\}_{\tau \in[N]}$. Moreover, $\mathcal{B}$ chooses in advance the values $z_{k} \stackrel{\$}{\leftarrow} \mathbb{Z}_{p}, \forall k \in$ $[Q] \backslash\{\mu\}$, and stores $\left\{z_{k}, Z_{k}=g_{2}^{z_{k}}\right\}$. Next, it generates the keys (sk', $\mathbf{v k}^{\prime}$ ) of the scheme $\Sigma^{\prime}$, the keys $\left(\right.$ sek $^{\prime}$, pek') of the asymmetric hash $\mathrm{H}^{\prime}$, it sets $\mathrm{vk}=\left(\mathrm{vk}^{\prime}\right.$, pek, pek' $)$, stores $\mathrm{sk}^{\prime}, \mathrm{sek}^{\prime}$, and returns vk to $\mathcal{A}$.

Signing queries: Let $k \leftarrow 1$ be a counter for the number of datasets queried by $\mathcal{A}$. For every new queried dataset $\Delta, \mathcal{B}$ creates a list $T_{\Delta}$ of tuples $(\tau, \vec{m}, \sigma)$, which collects all the label/message pairs queried by the adversary on $\Delta$ and the respectively generated signatures.

On the $k$-th query $(\Delta, \tau, \vec{m}), \mathcal{B}$ proceeds as follows:

- If $k \neq \mu, \mathcal{B}$ first generates the signature $\sigma_{\Delta}$ on $\left(\Delta_{k}, Z_{k}\right)$ using the secret key sk'. Next, it chooses a random value $R_{\tau} \stackrel{\$}{\leftarrow} \mathbb{G}_{1}$ and computes $S_{\tau}=\left(Y_{\tau} \cdot R_{\tau} \prod_{j=1}^{T} \text { H.PriEval' }\left(\text { sek }^{\prime}, j\right)^{m_{j}}\right)^{1 / z_{k}}$. 
Chapter 3. Improving Efficiency: Linearly Homomorphic Signatures with Sublinear Public Keys via APHFs

- If $k=\mu, \mathcal{B}$ works exactly as above except that it sets $R_{\tau}=C_{\tau}^{-1}$.

Finally, $\mathcal{B}$ returns to $\mathcal{A}$ the signature $\sigma=\left(\sigma_{\Delta}, Z_{k}, R_{\tau}, S_{\tau}\right)$, where $\sigma_{\Delta} \stackrel{\$}{\leftarrow}$ $\operatorname{Sign}\left(\mathrm{sk}^{\prime}, \Delta \mid Z_{k}\right)$.

Forgery: Let $\left(\mathcal{P}_{\Delta^{*}}^{*}, \sigma^{*}, \vec{m}^{*}\right)$ be the forgery returned by the adversary. $\mathcal{B}$ proceeds exactly as the challenger in Game 7 in order to determine the outcome of the experiment, and outputs 0 or 1 accordingly.

It is easy to see that if $\mathcal{B}$ receives from its oracle values $C_{\tau}$ with the pseudorandom distribution, then $\mathcal{B}$ is perfectly simulating Game 7 to $\mathcal{A}$. Otherwise, if the values $C_{\tau}$ are random then $\mathcal{B}$ is simulating Game 6 . Therefore,

$$
\left|\operatorname{Pr}\left[\operatorname{Exp}_{\mathcal{B}, \mathrm{H}}^{P R H-0}=1\right]-\operatorname{Pr}\left[\operatorname{Exp}_{\mathcal{B}, \mathrm{H}}^{P R H-1}=1\right]\right|=\left|\operatorname{Pr}\left[G_{7}(\mathcal{A})\right]-\operatorname{Pr}\left[G_{6}(\mathcal{A})\right]\right|=\epsilon
$$

To conclude the proof, we are left with showing that any PPT adversary has negligible probability of winning in Game 7 . We show this in the following lemma where we prove that this holds under the Flexible Diffie-Hellman Inversion Assumption (FDHI) given in Definition 11.

Lemma 15. If $\mathrm{H}$ has $(1, \gamma, \epsilon)$-programmable pseudorandomness and $\mathrm{H}^{\prime}$ is (poly, $\left.0,1, \gamma^{\prime}, \delta^{\prime}\right)$-programmable, then for any PPT $\mathcal{A}$ running in Game 7 there is a PPT $\mathcal{B}$ against the FDHI assumption such that $\operatorname{Pr}\left[G_{7}(\mathcal{A})\right]=\mathbf{A d v}_{\mathcal{B}}^{F D H I}(\lambda) / \delta^{\prime}+\gamma+\gamma^{\prime}$.

Proof. Assume that $\mathcal{A}$ is a PPT adversary such that $\operatorname{Pr}\left[G_{7}(\mathcal{A})\right]=\epsilon$. Then we show how to build a PPT simulator $\mathcal{B}$ which uses $\mathcal{A}$ to solve the FDHI problem with advantage $\epsilon$. $\mathcal{B}$ receives an FDHI instance $\left(g_{1}, g_{2}, g_{2}^{z}, g_{2}^{v}, g_{1}^{\frac{z}{v}}, g_{1}^{r}, g_{1}^{\frac{r}{v}}\right)$ and works as follows.

Setup: $\mathcal{B}$ proceeds as the challenger in Game 6 by choosing a random index $\mu \stackrel{\$}{\leftarrow}$ $[Q]$. Next, it runs the trapdoor generation algorithm for the programmable pseudorandomness of $\mathrm{H}$, (td, pek) $\stackrel{\$}{\leftarrow} \mathrm{H}$. TrapGen $\left(1^{\lambda}\right.$, bgp, $\left.g_{1}, g_{1}, g_{2}, g_{2}^{z}\right)$, and the trapdoor generation algorithm for the (poly, 0,1)-programmability of $\mathrm{H}^{\prime},\left(\mathrm{td}^{\prime}\right.$, pek $) \stackrel{\$}{\leftarrow} \mathrm{H}^{\prime}$.TrapGen $\left(1^{\lambda}\right.$, bgp $\left., g_{1}, g_{1}, g_{2}, g_{2}^{z}\right)$.

Finally, it generates the keys $\left(\mathrm{sk}^{\prime}, \mathrm{vk}^{\prime}\right)$ of the scheme $\Sigma^{\prime}$, sets $v \mathrm{k}=\left(\mathrm{vk}^{\prime}\right.$, pek, pek $^{\prime}$, , stores $\mathrm{sk}^{\prime}, \mathrm{td}, \mathrm{td}^{\prime}$, and returns vk to $\mathcal{A}$.

Signing queries: Let $k \leftarrow 1$ be a counter for the number of datasets queried by $\mathcal{A}$. For every new queried dataset $\Delta, \mathcal{B}$ creates a list $T_{\Delta}$ of tuples $(\tau, \vec{m}, \sigma)$, which collects all the label/message pairs queried by the adversary on $\Delta$ and the respectively generated signatures.

Moreover whenever the $k$-th new dataset $\Delta_{k}$ is queried, $\mathcal{B}$ does the following: if $k=\mu$ it samples a random $\xi_{\mu} \stackrel{\$}{\leftarrow} \mathbb{Z}_{p}$, computes $Z_{\mu}=\left(g_{2}^{z}\right)^{\xi_{\mu}}$ and stores 
Chapter 3. Improving Efficiency: Linearly Homomorphic Signatures with Sublinear Public Keys via APHFs

$Z_{\mu}, \xi_{\mu}$; if $k \neq \mu, \mathcal{B}$ samples a random $\xi_{k} \stackrel{\$}{\leftarrow} \mathbb{Z}_{p}$, and computes $Z_{k}=\left(g_{2}^{v}\right)^{\xi_{k}}$ and stores $Z_{k}, \xi_{k}$. Note that all the values $\left\{Z_{k}\right\}_{k \in[Q]}$ are random in $\mathbb{G}_{2}$ and thus are distributed exactly as in Game 7.

Given a signing query $(\Delta, \tau, \vec{m})$ such that $\Delta=\Delta_{k}$ is the $k$-th dataset, $\mathcal{B}$ first computes $\sigma_{\Delta_{k}} \leftarrow \operatorname{Sign}\left(\mathrm{sk}^{\prime}, \Delta_{k}, Z_{k}\right)$, and then proceeds as follows.

- If $k \neq \mu$ : $\mathcal{B}$ runs $\vec{c}_{\tau} \leftarrow \mathrm{H}$.TrapEval(td, $\left.\tau\right)$, and $\vec{c}_{j}^{\prime} \leftarrow \mathrm{H}^{\prime}$.TrapEval $\left(\mathrm{td}^{\prime}, j\right)$ for all $j=1$ to $T$. Notice that by the $(1, \gamma, \epsilon)$-programmability of $\mathrm{H}$ we have that $\vec{c}_{\tau}$ is a degree-1 polynomial in $z: c_{\tau}(z)=c_{\tau, 0}+c_{\tau, 1} z$. Similarly, the polynomials $\vec{c}_{j}^{\prime}$ generated by $\mathrm{H}^{\prime}$.TrapEval are also of degree 1 in the sole variable $z$; If $c_{j, 0}^{\prime}=0$, then $\mathcal{B}$ continues the simulation as follows, otherwise it aborts. Notice that, by (poly, 0, 1)-programmability of $\mathrm{H}^{\prime}$, the event that $\forall j \in[T]: c_{j, 0}^{\prime}=0$, i.e., $c_{j}^{\prime}(z)=c_{j, 1}^{\prime} z$ holds with probability at least $\delta^{\prime}$. Therefore, $\mathcal{B}$ does not abort with probability at least $\delta^{\prime}$.

Next, it samples $\rho_{\tau} \stackrel{\$}{\leftarrow} \mathbb{Z}_{p}$, computes

$$
R_{\tau}=g_{1}^{-c_{\tau, 0}} \cdot\left(g_{1}^{r}\right)^{\rho_{\tau}}, \quad S_{\tau}=\left(\left(g_{1}^{\frac{z}{v}}\right)^{c_{\tau, 1}} \cdot\left(g_{1}^{\frac{r}{v}}\right)^{\rho_{\tau}} \cdot\left(g_{1}^{\frac{z}{v}}\right)^{\sum_{j=1}^{T} c_{j, 1}^{\prime} m_{j}}\right)^{\frac{1}{\xi_{k}}}
$$

and returns $\sigma=\left(Z_{k}, \sigma_{\Delta_{k}}, R_{\tau}, S_{\tau}\right)$ to $\mathcal{A}$.

Note that the signature is correctly distributed as in Game 7 , since $R_{\tau}$ is a uniformly distributed $\mathbb{G}_{1}$ element, and

$$
\begin{aligned}
S_{\tau} & =\left(\left(g_{1}^{\frac{z}{v}}\right)^{c_{\tau, 1}} \cdot\left(g_{1}^{\frac{r}{v}}\right)^{\rho_{\tau}} \cdot\left(g_{1}^{\frac{z}{v}}\right)^{\sum_{j=1}^{T} c_{j, 1}^{\prime} m_{j}}\right)^{\frac{1}{\xi_{k}}} \\
& =\left(\left(g_{1}^{z}\right)^{c_{\tau, 1}} \cdot\left(g_{1}^{r}\right)^{\rho_{\tau}} \cdot\left(g_{1}^{z}\right)^{\sum_{j=1}^{T} c_{j, 1}^{\prime} m_{j}}\right)^{\frac{1}{v \xi_{k}}} \\
& =\left(g_{1}^{c_{\tau, 0}} \cdot\left(g_{1}^{z}\right)^{c_{\tau, 1}} \cdot g_{1}^{-c_{\tau, 0}} \cdot\left(g_{1}^{r}\right)^{\rho_{\tau}} \cdot g_{1}^{\sum_{j=1}^{T}\left(c_{j, 1}^{\prime} z\right) m_{j}}\right)^{\frac{1}{z_{k}}} \\
& =\left(\mathrm{H}(\tau) \cdot R_{\tau} \cdot g_{1}^{\sum_{j=1}^{T} c_{j}^{\prime}(z) m_{j}}\right)^{\frac{1}{z_{k}}}=\left(\mathrm{H}(\tau) \cdot R_{\tau} \cdot \prod_{j=1}^{T} g_{1}^{c_{j}^{\prime}(z) m_{j}}\right)^{\frac{1}{z_{k}}} \\
& =\left(\mathrm{H}(\tau) \cdot R_{\tau} \cdot \prod_{j=1}^{T} \mathrm{H}^{\prime}(j)^{m_{j}}\right)^{\frac{1}{z_{k}}}
\end{aligned}
$$

- If $k=\mu$ : $\mathcal{B}$ runs $\vec{c}_{\tau} \leftarrow \mathrm{H}$.TrapEval $(\mathrm{td}, \tau)$, and $\vec{c}_{j}^{\prime} \leftarrow \mathrm{H}^{\prime}$.TrapEval $\left(\mathrm{td}^{\prime}, j\right)$ for all $j=1$ to $T$. It sets $R_{\tau}=g_{1}^{-c_{\tau, 0}}$ and computes

$$
S_{\tau}=\left(g_{1}^{c_{\tau, 1}} \cdot g_{1}^{\sum_{j=1}^{T} c_{j, 1}^{\prime} m_{j}}\right)^{\frac{1}{\xi_{\mu}}}
$$

and returns $\sigma=\left(Z_{\mu}, \sigma_{\Delta_{\mu}}, R_{\tau}, S_{\tau}\right)$ to $\mathcal{A}$. 
Chapter 3. Improving Efficiency: Linearly Homomorphic Signatures with Sublinear Public Keys via APHFs

As one can check, such signature is distributed as a signature in Game 7: $R_{\tau}=g_{1}^{-c_{\tau, 0}}$ as in the definition of Game 7 (for the $\mu$-th dataset) while for $S_{\tau}$ we have

$$
\begin{aligned}
S_{\tau} & =\left(g_{1}^{c_{\tau, 1}} \cdot g_{1}^{\sum_{j=1}^{T} c_{j, 1}^{\prime} m_{j}}\right)^{\frac{1}{\xi_{\mu}}}=\left(g_{1}^{z c_{\tau, 1}} \cdot g_{1}^{z \sum_{j=1}^{T} c_{j, 1}^{\prime} m_{j}}\right)^{\frac{1}{z \xi_{\mu}}} \\
& =\left(g_{1}^{c_{\tau}, 0} \cdot g_{1}^{z c_{\tau, 1}} \cdot g_{1}^{-c_{\tau, 0}} \cdot g_{1}^{\sum_{j=1}^{T}\left(c_{j, 1}^{\prime} z\right) m_{j}}\right)^{\frac{1}{z_{\mu}}} \\
& =\left(\mathrm{H}(\tau) \cdot R_{\tau} \cdot g_{1}^{\sum_{j=1}^{T} c_{j}^{\prime}(z) m_{j}}\right)^{\frac{1}{z_{\mu}}}=\left(\mathrm{H}(\tau) \cdot R_{\tau} \cdot \prod_{j=1}^{T} g_{1}^{c_{j}^{\prime}(z) m_{j}}\right)^{\frac{1}{z_{\mu}}} \\
& =\left(\mathrm{H}(\tau) \cdot R_{\tau} \cdot \prod_{j=1}^{T} \mathrm{H}^{\prime}(j)^{m_{j}}\right)^{\frac{1}{z_{\mu}}}
\end{aligned}
$$

Forgery: Let $\left(\mathcal{P}_{\Delta^{*}}^{*}, \sigma^{*}, \vec{m}^{*}\right)$ be the forgery returned by the adversary. $\mathcal{B}$ proceeds exactly as the challenger in Game 7 in order to compute $\hat{R}, \hat{S}, \overrightarrow{\hat{m}}$.

By definition, if Game 7 outputs 1 , since $\left(\mathcal{P}_{\Delta^{*}}^{*}, \sigma^{*}, \vec{m}^{*}\right)$ verifies correctly, the following two equations hold

$$
\begin{gathered}
e\left(S^{*}, Z_{\mu}\right)=\Lambda \cdot e\left(R^{*}, g_{2}\right) \cdot \prod_{j=1}^{T} \text { H.PubEval }\left(\text { pek }^{\prime}, j\right)^{m_{j}^{*}}, \\
\left.e\left(\hat{S}, Z_{\mu}\right)=\Lambda \cdot e\left(\hat{R}, g_{2}\right) \cdot \prod_{j=1}^{T} \text { H.PubEval' } \text { pek }^{\prime}, j\right)^{\hat{m}_{j}}
\end{gathered}
$$

where $\Lambda=\prod_{\tau \in \mathcal{L}^{*}}$ H.PubEval(pek, $\left.\tau\right)^{f_{\tau}^{*}}$. If we divide the two equations and consider that by definition of Game 7 , it must be $S^{*} \neq \hat{S}$ and $R^{*} \neq \hat{R}$, then we obtain:

$$
\begin{aligned}
\frac{S^{*}}{\hat{S}} & =\left(\frac{R^{*}}{\hat{R}} \cdot \prod_{j=1}^{T} \mathrm{H}^{\prime}(j)^{m_{j}^{*}-\hat{m}_{j}}\right)^{\frac{1}{z \xi_{\mu}}}=\left(\frac{R^{*}}{\hat{R}} \cdot \prod_{j=1}^{T} g_{1}^{c_{j, 1}^{\prime} z\left(m_{j}^{*}-\hat{m}_{j}\right)}\right)^{\frac{1}{z \xi_{\mu}}} \\
& =\left(\frac{R^{*}}{\hat{R}}\right)^{\frac{1}{z \xi_{\mu}}} \cdot\left(\prod_{j=1}^{T} g_{1}^{c_{j, 1}^{\prime}\left(m_{j}^{*}-\hat{m}_{j}\right)}\right)^{\frac{1}{\xi_{\mu}}}
\end{aligned}
$$

Therefore $\mathcal{B}$ can compute

$$
W=\frac{R^{*}}{\hat{R}}, \quad W^{\prime}=\left(\frac{S^{*}}{\hat{S}}\right)^{\xi_{\mu}} \cdot g_{1}^{\sum_{j=1}^{T} c_{j, 1}^{\prime}\left(\hat{m}_{j}-m_{j}^{*}\right)}
$$


Chapter 3. Improving Efficiency: Linearly Homomorphic Signatures with Sublinear Public Keys via APHFs

and returns $\left(W, W^{\prime}\right)$ as a solution for the FDHI assumption.

To see that $\left(W, W^{\prime}\right)$ is a solution for the FDHI assumption, i.e., $W^{\prime}=W^{1 / z}$, observe that by equation (3.2) it holds

$$
\begin{aligned}
\left(W^{\prime}\right)^{z} & =\left(\frac{S^{*}}{\hat{S}}\right)^{z \xi_{\mu}} \cdot g_{1}^{\sum_{j=1}^{T} z c_{j, 1}^{\prime}\left(\hat{m}_{j}-m_{j}^{*}\right)} \\
& =\left[\left(\frac{R^{*}}{\hat{R}}\right)^{\frac{1}{z \xi_{\mu}}} \cdot\left(\prod_{j=1}^{T} g_{1}^{c_{j, 1}^{\prime}\left(m_{j}^{*}-\hat{m}_{j}\right)}\right)^{\frac{1}{\xi_{\mu}}}\right]^{z \xi_{\mu}} \cdot g_{1}^{\sum_{j=1}^{T} z c_{j, 1}^{\prime}\left(\hat{m}_{j}-m_{j}^{*}\right)} \\
& =\frac{R^{*}}{\hat{R}}=W
\end{aligned}
$$

Note that the simulation of Game 7 provided by $\mathcal{B}$ to $\mathcal{A}$ is statistically close (by a factor $\gamma+\gamma^{\prime}$ due to the use of TrapGen in $\mathrm{H}$ and $\mathrm{H}^{\prime}$ ) to the real execution of Game 7. Then, it is easy to see that if Game 7 outputs $1, \mathcal{B}$ is able to compute the solution of the FDHI problem, as described above. In conclusion, if $\operatorname{Pr}\left[G_{7}(\mathcal{A})\right] \geq \epsilon$ then $\mathcal{B}$ has advantage at least $\left(\delta^{\prime} \epsilon\right)-\gamma-\gamma^{\prime}$ in solving FDHI.

Finally, we note that when instantiated with our APHF $\mathrm{H}_{\text {sqrt }}$ from Section 3.3.3. the programmability properties stated in the theorem hold with probability $\delta^{\prime}=1$ while the property of statistically-close trapdoor keys holds in a perfect sense, i.e., $\gamma=0$ and $\gamma^{\prime}=0$.

\subsubsection{Context-Hiding Security}

In this section, we prove the context-hiding security of our linearly homomorphic signature scheme.

Theorem 12. First notice that since in our case there is no hiding procedure (i.e., one can see Hide as the identity function and HVerify as Ver), correctness and unforgeability follow trivially.

In order to prove context-hiding security, we construct below a simulator and then show that its signatures are perfectly indistinguishable from the ones obtained through a run of the Eval algorithm.

Simulator $\operatorname{Sim}\left(\mathrm{sk}, \mathcal{P}_{\Delta}, \overrightarrow{\tilde{m}}\right)$. Parse the simulator's input as sk $=\left(\mathrm{sk}^{\prime}, K, \hat{K}\right.$, sek, sek' $)$, $\mathcal{P}_{\Delta}=\left(\left(f_{1}, \ldots, f_{\ell}\right), \tau_{1}, \ldots, \tau_{\ell}, \Delta\right)$ and $\tilde{\vec{m}}=\left(\tilde{m}_{1}, \ldots, \tilde{m}_{T}\right)$. With this information, the simulator computes the following values:

- $\left\{R_{i}=g_{1}^{F_{\hat{K}}\left(\Delta \mid \tau_{i}\right)}\right\}_{i=1}^{\ell}$ where $\left\{\tau_{i}\right\}_{i=1}^{\ell}$ are the labels in $\mathcal{P}_{\Delta}$.

- $\left\{\mathcal{H}_{\tau_{i}}=: \text { H.PriEval }\left(\text { sek, } \tau_{i}\right)\right\}_{i=1}^{\ell}$ and $\left\{\tilde{\mathcal{H}}_{j}=: \text { H.PriEval }{ }^{\prime}\left(\text { sek }^{\prime}, j\right)\right\}_{j=1}^{T}$. 
- $Z=g_{2}^{z}$, where $z \leftarrow F_{K}(\Delta)$.

- $\sigma_{\Delta} \leftarrow \operatorname{Sign}^{\prime}\left(\mathrm{sk}^{\prime}, \Delta \mid Z\right)$.

- $R=\prod_{i=1}^{\ell} R_{i}^{f_{i}}$

$-S=\left(\prod_{i=1}^{\ell} \mathcal{H}_{\tau_{i}}^{f_{i}} \cdot R \cdot \prod_{j=1}^{T} \tilde{\mathcal{H}}_{j}^{\tilde{m}_{j}}\right)^{1 / z}$.

The simulator finally outputs a signature $\sigma^{1}=\left(\sigma_{\Delta}, Z, R, S\right)$.

InDisTinguishabiLity OF SIGNATURES. Here we show that our simulator allows for context hiding security. Fix any choice of $($ sk, vk $) \in \operatorname{Key} \operatorname{Gen}\left(1^{\lambda}, \mathcal{L}\right)$, $\mathcal{P}_{\Delta}=\left(\left(f_{1}, \ldots, f_{\ell}\right), \tau_{1}, \ldots, \tau_{\ell}, \Delta\right)$, and $\vec{m}_{1}, \ldots, \vec{m}_{\ell} \in \mathcal{M}^{T}$. For all $i=1$ to $\ell$, we have $\sigma_{i}=\operatorname{Sign}\left(\right.$ sk, $\left.\Delta, \tau_{i}, \vec{m}_{i}\right)$ with $\sigma_{i}=\left(\sigma_{\Delta, i}, Z_{i}, R_{i}, S_{i}\right)$, and notice that since the scheme $\Sigma^{\prime}$ is deterministic fixing $\mathrm{sk}^{\prime}, \Delta, \tau_{1}, \vec{m}_{1}, \ldots, \tau_{\ell}, \vec{m}_{\ell}$ fixes also all the signatures $\sigma_{1}, \ldots, \sigma_{\ell}$.

Let $\sigma^{0} \leftarrow \operatorname{Eval}\left(\mathrm{vk}, \sigma_{1}, \ldots, \sigma_{\ell}\right)$ and recall that by construction the signature $\sigma^{0}=$ $\left(\sigma_{\Delta}^{\prime}, Z^{\prime}, R^{\prime}, S^{\prime}\right)$ consists of:

- $Z^{\prime}=Z_{1}=g_{2}^{z}$, where $z \leftarrow F_{K}(\Delta)$.

- $\sigma_{\Delta}^{\prime}=\sigma_{\Delta, 1} \leftarrow \operatorname{Sign}^{\prime}\left(\mathrm{sk}^{\prime}, \Delta \mid Z\right)$.

- $R^{\prime}=\prod_{i=1}^{\ell} R_{i}^{f_{i}}$, where $R=g_{1}^{r_{i}}$ and $r_{i} \leftarrow F_{\hat{K}}\left(\Delta \mid \tau_{i}\right)$.

- $S^{\prime}=\prod_{i=1}^{\ell} S_{i}^{f_{i}}$.

Let us now assume that we have a distinguisher $\mathcal{D}$ which is given $I=(\mathrm{sk}$, vk, $\left.\mathcal{P}_{\Delta}, \vec{m}_{1}, \sigma_{1}, \ldots, \vec{m}_{\ell}, \sigma_{\ell}\right)$ and either one of $\sigma^{0} \leftarrow \operatorname{Eval}\left(\mathrm{vk}, f,\left\{\sigma_{i}\right\}_{i=1}^{\ell}\right)$ or $\sigma^{1} \leftarrow$ $\operatorname{Sim}\left(s k, \mathcal{P}_{\Delta}, \overrightarrow{\tilde{m}}\right)$.

In what follows we show that $\sigma^{0}$ and $\sigma^{1}$ are distributed identically, that is any $\mathcal{D}$ has advantage 0 in distinguishing the two cases. To prove this, we are going to compare the two signatures $\sigma^{0}, \sigma^{1}$ element by element.

$$
\sigma_{\Delta}^{\prime}=\sigma_{\Delta, 1} \quad \mid \quad \sigma_{\Delta} \leftarrow \operatorname{Sign}^{\prime}\left(\mathrm{sk}^{\prime}, \Delta \mid Z\right)
$$

Since the scheme $\Sigma^{\prime}$ is deterministic and by construction of $\sigma_{\Delta, 1}$ (see above), one can see that $\sigma_{\Delta}$ and $\sigma_{\Delta}^{\prime}$ are equal. 
Chapter 3. Improving Efficiency: Linearly Homomorphic Signatures with Sublinear Public Keys via APHFs

$$
Z^{\prime}=Z_{1}=g_{2}^{z} \text {, where } z \leftarrow F_{K}(\Delta) \quad \mid \quad Z=g_{2}^{z} \text {, where } z \leftarrow F_{K}(\Delta)
$$

The two elements are clearly equal because of the PRF ( $z$ is uniquely determined given $K, \Delta)$.

$$
R^{\prime}=\prod_{i=1}^{\ell} R_{i}^{f_{i}} \quad \mid \quad R=\prod_{i=1}^{\ell} R_{i}^{f_{i}}
$$

By the construction of the simulator the elements $\left\{R_{i}\right\}_{i=1}^{\ell}$ are the same on both sides since in both cases are generated deterministically using the PRF $F$ with seed $\hat{K}$. This implies $R=R^{\prime}$.

$$
S^{\prime}=\prod_{i=1}^{\ell} S_{i}^{f_{i}}
$$

$$
S=\left(\prod_{i=1}^{\ell} \mathcal{H}_{\tau_{i}}^{f_{i}} \cdot R \cdot \prod_{j=1}^{T} \tilde{\mathcal{H}}_{j}^{\tilde{m}_{j}}\right)^{1 / z}
$$

We claim that $S^{\prime}=S$. To see this, let us recall how each $S_{i}$ is defined: by the signing algorithm we have $S_{i}=\left(\mathcal{H}_{\tau_{i}} \cdot R_{i} \cdot \prod_{j=1}^{T} \tilde{\mathcal{H}}_{j}^{m_{i, j}}\right)^{1 / z}$. Therefore

$$
S^{\prime}=\prod_{i=1}^{\ell}\left(\mathcal{H}_{\tau_{i}}^{f_{i}} \cdot R_{i}^{f_{i}} \cdot \prod_{j=1}^{T} \tilde{\mathcal{H}}_{j}^{f_{i} m_{i, j}}\right)^{1 / z}=\left(\prod_{i=1}^{\ell} \mathcal{H}_{\tau_{i}}^{f_{i}} \cdot R \cdot \prod_{j=1}^{T} \tilde{\mathcal{H}}_{j}^{\sum_{i=1}^{\ell} f_{i} m_{i, j}}\right)^{1 / z}=S
$$

where the last equality follows from the fact that $\tilde{\vec{m}}=: \sum_{i=0}^{\ell} f_{i} \vec{m}_{i}$.

\subsection{Short Signatures with Shorter Public Keys from Bilinear Maps}

In this section we describe how to use APHFs to construct in a generic fashion standard-model signature schemes over bilinear groups. We propose two constructions that are provably-secure under the $q$-Strong Diffie-Hellman [28] and the $q$-Diffie-Hellman [27] assumptions. These constructions are the analogues of the schemes in [83] and [82] respectively. The basic idea behind the constructions is to replace a standard $(m, 1)$-PHF with an $(m, 1, d)$-APHF. In fact, in this context, having a secretly-computable $\mathrm{H}$ does not raise any issue when using $\mathrm{H}$ in the signing procedure as the signer already uses a secret key. At the same time, for verification purposes, computing the (public) isomorphic copy of $\mathbf{H}$ in the target group is also sufficient. Our proof confirms that the $(m, 1, d)$-programmability can still be used to control the size of the randomness in the same way as in [83, 82]. One difference in the security proof is that the schemes in [83, 82] are based on the $q$ - $(\mathrm{S}) \mathrm{DH}$ assumption, where $q$ is the number of signing queries made by the 
Chapter 3. Improving Efficiency: Linearly Homomorphic Signatures with Sublinear Public Keys via APHFs

adversary, whereas ours have to rely on the $(q+d-1)-(\mathrm{S}) \mathrm{DH}$ problem. Since our instantiations use $d=2$, the difference (when considering concrete security) is very minor.

When plugging into these generic constructions our new APHF, $\mathrm{H}_{\mathrm{acfs}}$, described in Section 3.3.2, which is $(m, 1,2)$-programmable, we obtain schemes that, for signing $\ell$-bits messages, allow for public keys of length $O(m \sqrt{\ell})$ as in [116].

We describe the scheme based on $q$-SDH in Section 3.5.1, and the one based on $q-\mathrm{DH}$ in Section 3.5.2. As discussed in [82, the advantage of the scheme from $q$-DH compared to the one from $q-\mathrm{SDH}$ is to be based on a weaker assumption.

\subsubsection{A q-Strong Diffie-Hellman Based Solution}

In this section we revisit the $q$-SDH based solution of [83]. The signature $\Sigma_{\mathrm{qSDH}}=$ (KeyGen, Sign, Ver) is as follows:

$\operatorname{KeyGen}\left(1^{\lambda}\right)$. Let $\lambda$ be the security parameter, and let $\ell=\ell(\lambda)$ and $\rho=\rho(\lambda)$ be arbitrary polynomials. Our scheme can sign messages in $\{0,1\}^{\ell}$ using randomness in $\{0,1\}^{\rho}$. The key generation algorithm works as follows:

- Run bgp $\stackrel{\$}{\leftarrow} \mathcal{G}\left(1^{\lambda}\right)$ to generate the bilinear groups parameters bgp = $\left(p, \mathbb{G}_{1}, \mathbb{G}_{2}, \mathbb{G}_{T}, g_{1}, g_{2}, e\right)$ where $\mathbb{G}_{1}, \mathbb{G}_{2}$ and $\mathbb{G}_{T}$ are asymmetric groups of prime order $p \approx 2^{\lambda}, g_{1} \in \mathbb{G}_{1}, g_{2} \in \mathbb{G}_{2}$ are generators and $e: \mathbb{G}_{1} \times \mathbb{G}_{2} \rightarrow$ $\mathbb{G}_{T}$ is an efficiently computable, non-degenerate bilinear map.

- Run (sek, pek) $\stackrel{\$}{\leftarrow}$ H.Gen $\left(1^{\lambda}\right.$, bgp $)$ to generate the keys of the asymmetric hash function.

- Choose a random $x \stackrel{\$}{\leftarrow} \mathbb{Z}_{p}^{*}$ and set $X \leftarrow g_{2}^{x}$. Return vk = (bgp, pek, $\left.X\right)$ and $\mathrm{sk}=(\operatorname{sek}, x)$.

Sign(sk, $M)$. The signing algorithm takes as input the secret key sk, and a message $M \in\{0,1\}^{\ell}$. It starts by generating a random $r \stackrel{\$}{\leftarrow}\{0,1\}^{\rho}$. Next, it computes $\sigma=\mathrm{H}$.PriEval (sek, $M)^{\frac{1}{x+r}}$ and outputs $(\sigma, r)$.

$\operatorname{Ver}(\mathrm{vk}, M,(\sigma, r))$. To check that $(\sigma, r)$ is a valid signature, check that $r$ is of length $\rho$ and that

$$
e\left(\sigma, X \cdot g_{2}^{r}\right)=\text { H.PubEval }(\text { pek, } M)
$$

We state the security of the scheme in the following theorem. We note that for simplicity our proof assumes an $(m, 1, d)$-APHF for $d=2$, which matches our realization. A generalization of the theorem for a generic $d$ can be immediately obtained, in which case one would rely on the $(q+d-1)$-SDH assumption.

Theorem 15. Assume that $\mathcal{G}$ is a bilinear group generator such that the $(q+1)$ SDH assumption holds in $\mathbb{G}_{1}$ and $\mathrm{H}$ is $(m, 1,2, \gamma, \delta)$-programmable, then $\Sigma_{\mathbf{q S D H}}$ 
Chapter 3. Improving Efficiency: Linearly Homomorphic Signatures with Sublinear Public Keys via APHFs

is a secure signature scheme. More precisely, let $\mathcal{B}$ be an efficient (probabilistic) algorithm that runs in time $t$, asks (up to) $q$ signing queries and produces a valid forgery with probability $\epsilon$, then there exists an equally efficient algorithm $\mathcal{A}$ that confutes the $(q+1)-S D H$ assumption with probability

$$
\epsilon^{\prime} \geq \frac{\delta}{q}\left(\epsilon-\gamma-\frac{q}{p}-\frac{q^{m+1}}{2^{\rho m}}\right)
$$

Proof. This proof is almost identical to the corresponding one from [83], we rewrite it here mainly to show how to use APHFs in place of standard PHFs.

Let $\mathcal{B}$ be an adversary against the signature scheme. Assuming that $\mathcal{B}$ asks (up to) $q$ signing queries, and denote with $M_{i}$ the $i$-th queried message and with $\left(\sigma_{i}, r_{i}\right)$ the corresponding signature. Also, let $M^{*},\left(\sigma^{*}, r^{*}\right)$ be the produced forgery. We distinguish two types of forgeries:

Type I forgery: It holds that $r^{*}=r_{i}$ for some $i \in[q]$.

Type II forgery: It holds that $r^{*} \neq r_{i} \forall i \in[q]$.

Notice that these two cases are mutually exclusive and completely cover the set of possible forgeries. Now we show that both types of forgeries can be used to violate the $(q+1)-\mathrm{SDH}$ assumption.

Lemma 16 (Type I forgeries). Let $\mathcal{B}$ be a type I forger that breaks the signature scheme with advantage $\epsilon_{1}$ (and making up to q signature queries). Then there exists an (equally efficient) adversary $\mathcal{A}$ that breaks the $(q+1)-S D H$ assumption with advantage $\epsilon^{\prime}$, where

$$
\epsilon^{\prime} \geq \frac{\delta}{q}\left(\epsilon_{1}-\gamma-\frac{q}{p}-\frac{q^{m+1}}{2^{\rho m}}\right)
$$

We prove the lemma via a sequence of games. We denote with $G_{i}$ the event that Game $i$ outputs 1, i.e., that $\mathcal{B}$ (successfully) forges in Game $i$.

Game 0 This game is the standard existential unforgeability experiment $\operatorname{Exp}_{\mathcal{B}, \Sigma}^{\text {UF-CMA }}$. Clearly,

$$
\operatorname{Pr}\left[G_{0}\right]=\epsilon_{1}
$$

Game 1 This is the same as the previous game but the parameter of the APHF are generated using $\mathrm{H}$.TrapGen (rather than H.Gen). More precisely, $\mathcal{A}$ runs H.TrapGen $\left(1^{\lambda}, g_{1}, h_{1}, g_{2}, h_{2}\right)$, where $g_{1}, g_{2}$ are, randomly chosen, generators and $h_{1}=g_{1}^{\alpha}, h_{2}=g_{2}^{\alpha}$ for a randomly chosen $\alpha \stackrel{\$}{\leftarrow} \mathbb{Z}_{p}$. By the $\gamma$-closeness of the trapdoor keys, we have:

$$
\operatorname{Pr}\left[G_{1}\right] \geq \operatorname{Pr}\left[G_{0}\right]-\gamma
$$


Chapter 3. Improving Efficiency: Linearly Homomorphic Signatures with Sublinear Public Keys via APHFs

Game 2 In this game we do the following changes. First, we choose the $r_{i}$ 's used to answer signing queries all in advance (rather than one by one when needed). Since the $r_{i}$ 's are chosen at random and independently anyway this change cannot affect $\mathcal{B}$ 's advantage at all. Second, we modify the way $g_{1}, h_{1}, g_{2}, h_{2}$ are chosen when executing H.TrapGen. Specifically, let $\hat{g}_{1}$ be a generator of $\mathbb{G}_{1}$ and $\hat{g}_{2}$ be a generator of $\mathbb{G}_{2}$. We choose $i^{*} \in_{R}\{1, \ldots, q\}$ and we set $r^{*}=r_{i^{*}}, R=\cup_{i=1}^{q} r_{i}, R^{*}=R \backslash\left\{r^{*}\right\}$ and $R^{*, i}=R \backslash\left\{r^{*}, r_{i}\right\}$. Next, we define the polynomials $p^{*}(z)=\prod_{r \in R^{*}}(z+r) \bmod p$ and $p(z)=$ $p^{*}(z)\left(z+r^{*}\right) \bmod p$. Notice that both polynomials are of degree $\leq q$. Thus, from $\hat{g}_{1}, \hat{g}_{1}^{x}, \ldots, \hat{g}_{1}^{x^{q}}$ it is possible to compute $g_{1}=\hat{g}_{1}^{p^{*}(x)}$ and $h_{1}=\hat{g}_{1}^{p(x)}$. Next we set $g_{2}=\hat{g}_{2}, X=g_{2}^{x}$ and $h_{2}=g_{2}^{\left(x+r^{*}\right)}$. The distribution of $g_{1}, g_{2}$ is identical to the one in Game 1. The only difference might occur in the case $p(x)=0$, as in this case $g_{1}, h_{1}$ would not be generators. By the SchwartzZippel lemma [106, 118], however, this happens only with probability at most $q / p$. Thus

$$
\operatorname{Pr}\left[G_{2}\right] \geq \operatorname{Pr}\left[G_{1}\right]-\frac{q}{p}
$$

Game 3 Let $\operatorname{Bad}_{3}$ be the event that the same $r_{i}$ is used to sign more than $m$ different messages. This means that if $\mathrm{Bad}_{3}$ occurs there are, at least, $m+1$ indices $i_{1}, \ldots, i_{m+1}$ such that $r_{i_{1}}=\ldots=r_{i_{m+1}}$. On $q$ signing queries there might be up to $\left(\begin{array}{c}q \\ m+1\end{array}\right) \leq q^{m+1}$ such tuples. Moreover, a given tuple is of the form $r_{i_{1}}=\ldots=r_{i_{m+1}}$ with probability $2^{\rho} / 2^{\rho(m+1)}$. This means that

$$
\operatorname{Pr}\left[\operatorname{Bad}_{3}\right] \leq \frac{q^{m+1}}{2^{\rho m}}
$$

We modify Game 2, by letting that the simulation aborts if $\operatorname{Bad}_{3}$ occurs. Thus,

$$
\operatorname{Pr}\left[G_{3}\right] \geq \operatorname{Pr}\left[G_{2}\right]-\frac{q^{m+1}}{2^{\rho m}}
$$

Game 4 Let $\operatorname{Bad}_{4}$ be the event that $\mathcal{B}$ outputs a value $r^{*}$ such that $r^{*}=r_{i}$, but $i \neq i^{*}$. We modify the previous game by imposing that the simulation aborts if $\mathrm{Bad}_{4}$ occurs. Thus,

$$
\operatorname{Pr}\left[G_{4}\right]=\operatorname{Pr}\left[G_{4} \wedge \neg \operatorname{Bad}_{4}\right]=\frac{1}{q} \operatorname{Pr}\left[G_{3}\right]
$$

Game 5 Let $\operatorname{Bad}_{5}$ be the event that either there is an index $i \in[q]$ such that $r_{i}=r^{*}$ such that $c_{M_{i}, 0} \neq 0$, or it occurs $c_{M^{*}, 0}=0$. Game 5 proceeds as Game 4 except that it aborts if $\operatorname{Bad}_{5}$ occurs. Using the programmability of $\mathrm{H}$, we can bound the probability of $\operatorname{Bad}_{5}$. Precisely, we have that $\operatorname{Pr}\left[\neg \operatorname{Bad}_{5}\right] \geq \delta$, from which we have

$$
\operatorname{Pr}\left[G_{5}\right]=\operatorname{Pr}\left[G_{5} \wedge \neg \mathrm{Bad}_{5}\right]=\delta \operatorname{Pr}\left[G_{4}\right]
$$


Chapter 3. Improving Efficiency: Linearly Homomorphic Signatures with Sublinear Public Keys via APHFs

Game 6 We further modify the simulation by using the alternative signing mechanism, from [28]. In particular, to sign the $i$-th queried message $M_{i}$, one proceeds as follows. First, compute $\vec{c}_{M_{i}} \leftarrow \mathrm{H}$. TrapEval(td, $\left.M_{i}\right)$. Notice that by the setting of $h_{1}, h_{2}$ and by the definition of TrapEval, $\vec{c}_{M_{i}}$ is a degree-2 polynomial $c_{M_{i}}\left(x+r^{*}\right)$. Let us write $c_{M_{i}}\left(x+r^{*}\right)=c_{M_{i}, 0}+c_{M_{i}}^{\prime}\left(x+r^{*}\right)$, where $c_{M_{i}}^{\prime}\left(x+r^{*}\right)$ is the degree-2 polynomial obtained by simply removing the degree-0 term from $c_{M_{i}}\left(x+r^{*}\right)$. Hence, one computes

$$
\begin{aligned}
\sigma_{i} & =\text { H.PriEval }\left(\text { sek, } M_{i}\right)^{\frac{1}{x+r_{i}}}=\left(g_{1}^{c_{M_{i}, 0}+c_{M_{i}}^{\prime}\left(x+r^{*}\right)}\right)^{\frac{1}{x+r_{i}}} \\
& =\left(\hat{g}_{1}^{p^{*}(x) c_{M_{i}, 0}} \hat{g}_{1}^{p^{*}(x) c_{M_{i}}^{\prime}\left(x+r^{*}\right)}\right)^{\frac{1}{x+r_{i}}} \\
& =\hat{g}_{1}^{c_{M_{i}, 0} \prod_{r \in R^{*}, i}(x+r)} \hat{g}_{1}^{c_{M_{i}}^{\prime}\left(x+r^{*}\right) \prod_{r \in R^{*}, i}(x+r)}
\end{aligned}
$$

Moreover, for all the signing queries that do not cause $\mathrm{Bad}_{5}$, notice that $c_{M_{i}, 0}=0$ and thus such signing queries can be answered without any explicit knowledge of $x$. As a consequence,

$$
\operatorname{Pr}\left[G_{6}\right]=\operatorname{Pr}\left[G_{5}\right]
$$

Notice also that in Game 6, we are assuming that neither Bad $_{5}$ nor Bad $_{4}$ occur. This means that, for the the forged signature $\left(M^{*}, \sigma^{*}, r^{*}\right)$ one has that H.PriEval (sek, $\left.M^{*}\right)=g_{1}^{c_{M^{*}}\left(x+r^{*}\right)}$ and $c_{M^{*}, 0} \neq 0$. Using the same notation as above, using the $q$-SDH instance we can compute

$$
y=g_{1}^{\frac{c_{m_{i}}^{\prime}\left(x+r^{*}\right)}{x+r^{*}}}
$$

as $c_{m_{i}}^{\prime}\left(x+r^{*}\right)$ is a polynomial of degree $\leq 2$ without the constant term, i.e, $c_{m_{i}}^{\prime}\left(x+r^{*}\right)$ is divisible by $\left(x+r^{*}\right)$. We set

$$
\sigma^{\prime}=\left(\sigma^{*} \cdot y^{-1}\right)^{1 / c_{m^{*}, 0}}=g_{1}^{\frac{1}{x+r^{*}}}=\hat{g}_{1}^{\frac{p^{*}(x)}{x+r^{*}}}
$$

Using standard techniques [28, 83], $\sigma^{\prime}$ can be used to extract the required $\hat{g}_{1}^{\frac{1}{x+r^{*}}}$. This means that $\operatorname{Pr}\left[G_{6}\right] \leq \epsilon$. Finally, putting together the bounds from the games above yields the lemma.

Lemma 17 (Type II forgeries). Let $\mathcal{B}$ be a type II forger that breaks the signature scheme with advantage $\epsilon_{2}$ (and making up to q signature queries). Then there exist (equally efficient) adversaries $\mathcal{A}_{1}$, that breaks the $(q+1)$-SDH assumption with advantage $\epsilon$, and $\mathcal{A}_{2}$ that breaks the discrete logarithm problem with advantage $\epsilon_{\mathrm{DL}}$, where

$$
\epsilon+\epsilon_{\mathrm{DL}} \geq \epsilon_{2}-q / p-\gamma
$$


Chapter 3. Improving Efficiency: Linearly Homomorphic Signatures with Sublinear Public Keys via APHFs

Again we prove the lemma via a sequence of games, and use $G_{i}$ to denote the event that $\mathcal{B}$ (successfully) forges in Game $i$.

Game 0 This game is the standard existential unforgeability experiment $\operatorname{Exp}_{\mathcal{B}, \Sigma}^{\text {UF-CMA }}$. Clearly,

$$
\operatorname{Pr}\left[G_{0}\right]=\epsilon_{2}
$$

Game 1 This is the same as Game 1 above, (i.e. the parameter of the programmable hash function are generated using H.TrapGen (rather than H.Gen). Thus,

$$
\operatorname{Pr}\left[G_{1}\right] \geq \operatorname{Pr}\left[G_{0}\right]-\gamma
$$

Game 2 In this game we do the following changes. First, we choose the $r_{i}$ 's used to answer signing queries all in advance (rather than one by one when needed). Second, we modify the way $g_{1}, h_{1}, g_{2}, h_{2}$ are chosen when executing H.TrapGen. Specifically, let $\hat{g}_{1}$ be a generator of $\mathbb{G}_{1}$ and $\hat{g}_{2}$ be a generator of $\mathbb{G}_{2}$. We we set $R=\cup_{i=1}^{q} r_{i}$. Next we define the (degree- $q$ ) polynomial $p(z)=\prod_{r \in R}(z+r) \bmod p$. From $\hat{g}_{1}, \hat{g}_{1}^{x}, \ldots, \hat{g}_{1}^{x^{q}}$ it is possible to compute $g_{1}=\hat{g}_{1}^{p(x)}$ and $h_{1}=g_{1}=\hat{g}_{1}^{p(x)}$. Next we set $g_{2}=\hat{g}_{2}, X=g_{2}^{x}$, and $h_{2}=g_{2}^{\alpha}$ (for random $\alpha \stackrel{\$}{\leftarrow} \mathbb{Z}_{p}$ ). Note that the distribution of $g_{1}, g_{2}$ is identical with respect to Game 1. Again, the only difference might occur in the case when $p(x)=0$, as in this case $g_{1}$ would not be a generator. Thus

$$
\operatorname{Pr}\left[G_{2}\right] \geq \operatorname{Pr}\left[G_{1}\right]-\frac{q}{p}
$$

Game 3 Let $M^{*}$ be the message used in the forgery, and let $c_{M^{*}} \leftarrow \mathrm{H}$.TrapEval $(\mathrm{td}$, $\left.M^{*}\right)$. We define $\operatorname{Bad}_{3}$ as the event that $c_{M^{*}}(\alpha)=0$. Then, if $\operatorname{Bad}_{3}$ happens Game 3 aborts. It is not hard to show, that if $\operatorname{Bad}_{3}$ occurs, then one can break the discrete $\log$ assumption in the group $\mathbb{G}_{2}$ (which in turn is implied by the $(q+1)$-SDH assumption). Indeed, $c_{M^{*}}(\alpha)=c_{M^{*}, 0}+c_{M_{1}^{*}} \alpha$ is here a degree-1 polynomial in the variable $\alpha$, and the elements $g_{2}, g_{2}^{\alpha}$ are given as part of a challenge $\left(g_{1}, g_{1}^{\alpha}, \ldots, g_{1}^{\alpha^{q}}, g_{2}, g_{2}^{\alpha}\right)$ of our $(q+1)$-SDH assumption. The unknown discrete $\log \alpha$ can be then easily computed as $\alpha=-c_{M^{*}, 0} / c_{M^{*}, 1}$.

$$
\operatorname{Pr}\left[G_{3}\right] \geq \operatorname{Pr}\left[G_{2}\right]-\epsilon_{\mathrm{DL}}
$$

Game 4 We further modify the simulation by using the alternative signing mechanism, from [28]. In particular, to sign the $i$-th message $M_{i}$ one obtains $c_{M_{i}} \leftarrow$ H.TrapEval $\left(\mathrm{td}, M_{i}\right)$ and then computes

$$
\begin{aligned}
\sigma_{i} & =\text { H.PriEval }\left(\text { sek, } M_{i}\right)^{\frac{1}{x+r_{i}}}=\left(g_{1}^{c_{M_{i}}(\alpha)}\right)^{\frac{1}{x+r_{i}}}=\left(\hat{g}_{1}^{p(x) c_{M_{i}}(\alpha)}\right)^{\frac{1}{x+r_{i}}} \\
& =\hat{g}^{c_{M_{i}}(\alpha) \prod_{r \in R \backslash\left\{r_{i}\right\}}(x+r)}
\end{aligned}
$$


Chapter 3. Improving Efficiency: Linearly Homomorphic Signatures with Sublinear Public Keys via APHFs

Since all the signing queries, can be answered without any explicit knowledge of $x$ we have that

$$
\operatorname{Pr}\left[G_{4}\right]=\operatorname{Pr}\left[G_{3}\right]
$$

Notice also that since we are assuming that $\mathrm{Bad}_{3}$ does not occur we have that, from the produced forgery on $M^{*}$ we can extract

$$
\sigma^{\prime}=\left(\sigma^{*}\right)^{1 / c_{M^{*}}(\alpha)}=g_{1}^{\frac{1}{x+r^{*}}}=\hat{g}_{1}^{\frac{p(x)}{x+r^{*}}}
$$

Again, by using standard techniques [28, 83], $\sigma^{\prime}$ can be used to extract the required $\hat{g}_{1}^{\frac{1}{x+r^{*}}}$. Hence, $\operatorname{Pr}\left[G_{4}\right] \leq \epsilon$.

Finally, putting together the bounds from the games above yields the lemma.

\subsubsection{A $q$-Diffie Hellman based solution}

In this section we show how to revisit the $q$-DH based scheme of [82] in order to work with APHFs. Our construction uses a standard PHF as an additional building block. We construct a signature $\Sigma_{\mathrm{qDH}}=($ KeyGen, Sign, Ver) as follows:

KeyGen $\left(1^{\lambda}\right)$. Let $\lambda$ be the security parameter, and let $\ell=\ell(\lambda)$ and $\rho=\rho(\lambda)$ be arbitrary polynomials. The scheme can sign messages in $\{0,1\}^{\ell}$ using randomness in $\{0,1\}^{\rho}$. The key generation algorithm works as follows:

- Run bgp $\stackrel{\$}{\leftarrow} \mathcal{G}\left(1^{\lambda}\right)$ to generate the bilinear groups parameters bgp = $\left(p, \mathbb{G}_{1}, \mathbb{G}_{2}, \mathbb{G}_{T}, g_{1}, g_{2}, e\right)$ where $\mathbb{G}_{1}, \mathbb{G}_{2}$ and $\mathbb{G}_{T}$ are groups of prime order $p \approx 2^{\lambda}, g_{1} \in \mathbb{G}_{1}, g_{2} \in \mathbb{G}_{2}$ are generators and $e: \mathbb{G}_{1} \times \mathbb{G}_{2} \rightarrow \mathbb{G}_{T}$ is an efficiently computable, non-degenerate bilinear map.

- Run (sek, pek) $\stackrel{\$}{\leftarrow}$ H.Gen $\left(1^{\lambda}\right.$, bgp) to generate the keys of the asymmetric hash function.

- Let $\mathrm{D}=$ (PHF.Gen, PHF.Eval) be a group hash function [83] over $\mathbb{G}_{2}$ with input length $\rho$ such that $D$ is programmable using the algorithms (PHF.TrapGen, PHF.TrapEval). Run $(\kappa, \tau) \leftarrow$ PHF.TrapGen $\left(1^{\lambda}, g_{1}, g_{1}^{y}\right)$, for a random $y \stackrel{\$}{\leftarrow} \mathbb{Z}_{p}$.

- Return $\mathbf{v k}=($ bgp, pek, $\kappa)$ and sk $=($ sek, $\tau, y)$. In what follows, we use the same notation of [82], and use $\mathrm{d}(r)$ as a shorthand for $(a, b) \leftarrow$ $\operatorname{PHF}$. TrapEval $(\tau, r), \mathrm{d}(r)=a+y b$.

Sign(sk, $M)$. The signing algorithm takes as input the secret key sk, and a message $M \in\{0,1\}^{\ell}$. It starts by generating a random $r \in\{0,1\}^{\rho}$. Next, it computes $\sigma=$ H.PriEval (sek, $M)^{\frac{1}{\mathrm{~d}(r)}}$ and outputs $(\sigma, r)$. 
Chapter 3. Improving Efficiency: Linearly Homomorphic Signatures with Sublinear Public Keys via APHFs

$\operatorname{Ver}(\mathrm{vk}, M,(\sigma, r))$. To verify that $(\sigma, r)$ is a valid signature, check that $r$ is of length $\rho$, that $\mathrm{d}(r) \neq 0$ and that

$$
e(\sigma, \operatorname{PHF} . \operatorname{Eval}(r))=\text { H.PubEval }(\text { pek, } M)
$$

We prove the security of the scheme in the following theorem. We note that for simplicity our proof assumes an $(m, 1, d)$-APHF for $d=2$, which matches our realization. A generalization of the theorem for a generic $d$ can be immediately obtained, in which case one would rely on the $(q+d-1)-\mathrm{DH}$ assumption.

Theorem 16. Assume that $\mathcal{G}$ is a bilinear group generator such that the $(q+1)$ DH assumption holds in $\mathbb{G}_{1}, \mathrm{H}$ is an asymmetric $(m, 1,2, \gamma, \delta)$-programmable hash function, $\mathrm{D}$ is a $\left(1\right.$, poly, $\left.\gamma^{\prime}, \delta^{\prime}\right)$ programmable hash function over $\mathbb{G}_{2}$ then $\Sigma_{\mathrm{qDH}}$ is a secure signature scheme. More precisely let $\mathcal{B}$ be an efficient (probabilistic) algorithm that runs in time $t$, asks (up to) q signing queries and produces a valid forgery with probability $\epsilon_{1}$, then there exists an equally efficient algorithm $\mathcal{A}$ that confutes the $(q+1)$-DH assumption with probability

$$
\epsilon^{\prime} \geq \delta \delta^{\prime}\left(\frac{\epsilon_{1}}{q}-\gamma-\frac{q^{m}}{2^{\rho m}}\right)
$$

Proof. Again the proof is almost identical to the corresponding one from [83], we rewrite it here for completeness. Let $\mathcal{B}$ be an adversary against the signature scheme. Assuming that $\mathcal{B}$ asks (up to) $q$ signing queries we denote with $M_{i}$ the $i$-th queried message and with $\left(\sigma_{i}, r_{i}\right)$ the corresponding signature. Also, let $M^{*},\left(\sigma^{*}, r^{*}\right)$ be the produced forgery. We distinguish two types of forgeries

Type I forgery : It holds that $r^{*}=r_{i}$ for some $i \in[q]$.

Type II forgery : It holds that $r^{*} \neq r_{i} \forall i \in[q]$.

Notice that these two cases are mutually exclusive and completely cover the set of possible forgeries. Now we show that both types of forgeries can be used to violate the $(q+1)$-DH assumption.

Lemma 18 (Type I forgeries). Let $\mathcal{B}$ be a type I forgery that breaks the signature scheme with advantage $\epsilon_{1}$ (and making up to q signature queries). Then there exists an (equally efficient) adversary $\mathcal{A}$ that breaks the $(q+1)-D H$ assumption with advantage $\epsilon^{\prime}$, where

$$
\epsilon^{\prime} \geq \delta \delta^{\prime}\left(\frac{\epsilon_{1}}{q}-\gamma-\frac{q^{m}}{2^{\rho m}}\right)
$$

Again we prove the lemma via a sequence of games, and use $G_{i}$ to denote the event that $\mathcal{B}$ (successfully) forges in Game $i$. 
Chapter 3. Improving Efficiency: Linearly Homomorphic Signatures with Sublinear Public Keys via APHFs

Game 0 This game is the standard existential unforgeability experiment $\operatorname{Exp}_{\mathcal{B}, \Sigma}^{\text {UF-CMA }}$. Clearly,

$$
\operatorname{Pr}\left[G_{0}\right]=\epsilon_{1}
$$

Game 1 Let $\operatorname{Bad}_{1}$ be the event that the same $r_{i}$ is used more than $m$ times. We change the simulation by forcing an abort if $\operatorname{Bad}_{1}$ occurs. As done in lemma 16 we have that

$$
\operatorname{Pr}\left[G_{1}\right] \geq \operatorname{Pr}\left[G_{0}\right]-\frac{q^{m+1}}{2^{\rho m}}
$$

Game 2 In this game we do the following changes. First, we choose the $r_{i}$ 's used to answer signing queries all in advance (rather than one-by-one when needed). Since the $r_{i}$ 's are chosen randomly and independently anyway, this change cannot affect $\mathcal{B}$ 's advantage at all. Second, we guess the index $i$ such that $i=i^{*}$ and we abort if this does not happen (i.e. $\mathcal{B}$ outputs an $\left.r^{*} \neq r_{i}\right)$. Clearly,

$$
\operatorname{Pr}\left[G_{2}\right] \geq \frac{1}{q} \operatorname{Pr}\left[G_{1}\right]
$$

Game 3 In this game we do the following changes. First, the parameter of the asymmetric programmable hash function are generated using $\mathrm{H}$.TrapGen (rather than H.Gen). Next, we modify the way $g_{1}, h_{1}, g_{2}, h_{2}$ are chosen when executing $\mathrm{H}$.TrapGen. Specifically, let $\hat{g}_{1}$ be a generator of $\mathbb{G}_{1}$ and $\hat{g}_{2}$ be a generator of $\mathbb{G}_{2}$. Let $R=\cup_{i=1}^{q} r_{i}, R^{*}=R \backslash\left\{r^{*}\right\}$ and $R^{*, i}=R \backslash\left\{r^{*}, r_{i}\right\}$. We set

$$
g_{1}=\hat{g}_{1}^{\prod_{r \in R^{*}} \mathrm{~d}(r)} \quad h_{1}=\hat{g}_{1}^{\prod_{r \in R} \mathrm{~d}(r)} \quad g_{2}=\hat{g}_{2} \quad h_{2}=\hat{g}_{2}^{\mathrm{d}\left(r^{*}\right)}
$$

The $\gamma$-statistical closeness of H's trapdoor keys implies

$$
\operatorname{Pr}\left[G_{3}\right] \geq \operatorname{Pr}\left[G_{2}\right]-\gamma
$$

Game 4 Let $\mathrm{Bad}_{4}$ be the event that, letting $\vec{c}_{M_{i}} \leftarrow \mathrm{H}$. TrapEval(td, $\left.M_{i}\right)$, the following happens. Either $c_{M_{i}, 0} \neq 0$ for some $i$ for which $r_{i}=r^{*}$, or $c_{M^{*}, 0}=0$ (where $\vec{c}_{M^{*}} \leftarrow \mathrm{H}$.TrapEval (td, $\left.M^{*}\right)$ ). Game 4 proceeds as Game 3 except that it it aborts if $\mathrm{Bad}_{4}$ occurs. The programmability of $\mathrm{H}$ implies that

$$
\operatorname{Pr}\left[G_{4}\right]=\operatorname{Pr}\left[G_{3} \wedge \neg \mathrm{Bad}_{4}\right] \geq \delta \operatorname{Pr}\left[G_{3}\right]
$$

Game 5 Now we change the way signing queries are answered. Whenever a message $M_{i}$ is queried, the simulator computes $c_{M_{i}} \leftarrow \mathrm{H}$.TrapEval(td, $\left.m_{i}\right)$ and sets

$$
\sigma_{i}=\hat{g}_{1}^{c_{M_{i}}\left(\mathrm{~d}\left(r^{*}\right)\right) \prod_{r \in R^{*, i}} \mathrm{~d}(r)}
$$

Notice that it is possible to sign all the received signing queries as, by Game 4 , for all $r_{i}=r^{*}$, it holds $c_{M_{i}, 0}=0$. Game 5 is perfectly indistinguishable from Game 4, from $\mathcal{B}$ 's perspective, i.e.,

$$
\operatorname{Pr}\left[G_{5}\right]=\operatorname{Pr}\left[G_{4}\right]
$$


Chapter 3. Improving Efficiency: Linearly Homomorphic Signatures with Sublinear Public Keys via APHFs

Game 6 Now for each $r_{i}$ we compute $\left(a_{i}, b_{i}\right) \leftarrow \operatorname{PHF}$.TrapEval $\left(\tau, r_{i}\right)$ (for the received forgery we would get $\left.\left(a^{*}, b^{*}\right)\right)$. Let $\operatorname{Bad}_{6}$ be the event that, either $a_{i} \equiv 0 \bmod p$ for some $i$ such that $r^{*}=r_{i}$, or $a^{*} \neq 0$. If $\operatorname{Bad}_{6}$ occurs, Game 6 aborts. By the $\left(1\right.$, poly, $\left.\gamma^{\prime}, \delta^{\prime}\right)$ programmability of $\mathrm{D}$

$$
\operatorname{Pr}\left[G_{6}\right] \geq \delta^{\prime} \operatorname{Pr}\left[G_{5}\right]
$$

Now we embed the received $(q+1)$-DH challenge $\left(\hat{g}_{1}, \hat{g}_{1}^{y}, \ldots, \hat{g}_{1}^{y^{q+1}}, \hat{g}_{2}, \hat{g}_{2}^{y}\right)$ as input, and we proceed as before (but using the fact that we do not explicitly know $y$ ). It is easy to check that all signing queries can be answered. Moreover, once the forgery $\left(M^{*}, \sigma^{*}, r^{*}\right)$ is produced, we can extract a solution of the $(q+1)$-DH challenge as follows. First, since by Game $4 c_{M^{*}, 0} \neq 0$, we can write

$$
z=\left(\frac{\sigma^{*}}{\hat{g}_{1}^{c_{M}^{\prime}\left(\mathrm{d}\left(r^{*}\right)\right)} \prod_{r \in R^{*}}\left(a_{r}+y b_{r}\right)}\right)^{b^{*} / c_{M^{*}, 0}}
$$

where, $c_{M_{*}}^{\prime}\left(\mathrm{d}\left(r^{*}\right)\right)$ is the polynomial obtained from $c_{M^{*}}\left(\mathrm{~d}\left(r^{*}\right)\right)$ by removing the constant term $c_{M^{*}, 0}$ and dividing by $\left.\mathrm{d}\left(r^{*}\right)\right)$.

$$
\begin{aligned}
z & =\left(\frac{\text { H.PriEval }\left(\text { sek, } M^{*}\right)^{\frac{1}{\mathrm{~d}\left(r^{*}\right)}}}{\hat{g}_{1}^{c_{M^{*}}^{\prime}\left(\mathrm{d}\left(r^{*}\right)\right) \prod_{r \in R^{*}}\left(a_{r}+y b_{r}\right)}}\right)^{b^{*} / c_{M^{*}, 0}}=\left(\frac{g_{1}^{c_{M^{*}}\left(\mathrm{~d}\left(r^{*}\right)\right) / \mathrm{d}\left(r^{*}\right)}}{\hat{g}_{1}^{c_{M^{*}}\left(\mathrm{~d}\left(r^{*}\right)\right) \prod_{r \in R^{*}}\left(a_{r}+y b_{r}\right)}}\right)^{b^{*} / c_{m^{*}, 0}} \\
& =\left(\frac{g_{1}^{c_{M^{*}, 0} / \mathrm{d}\left(r^{*}\right)} \hat{g}_{1}^{c_{M}^{\prime}}\left(\mathrm{d}\left(r^{*}\right)\right) \prod_{r \in R^{*}}\left(a_{r}+y b_{r}\right)}{\hat{g}_{1}^{c_{M^{*}}^{\prime}\left(\mathrm{d}\left(r^{*}\right)\right) \prod_{r \in R^{*}}\left(a_{r}+y b_{r}\right)}}\right)^{b^{*} / c_{M^{*}, 0}}=\left(g_{1}^{c_{M^{*}, 0} / \mathrm{d}\left(r^{*}\right)}\right)^{b^{*} / c_{M^{*}, 0}} \\
& =\left(g_{1}^{c_{M^{*}, 0} /\left(y b^{*}\right)}\right)^{b^{*} / c_{M^{*}, 0}}=g_{1}^{1 / y}
\end{aligned}
$$

Finally, by using techniques from [28] one can easily get the desired result $\hat{g}_{1}^{1 / y}$.

Lemma 19 (Type II forgeries). Let $\mathcal{B}$ be a type II forger that breaks the signature scheme with advantage $\epsilon_{2}$ (and making up to q signature queries). Then there exists an (equally efficient) adversary $\mathcal{A}$ that breaks the $(q+1)-D H$ assumption with advantage $\epsilon^{\prime}$ and an adversary that breaks the discrete logarithm assumption with advantage $\epsilon^{\prime \prime}$ where

$$
\epsilon^{\prime}+\delta^{\prime} \epsilon^{\prime \prime} \geq \delta^{\prime}\left(\epsilon_{2}-\gamma\right)
$$

This lemma can be proved by easily adapting the proof of lemma 17 to this setting. 


\section{Extending Functionalities: Multi-Key Homomorphic Authenticators}

The object of this chapter is the study of Multi-Key Homomorphic Authenticators, both in terms of definitions and constructions.

\subsection{On the Concept of Multi-Key Homomor- phic Authenticators}

In this part of the thesis we will be using the generic word "authenticator", referring both to signatures and MACs. This approach is intentional: since the main difference between these two cryptographic tools basically relies on the nature of the verification procedure (public in the case on signatures and private when considering MACs), we think it is nice to have a unique generic framework in terms of definitions. Of course, we will differentiate between signatures and MACs when presenting the constructions. At a high level, Multi-Key Homomorphic Authenticators (multi-key HAs) are like Homomorphic Authenticators (HAs) with the additional property that one can execute a program $\mathcal{P}$ on data authenticated using different secret keys. In multi-key HAs, Bob verifies using the verification keys of all users that provided inputs to $\mathcal{P}$. These features make multi-key HAs a perfect candidate for applications where multiple users gather and outsource data. For example, consider a network of sensors scattered around the city and collecting large amount of data regarding the surrounding environment. Using multi-key HAs each sensor can authenticate and outsource to the Cloud the data it collects; the Cloud can compute statistics on the authenticated data and provide a central control unit with the result along with a certificate vouching for its correctness.

An important aspect of our definition is a mechanism that allows the verifier to keep track of the users that authenticated the inputs of $\mathcal{P}$, i.e., to know which user contributed to each input wire of $\mathcal{P}$. To formalize this mechanism, we build 
on the model of labeled data and programs of Gennaro and Wichs [70] (we refer the reader to Section 4.4 for details). In terms of security, multi-key HAs allow the adversary to corrupt users (i.e., to learn their secret keys); yet this knowledge should not help the adversary in tampering with the results of programs which involve inputs of honest (i.e., uncorrupted) users only. Our model allows to handle compromised users in a similar way to what occurs with classical digital signatures: a compromised user could be banned by means of a certificate revocation, and could easily be re-integrated via a new key pair 1 Thinking of the sensor network application, if a sensor in the field gets compromised, the data provided by other sensors remains secure, and a new sensor can be easily introduced in the system with new credentials.

Finally, we require multi-key homomorphic authenticators to be succinct in the sense that the size of authenticators is bounded by some fixed polynomial in $(\lambda, n, \log t)$, where $\lambda$ is the security parameter, $n$ is the number of users contributing to the computation and $t$ is the total number of inputs of $\mathcal{P}$. Although such dependence on $n$ may look undesirable, we stress that it is still meaningful in many application scenarios where $n$ is much smaller than $t$. For instance, in the application scenario of healthcare institutions that we mentioned in the introduction, a few hospitals can provide a large amount of data from patients.

\subsection{An Overview of Our Contribution}

A Multi-Key Homomorphic Signature for All Circuits. After setting the definition of multi-key homomorphic authenticators, we proceed to construct multi-key HA schemes. Our first contribution is a multi-key homomorphic signature that supports the evaluation of boolean circuits of depth bounded by a fixed polynomial in the security parameter. The scheme is proven secure based on the small integer solution (SIS) problem over standard lattices [98], and tolerates adversaries that corrupt users non-adaptively $\left.\right|^{2}$ Our technique is inspired by the ones developed by Gorbunov, Vaikuntanathan and Wichs [77] to construct a (single-key) homomorphic signature. Our key contribution is on providing a new representation of the signatures that enables to homomorphically compute over them even if they were generated using different keys. Furthermore, our scheme enjoys an additional property, not fully satisfied by [77]: every user can authenticate separately every data item $m_{i}$ of a collection $m_{1}, \ldots m_{t}$, and the correctness of computations is guaranteed even when computing on not-yet-full datasets. Although it is possible to modify the scheme in [77] for signing data items separately, the security would only work against adversaries that query the

\footnotetext{
${ }^{1}$ Here we mean that this process does not add more complications than the ones already existing for classical digital signatures (e.g., relying on PKI mechanisms).

${ }^{2}$ Precisely, our "core" scheme is secure against adversaries that make non-adaptive signing queries; this is upgraded to adaptive security via general transformations.
} 
whole dataset. In contrast, we prove our scheme to be secure under a stronger security definition where the adversary can adaptively query the various data items, and it can try to cheat by pretending to possess signatures on data items that it never queried (so-called Type 3 forgeries). We highlight that the scheme in [77] is not secure under the stronger definition (with Type 3 forgeries) used in this paper, and we had to introduce new techniques to deal with this scenario. This new property is particularly interesting as it enables users to authenticate and outsource data items in a streaming fashion, without ever having to store the whole dataset. This is useful in applications where the dataset size can be very large or not fixed a priori.

A Multi-Key Homomorphic MAC for Low-Degree Circuits. Our second construction is a multi-key homomorphic MAC that supports the evaluation of arithmetic circuits whose degree $d$ is at most polynomial in the security parameter, and whose inputs come from a small number $n$ of users. For results of such computations the corresponding authenticators have at most size $\left.s=\left(\begin{array}{c}n+d \\ d\end{array}\right)\right]^{3}$ Notably, the authenticator's size is completely independent of the total number of inputs of the arithmetic circuit. Compared to our multi-key homomorphic signature, this construction is only secretly verifiable (i.e., Bob has to know the secret verification keys of all users involved in the computation) and supports a class of computations that is less expressive; also its succinctness is asymptotically worse. In spite of these drawbacks, our multi-key homomorphic MAC achieves interesting features. From the theoretical point of view, it is based on very simple cryptographic tools: a family of pseudorandom functions. Thus, the security relies only on one-way functions. On the practical side, it is particularly efficient: generating a MAC requires only one pseudo-random function evaluation and a couple of field operations; homomorphic computations boil down to additions and multiplications over a multi-variate polynomial ring $\mathbb{F}_{p}\left[X_{1}, \ldots, X_{n}\right]$.

\subsubsection{Related Work}

Homomorphic MACs and Signatures. As we have already mentioned, homomorphic authenticators have received a lot of attention in previous work focusing either on homomorphic signatures (publicly verifiable) or on homomorphic MACs (private verification with a secret key). Apart from the remarkable advances that we already mentioned with respect to homomorphic signatures, some important results have been also achieved in the area of homomorphic MACs. Gennaro and Wichs [70] have proposed a fully homomorphic MAC based on fully homomorphic encryption. However, their scheme is not secure in the presence of verification queries. More efficient schemes have been proposed later [43, 15, 44] that are secure in the presence of verification queries and are more efficient at the price of supporting only restricted homomorphisms. Moreover, we note that

\footnotetext{
${ }^{3}$ Note that $s$ can be bounded by $\operatorname{poly}(n)$ for constant $d$, or by $\operatorname{poly}(d)$ for constant $n$.
} 
Agrawal et al. 44] considered a notion of multi-key signatures for network coding, and proposed a solution which works for linear functions only. Compared to this work, our contribution shows a full-fledged framework for multi-key homomorphic authenticators, and provides solutions that address a more expressive class of computations.

Verifiable Computation. Achieving correctness of outsourced computations is also the aim of verifiable delegation of computation (VC) [75, 67, 52, 22, 103, 62, In this setting, a client wants to delegate the computation of a function $f$ on input $x$ to an untrusted cloud-server. If the server replies with $y$, the client's goal is to verify the correctness of $y=f(x)$ spending less resources than those needed to execute $f$. As mentioned in previous work (e.g., [70, 77]) a crucial difference between verifiable computation and homomorphic authenticators is that in VC the verifier has to know the input of the computation - which can be huge whereas in HAs one can verify by only knowing the function $f$ and the result $y$. Moreover, although some results of verifiable computation could be re-interpreted to solve scenarios similar to the ones addressed by HAs, results based on VC would still present several limitations. For instance, using homomorphic authenticators the server can prove correctness of $y=f(x)$ with a single message, without needing any special encoding of $f$ from the delegator. Second, HAs come naturally with a composition property which means that the outputs of some computations on authenticated data (which is already authenticated) can be fed as input for follow-up computations. This feature is of particular interest to parallelize and or distribute computations (e.g., MapReduce). Emulating this composition within $\mathrm{VC}$ systems is possible by means of certain non-interactive proof systems [23] but leads to complex statements and less natural realizations. A last advantage is that by using HAs, clients can authenticate various (small) pieces of data independently and without storing previously outsourced data. In contrast, most VC systems require clients to encode the whole input in 'one shot', and often such encoding can be used in a single computation only.

Multi-Client Verifiable Computation. Another line of work, closely related to ours is that on multi-client verifiable computation [51, 78]. This primitive, introduced by Choi et al. [51], aims to extend VC to the setting where inputs are provided by multiple users, and one of these users wants to verify the result's correctness.

Choi et al. [51] proposed a definition and a multi-client VC scheme which generalizes that of Gennaro et al. [67].

The solution in [51], however, does not consider malicious or colluding clients. This setting was addressed by Gordon et al. in [78], where they provide a scheme with stronger security guarantees against a malicious server or an arbitrary set of malicious colluding clients.

It is interesting to notice that in the definition of multi-client $\mathrm{VC}$ all the clients but the one who verifies can encode inputs independently of the function to be 
later executed on them. One may thus think that the special case in which the verifier provides no input yields a solution similar to the one achieved by multikey HAs. However, a closer look at the definitions and the existing constructions of multi-client VC reveals three main differences. (1) In multi-client VC, in order to prove the correctness of an execution of a function $f$, the server has to wait a message from the verifier which includes some encoding of $f$. This is not necessary in multi-key HAs where the server can directly prove the correctness of $f$ on previously authenticated data with a single message and without any function's encoding. (2) The communication between the server and the verifier is at least linear in the total number of inputs of $f$ : this can be prohibitive in the case of computations on very large inputs (think of TBytes of data). In contrast, with multi-key HAs the communication between the server and the verifier is proportional only to the number of users, and depends only logarithmically on the total number of inputs. (3) In multi-client VC an encoding of one input can be used in a single computation. Thus, if a user wants to first upload data on the server to later execute many functions on it, then the user has to provide as many encodings as the number of functions to be executed. In contrast, multi-key HAs allow one to encode (i.e., authenticate) every input only once and to use it for proving correctness of computations an unbounded number of times.

\subsection{Preliminaries}

We collect here some basic definitions used throughout this chapter.

Definition 15 (Statistical Distance). Let $X, Y$ denote two random variables with support $\mathcal{X}, \mathcal{Y}$ respectively; the statistical distance between $X$ and $Y$ is defined as

$$
\operatorname{SD}(X, Y):=\frac{1}{2} \sum_{u \in \mathcal{X} \cup \mathcal{Y}}|\operatorname{Pr}[X=u]-\operatorname{Pr}[Y=u]| .
$$

If $\mathbf{S D}(X, Y)=\operatorname{negl}(\lambda)$, we say that $X$ and $Y$ are statistically close and we write $X \stackrel{\text { stat }}{\approx} Y$.

Definition 16 (Entropy [57]). The min-entropy of a random variable $X$ is defined as

$$
\mathbf{H}_{\infty}(X):=-\log \left(\max _{x} \operatorname{Pr}[X=x]\right) .
$$

The (average-) conditional min-entropy of a random variable $X$ conditioned on a correlated variable $Y$ is defined as

$$
\mathbf{H}_{\infty}(X \mid Y):=-\log \left(\underset{y \leftarrow Y}{\mathbf{E}}\left[\max _{x} \operatorname{Pr}[X=x \mid Y=y]\right]\right) .
$$

The optimal probability of an unbounded adversary guessing $X$ when given a correlated value $Y$ is $2^{-\mathbf{H}_{\infty}(X \mid Y)}$.

Lemma 20 ([57]). Let $X, Y$ be arbitrarily random variables where the support of $Y$ lies in $\mathcal{Y}$. Then $\mathbf{H}_{\infty}(X \mid Y) \geq \mathbf{H}_{\infty}(X)-\log (|\mathcal{Y}|)$. 


\subsection{Multi-Key Homomorphic Authenticators}

In this section, we present our new notion of Multi-Key Homomorphic Authenticators (multi-key HAs). Intuitively, multi-key HAs extend the existing notions of homomorphic signatures [31] and homomorphic MACs [70] in such a way that one can homomorphically compute a program $\mathcal{P}$ over data authenticated using different secret keys. For the sake of verification, in multi-key HAs the verifier needs to know the verification keys of all users that provided inputs to $\mathcal{P}$. Our definitions are meant to be general enough to be easily adapted to both the case in which verification keys are public and the one where verification keys are secret. In the former case, we call the primitive multi-key homomorphic signatures whereas in the latter case we call it multi-key homomorphic MACs.

As already observed in previous work about HAs, it is important that an authenticator $\sigma_{\mathcal{P}, y}$ does not authenticate a value $y$ out of context, but only as the output of a program $\mathcal{P}$ executed on previously authenticated data. To formalize this notion, we build on the model of labeled data and programs of Gennaro and Wichs [70]. The idea of this model is that every data item is authenticated under a unique label $\ell$. For example, in scenarios where the data is outsourced, such labels can be thought of as a way to index/identify the remotely stored data. A labeled program $\mathcal{P}$, on the other hand, consists of a circuit $f$ where every input wire $i$ has a label $\ell_{i}$. Going back to the outsourcing example, a labeled program is a way to specify on what portion of the outsourced data one should execute a circuit $f$. More formally, the notion of labeled programs of [70] is recalled below.

Labeled Programs [70]. A labeled program $\mathcal{P}$ is a tuple $\left(f, \ell_{1}, \ldots, \ell_{n}\right)$, such that $f: \mathcal{M}^{n} \rightarrow \mathcal{M}$ is a function of $n$ variables (e.g., a circuit) and $\ell_{i} \in\{0,1\}^{*}$ is a label for the $i$-th input of $f$. Labeled programs can be composed as follows: given $\mathcal{P}_{1}, \ldots, \mathcal{P}_{t}$ and a function $g: \mathcal{M}^{t} \rightarrow \mathcal{M}$, the composed program $\mathcal{P}^{*}$ is the one obtained by evaluating $g$ on the outputs of $\mathcal{P}_{1}, \ldots, \mathcal{P}_{t}$, and it is denoted as $\mathcal{P}^{*}=g\left(\mathcal{P}_{1}, \ldots, \mathcal{P}_{t}\right)$. The labeled inputs of $\mathcal{P}^{*}$ are all the distinct labeled inputs of $\mathcal{P}_{1}, \ldots \mathcal{P}_{t}$ (all the inputs with the same label are grouped together and considered as a unique input of $\mathcal{P}^{*}$ ). Let $f_{\text {id }}: \mathcal{M} \rightarrow \mathcal{M}$ be the identity function and $\ell \in\{0,1\}^{*}$ be any label. We refer to $\mathcal{I}_{\ell}=\left(f_{i d}, \ell\right)$ as the identity program with label $\ell$. Note that a program $\mathcal{P}=\left(f, \ell_{1}, \ldots, \ell_{n}\right)$ can be expressed as the composition of $n$ identity programs $\mathcal{P}=f\left(\mathcal{I}_{\ell_{1}}, \ldots, \mathcal{I}_{\ell_{n}}\right)$.

Using labeled programs to identify users. In our notion of multi-key homomorphic authenticators, one wishes to verify the outputs of computations executed on data authenticated under different keys. A meaningful definition of multi-key HAs thus requires that the authenticators are not out of context also with respect to the set of keys that contributed to the computation. To address this issue, we assume that every user has an identity id in some identity space ID, and that the user's keys are associated to id by means of any suitable mechanism (e.g., PKI). Next, in order to distinguish among data of different users and to 
identify to which input wires a certain user contributed, we assume that the label space contains the set ID. Namely, in our model a data item is assigned a label $\ell:=(\mathrm{id}, \tau)$, where id is a user's identity, and $\tau$ is a tag; this essentially identifies uniquely a data item of user id with index $\tau$. For compatibility with previous notions of homomorphic authenticators, we assume that data items can be grouped in datasets, and one can compute only on data within the same dataset. In our definitions, a dataset is identified by an arbitrary string $\Delta .4$

Definition 17 (Multi-Key Homomorphic Authenticator). A multi-key homomorphic authenticator scheme MKHAut consists of a tuple of PPT algorithms (Setup, KeyGen, Auth, Eval, Ver) satisfying the following properties: authentication correctness, evaluation correctness, succinctness and security. The five algorithms work as follows:

$\operatorname{Setup}\left(1^{\lambda}\right)$. The setup algorithm takes as input the security parameter $\lambda$ and outputs some public parameters $\mathrm{pp}$. These parameters include (at least) descriptions of a tag space $\mathcal{T}$, an identity space ID, the message space $\mathcal{M}$ and a set of admissible functions $\mathbb{F}$. Given $\mathcal{T}$ and ID, the label space of the scheme is defined as their cartesian product $\mathcal{L}:=\mathrm{ID} \times \mathcal{T}$. For a labeled program $\mathcal{P}=\left(f, \ell_{1}, \ldots, \ell_{t}\right)$ with labels $\ell_{i}:=\left(\mathrm{id}_{i}, \tau_{i}\right) \in \mathcal{L}$, we use id $\in \mathcal{P}$ as compact notation for $\mathrm{id} \in\left\{\mathrm{id}_{1}, \ldots, \mathrm{id}_{t}\right\}$. The $\mathrm{pp}$ are input to all the following algorithms, even when not specified.

KeyGen(pp). The key generation algorithm takes as input the public parameters and outputs a triple of keys (ak, ek, vk), where ak is a secret authentication key, ek is a public evaluation key and $\mathrm{vk}$ is a verification key which could be either public or private..$^{5}$

Auth(ak, $\Delta, \ell, \mathrm{m})$. The authentication algorithm takes as input an authentication key ak, a dataset identifier $\Delta$, a label $\ell=(\mathrm{id}, \tau)$ for the message $\mathrm{m}$, and it outputs an authenticator $\sigma$.

$\operatorname{Eval}\left(f,\left\{\left(\sigma_{i}, \mathrm{EKS}_{i}\right)\right\}_{i \in[t]}\right)$. The evaluation algorithm takes as input a $t$-input function $f: \mathcal{M}^{t} \longrightarrow \mathcal{M}$, and a set $\left\{\left(\sigma_{i}, \mathrm{EKS}_{i}\right)\right\}_{i \in[t]}$ where each $\sigma_{i}$ is an authenticator and each $\mathrm{EKS}_{i}$ is a set of evaluation keys. ${ }^{6}$

$\operatorname{Ver}\left(\mathcal{P}, \Delta,\left\{\mathrm{vk}_{\mathrm{id}}\right\}_{\mathrm{id} \in \mathcal{P}}, \mathrm{m}, \sigma\right)$. The verification algorithm takes as input a labeled program $\mathcal{P}=\left(f, \ell_{1}, \ldots, \ell_{t}\right)$, a dataset identifier $\Delta$, the set of verification

\footnotetext{
${ }^{4}$ Although considering the dataset notion complicates the definition, it also provides some benefits, as we illustrate later in the constructions. For instance, when verifying for the same program $\mathcal{P}$ over different datasets, one can perform some pre-computation that makes further verifications cheap.

${ }^{5}$ As mentioned earlier, the generated triple (ak, ek, vk) will be associated to an identity id $\in$ ID. When this connection becomes explicit, we will refer to (ak, ek, vk) as $\left(s k_{i d}, e k_{i d}, v k_{i d}\right)$.

${ }^{6}$ The motivation behind the evaluation-keys set $\mathrm{EKS}_{i}$ is that, if $\sigma_{i}$ authenticates the output of a labeled program $\mathcal{P}_{i}$, then $\mathrm{EKS}_{i}=\left\{\mathrm{ek}_{\mathrm{id}}\right\}_{\mathrm{id} \in \mathcal{P}_{i}}$ should be the set of evaluation keys corresponding to identities involved in the computation of $\mathcal{P}_{i}$.
} 
keys $\left\{\mathrm{vk}_{\mathrm{id}}\right\}_{\mathrm{id} \in \mathcal{P}}$ corresponding to those identities id involved in the program $\mathcal{P}$, a message $\mathrm{m}$ and an authenticator $\sigma$. It outputs 0 (reject) or 1 (accept).

Authentication Correctness. Intuitively, a Multi-Key Homomorphic Autenticator has authentication correctness if the output of Auth(ak, $\Delta, \ell, \mathrm{m})$ verifies correctly for $\mathrm{m}$ as the output of the identity program $\mathcal{I}_{\ell}$ over the dataset $\Delta$. More formally, a scheme MKHAut satisfies authentication correctness if for all public parameters $\mathrm{pp} \leftarrow \operatorname{Setup}\left(1^{\lambda}\right)$, any key triple $(\mathrm{ak}, \mathrm{ek}, \mathrm{vk}) \leftarrow \operatorname{KeyGen}(\mathrm{pp})$, any label $\ell=(\mathrm{id}, \tau) \in \mathcal{L}$ and any authenticator $\sigma \leftarrow$ Auth(ak, $\Delta, \ell, \mathrm{m})$, we have $\operatorname{Ver}\left(\mathcal{I}_{\ell}, \Delta, \mathrm{vk}, \mathrm{m}, \sigma\right)$ outputs 1 with all but negligible probability.

Evaluation Correctness. Intuitively, this property says that running the evaluation algorithm on signatures $\left(\sigma_{1}, \ldots, \sigma_{t}\right)$ such that each $\sigma_{i}$ verifies for $\mathrm{m}_{i}$ as the output of a labeled program $\mathcal{P}_{i}$ over the dataset $\Delta$, it produces a signature $\sigma$ which verifies for $f\left(\mathrm{~m}_{1}, \ldots, \mathrm{m}_{t}\right)$ as the output of the composed program $f\left(\mathcal{P}_{1}, \ldots, \mathcal{P}_{t}\right)$ over the dataset $\Delta$. More formally, let us fix the public parameters pp $\leftarrow \operatorname{Setup}\left(1^{\lambda}\right)$, a set of key triples $\left\{\left(\mathrm{sk}_{\text {id }}, \mathrm{ek}_{\mathrm{id}}, \mathrm{vk_{ \text {id } }}\right)\right\}_{\text {id } \in \text { ID }}$ for some ID $\subseteq \mathrm{ID}$, a dataset $\Delta$, a function $g: \mathcal{M}^{t} \rightarrow \mathcal{M}$, and any set of program/message/authenticator triples $\left\{\left(\mathcal{P}_{i}, m_{i}, \sigma_{i}\right)\right\}_{i \in[t]}$ such that $\operatorname{Ver}\left(\mathcal{P}_{i}, \Delta,\left\{\mathrm{vk}_{\mathrm{id}}\right\}_{\mathrm{id} \in \mathcal{P}_{i}}, \mathrm{~m}_{i}, \sigma_{i}\right)=1$ for all $i \in[t]$. Let $\mathrm{m}^{*}=g\left(\mathrm{~m}_{1}, \ldots, \mathrm{m}_{t}\right), \mathcal{P}^{*}=g\left(\mathcal{P}_{1}, \ldots, \mathcal{P}_{t}\right)$, and $\sigma^{*}=\operatorname{Eval}\left(g,\left\{\left(\sigma_{i}\right.\right.\right.$, $\left.\left.\left.\mathrm{EKS}_{i}\right)\right\}_{i \in[t]}\right)$ where $\mathrm{EKS}_{i}=\left\{\mathrm{ek}_{\mathrm{id}}\right\}_{\mathrm{id} \in \mathcal{P}_{i}}$. Then, $\operatorname{Ver}\left(\mathcal{P}^{*}, \Delta,\left\{\mathrm{vk}_{\mathrm{id}}\right\}_{\mathrm{id} \in \mathcal{P}^{*}}, \mathrm{~m}^{*}, \sigma^{*}\right)=1$ holds with all but negligible probability.

Succinctness. A multi-key HA is said to be succinct if the size of every authenticator depends only logarithmically on the size of a dataset. However, we allow authenticators to depend on the number of keys involved in the computation. More formally, let $\mathrm{pp} \leftarrow \operatorname{Setup}\left(1^{\lambda}\right), \mathcal{P}=\left(f, \ell_{1}, \ldots, \ell_{t}\right)$ with $\ell_{i}=\left(\mathrm{id}_{i}, \tau_{i}\right)$, $\left\{\left(\mathrm{sk}_{\mathrm{id}}, \mathrm{ek}_{\mathrm{id}}, \mathrm{vk}_{\mathrm{id}}\right) \leftarrow \operatorname{KeyGen}(\mathrm{pp})\right\}_{\mathrm{id} \in \mathcal{P}}$, and $\sigma_{i} \leftarrow \operatorname{Auth}\left(\mathrm{ak}_{\mathrm{id}_{i}}, \Delta, \ell_{i}, \mathrm{~m}_{i}\right)$ for all $i \in[t]$. A multi-key HA is said to be succinct if there exists a fixed polynomial $p$ such that $|\sigma|=p(\lambda, n, \log t)$ where $\sigma=\operatorname{Eval}\left(g,\left\{\left(\sigma_{i}, \mathrm{ek}_{\mathrm{id}_{i}}\right)\right\}_{i \in[t]}\right)$ and $n=|\{\mathrm{id} \in \mathcal{P}\}|$.

Remark 4. Succinctness is one of the crucial properties that make multi-key HAs an interesting primitive. Without succinctness, a trivial multi-key HA construction is the one where Eval outputs the concatenation of all the signatures (and messages) given in input, and the verifier simply checks each message-signature pair and recomputes the function by himself. Our definition of succinctness, where signatures can grow linearly with the number of keys but logarithmically in the total number of inputs, is also non-trivial, especially when considering settings where there are many more inputs than keys (in which case, the above trivial construction would not work). Another property that can make homomorphic signatures an interesting primitive is privacy-context-hiding-as considered in prior work. Intuitively, context-hiding guarantees that signatures do not reveal information on the original inputs. While we leave the study of context-hiding for multi-key HAs for future work, we note that a trivial construction that is context-hiding but not succinct can be easily obtained with the additional help of non-interactive 
zero-knowledge proofs of knowledge: the idea is to extend the trivial construction above by requiring the evaluator to generate a NIZK proof of knowledge of valid input messages and signatures that yield the public output. In this sense, we believe that succinctness is the most non-trivial property to achieve in homomorphic signatures, and this is what we focus on in this work.

Security. Intuitively, our security model for multi-key HAs guarantees that an adversary, without knowledge of the secret keys, can only produce authenticators that were either received from a legitimate user, or verify correctly on the results of computations executed on the data authenticated by legitimate users. Moreover, we also give to the adversary the possibility of corrupting users. In this case, it must not be able to cheat on the outputs of programs that get inputs from uncorrupted users only. In other words, our security definition guarantees that the corruption of one user affects the data of that user only, but does not endanger the integrity of computations among the other (un-corrupted) users of the system. We point out that preventing cheating on programs that involve inputs of corrupted users is inherently impossible in multi-key HAs, at least if general functions are considered. For instance, consider an adversary who picks the function $\left(x_{1}+x_{2}\right.$ $\bmod p$ ) where $x_{1}$ is supposed to be provided by user Alice. If the adversary corrupts Alice, it can use her secret key to inject any input authenticated on her behalf and thus bias the output of the function at its will.

The formalization of the intuitions illustrated above is more involved. For a scheme MKHAut we define security via the following experiment between a challenger $\mathcal{C}$ and an adversary $\mathcal{A}\left(\operatorname{HomUF}_{-C_{A} \text { MKHAut }}(\lambda)\right)$ :

Setup. $\mathcal{C}$ runs Setup $\left(1^{\lambda}\right)$ to obtain the public parameters pp that are sent to $\mathcal{A}$.

Authentication Queries $\mathcal{A}$ can adaptively submit queries of the form $(\Delta, \ell, \mathrm{m})$, where $\Delta$ is a dataset identifier, $\ell=($ id, $\tau)$ is a label in ID $\times \mathcal{T}$ and $\mathrm{m} \in \mathcal{M}$ are messages of his choice. $\mathcal{C}$ answers as follows:

- If $(\Delta, \ell, \mathrm{m})$ is the first query for the dataset $\Delta, \mathcal{C}$ initializes an empty list $L_{\Delta}=\emptyset$ and proceeds as follows.

- If $(\Delta, \ell, \mathrm{m})$ is the first query with identity id, $\mathcal{C}$ generates keys $\left(\mathrm{sk}_{\mathrm{id}}, \mathrm{ek}_{\mathrm{id}}\right.$, $\left.\mathrm{vk}_{\mathrm{id}}\right) \stackrel{\$}{\leftrightarrow}$ KeyGen(pp) (that are implicitly assigned to identity id), gives ek id to $\mathcal{A}$ and proceeds as follows.

- If $(\Delta, \ell, \mathrm{m})$ is such that $(\ell, \mathrm{m}) \notin L_{\Delta}, \mathcal{C}$ computes $\sigma_{\ell} \leftrightarrow \operatorname{Auth}\left(\mathrm{sk}_{\mathrm{id}}, \Delta, \ell, \mathrm{m}\right)$ (note that $\mathcal{C}$ has already generated keys for the identity id), returns $\sigma_{\ell}$ to $\mathcal{A}$, and updates the list $L_{\Delta} \leftarrow L_{\Delta} \cup(\ell, \mathrm{m})$.

- If $\left(\Delta, \ell, \mathrm{m}\right.$ ) is such that $(\ell, \cdot) \in L_{\Delta}$ (which means that the adversary had already made a query $\left(\Delta, \ell, \mathrm{m}^{\prime}\right)$ for some message $\left.\mathrm{m}^{\prime}\right)$, then $\mathcal{C}$ ignores the query. 
Verification Queries $\mathcal{A}$ is also given access to a verification oracle. Namely, the adversary can submit a query $(\mathcal{P}, \Delta, \mathrm{m}, \sigma)$, and $\mathcal{C}$ replies with the output of $\operatorname{Ver}\left(\mathcal{P}, \Delta,\left\{\mathrm{vk}_{\mathrm{id}}\right\}_{\mathrm{id} \in \mathcal{P}}, \mathrm{m}, \sigma\right)$.

Corruption The adversary has access to a corruption oracle. At the beginning of the game, the challenger initialises an empty list $L_{\text {corr }}=\emptyset$ of corrupted identities; during the game, $\mathcal{A}$ can adaptively query identities id $\in$ ID. If id $\notin L_{\text {corr }}$, then $\mathcal{C}$ replies with the triple $\left(\mathrm{sk}_{\mathrm{id}}, \mathrm{ek}_{\mathrm{id}}, \mathrm{vk}_{\mathrm{id}}\right)$ (that is generated using KeyGen if not done before) and updates the list $L_{\text {corr }} \leftarrow L_{\text {corr }} \cup$ id. If id $\in L_{\text {corr }}$, then $\mathcal{C}$ replies with the triple ( $\left.\mathbf{s k}_{\mathrm{id}}, \mathrm{ek}_{\mathrm{id}}, \mathrm{vk}_{\mathrm{id}}\right)$ assigned to id before.

Forgery In the end, $\mathcal{A}$ outputs a tuple $\left(\mathcal{P}^{*}, \Delta^{*}, \mathrm{~m}^{*}, \sigma^{*}\right)$. The experiment outputs 1 if the tuple returned by $\mathcal{A}$ is a forgery (defined below), and 0 otherwise.

Definition 18 (Forgery). Consider an execution of $\operatorname{HomUF-CMA} A_{\mathcal{A}, \mathrm{MKHAut}}(\lambda)$ where $\left(\mathcal{P}^{*}, \Delta^{*}, \mathrm{~m}^{*}, \sigma^{*}\right)$ is the tuple returned by the adversary in the end of the experiment, with $\mathcal{P}^{*}=\left(f^{*}, \ell_{1}^{*}, \ldots, \ell_{t}^{*}\right)$. This is a forgery if $\operatorname{Ver}\left(\mathcal{P}^{*}, \Delta^{*},\left\{\mathrm{vk}_{\mathrm{id}}\right\}_{\mathrm{id} \in \mathcal{P}^{*}}, \mathrm{~m}^{*}\right.$, $\left.\sigma^{*}\right)=1$, for all id $\in \mathcal{P}^{*}$ we have that id $\notin L_{\text {corr }}$ (i.e., no identity involved in $\mathcal{P}^{*}$ is corrupted), and either one of the following properties is satisfied:

Type 1: $L_{\Delta^{*}}$ has not been initialized during the game (i.e., the dataset $\Delta^{*}$ was never queried).

Type 2: For all $i \in[t], \exists\left(\ell_{i}^{*}, \mathrm{~m}_{i}\right) \in L_{\Delta^{*}}$, but $\mathrm{m}^{*} \neq f^{*}\left(\mathrm{~m}_{1}, \ldots, \mathrm{m}_{t}\right)$ (i.e., $\mathrm{m}^{*}$ is not the correct output of $\mathcal{P}^{*}$ when executed over previously authenticated messages).

Type 3: There exists a label $\ell^{*}$ such that $\left(\ell^{*}, \cdot\right) \notin L_{\Delta^{*}}($ i.e., $\mathcal{A}$ never made a query with label $\ell^{*}$ ).

We say that a HA scheme MKHAut is secure if for every PPT adversary $\mathcal{A}$, its advantage $\operatorname{Adv}_{\text {MKHAut }, \mathcal{A}}^{\text {HomU }}(\lambda)=\operatorname{Pr}\left[\operatorname{HomUF-CMA} \mathcal{A}\right.$, MKHAut $_{(\lambda)=1] \text { is negligible. }}$

Remark 5 (Comparison with previous security definitions). Our security notion can be seen as the multi-key version of the one proposed by Gennaro and Wichs in [70] (in their model our Type 3 forgeries are called 'Type 1' as they do not consider multiple datasets). We point out that even in the special case of a single key, our security definition is stronger than the ones used in previous work [31, 63, 49, 777] (with the only exception of [70]). The main difference lies in our definition of Type 3 forgeries. The intuitive idea of this kind of forgeries is that an adversary who did not receive an authenticated input labeled by a certain $\ell^{*}$ cannot produce a valid authenticator for the output of a computation which has $\ell^{*}$ among its inputs. In [31, 777] these forgeries were not considered at all, as the adversary is assumed to query the dataset always in full. Other works [63, 43, 49] consider a weaker Type 3 notion, which deals with the concept of "well defined programs", where the input wire labeled by the missing label $\ell^{*}$ is required to "contribute" to 
the computation (i.e., it must change its outcome). The issue with such a Type 3 definition is that it may not be efficient to test if an input contributes to a function, especially if the admissible functions are general circuits. In contrast our definition above is simpler and efficiently testable since it simply considers a Type 3 forgery one where the labeled program $\mathcal{P}^{*}$ involves an un-queried input.

Multi-Key Homomorphic Signatures. As previously mentioned, our definitions are general enough to be easily adapted to either case in which verification is secret or public. The only difference is whether the adversary is allowed to see the verification keys in the security experiment. When the verification is public, we call the primitive multi-key homomorphic signatures. More formally:

Definition 19 (Multi-Key Homomorphic Signatures). A multi-key homomorphic signature is a multi-key homomorphic authenticator in which verification keys are also given to the adversary in the security experiment.

Note that making verification keys public also allows to slightly simplify the security experiment by removing the verification oracle (the adversary can run the verification by itself). In the sequel, when referring to multi-key homomorphic signatures we adapt our notation to the typical one of digital signatures, namely we denote $\operatorname{Auth}(\mathrm{ak}, \Delta, \ell, \mathrm{m})$ as $\operatorname{Sign}(\mathrm{ak}, \Delta, \ell, \mathrm{m})$, and call its outputs signatures.

\subsubsection{Non-Adaptive Corruption Queries}

In our work, we consider a relaxation of the security definition in which the adversaries ask for corruptions in a non-adaptive way. More precisely, we say that an adversary $\mathcal{A}$ makes non-adaptive corruption queries if for every identity id asked to the corruption oracle, id was not queried earlier in the game to the authentication oracle or the verification oracle. For this class of adversaries, it is easy to see that corruption queries are essentially of no help as the adversary can generate keys on its own. More precisely, we can prove the following proposition.

Proposition 2. MKHAut is secure against adversaries that do not make corruption queries if and only if MKHAut is secure against adversaries that make non-adaptive corruption queries.

Proof. The proof is rather simple and we give here only a proof sketch. It is obvious to see that if a scheme is secure in the presence of non-adaptive corruptions, then the same scheme is secure when there is no corruption at all. For the opposite direction, we can show that for every adversary $\mathcal{A}_{\text {corr }}$ that breaks the security of MKHAut by non-adaptive corruptions there is an adversary $\mathcal{B}$ that breaks the security of MKHAut in a game without corruptions. In particular, the reduction is tight, i.e., the success probability of $\mathcal{B}$ is the same as that of $\mathcal{A}_{\text {Corr }}$.

$\mathcal{B}$ simply runs the adversary $\mathcal{A}_{\text {Corr }}$ and forwards all the queries of $\mathcal{A}_{\text {Corr }}$ (except corruption queries) to its challenger. For every corruption query id, $\mathcal{B}$ generates 
a tuple $\left(\mathrm{sk}_{\text {id }}, \mathrm{ek}_{\text {id }}, \mathrm{vk}_{\mathrm{id}}\right) \stackrel{\$}{\leftarrow}$ KeyGen $(\mathrm{pp})$ and returns the tuple to $\mathcal{A}_{\text {Corr }}$. If $\mathcal{A}_{\text {Corr }}$ comes up with a valid forgery, by definition this forgery does not involve any corrupted identity. Hence, the same forgery will be valid also in the game played by $\mathcal{B}$. It is not hard to see that the simulation is perfect since $\mathcal{A}_{\text {Corr }}$ only makes corruption queries for identities that were never asked to the authentication or verification oracles earlier in the game. Therefore, if $\mathcal{A}_{\text {corr }}$ had advantage $\epsilon$ in breaking the scheme in the game with non-adaptive corruptions, then $\mathcal{B}$ has the same advantage to break the scheme without making corruptions at all.

\subsubsection{Weakly-Adaptive Secure multi-key HAs}

In our work, we also consider a weaker notion of security for multi-key HAs in which the adversary has to declare all the queried messages at the beginning of the experiment. More precisely, we consider a notion in which the adversary declares only the messages and the respective tags that will be queried, for every dataset and identity, without, however, needing to specify the names of the datasets or of the identities. In a sense, the adversary $\mathcal{A}$ is adaptive on identities and dataset names, but not on tags and messages. The definition is inspired by the one, for the single-key setting, of Catalano et al. [49].

To define the notion of weakly-adaptive security for multi-key HAs, we intro-

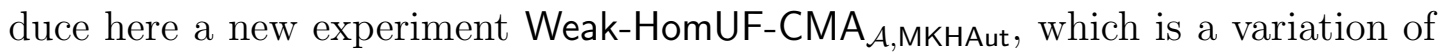
experiment HomUF-CMA $\mathcal{A}_{\mathcal{A} \text { MKHAut }}$ (Definition 17) as described below.

Definition 20 (Weakly-Secure Multi-Key Homomorphic Authenticators). In the security experiment Weak-HomUF-CMA ${ }_{\mathcal{A}, \mathrm{MKHAut}}$, before the setup phase, the adversary $\mathcal{A}$ sends to the challenger $\mathcal{C}$ a collection of sets of tags $\mathcal{T}_{i, k} \subseteq \mathcal{T}$ for $i \in\left[Q_{\mathrm{id}}\right]$ and $k \in\left[Q_{\Delta}\right]$, where $Q_{\mathrm{id}}$ and $Q_{\Delta}$ are, respectively, the total numbers of distinct identities and datasets that will be queried during the game. Associated to every set $\mathcal{T}_{i, k}, \mathcal{A}$ also sends a set of messages $\left\{m_{\tau}\right\}_{\tau \in \mathcal{T}_{i, k}}$. Basically the adversary declares, prior to key generation, all the messages and tags that it will query later on; however $\mathcal{A}$ is not required to specify identity and dataset names. Next, the adversary receives the public parameters from $\mathcal{C}$ and can start the query-phase.

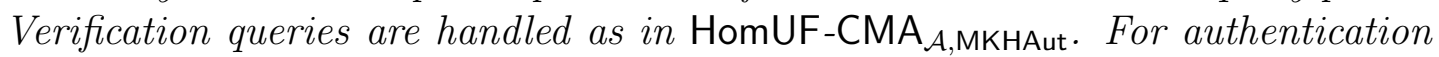
queries, $\mathcal{A}$ can adaptively submit pairs (id, $\Delta)$ to $\mathcal{C}$. The challenger then replies with a set of authenticators $\left\{\sigma_{\tau}\right\}_{\tau \in \mathcal{T}_{i, k}}$, where indices $i, k$ are such that id is the $i$-th queried identity, and $\Delta$ is the $k$-th queried dataset.

An analogous security definition of weakly-secure multi-key homomorphic signatures is trivially obtained by removing a verification oracle.

In the Section 4.5 .4 we present two generic transformations that turn weakly secure multi-key homomorphic authenticator schemes into adaptive secure ones. Our first transformation holds in the standard model and works for schemes in which the tag space $\mathcal{T}$ has polynomial size, while the second one avoids this limitation on the size of $\mathcal{T}$ but holds in the random oracle model. 


\subsection{Our Multi-Key Fully Homomorphic Signa- ture}

In this section, we present our construction of a multi-key homomorphic signature scheme that supports the evaluation of arbitrary circuits of bounded polynomial depth. The scheme is based on the SIS problem on standard lattices, a background of which is provided in the next section. Precisely, in Section 4.5.2 we present a scheme that is weakly-secure and supports a single dataset. Later, in Section 4.5 .3 we discuss how to extend the scheme to handle multiple datasets, whereas the support of adaptive security can be obtained via the applications of our transformations in Section 4.5.4.

\subsubsection{Lattices and Small Integer Solution Problem}

We recall here notation and some basic results about lattices that are useful to describe our homomorphic signature construction.

For any positive integer $q$ we denote by $\mathbb{Z}_{q}$ the ring of integers modulo $q$. Elements in $\mathbb{Z}_{q}$ are represented as integers in the range $\left(-\frac{q}{2}, \frac{q}{2}\right]$. The absolute value of any $x \in \mathbb{Z}_{q}$ (denoted with $|x|$ ) is defined by taking the modulus $q$ representative of $x$ in $\left(-\frac{q}{2}, \frac{q}{2}\right]$, i.e., take $y=x \bmod q$ and then set $|x|=|y| \in\left[0, \frac{q}{2}\right]$. Vectors and matrices are denoted in bold. For any vector $\mathbf{u}:=\left(u_{i}, \ldots, u_{n}\right) \in \mathbb{Z}_{q}^{n}$, its infinity norm is $\|\mathbf{u}\|_{\infty}:=\max _{i \in[n]}\left|u_{i}\right|$, and similarly for a matrix $\mathbf{A}:=\left[a_{i, j}\right] \in \mathbb{Z}_{q}^{n \times m}$ we write $\|\mathbf{A}\|_{\infty}:=\max _{i \in[n], j \in[m]}\left|a_{i, j}\right|$.

The Small Integer Solution Problem (SIS). For integer parameters $n, m, q$ and $\beta$, the $\operatorname{SIS}(n, m, q, \beta)$ problem provides to an adversary $\mathcal{A}$ a uniformly random matrix $\mathbf{A} \in \mathbb{Z}_{q}^{n \times m}$, and requires $\mathcal{A}$ to find a vector $\mathbf{u} \in \mathbb{Z}_{q}^{n}$ such that $\mathbf{u} \neq \mathbf{0}$, $\|\mathbf{u}\|_{\infty} \leq \beta$, and $\mathbf{A} \cdot \mathbf{u}=\mathbf{0}$. More formally,

Definition 21 (SIS [98]). Let $\lambda \in \mathbb{N}$ be the security parameter. For values $n=$ $n(\lambda), m=m(\lambda), q=q(\lambda), \beta=\beta(\lambda)$, defined as functions of $\lambda$, the $\operatorname{SIS}(n, m, q, \beta)$ hardness assumption holds if for any PPT adversary $\mathcal{A}$ we have

$$
\operatorname{Pr}\left[\mathbf{A} \cdot \mathbf{u}=\mathbf{0} \wedge \mathbf{u} \neq \mathbf{0} \wedge\|\mathbf{u}\|_{\infty} \leq \beta: \mathbf{A} \stackrel{\$}{\leftarrow} \mathbb{Z}_{q}^{n \times m}, \mathbf{u} \leftarrow \mathcal{A}\left(1^{\lambda}, \mathbf{A}\right)\right] \leq \operatorname{neg} \mid(\lambda) .
$$

For standard lattices, the SIS problem is known to be as hard as solving certain worst-case instances of lattice problems [5, 95, 98, 97], and is also implied by the hardness of learning with error (we refer any interested reader to the cited papers for the technical details about the parameters).

In our work, we assume that for any $\beta=2^{\text {poly }(\lambda)}$ there are some $n=\operatorname{poly}(\lambda)$, $q=2^{\text {poly }(\lambda)}$, with $q>\beta$, such that for all $m=\operatorname{poly}(\lambda)$ the $\operatorname{SIS}(n, m, q, \beta)$ hardness assumption holds. This parameters choice assures that hardness of worst-case lattice problems holds with sub-exponential approximation factors.

Trapdoors for Lattices. The SIS problem is hard to solve for a random matrix A. However, there is a way to sample a random $\mathbf{A}$ together with a trapdoor such 
that SIS becomes easy to solve for that $\mathbf{A}$, given the trapdoor. Additionally, it has been shown that there exist "special" (non random) matrices G for which SIS is easy to solve as well. The following lemma summarizes the above known results (similar to a lemma in [33]):

Lemma 21 ([6, 72, 7, 96]). There exist efficient algorithms TrapGen, SamPre, Sam such that the following holds: given integers $n \geq 1, q \geq 2$, there exist some $m^{*}=m^{*}(n, q)=O(n \log q), \beta_{\text {sam }}=\beta_{\text {sam }}(n, q)=O(n \sqrt{\log q})$ such that for all $m \geq m^{*}$ and all $k$ (polynomial in $n$ ) we have:

1 Sam $\left(1^{m}, 1^{k}, q\right) \rightarrow \mathbf{U}$ samples a matrix $\mathbf{U} \in \mathbb{Z}_{q}^{m \times k}$ such that $\|\mathbf{U}\|_{\infty} \leq \beta_{\text {sam }}$ (with probability 1 ).

2 For $(\mathbf{A}, \mathrm{td}) \leftarrow \operatorname{TrapGen}\left(1^{n}, 1^{m}, q\right), \mathbf{A}^{\prime} \stackrel{\$}{\leftarrow} \mathbb{Z}_{q}^{n \times m}, \mathbf{U} \leftarrow \operatorname{Sam}\left(1^{m}, 1^{k}, q\right), \mathbf{V}:=$ $\mathbf{A U}, \mathbf{V}^{\prime} \stackrel{\$}{\leftarrow} \mathbb{Z}_{q}^{n \times k}, \mathbf{U}^{\prime} \leftarrow \operatorname{SamPre}\left(\mathbf{A}, \mathbf{V}^{\prime}\right.$, td $)$, we have the following statistical indistinguishability (negligible in $n$ )

$$
\mathbf{A} \stackrel{\text { stat }}{\approx} \mathbf{A}^{\prime} \text { and }(\mathbf{A}, \mathrm{td}, \mathbf{U}, \mathbf{V}) \stackrel{\text { stat }}{\approx}\left(\mathbf{A}, \mathrm{td}, \mathbf{U}^{\prime}, \mathbf{V}^{\prime}\right)
$$

and $\mathbf{U}^{\prime} \leftarrow \operatorname{SamPre}\left(\mathbf{A}, \mathbf{V}^{\prime}, \mathrm{td}\right)$ always satisfies $\mathbf{A} \mathbf{U}^{\prime}=\mathbf{V}^{\prime}$ and $\left\|\mathbf{U}^{\prime}\right\|_{\infty} \leq \beta_{\text {sam }}$.

3 Given $n, m, q$ as above, there is an efficiently and deterministically computable matrix $\mathbf{G} \in \mathbb{Z}_{q}^{n \times m}$ and a deterministic polynomial-time algorithm $\mathbf{G}^{-1}$ that on input $\mathbf{V} \in \mathbb{Z}_{q}^{n \times k}$ (for any integer $k$ ) outputs $\mathbf{R}=\mathbf{G}^{-1}(\mathbf{V})$ such that $\mathbf{R} \in\{0,1\}^{m \times k}$ and $\mathbf{G R}=\mathbf{V}$.

\subsubsection{Our Multi-Key Homomorphic Signature for Single Dataset}

In this section, we present our multi-key homomorphic signature that supports the evaluation of boolean circuits of bounded polynomial depth. Our construction is inspired by the (single-key) one of Gorbunov et al. [77], with the fundamental difference that in our case we enable computations over data signed using different secret keys. Moreover, our scheme is secure against Type 3 forgeries. We achieve this via a new technique which consists into adding to every signature a component that specifically protects against this type of forgeries. We prove the scheme to be weakly-secure under the SIS hardness assumption.

Parameters. Before describing the scheme, we discuss how to set the various parameters involved. Let $\lambda$ be the security parameter, and let $d=d(\lambda)=$ poly $(\lambda)$ be the bound on the depth of the circuits supported by our scheme. We define the set of parameters used in our scheme $\operatorname{Par}=\left\{n, m, q, \beta_{\mathrm{SIS}}, \beta_{\max }, \beta_{\text {init }}\right\}$ in terms of $\lambda, d$ and of the parameters required by the trapdoor algorithm in Lemma 21: $m^{*}, \beta_{\text {sam }}$, where $m^{*}=m^{*}(n, q):=O(n \log q)$ and $\beta_{\text {sam }}:=O(n \sqrt{\log q})$. More precisely, we set: $\beta_{\max }:=2^{\omega(\log \lambda) d} ; \beta_{\mathrm{SIS}}:=2^{\omega(\log \lambda)} \beta_{\max } ; n=\operatorname{poly}(\lambda) ; q=$ 
$\mathcal{O}\left(2^{\text {poly }(\lambda)}\right)>\beta_{\text {SIS }}$ is a prime (as small as possible) so that the $\operatorname{SIS}\left(n, m^{\prime}, q, \beta_{\mathrm{SIS}}\right)$ assumption holds for all $m^{\prime}=\operatorname{poly}(\lambda) ; m=\max \left\{m^{*}, n \log q+\omega(\log (\lambda))\right\}=$ $\operatorname{poly}(\lambda)$ and, finally, $\beta_{\text {init }}:=\beta_{\text {sam }}=\operatorname{poly}(\lambda)$.

Construction. The PPT algorithms (Setup, KeyGen, Sign, Eval, Ver) which define our construction of Multi-key Homomorphic Signatures work as follows:

Setup $\left(1^{\lambda}\right)$. The setup algorithm takes as input the security parameter $\lambda$ and generates the public parameters pp which include: the bound on the circuit depth $d$ (which defines the class $\mathbb{F}$ of functions supported by the scheme, i.e., boolean circuits of depth $d)$, the set $\operatorname{Par}=\left\{n, m, q, \beta_{\mathrm{SIS}}, \beta_{\max }, \beta_{\text {init }}\right\}$, the set $\mathcal{U}=\left\{\mathbf{U} \in \mathbb{Z}_{q}^{m \times m}:\|\mathbf{U}\|_{\infty} \leq \beta_{\max }\right\}$, the set $\mathcal{V}=\left\{\mathbf{V} \in \mathbb{Z}_{q}^{n \times m}\right\}$, descriptions of the message space $\mathcal{M}=\{0,1\}$, the tag space $\mathcal{T}=[\mathrm{T}]$, and the identity space ID $=[C]$, for integers $T, C \in \mathbb{N}$. In this construction, the tag space is of polynomial size, i.e., $\mathbf{T}=\operatorname{poly}(\lambda)$ while the identity space is essentially unbounded, i.e., we set $C=2^{\lambda}$. Also recall that $\mathcal{T}$ and ID immediately define the label space $\mathcal{L}=I D \times \mathcal{T}$. The final output is $\mathrm{pp}=\{d, \operatorname{Par}, \mathcal{U}, \mathcal{V}, \mathcal{M}, \mathcal{T}$, ID $\}$. We assume that these public parameters $\mathrm{pp}$ are input of all subsequent algorithms, and often omit them from the input explicitly.

KeyGen(pp). The key generation algorithm takes as input the public parameters pp and generates a key-triple (sk, ek, vk) defined as follows. First, it samples $\mathrm{T}$ random matrices $\mathbf{V}_{1}, \ldots, \mathbf{V}_{\mathrm{T}} \leftarrow \mathcal{V}$. Second, it runs $(\mathbf{A}, \mathrm{td}) \leftarrow$ $\operatorname{TrapGen}\left(1^{n}, 1^{m}, q\right)$ to generate a matrix $\mathbf{A} \in \mathbb{Z}_{q}^{n \times m}$ along with its trapdoor td. Then, it outputs sk $=\left(\mathrm{td}, \mathbf{A}, \mathbf{V}_{1}, \ldots, \mathbf{V}_{\mathrm{T}}\right)$, ek $=\mathbf{A}$, vk $=\left(\mathbf{A}, \mathbf{V}_{1}, \ldots, \mathbf{V}_{\mathrm{T}}\right)$. Note that it is possible to associate the key-triple to an identity id $\in$ ID, when we need to stress this explicitly we write $\left(\mathbf{s k}_{\mathrm{id}}, \mathrm{ek}_{\mathrm{id}}, \mathbf{v k _ { \text { id } }}\right)$. We also observe that the key generation process can be seen as the combination of two independent sub-algorithms $\left.\right|^{7} \mathrm{KeyGen}_{1}$ and $\mathrm{KeyGen}_{2}$, where $\left\{\mathbf{V}_{1}, \ldots \mathbf{V}_{\mathrm{T}}\right\} \leftarrow$ $\mathrm{KeyGen}_{1}(\mathrm{pp})$ and $(\mathbf{A}, \mathrm{td}) \leftarrow \mathrm{KeyGen}_{2}(\mathrm{pp})$.

Sign(sk, $\ell, \mathrm{m})$. The signing algorithm takes as input a secret key sk, a label $\ell=$ $(\mathrm{id}, \tau)$ for the message $\mathrm{m}$ and it outputs a signature $\sigma:=\left(\mathrm{m}, \mathrm{z}, \mathrm{I}, \mathbf{U}_{\text {id }}, \mathbf{Z}_{\text {id }}\right)$ where $\mathbf{I}=\{$ id $\}, \mathbf{U}_{\text {id }}$ is generated as $\mathbf{U}_{\text {id }} \leftarrow \operatorname{SamPre}\left(\mathbf{A}, \mathbf{V}_{\ell}-\mathrm{mG}\right.$, td) (using the algorithm SamPre from Lemma 21), $\mathbf{z}=\mathrm{m}$ and $\mathbf{Z}_{\text {id }}=\mathbf{U}_{\text {id }}$. The two latter terms are responsible for protection against Type 3 forgeries. Although they are redundant for fresh signatures, their value will become different from $\left(\mathrm{m}, \mathbf{U}_{\mathrm{id}}\right)$ during homomorphic operations, as we clarify later on. More generally, in our construction signatures are of the form $\sigma:=$ $\left(\mathrm{m}, \mathrm{z}, \mathrm{I},\left\{\mathbf{U}_{\text {id }}\right\}_{\text {id } \in \mathrm{I}},\left\{\mathbf{Z}_{\text {id }}\right\}_{\text {id } \in \mathrm{I}}\right)$ with $\mathrm{I} \subseteq \mathrm{ID}$ and $\mathbf{U}_{\text {id }}, \mathbf{Z}_{\text {id }} \in \mathcal{U}, \forall$ id $\in \mathrm{I}$.

\footnotetext{
${ }^{7}$ This splitting will be used to extend our multi-key homomorphic signature scheme from supporting a single dataset to support multiple datasets. This extension holds in the standard model and is described in Section 4.5.3.
} 
$\operatorname{Eval}\left(f,\left\{\left(\sigma_{i}, \mathrm{EKS}_{i}\right)\right\}_{i \in[t]}\right)$. The evaluation algorithm takes as input a $t$-input function $f: \mathcal{M}^{t} \longrightarrow \mathcal{M}$, and a set of pairs $\left\{\left(\sigma_{i}, \mathrm{EKS}_{i}\right)\right\}_{i \in[t]}$ where each $\sigma_{i}$ is a signature and each $\mathrm{EKS}_{i}$ is a set of evaluation keys. In our description below we treat $f$ as an arithmetic circuit over $\mathbb{Z}_{q}$ consisting of addition and multiplication gates 8 Therefore, we only describe how to evaluate homomorphically a fan-in-2 addition (resp. multiplication) gate as well as a unary multiplication-by-constant gate.

Let $g$ be a fan-in-2 gate with left input $\sigma_{\mathrm{L}}:=\left(\mathrm{m}_{\mathrm{L}}, \mathrm{z}_{\mathrm{L}}, \mathrm{I}_{\mathrm{L}}, \mathbf{U}_{\mathrm{L}}, \mathbf{Z}_{\mathrm{L}}\right)$ and right input $\sigma_{\mathrm{R}}:=\left(\mathrm{m}_{\mathrm{R}}, \mathrm{z}_{\mathrm{R}}, \mathrm{I}_{\mathrm{R}}, \mathbf{U}_{\mathrm{R}}, \mathbf{Z}_{\mathrm{R}}\right)$. To generate the signature $\sigma:=(\mathrm{m}, \mathrm{z}, \mathrm{l}, \mathbf{U}, \mathbf{Z})$ on the gate's output one proceeds as follows. First set $I=I_{L} \cup I_{R}$. Second, "expand" $\mathbf{U}_{\mathrm{L}}:=\left\{\mathbf{U}_{\mathrm{L}}^{\text {id }}\right\}_{\text {id } \in \mathrm{I}_{\mathrm{L}}}$ as:

$$
\hat{\mathbf{U}}_{\mathrm{L}}^{\text {id }}=\left\{\begin{array}{ll}
\mathbf{0} & \text { if id } \notin \mathrm{I}_{\mathrm{L}} \\
\mathbf{U}_{\mathrm{L}}^{\text {id }} & \text { if id } \in \mathrm{I}_{\mathrm{L}}
\end{array} \quad, \quad \forall \text { id } \in \mathrm{I} .\right.
$$

where $\mathbf{0}$ denotes an $(m \times m)$-matrix with all zero entries. Basically, we extend the set to be indexed over all identities in $I=I_{L} \cup I_{R}$ by inserting zero matrices for identities in $I \backslash I_{L}$. The analogous expansion process is applied to $\mathbf{U}_{\mathrm{R}}:=\left\{\mathbf{U}_{\mathrm{R}}^{\text {id }}\right\}_{\mathrm{id} \in \mathrm{I}_{\mathrm{L}}}, \mathbf{Z}_{\mathrm{L}}:=\left\{\mathbf{Z}_{\mathrm{L}}{ }^{\text {id }}\right\}_{\text {id } \in \mathrm{I}_{\mathrm{R}}}$ and $\mathbf{Z}_{\mathrm{R}}:=\left\{\mathbf{Z}_{\mathrm{R}}^{\text {id }}\right\}_{\text {id } \in \mathrm{I}_{\mathrm{R}}}$, denoting the expanded sets $\left\{\hat{\mathbf{U}}_{\mathrm{R}}^{\text {id }}\right\}_{\text {id } \in \mathrm{I}},\left\{\hat{\mathbf{Z}}_{\mathrm{L}}^{\text {id }}\right\}_{\text {id } \in \mathrm{I}}$ and $\left\{\hat{\mathbf{Z}}_{\mathrm{R}}^{\text {id }}\right\}_{\text {id } \in \mathrm{I}}$ respectively.

Next, depending on whether $\mathrm{g}$ is an addition or multiplication gate one proceeds as follows.

Addition gate. If $\mathrm{g}$ is additive, compute $\mathrm{m}=\mathrm{m}_{\mathrm{L}}+\mathrm{m}_{\mathrm{R}}, \mathrm{z}=\mathrm{z}_{\mathrm{L}}+\mathrm{z}_{\mathrm{R}}$, $\mathbf{U}=\left\{\mathbf{U}_{\text {id }}\right\}_{\text {id } \in \mathrm{I}}:=\left\{\hat{\mathbf{U}}_{\mathrm{L}}^{\text {id }}+\hat{\mathbf{U}}_{\mathrm{R}}^{\text {id }}\right\}_{\text {id } \in \mathrm{I}}$ and $\mathbf{Z}=\left\{\mathbf{Z}_{\text {id }}\right\}_{\text {id } \in \mathrm{I}}:=\left\{\hat{\mathbf{Z}}_{\mathrm{L}}^{\text {id }}+\hat{\mathbf{Z}}_{\mathrm{R}}^{\text {id }}\right\}_{\text {id } \in \mathbf{I}}$.

If we refer to $\beta_{\mathrm{L}}$ and $\beta_{\mathrm{R}}$ as $\left\|\mathbf{U}_{\mathrm{L}}\right\|_{\infty}:=\max \left\{\left\|\mathbf{U}_{\mathrm{L}}^{\mathrm{id}}\right\|_{\infty}:\right.$ id $\left.\in \mathrm{I}_{\mathrm{L}}\right\}$ and $\left\|\mathbf{U}_{\mathrm{R}}\right\|_{\infty}:=$ $\max \left\{\left\|\mathbf{U}_{\mathrm{R}}^{\text {id }}\right\|_{\infty}:\right.$ id $\left.\in \mathrm{I}_{\mathrm{R}}\right\}$ respectively, then for any fan-in-2 addition gate it holds $\beta:=\|\mathbf{U}\|_{\infty}=\beta_{\mathrm{L}}+\beta_{\mathrm{R}}$. The same noise growth applies to $\mathbf{Z}$.

Multiplication gate. If $\mathrm{g}$ is multiplicative, compute $\mathrm{m}=\mathrm{m}_{\mathrm{L}} \cdot \mathrm{m}_{\mathrm{R}}, \mathrm{z}=$ $\mathrm{z}_{\mathrm{L}}+\mathrm{z}_{\mathrm{R}}$, define $\mathbf{V}_{\mathrm{L}}=\sum_{\mathrm{id} \in \mathrm{I}_{\mathrm{L}}} \mathbf{A}_{\mathrm{id}} \mathbf{U}_{\text {id }}+\mathrm{m}_{\mathrm{L}} \mathbf{G}$, set

$$
\mathbf{U}=\left\{\mathbf{U}_{\mathrm{id}}\right\}_{\mathrm{id} \in \mathrm{I}}:=\left\{\mathrm{m}_{\mathrm{R}} \hat{\mathbf{U}}_{\mathrm{L}}^{\mathrm{id}}+\hat{\mathbf{U}}_{\mathrm{R}}^{\mathrm{id}} \cdot \mathbf{G}^{-1}\left(\mathbf{V}_{\mathrm{L}}\right)\right\}_{\mathrm{id} \in \mathrm{I}}
$$

and $\mathbf{Z}=\left\{\mathbf{Z}_{\text {id }}\right\}_{\text {id } \in \mathrm{I}}:=\left\{\hat{\mathbf{Z}}_{\mathrm{L}}^{\text {id }}+\hat{\mathbf{Z}}_{\mathrm{R}}^{\text {id }}\right\}_{\text {id } \in \text { I }}$.

Letting $\beta_{\mathrm{L}}$ and $\beta_{\mathrm{R}}$ as defined before, then for any fan-in-2 multiplication gate it holds $\beta:=\|\mathbf{U}\|_{\infty}=\left|\mathrm{m}_{\mathrm{R}}\right| \beta_{\mathrm{L}}+m \beta_{\mathrm{R}}$, while the noise growth of $\mathbf{Z}$ is the same as in the addition gate.

\footnotetext{
${ }^{8}$ We point out that considering $f$ as an arithmetic circuit over $\mathbb{Z}_{q}$ is enough to describe any boolean circuits consisting of NAND gates as $\operatorname{NAND}\left(\mathrm{m}_{1}, \mathrm{~m}_{2}\right)=1-\mathrm{m}_{1} \cdot \mathrm{m}_{2}$ holds for $\mathrm{m}_{1}, \mathrm{~m}_{2} \in\{0,1\}$.
} 
Multiplication by constant gate. Let $g$ be a unary gate representing a multiplication by a constant $a \in \mathbb{Z}_{q}$, and let its single input signature be $\sigma_{\mathrm{R}}:=\left(\mathrm{m}_{\mathrm{R}}, \mathrm{z}_{\mathrm{R}}, \mathrm{I}_{\mathrm{R}}, \mathbf{U}_{\mathrm{R}}, \mathbf{Z}_{\mathrm{R}}\right)$. The output $\sigma:=(\mathrm{m}, \mathrm{z}, \mathrm{I}, \mathbf{U}, \mathbf{Z})$ is obtained by setting $\mathrm{m}=a \cdot \mathrm{m}_{\mathrm{R}} \in \mathbb{Z}_{q}, \mathbf{z}=\mathrm{z}_{\mathrm{R}}, \mathrm{I}=\mathrm{I}_{\mathrm{R}}, \mathbf{Z}=\mathbf{Z}_{\mathrm{R}}$, and $\mathbf{U}=\left\{\mathbf{U}^{\text {id }}\right\}_{\text {id } \in \mathrm{I}}$ where, for all id $\in \mathrm{I}, \mathbf{U}^{\text {id }}=a \cdot \mathbf{U}_{\mathrm{R}}^{\text {id }}$ or, alternatively, $\mathbf{U}^{\text {id }}=\mathbf{U}_{\mathrm{R}}^{\text {id }} \cdot \mathbf{G}^{-1}(a \cdot \mathbf{G})$. In the first case, the noise parameter becomes $\beta:=\|\mathbf{U}\|_{\infty}=|a| \beta_{\mathrm{L}}$ (thus $a$ needs to be small), whereas in the second case it holds $\beta:=\|\mathbf{U}\|_{\infty} \leq m \beta_{\mathrm{L}}$, which is independent of $a$ 's size.

$\operatorname{Ver}\left(\mathcal{P},\left\{\mathrm{vk}_{\mathrm{id}}\right\}_{\mathrm{id} \in \mathcal{P}}, \mathrm{m}, \sigma\right)$. The verification algorithm takes as input a labeled program $\mathcal{P}=\left(f, \ell_{1}, \ldots, \ell_{n}\right)$, the set of the verification keys $\left\{v k_{\text {id }}\right\}_{\text {id } \in \mathcal{P}}$ of users involved in the program $\mathcal{P}$, a message $\mathrm{m}$ and a signature $\sigma=(\mathrm{m}, \mathrm{z}, \mathrm{I}, \mathbf{U}, \mathbf{Z})$. It then performs three main checks and outputs 0 if at least one check fails, otherwise it returns 1 .

Firstly, it checks if the list of identities declared in $\sigma$ corresponds to the ones in the labels of $\mathcal{P}: \quad \mathrm{I}=\{$ id $:$ id $\in \mathcal{P}\}$

Secondly, from the circuit $f$ (again seen as an arithmetic circuit) and the values $\left\{\mathbf{V}_{\ell_{1}}, \ldots, \mathbf{V}_{\ell_{t}}\right\}$ contained in the verification keys, it computes two values $\mathbf{V}^{*}$ and $\mathbf{V}^{+}$proceeding gate by gate as follows. Given as left and right input matrices $\mathbf{V}_{\mathrm{L}}^{*}, \mathbf{V}_{\mathrm{R}}^{*}$ (resp. $\mathbf{V}_{\mathrm{L}}^{+}, \mathbf{V}_{\mathrm{R}}^{+}$), at every addition gate one computes $\mathbf{V}^{*}=\mathbf{V}_{\mathrm{L}}^{*}+\mathbf{V}_{\mathrm{R}}^{*}\left(\right.$ resp. $\mathbf{V}^{+}=\mathbf{V}_{\mathrm{L}}^{+}+\mathbf{V}_{\mathrm{R}}^{+}$); at every multiplication gate one computes $\mathbf{V}^{*}=\mathbf{V}_{\mathrm{R}}^{*} \mathbf{G}^{-1} \mathbf{V}_{\mathrm{L}}^{*}$ (resp. $\mathbf{V}^{+}=\mathbf{V}_{\mathrm{L}}^{+}+\mathbf{V}_{\mathrm{R}}^{+}$). Every gate representing a multiplication by a constant $a \in \mathbb{Z}_{q}$, on input $\mathbf{V}_{\mathrm{R}}^{*}\left(\right.$ resp. $\left.\mathbf{V}_{\mathrm{R}}^{+}\right)$ outputs $\mathbf{V}^{*}=a \cdot \mathbf{V}_{\mathrm{R}}^{*}\left(\right.$ resp. $\mathbf{V}^{+}=\mathbf{V}_{\mathrm{R}}^{+}$). Note that the computation of $\mathbf{V}^{+}$ is essentially the computation of a linear function $\mathbf{V}^{+}=\sum_{i=1}^{t} \gamma_{i} \cdot \mathbf{V}_{\ell_{i}}$, for some coefficients $\gamma_{i}$ that depend on the structure of the circuit $f$.

Thirdly, the verification algorithm parses $\mathbf{U}=\left\{\mathbf{U}_{\mathrm{id}}\right\}_{\text {id } \in \mathbf{I}}$ and $\mathbf{Z}=\left\{\mathbf{Z}_{\text {id }}\right\}_{\text {id } \in \mathbf{I}}$ and checks:

$$
\begin{aligned}
\|\mathbf{U}\|_{\infty} \leq \beta_{\max } \quad \text { and } \quad\|\mathbf{Z}\|_{\infty} \leq \beta_{\max } \\
\sum_{\mathrm{id} \in \mathrm{I}} \mathbf{A}_{\mathrm{id}} \mathbf{U}_{\mathrm{id}}+\mathrm{m} \cdot \mathbf{G}=\mathbf{V}^{*} \\
\sum_{\mathrm{id} \in \mathrm{I}} \mathbf{A}_{\mathrm{id}} \mathbf{Z}_{\mathrm{id}}+\mathbf{z} \cdot \mathbf{G}=\mathbf{V}^{+}
\end{aligned}
$$

Finally, it is worth noting that the computation of the matrices $\mathbf{V}^{*}$ and $\mathbf{V}^{+}$can be precomputed (or performed offline), prior to seeing the actual signature $\sigma$. In the multiple dataset extension of Section 4.5.3 this precomputation becomes particularly beneficial as the same $\mathbf{V}^{*}, \mathbf{V}^{+}$can be re-used every time one wants to verify for the same labeled program $\mathcal{P}$ (i.e., one can verify faster, in an amortized sense, than that of running $f$ ). 
In the following paragraphs we analyse the correctness, succinctness and the security of the proposed construction.

Noise Growth and Succinctness. First we analyse the noise growth of the components $\mathbf{U}, \mathbf{Z}$ in the signatures of our MKHSig construction. In particular we need to show that when starting from "fresh" signatures, in which the noise is bounded by $\beta_{\text {init }}$, and we apply an admissible circuit, then one ends up with signatures in which the noise is within the allowable amount $\beta_{\max }$.

An analysis similar to the one of Gorbunov et al. [77] is applicable also to our construction whenever the admissible functions are boolean circuits of depth $d$ composed only of NAND gates.

Let us first consider the case of the $\mathbf{U}$ component of the signatures. At every NAND gate, if $\left\|\mathbf{U}_{\mathrm{L}}\right\|_{\infty},\left\|\mathbf{U}_{\mathrm{R}}\right\|_{\infty} \leq \beta$, the noise of the resulting $\mathbf{U}$ is at most $(m+1) \beta$. Therefore, if the circuit has depth $d$, the noise of the matrix $\mathbf{U}$ at the end of the evaluation is bounded by $\|\mathbf{U}\|_{\infty} \leq \beta_{\text {init }} \cdot(m+1)^{d} \leq 2^{O(\log \lambda) d} \leq \beta_{\max }$. For what regards the computation performed over the matrices $\mathbf{Z}$, we observe that we perform only additions (or identity functions) over them. This means that at every gate of any $f$, the noise in the $\mathbf{Z}$ component at most doubles. Given that we consider depth- $d$ circuits we have that $\|\mathbf{Z}\|_{\infty} \leq \beta_{\text {init }} \cdot 2^{d} \leq 2^{O(\log \lambda)+d} \leq$ $\beta_{\max }$. Finally, by inspection one can see that the size of every signature $\sigma$ on a computation's output involving $n$ users is at most $\left(1+2^{d}+n \lambda+2 n \beta_{\max }\right)$ that is $O(n \cdot p(\lambda))$ for some fixed polynomial $p(\cdot)$.

Authentication Correctness. Each fresh signature of a message $\mathrm{m}$ labeled by $\ell:=(\mathrm{id}, \tau)$ is of the form $\sigma:=(\mathrm{m}, \mathbf{z}=\mathrm{m}, \mathrm{I}=\{\mathrm{id}\}, \mathbf{U}, \mathbf{Z}=\mathbf{U})$. In this case, the labeled program used by Ver to verify $\sigma$ is the identity program on $\mathrm{m}$ with label $\ell=($ id, $\tau)$. We need to show that for $\mathcal{P}=\mathcal{I}_{\ell}$, and $\mathrm{m}$ the signature $\sigma$ passes the four verification checks (4.1), 4.2), 4.3) and (4.4). It is trivial to check that (4.1) holds, since $\mathbf{I}=\{\mathrm{id}\}=\left\{\mathrm{id} \in \mathcal{I}_{\ell}\right\}$. Moreover, (4.2) trivially follows from the fact that $\beta_{\text {init }} \leq \beta_{\max }$. For what concerns equation (4.3) we have:

$$
\sum_{\mathrm{id} \in \mathrm{I}} \mathbf{A}_{\mathrm{id}} \mathbf{U}_{\mathrm{id}}+\mathrm{m} \cdot \mathbf{G}=\mathbf{A}_{\mathrm{id}} \mathbf{U}_{\mathrm{id}}+\mathrm{m} \cdot \mathbf{G}=\mathbf{V}_{\ell}=\mathbf{V}^{*}
$$

where the first equality holds because $\mathrm{I}=\{\mathrm{id}\}$, the second holds by the construction of $\mathbf{U}_{\mathrm{id}}$ in the Sign algorithm and the last one holds by construction of the Ver algorithm. Note that, since here $\mathbf{z}=\mathrm{m}$ and $\mathbf{U}_{\mathrm{id}}=\mathbf{Z}$, then (4.4) is trivially satisfied.

Evaluation Correctness. We prove the correctness of the Eval algorithm for a generic fan-in-2 addition and multiplication gate g: by associativity this proves the correctness of the algorithm on any circuit. Let $m_{L}\left(\right.$ resp. $\left.m_{R}\right)$ denote the left (resp. right) input wire of the considered gate $g$, let $\sigma_{\mathrm{L}}:=\left(\mathrm{m}_{\mathrm{L}}, \mathrm{z}_{\mathrm{L}}, \mathrm{l}_{\mathrm{L}}, \mathbf{U}_{\mathrm{L}}, \mathbf{Z}_{\mathrm{L}}\right)$ (resp. $\left.\sigma_{R}\right)$ be a signature that verifies correctly for $m_{L}\left(\right.$ resp. $\left.m_{R}\right)$ and program $\mathcal{P}_{\mathrm{L}}\left(\right.$ resp. $\left.\mathcal{P}_{\mathrm{R}}\right)$. Let $\sigma=\left(\mathrm{m}, \mathrm{z}, \mathrm{I}=\mathrm{I}_{\mathrm{L}} \cup \mathrm{I}_{\mathrm{R}}, \mathbf{U}, \mathbf{Z}\right)$ be the signature obtained after a gate evaluation. 
It is immediate to see that the set I satisfies check (4.1). Moreover, from the noise growth analysis given above, it results that equation $(4.2)$ is satisfied by both $\mathbf{U}$ and $\mathbf{Z}$.

g Additive Gate: According to the definition of Eval, we have $\sigma=\left(\mathrm{m}=: \mathrm{m}_{\mathrm{L}}+\right.$ $\mathrm{m}_{\mathrm{R}}, \mathrm{I}=\mathrm{I}_{\mathrm{L}} \cup \mathrm{I}_{\mathrm{R}}, \mathbf{U}=\left\{\mathbf{U}_{\mathrm{id}}\right\}_{\mathrm{id} \in \mathrm{I}}:=\left\{\hat{\mathbf{U}}_{\mathrm{L}}^{\mathrm{id}}+\hat{\mathbf{U}}_{\mathrm{R}}^{\mathrm{id}}\right\}_{\mathrm{id} \in \mathrm{I}}, \mathrm{z}:=\mathrm{z}_{\mathrm{L}}+\mathrm{z}_{\mathrm{R}}, \mathbf{Z}=\left\{\mathbf{Z}_{\text {id }}\right\}_{\mathrm{id} \in \mathrm{I}}:=$ $\left.\left\{\hat{\mathbf{Z}}_{\mathrm{L}}^{\mathrm{id}}+\hat{\mathbf{Z}}_{\mathrm{R}}^{\mathrm{id}}\right\}_{\mathrm{id} \in \mathrm{I}}\right)$.

Below we focus on the proof for equation (4.3), and notice that the case of equation 4.4 is analogous.

$$
\begin{aligned}
\sum_{\text {id } \in \mathrm{I}} \mathbf{A}_{\text {id }} \mathbf{U}_{\text {id }}+\mathrm{m} \cdot \mathbf{G} & =\sum_{\text {id } \in I} \mathbf{A}_{\text {id }}\left(\hat{\mathbf{U}}_{\mathrm{L}}^{\text {id }}+\hat{\mathbf{U}}_{\mathrm{R}}^{\mathrm{id}}\right)+\left(\mathrm{m}_{\mathrm{L}}+\mathrm{m}_{\mathrm{R}}\right) \cdot \mathbf{G} \\
& =\left(\sum_{\mathrm{id} \in \mathrm{I}_{\mathrm{L}}} \mathbf{A}_{\mathrm{id}} \mathbf{U}_{\mathrm{L}}^{\mathrm{id}}+\mathrm{m}_{\mathrm{L}} \cdot \mathbf{G}\right)+\left(\sum_{\mathrm{id} \in \mathrm{I}_{\mathrm{R}}} \mathbf{A}_{\mathrm{id}} \mathbf{U}_{\mathrm{R}}^{\mathrm{id}}+\mathrm{m}_{\mathrm{R}} \cdot \mathbf{G}\right) \\
& =\mathbf{V}_{\mathrm{L}}+\mathbf{V}_{\mathrm{R}}=\mathbf{V}^{*}
\end{aligned}
$$

where the second equation holds because $\hat{\mathbf{U}}_{\mathrm{L}}^{\text {id }}=\overrightarrow{0}^{m \times m}$ for any id $\in \mathrm{I} \backslash \mathrm{I}_{\mathrm{L}}$ (resp. $\quad \hat{\mathbf{U}}_{\mathrm{R}}^{\mathrm{id}}=\overrightarrow{0}^{m \times m}$ for any id $\in \mathrm{I} \backslash \mathrm{I}_{\mathrm{R}}$ ), while the last equation follows from the assumption on the valid verification of $\sigma_{\mathrm{L}}$ (resp. $\sigma_{\mathrm{R}}$ ) and from the definition of the Ver algorithm (w.r.t. the computation over the $\mathbf{V}_{\ell}$ ).

g Multiplicative Gate: According with the definition of Eval, we have $\sigma=$ $\left(\mathrm{m}=: \mathrm{m}_{\mathrm{L}} \cdot \mathrm{m}_{\mathrm{R}}, \mathrm{I}=\mathrm{I}_{\mathrm{L}} \cup \mathrm{I}_{\mathrm{R}}, \mathbf{U}=\left\{\mathbf{U}_{\mathrm{id}}\right\}_{\mathrm{id} \in \mathrm{I}}:=\left\{\mathrm{m}_{\mathrm{R}} \hat{\mathbf{U}}_{\mathrm{L}}^{\mathrm{id}}+\hat{\mathbf{U}}_{\mathrm{R}}^{\mathrm{id}} \cdot \mathrm{G}^{-1}\left(\mathbf{V}_{\mathrm{L}}\right)\right\}_{\mathrm{id} \in \mathrm{I}}, \mathrm{z}:=\right.$ $\left.\mathrm{z}_{\mathrm{L}}+\mathrm{z}_{\mathrm{R}}, \mathbf{Z}=\left\{\mathbf{Z}_{\text {id }}\right\}_{\text {id } \in \mathrm{I}}:=\left\{\hat{\mathbf{Z}}_{\mathrm{L}}^{\text {id }}+\hat{\mathbf{Z}}_{\mathrm{R}}^{\text {id }}\right\}_{\text {id } \in \mathrm{I}}\right)$, where $\mathbf{V}_{\mathrm{L}}=\sum_{\mathrm{id} \in \mathrm{I}_{\mathrm{L}}} \mathbf{A}_{\text {id }} \mathbf{U}_{\text {id }}+\mathrm{m}_{\mathrm{L}} \mathbf{G}$.

We focus on proving the case of equation (4.3); as the case of equation (4.4) follows from the same analysis of the additive gate shown above.

$$
\begin{aligned}
& \sum_{\text {id } \in I} \mathbf{A}_{\text {id }} \mathbf{U}_{\text {id }}+\mathrm{m} \cdot \mathbf{G}=\sum_{\text {id } \in \mathrm{I}} \mathbf{A}_{\text {id }}\left(\mathrm{m}_{\mathrm{R}} \hat{\mathbf{U}}_{\mathrm{L}}^{\mathrm{id}}+\hat{\mathbf{U}}_{\mathrm{R}}^{\text {id }} \cdot \mathbf{G}^{-1}\left(\mathbf{V}_{\mathrm{L}}\right)\right)+\left(\mathrm{m}_{\mathrm{L}} \cdot \mathrm{m}_{\mathrm{R}}\right) \cdot \mathbf{G} \\
& =m_{R} \cdot\left(\sum_{i d \in I} \mathbf{A}_{\text {id }} \hat{\mathbf{U}}_{\mathrm{L}}^{\text {id }}+m_{L} \mathbf{G}\right)+\left(\sum_{\text {id } \in \mathrm{I}} \mathbf{A}_{\text {id }} \hat{\mathbf{U}}_{\mathrm{R}}^{\text {id }}\right) \mathbf{G}^{-1}\left(\mathbf{V}_{\mathrm{L}}\right) \\
& =\mathrm{m}_{\mathrm{R}} \mathbf{V}_{\mathrm{L}}+\left(\sum_{\mathrm{id} \in \mathrm{I}} \mathbf{A}_{\mathrm{id}} \hat{\mathbf{U}}_{\mathrm{R}}^{\mathrm{id}}\right) \mathbf{G}^{-1}\left(\mathbf{V}_{\mathrm{L}}\right) \\
& =\mathrm{m}_{\mathrm{R}} \mathrm{GG}^{-1}\left(\mathbf{V}_{\mathrm{L}}\right)+\left(\sum_{\mathrm{id} \in \mathrm{I}} \mathbf{A}_{\mathrm{id}} \hat{\mathbf{U}}_{\mathrm{R}}^{\text {id }}\right) \mathrm{G}^{-1}\left(\mathbf{V}_{\mathrm{L}}\right) \\
& =\left[\left(\sum_{i d \in I_{R}} \mathbf{A}_{i d} \mathbf{U}_{R}^{\text {id }}\right)+m_{R} G\right] G^{-1}\left(V_{L}\right)=V_{R} G^{-1}\left(V_{L}\right)=V^{*} \text {. }
\end{aligned}
$$


In the first two equations we expand $\mathbf{U}$ and rearrange the terms grouping by $\mathbf{G}^{-1}\left(\mathbf{V}_{\mathrm{L}}\right)$ and $\mathrm{m}_{\mathrm{R}}$ respectively. The third equation follows from the definition of $V_{\mathrm{L}}$, while in the fourth one, we just rewrite $\mathrm{V}_{\mathrm{L}}$ as $\mathbf{G G}^{-1}\left(\mathrm{~V}_{\mathrm{L}}\right)$ : this allows to group by $\mathbf{G}^{-1}\left(\mathbf{V}_{\mathrm{L}}\right)$ in the fifth equation. The last two equations respectively follow from the definition of $\mathbf{V}_{\mathbf{L}}$ and $\mathbf{V}^{*}$.

Security. The following theorem states the security of the scheme MKHSig.

Theorem 17. If the $\operatorname{SIS}\left(n, m \cdot Q_{\text {id }}, q, \beta_{\mathrm{SIS}}\right)$ hardness assumption holds, MKHSig $=$ (Setup, KeyGen, Sign, Eval, Ver) is a multi-key homomorphic signature weakly-adaptive secure against adversaries that make signing queries involving at most $Q_{\text {id }}$ different identities and that make non-adaptive corruption queries.

Proof. Note that we can deal with corruptions via our generic result of Proposition 2. Therefore it is sufficient to prove the security against adversaries that make no corruptions. Moreover, since this scheme works for a single dataset note that Type 1 forgeries cannot occur.

For the proof let us recall how the weakly-adaptive security experiment (Definition 20 works for our multi-key homomorphic signature scheme MKHSig. This is a game between an adversary $\mathcal{A}$ and a challenger $\mathcal{C}$ that has four main phases:

$1 \mathcal{A}$ declares an integer $Q$ representing the number of different identities that it will ask in the signing queries. Moreover, for every $i \in[Q] \mathcal{A}$ sends to $\mathcal{C}$ a set $\mathcal{T}_{i} \subseteq \mathcal{T}:=\left\{\tau_{1}, \ldots, \tau_{\mathrm{T}}\right\}$ and a set of pairs $\left\{\left(\mathrm{m}_{\tau}, \tau\right)\right\}_{\tau \in \mathcal{T}_{i}}$.

$2 \mathcal{C}$ runs $\operatorname{Setup}\left(1^{\lambda}\right)$ to obtain the public parameters and sends them to $\mathcal{A}$.

$3 \mathcal{A}$ adaptively queries identities $\mathrm{id}_{1}, \ldots, \mathrm{id}_{Q}$. When $\mathcal{C}$ receives the query $\mathrm{id}_{i}$ it generates a key-triple $\left(\mathrm{sk}_{\mathrm{id}_{i}}, \mathrm{ek}_{\mathrm{id}_{i}}, \mathrm{vk}_{\mathrm{id}_{i}}\right)$ by running KeyGen(pp), and for all labels $\ell=\left(\mathrm{id}_{i}, \tau\right)$ such that $\tau \in \mathcal{T}_{i}$ it runs $\sigma_{\tau}^{i} \leftarrow \operatorname{Sign}\left(\mathrm{sk}_{\mathrm{id}_{i}}, \ell, \mathrm{m}_{\tau}\right)$. Then $\mathcal{C}$ sends to $\mathcal{A}$ : the public keys $\mathbf{v k}_{\mathrm{id}_{i}}:=\left(\mathbf{A}_{\mathrm{id}_{i}},\left\{\mathbf{V}_{\ell}\right\}_{\tau \in \mathcal{T}}\right)$ and $\mathrm{ek}_{\mathrm{id}_{i}}:=\left(\mathbf{A}_{\mathrm{id}_{i}}\right)$, and the signatures $\left\{\sigma_{\tau}^{i}\right\}_{\tau \in \mathcal{T}_{i}}$.

4 The adversary produces a forgery consisting of a labeled program $\mathcal{P}^{*}=\left(f^{*}\right.$, $\left.\ell_{1}^{*}, \ldots, \ell_{t}^{*}\right)$ where $f^{*} \in \mathbb{F}, f^{*}: \mathcal{M}^{t} \rightarrow \mathcal{M}$, a message $\mathrm{m}^{*}$ and a signature $\sigma^{*}$.

$\mathcal{A}$ wins the non-adaptive security game if $\operatorname{Ver}\left(\mathcal{P}^{*},\left\{\mathrm{vk}_{\mathrm{id}}\right\}_{\mathrm{id} \in \mathcal{P}^{*}}, \mathrm{~m}^{*}, \sigma^{*}\right)=1$ and one of the following conditions holds:

Type 2 Forgery: there exist messages $\mathrm{m}_{\ell_{1}^{*}}, \ldots, \mathrm{m}_{\ell_{t}^{*}}$ s.t. $\mathrm{m}^{*} \neq f^{*}\left(\mathrm{~m}_{\ell_{1}^{*}}, \ldots, \mathrm{m}_{\ell_{t}^{*}}\right)$ (i.e., $\mathrm{m}^{*}$ is not the correct output of $\mathcal{P}^{*}$ when executed over previously signed messages).

Type 3 Forgery: there exists at least one label $\ell^{*}=\left(\mathrm{id}^{*}, \tau^{*}\right)$ that was not queried by $\mathcal{A}$. 
Consider a variation of the above game obtained modifying phase 4.5 .2 as follows:

4.5.2) $\mathcal{C}$ picks an instance $\mathbf{A} \in \mathbb{Z}_{q}^{n \times m^{\prime}}$ of the $\operatorname{SIS}\left(n, m^{\prime}, q, \beta_{\text {SIS }}\right)$ problem for $m^{\prime}=$ $m \cdot Q=\operatorname{poly}(\lambda)$, and parse $\mathbf{A}:=\left(\mathbf{A}_{\mathrm{id}_{1}}|\ldots| \mathbf{A}_{\mathrm{id}_{Q}}\right) \in \mathbb{Z}_{q}^{n \times m^{\prime}}$ as the concatenation of $Q$ different blocks of $n \times m$ matrices.

Next, when $\mathcal{C}$ receives the $i$-th query $\operatorname{id}_{i}$ from $\mathcal{A}$, it does the following:

- it samples a matrix $\mathbf{U}_{\mathrm{id}_{i}, \tau} \leftarrow \mathcal{U}$ such that $\left\|\mathbf{U}_{\mathrm{id}_{i}, \tau}\right\| \leq \beta_{\text {init }}$;

- for all $\ell:=\left(\mathrm{id}_{i}, \tau\right)$ with $\tau \in \mathcal{T}_{i}, \mathcal{C}$ computes $\mathbf{V}_{\ell}=\mathbf{A}_{\mathrm{id}_{i}} \mathbf{U}_{\mathrm{id}_{i}, \tau}+\mathrm{m}_{\tau} \cdot \mathbf{G}$;

- for all $\ell:=\left(\mathrm{id}_{i}, \tau\right)$ with $\tau \notin \mathcal{T}_{i}, \mathcal{C}$ computes $\mathbf{V}_{\ell}=\mathbf{A}_{\mathrm{id}_{i}} \mathbf{U}_{\mathrm{id}_{i}, \tau}+b_{i, \tau} \cdot \mathbf{G}$, where $b_{i, \tau} \stackrel{\leftrightarrow}{\leftarrow}\{0,1\}$.

- $\mathcal{C}$ sends to $\mathcal{A}$ the public keys $\mathbf{v k}_{\mathrm{id}_{i}}:=\left(\mathbf{A}_{\mathrm{id}_{i}},\left\{\mathbf{V}_{\ell}\right\}_{\tau \in \mathcal{T}}\right)$ and $\mathrm{ek}_{\mathrm{id}_{i}}:=\left(\mathbf{A}_{\mathrm{id}_{i}}\right)$, along with signatures $\left\{\sigma_{\tau}^{i}\right\}_{\tau \in \mathcal{T}_{i}}$ where $\sigma_{\tau}^{i}:=\left(\mathbf{m}_{\tau}, \mathrm{m}_{\tau}, \mathrm{l}:=\left\{\mathrm{id}_{i}\right\}, \mathbf{U}_{\mathrm{id}_{i}, \tau}, \mathbf{U}_{\mathrm{id}_{i}, \tau}\right)$.

Clearly, if $\mathbf{A}$ is a uniformly random matrix so is each block $\left\{\mathbf{A}_{\mathrm{id}_{i}}\right\}_{i \in[Q]}$.

Due to point (2) of Lemma 21 , since $\left(\mathbf{A}_{\mathrm{id}_{i}} \mathbf{U}_{\mathrm{id}_{i}, \tau}\right)$ is statistically indistinguishable from a random matrix, all the matrices $\mathbf{V}_{\ell}$ generated in (4.5.2) are statistically close to the ones generated in (4.5.2). Thus, the two games are statistically indistinguishable. At this point we show that for every PPT adversary $\mathcal{A}$ which produces a forgery in the modified game we can construct a PPT algorithm $\mathcal{B}$ that solves the $\operatorname{SIS}\left(n, m \cdot Q, q, \beta_{\mathrm{SIS}}\right)$ problem. $\mathcal{B}$ receives an SIS instance $\mathbf{A}:=$ $\left(\mathbf{A}_{\mathrm{id}_{1}}|\ldots| \mathbf{A}_{\mathrm{id}_{Q}}\right) \in \mathbb{Z}_{q}^{n \times m Q}$ and simulates the modified game to $\mathcal{A}$ by acting exactly as the challenger $\mathcal{C}$ described above. Then, once $\mathcal{A}$ outputs its forgery, according to the forgery's type, $\mathcal{B}$ proceeds as described below.

Type 2 Forgeries. Let $\left(\mathcal{P}^{*}:=\left(f^{*}, \ell_{1}^{*}, \ldots, \ell_{t}^{*}\right), \mathrm{m}^{*}, \sigma^{*}:=\left(\mathrm{m}^{*}, \mathbf{z}^{*}, \mathbf{I}^{*}, \mathbf{U}^{*}, \mathbf{Z}^{*}\right)\right)$ be a Type 2 forgery produced by $\mathcal{A}$ in the modified game. Moreover let $\sigma=$ $(\mathrm{m}, \mathrm{z}, \mathrm{I}, \mathbf{U}, \mathbf{Z})$ be the signature obtained by honestly applying Eval to the signatures corresponding to labels $\ell_{1}^{*}, \ldots, \ell_{t}^{*}$ that were given to $\mathcal{A}$. Parse $\mathbf{U}:=$ $\left\{\mathbf{U}_{\text {id }}\right\}_{\text {id } \in \text { I }}$ and notice that by the correctness of the scheme we have that $\mathrm{m}=$ $f^{*}\left(\mathrm{~m}_{\ell_{1}^{*}}, \ldots, \mathrm{m}_{\ell_{t}^{*}}\right), \mathrm{I}=\left\{\right.$ id $:$ id $\left.\in \mathcal{P}^{*}\right\}$, and

$$
\sum_{\text {id } \in \mathrm{I}} \mathbf{A}_{\mathrm{id}} \mathbf{U}_{\mathrm{id}}+\mathrm{m} \cdot \mathbf{G}=\mathbf{V}^{*}
$$

Moreover, by definition of Type 2 forgery recall that $\mathrm{m}^{*} \neq f^{*}\left(\mathrm{~m}_{\ell_{1}^{*}}, \ldots, \mathrm{m}_{\ell_{t}^{*}}\right)$ and that the tuple satisfies verification. In particular, satisfaction of check 4.1) implies that $\mathrm{I}=\mathrm{I}^{*}$, while check 4.3 means

$$
\sum_{\mathrm{id} \in \mathrm{I}^{*}} \mathbf{A}_{\mathrm{id}} \mathbf{U}_{\mathrm{id}}^{*}+\mathrm{m}^{*} \cdot \mathbf{G}=\mathbf{V}^{*}
$$


. Combining the two equations above we obtain $\sum_{\text {id } \in \text { I }} \mathbf{A}_{\text {id }} \tilde{\mathbf{U}}_{\text {id }}=\tilde{\mathrm{m}} \cdot \mathbf{G}$, where $\tilde{\mathrm{m}}=\mathrm{m}-\mathrm{m}^{*} \neq \mathbf{0}$ and, for all id $\in \mathrm{I}, \tilde{\mathbf{U}}_{\text {id }}=\mathbf{U}_{\text {id }}^{*}-\mathbf{U}_{\text {id }} \in \mathcal{U}$ such that $\left\|\tilde{\mathbf{U}}_{\text {id }}\right\|_{\infty} \leq \beta_{\max }$. Notice that there must exist at least one $\overline{\mathrm{id}} \in \mathrm{I}$ for which $\tilde{\mathbf{U}}_{\overline{\mathrm{id}}} \neq \mathbf{0}$.

Moreover, for all id $\in\left\{\operatorname{id}_{1}, \ldots, \mathrm{id}_{Q}\right\} \backslash \mathrm{I}$, define $\tilde{\mathbf{U}}_{\mathrm{id}}=\mathbf{0}$ and set $\tilde{\mathbf{U}}=\left(\begin{array}{c}\tilde{\mathrm{U}}_{\mathrm{id}_{1}} \\ \vdots \\ \tilde{\mathrm{U}}_{\mathrm{id}_{Q}}\end{array}\right) \in$ $\mathbb{Z}_{q}^{m Q \times m}$. Then, we have $\mathbf{A} \tilde{\mathbf{U}}=\tilde{\mathrm{m}} \cdot \mathbf{G}$.

Next $\mathcal{B}$ samples $\mathbf{r} \leftrightarrow\{0,1\}^{m Q}$, sets $\mathbf{s}=\mathbf{A r} \in \mathbb{Z}_{q}^{n}$, and computes $\mathbf{r}^{\prime}=\mathbf{G}^{-1}\left(\tilde{\mathbf{m}}^{-1}\right.$. $\mathbf{s})$, so that $\mathbf{r}^{\prime} \in\{0,1\}^{m}$ and $\tilde{\mathbf{m}} \cdot \mathbf{G} \mathbf{r}^{\prime}=\mathbf{s}$. Finally, $\mathcal{B}$ outputs $\mathbf{u}=\tilde{\mathbf{U}} \mathbf{r}^{\prime}-\mathbf{r} \in \mathbb{Z}_{q}^{m Q}$. We conclude the proof by claiming that the vector $\mathbf{u}$ returned by $\mathcal{B}$ is a solution of the SIS problem for the matrix A. To see this observe that

$$
\mathbf{A}\left(\tilde{\mathbf{U}} \mathbf{r}^{\prime}-\mathbf{r}\right)=(\mathbf{A} \tilde{\mathbf{U}}) \mathbf{r}^{\prime}-\mathbf{A r}=\tilde{\mathbf{m}} \cdot \mathbf{G} \cdot \mathbf{G}^{-1}\left(\tilde{\mathrm{m}}^{-1} \cdot \mathbf{s}\right)-\mathbf{s}=\mathbf{0} .
$$

and $\|\mathbf{u}\|_{\infty} \leq(2 m+1) \beta_{\max } \leq \beta_{\text {SIS }}$.

It remains to show that $\mathbf{u} \neq \mathbf{0}$. We show that this is the case (i.e., $\tilde{\mathbf{U}} \mathbf{r}^{\prime} \neq \mathbf{r}$ ) with overwhelming probability by using an entropy argument (the same argument used in [77]). In particular, this holds for any (worst case) choice of $\mathbf{A}, \tilde{\mathbf{U}}, \tilde{\mathbf{m}}$, and only based on the random choice of $\mathbf{r} \leftarrow^{\$}\{0,1\}^{m Q_{\text {id }}}$. The intuition is that, even if $\mathbf{r}^{\prime}=\mathbf{G}^{-1}\left(\mathbf{s} \hat{m}^{-1}\right)$ depends on $\mathbf{s}=\mathbf{A r}, \mathbf{s}$ is too small to reveal much information about the random $\mathbf{r}$. More precisely, we have that $\mathbf{H}_{\infty}\left(\mathbf{r} \mid \mathbf{r}^{\prime}\right) \geq \mathbf{H}_{\infty}(\mathbf{r} \mid \mathbf{A r})$ because $\mathbf{r}^{\prime}$ is chosen deterministically based on $\mathbf{s}=\mathbf{A r}$. Due to the Lemma 20, we have that $\mathbf{H}_{\infty}(\mathbf{r} \mid \mathbf{A r}) \geq \mathbf{H}_{\infty}(\mathbf{r})-\log (|\mathcal{S}|)$, where $\mathcal{S}$ is the space of all possible $\mathbf{s}$. Since $\mathbf{s} \in \mathbb{Z}_{q}^{n},|\mathcal{S}|=q^{n}$, and then $\log (|\mathcal{S}|)=\log \left(q^{n}\right)=\log \left(\left(2^{\log q}\right)^{n}\right)=$ $n \log \left(\left(2^{\log q}\right)\right)=n \log q$. Regarding $\mathbf{H}_{\infty}(\mathbf{r})$, since $\mathbf{H}_{\infty}(X):=-\log \left(\max _{x} \operatorname{Pr}[X=\right.$ $x]$ ), we have $\mathbf{H}_{\infty}(\mathbf{r})=-\log \left(2^{-m Q}\right)=m Q \geq m$. Then,

$$
\mathbf{H}_{\infty}\left(\mathbf{r} \mid \mathbf{r}^{\prime}\right) \geq \mathbf{H}_{\infty}(\mathbf{r})-\log (\mathcal{S}) \geq m-n \log q=\omega(\log \lambda) .
$$

Since we know that for random variables $X, Y$ the optimal probability of an unbounded adversary guessing $X$ given the correlated value $Y$ is $2^{-\mathbf{H}_{\infty}(X \mid Y)}$, then $\operatorname{Pr}\left[\mathbf{r}=\tilde{\mathbf{U}} \mathbf{r}^{\prime}\right] \leq 2^{-\mathbf{H}_{\infty}\left(\mathbf{r} \mid \mathbf{r}^{\prime}\right)} \leq 2^{-\omega(\log \lambda)}=\operatorname{negl}(\lambda)$.

Type 3 Forgery. Let $\left(\mathcal{P}^{*}:=\left(f^{*}, \ell_{1}^{*}, \ldots, \ell_{t}^{*}\right), \mathrm{m}^{*}, \sigma^{*}:=\left(\mathrm{m}^{*}, \mathbf{z}^{*}, \mathbf{I}^{*}, \mathbf{U}^{*}, \mathbf{Z}^{*}\right)\right)$ be a Type 3 forgery produced by $\mathcal{A}$ in the modified game such that there exists (at least) one label $\ell_{j}^{*}=\left(\mathrm{id}^{*}, \tau^{*}\right)$ such that $\mathrm{id}^{*}=\mathrm{id}_{i}$ but $\tau^{*} \notin \mathcal{T}_{i}{ }^{9}$ Actually, without loss of generality we can assume that there is exactly one of such labels; if this is not the case, one could indeed redefine another adversary that makes more queries until it misses only this one. Note that for such a tag $\tau^{*} \notin \mathcal{T}_{i}, \mathcal{B}$ simulated $\mathbf{V}_{\mathrm{id}_{i}, \tau^{*}}=\mathbf{A U}_{\mathrm{id}_{i}, \tau^{*}}+b_{i, \tau^{*}} \mathbf{G}$ for a randomly chosen bit $b_{i, \tau^{*}} \stackrel{\$}{\$}\{0,1\}$, that is perfectly hidden from $\mathcal{A}$.

\footnotetext{
${ }^{9}$ It is easy to see that the case in which id* is new would imply the generation of a new $\mathbf{A}_{\text {id }^{*}}$, which would make the verification equations hold with negligible probability (over the random choice of $\left.\mathbf{A}_{\mathrm{id}^{*}}\right)$.
} 
By definition of Type 3 forgery, the tuple passes verification, and in particular check 4.4

$$
\sum_{\mathrm{id} \in I^{*}} \mathbf{A}_{\mathrm{id}} \mathbf{Z}_{\mathrm{id}}^{*}+\mathrm{z}^{*} \cdot \mathbf{G}=\mathbf{V}^{+}=\sum_{i=1}^{t} \gamma_{i} \cdot \mathbf{V}_{\ell_{i}^{*}}
$$

where the right hand side of the equation holds by construction of the verification algorithm.

Moreover, let $\sigma=(\mathrm{m}, \mathrm{z}, \mathrm{I}, \mathbf{U}, \mathbf{Z})$ be the signature obtained by honestly applying Eval to the signatures corresponding to labels $\ell_{1}^{*}, \ldots, \ell_{t}^{*}$; in particular for the specific, missing, label $\ell_{j}^{*} \mathcal{B}$ uses the values $\mathbf{U}_{\mathrm{id}_{i}, \tau^{*}}, b_{i, \tau^{*}}$ used to simulate $\mathbf{V}_{\mathrm{id}_{i}, \tau^{*}}$. Parsing $\mathbf{Z}:=\left\{\mathbf{Z}_{\text {id }}\right\}_{\text {id } \in \mathrm{I}}$, notice that by correctness it holds $\mathbf{I}=\left\{\right.$ id $:$ id $\left.\in \mathcal{P}^{*}\right\}$ and

$$
\sum_{\text {id } \in l} \mathbf{A}_{\text {id }} \mathbf{Z}_{\text {id }}+\mathbf{z} \cdot \mathbf{G}=\mathbf{V}^{+}
$$

where $\mathbf{z}=\sum_{i=1, i \neq j}^{t} \gamma_{i} \mathrm{~m}_{i}+\gamma_{j} b_{i, \tau^{*}}$. Now, the observation is that every $\gamma_{i} \leq 2^{d}<q$, i.e., $\gamma_{i} \neq 0 \bmod q$. Since $b_{i, \tau^{*}}$ is random and perfectly hidden to $\mathcal{A}$ we have that with probability $1 / 2$ it holds $\mathbf{z} \neq \mathbf{z}^{*}$.

Thus, if $\mathbf{z} \neq \mathbf{z}^{*}, \mathcal{B}$ combines the equalities on $\mathbf{V}^{+}$to come up with an equation

$$
\sum_{\text {id } \in \mathrm{I}} \mathbf{A}_{\text {id }} \tilde{\mathbf{Z}}_{\text {id }}=\tilde{\mathbf{z}} \cdot \mathbf{G}
$$

where $\tilde{\mathbf{z}}=\mathbf{z}-\mathbf{z}^{*} \neq 0 \bmod q$ and, for all id $\in \mathrm{I}, \tilde{\mathbf{Z}}_{\text {id }}=\mathbf{Z}_{\text {id }}^{*}-\mathbf{Z}_{\text {id }} \in \mathcal{U}$ such that $\left\|\tilde{\mathbf{Z}}_{\text {id }}\right\|_{\infty} \leq \beta_{\max }$.

Finally, using the same technique as in the case of Type 2 forgeries, $\mathcal{B}$ can compute a vector $\mathbf{u}$ that is a solution of SIS with overwhelming probability, i.e., $\mathrm{Au}=0$.

Therefore, we have proven that if an adversary $\mathcal{A}$ can break the MKHSig scheme with non negligible probability, then $\mathcal{C}$ can use such an $\mathcal{A}$ to break the SIS assumption for $\mathbf{A}$ with non negligible probability as well.

A Variant with Unbounded Tag Space in the Random Oracle Model. In this section, we show that the construction of multi-key homomorphic signatures of Section 4.5 .2 can be easily modified in order to have short public keys and to support an unbounded tag space $\mathcal{T}=\{0,1\}^{*}$. Note that once arbitrary tags are allowed, the scheme also allows to handle multiple datasets for free. In fact, one can always extend tags to include the dataset name, i.e., simply redefine each tag $\tau$ as consisting of two substrings $\tau=\left(\Delta, \tau^{\prime}\right)$ where $\Delta$ is the dataset name and $\tau^{\prime}$ the actual tag.

The idea of modifying the scheme to support an unbounded tag space is simple and was also suggested in [77] for their construction. Instead of sampling matrices $\left\{\mathbf{V}_{\text {id }, 1}, \ldots \mathbf{V}_{\text {id }, T}\right\}$ in KeyGen, one can just choose a random string $r_{\text {id }} \stackrel{\$}{\leftarrow}\{0,1\}^{\lambda}$ 
and define every $\mathbf{V}_{\mathrm{id}, \tau}:=\hat{\mathrm{H}}\left(r_{\mathrm{id}}, \tau\right)$ where $\hat{\mathrm{H}}:\{0,1\}^{*} \rightarrow \mathcal{V}$ is a hash function chosen in Setup (modeled as a random oracle in the proof). In all the remaining algorithms, every time one needs $\mathbf{V}_{\mathrm{id}, \tau}$, this is obtained using $\hat{\mathrm{H}}$.

For this modified scheme, we also provide an idea of how the security proof of Theorem 17 has to be modified to account for these changes. The main change is the simulation of hash queries, which is done as follows.

Before phase (4.5.2), where $\mathcal{A}$ declares its queries, $\mathcal{B}$ simply answers every query $\hat{\mathrm{H}}(r, \tau)$ with a randomly chosen $\mathrm{V} \stackrel{\$}{\leftarrow} \mathcal{V}$. Afterwards, once $\mathcal{A}$ has declared all its queries, $\mathcal{B}$ chooses $r_{\mathrm{id}_{1}}, \ldots, r_{\mathrm{id}_{Q}} \stackrel{\$}{\leftarrow}\{0,1\}^{\lambda}$ and programs the random oracle so that, for all $\tau \in \mathcal{T}_{i}, \hat{\mathrm{H}}\left(r_{\mathrm{id}_{i}}, \tau\right)=\mathbf{V}_{\mathrm{id}_{i}, \tau}$ where $\mathbf{V}_{\mathrm{id}_{i}, \tau}$ is the same matrix generated in the phase 4.5.2) of the modified game. On the other hand, for all $\tau \notin \mathcal{T}_{i}$, $\hat{\mathbf{H}}\left(r_{\mathrm{id}_{i}}, \tau\right)=\mathbf{V}_{\mathbf{i d}_{i}, \tau}$ where $\mathbf{V}_{\mathrm{id}_{i}, \tau}=\mathbf{A}_{\mathrm{id}_{\mathrm{d}}} \mathbf{U}_{\mathrm{id}_{i}, \tau}+b_{i, \tau} \mathbf{G}$ for a randomly chosen $\mathbf{U}_{\mathbf{i d}_{i}, \tau} \stackrel{\$}{\leftarrow}$ $\mathcal{U}$. All other queries $\hat{\mathrm{H}}(r, \tau)$ where $r \neq r_{\mathrm{id}_{i}}, \forall i \in[Q]$ are answered with random $\mathbf{V} \stackrel{\$}{\leftarrow} \mathcal{V}$. With this simulation, it is not hard to see that, from $\mathcal{A}$ 's forgery $\mathcal{B}$ can extract a solution for SIS (except for some negligible probability that $\mathcal{A}$ guesses one of $r_{\mathrm{id}_{i}}$ before seeing it).

\subsubsection{From a Single Dataset to Multiple Datasets}

In this section, we present a generic transformation to convert a single-dataset MKHSig scheme into a scheme that supports multiple datasets. The intuition behind this transformation is similar to the one employed in [77] and implicitly used in [49, 46], except that here we have to use additional techniques to deal with the multi-key setting. We combine a standard signature scheme NH.Sig (nonhomomorphic) with a single dataset multi-key homomorphic signature scheme MKHSig'. The idea is that for every new dataset $\Delta$, every user generates fresh keys of the multi-key homomorphic scheme MKHSig' and then uses the standard signature scheme NH.Sig to sign the dataset identifier $\Delta$ together with the generated public key. More precisely, in our transformation we assume to start with (single-dataset) multi-key homomorphic signature schemes in which the key generation algorithm can be split into two independent algorithms: KeyGen Kat $_{1}$ outputs some public parameters related to the identity id, and KeyGen ${ }_{2}$ which outputs the actual keys. Differently than [77], in our scheme the signer does not need to sign the whole dataset at once, nor has to fix a bound $N$ on the dataset size (unless such a bound is already contained in MKHSig').

In more details, let NH.Sig $=($ NH.KeyGen, NH.Sign, NH.Ver $)$ be a standard (non-homomorphic) signature scheme, and let MKHSig' $=\left(\right.$ Setup ${ }^{\prime}, K_{\text {KeyGen' }}$, Sign', $^{\prime}$

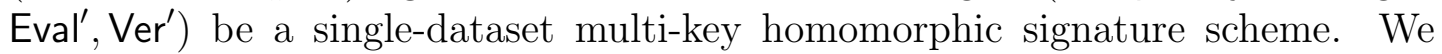
construct a multi-dataset multi-key homomorphic signature scheme MKHSig = (Setup, KeyGen, Sign, Eval, Ver) as follows.

Setup $\left(1^{\lambda}\right)$. The setup algorithm samples parameters of the single-dataset multi- 
key homomorphic signature scheme, $\mathrm{pp}^{\prime} \leftarrow \operatorname{Setup}^{\prime}\left(1^{\lambda}\right)$, together with a description of a PRF $F: K \times\{0,1\}^{*} \rightarrow\{0,1\}^{\rho}$, and outputs pp $=\left(\mathrm{pp}^{\prime}, F\right)$.

KeyGen(pp). The key generation algorithm runs NH.KeyGen to get $\left(\mathrm{pk}_{\mathrm{id}}^{\mathrm{NH}}, \mathrm{sk}_{\mathrm{id}}^{\mathrm{NH}}\right), \mathrm{a}$ pair of keys for the standard signature scheme. In addition, it runs KeyGen ${ }_{1}$ to generate user-specific public parameters $\mathrm{pp}_{\text {id }}$, and chooses a seed $\mathrm{K}_{\text {id }}$ for the PRF F. The final output is the vector $\left(s k_{i d}, e k_{i d}, v k_{i d}\right):$ where $s k_{i d}=$ $\left(\mathrm{sk}_{\mathrm{id}}^{\mathrm{NH}}, \mathrm{K}_{\mathrm{id}}\right), \mathrm{ek}_{\mathrm{id}}=\left(\mathrm{pp}_{\mathrm{id}}\right)$ and $\mathrm{vk}_{\mathrm{id}}=\left(\mathrm{pk}_{\mathrm{id}}^{\mathrm{NH}}, \mathrm{pp}_{\mathrm{id}}\right)$.

$\operatorname{Sign}\left(\mathrm{sk}_{\mathrm{id}}, \Delta, \ell, \mathrm{m}\right)$. The signing algorithm proceeds as follows. First it samples the keys of the single-dataset multi-key homomorphic signature scheme by feeding randomness $F_{\mathrm{K}_{\text {id }}}(\Delta)$ to $\mathrm{KeyGen}_{2}$, i.e., it runs $\mathrm{KeyGen}_{2}\left(\mathrm{pp} ; F_{\mathrm{K}_{\text {id }}}(\Delta)\right)$ to obtain the keys $\left(\mathbf{s k}_{\mathrm{id}}^{\Delta}, \mathrm{ek}_{\mathrm{id}}^{\Delta}, \mathrm{vk}_{\mathrm{id}}^{\Delta}\right){ }^{10}$ The algorithm then runs $\sigma^{\prime} \leftarrow \operatorname{Sign}^{\prime}\left(\mathbf{s k}_{\mathrm{id}}^{\Delta}\right.$, $\ell, \mathrm{m})$, and uses the non-homomorphic scheme to sign the concatenation of the public key $\mathrm{vk}_{\mathrm{id}}^{\Delta}$ and the dataset identifier $\Delta$, i.e., $\sigma_{\mathrm{id}}^{\Delta} \leftarrow \mathrm{NH} \cdot \operatorname{Sign}\left(\mathrm{sk}_{\mathrm{id}}^{\mathrm{NH}}, \mathrm{vk}_{\mathrm{id}}^{\Delta}\right.$ $\mid \Delta)$, The output is the tuple $\sigma:=\left(\mathrm{I}=\{\mathrm{id}\}, \sigma^{\prime}, \operatorname{par}_{\Delta}\right)$ where $\operatorname{par}_{\Delta}=\left\{\left(\mathrm{ek}_{\mathrm{id}}^{\Delta}, \mathrm{vk}_{\mathrm{id}}^{\Delta}, \sigma_{\text {id }}^{\Delta}\right)\right\}$. Note that the use of the PRF allows every signer (having the same $\mathrm{K}_{\mathrm{id}}$ ) to generate the same keys of the scheme MKHSig' on the same dataset $\Delta$.

$\operatorname{Eval}\left(f,\left\{\left(\sigma_{i}, \mathrm{EKS}_{i}\right)\right\}_{i \in[t]}\right)$. For each $i \in[t]$, the algorithm parses every signature as $\sigma_{i}:=\left(\mathbf{I}_{i}, \sigma_{i}^{\prime}, \operatorname{par}_{\Delta, i}\right)$ with $\operatorname{par}_{\Delta, i}=\left\{\mathrm{ek}_{\mathrm{id}}^{\Delta}, \mathrm{vk}_{\mathrm{id}}^{\Delta}, \sigma_{\mathrm{id}}^{\Delta}\right\}_{\mathrm{id} \in \mathrm{I}_{i}}$, and sets $\mathrm{EKS}_{i}^{\prime}=$ $\left\{\text { ek }_{\text {id }}^{\Delta}\right\}_{\text {id } \in I_{i}}$. It computes $\sigma^{\prime} \leftarrow \operatorname{Eval}^{\prime}\left(f,\left\{\sigma_{i}^{\prime}, \mathrm{EKS}_{i}^{\prime}\right\}_{i \in[t]}\right)$, defines $\mathbf{I}=\cup_{i=1}^{t} I_{i}$ and $\operatorname{par}_{\Delta}=\cup_{i=1}^{t} \operatorname{par}_{\Delta, i}$. The final output is $\sigma=\left(\mathrm{I}, \sigma^{\prime}, \operatorname{par}_{\Delta}\right)$.

$\operatorname{Ver}\left(\mathcal{P}, \Delta,\left\{\mathrm{vk}_{\mathrm{id}}\right\}_{\mathrm{id} \in \mathcal{P}}, \mathrm{m}, \sigma\right)$. The verification algorithm begins by parsing the verification keys as $v k_{i d}:=\left(\mathrm{pk}_{\mathrm{id}}^{\mathrm{NH}}, \mathrm{pp}_{\mathrm{id}}\right)$ for each id $\in \mathrm{I}$, and also the signature as $\sigma=\left(\mathrm{I}, \sigma^{\prime}, \operatorname{par}_{\Delta}\right)$ with $\operatorname{par}_{\Delta}=\left\{\left(\mathrm{ek}_{\mathrm{id}}^{\Delta}, \mathrm{vk}_{\mathrm{id}}^{\Delta}, \sigma_{\text {id }}^{\Delta}\right)\right\}_{\mathrm{id} \in \mathrm{I}}$. Then, it proceeds with two main steps. First, for each id $\in \mathrm{I}$, it verifies the standard signature $\sigma_{\mathrm{id}}^{\Delta}$ on the public key of the single-dataset multi-key homomorphic scheme and the given dataset, i.e., it checks whether $\mathrm{NH} . \operatorname{Ver}\left(\mathrm{pk}_{\mathrm{id}}^{\mathrm{NH}}, \mathrm{vk}_{\mathrm{id}}^{\Delta} \mid \Delta\right.$, $\left.\sigma_{\text {id }}^{\Delta}\right)=1, \forall$ id $\in$ I. If at least one of the previous equations is not satisfied, the algorithm returns 0 , otherwise it proceeds to the second check and returns the output of $\operatorname{Ver}^{\prime}\left(\mathcal{P},\left\{\mathrm{pp}_{\mathrm{id}}, \mathrm{vk}_{\mathrm{id}}^{\Delta}\right\}_{\mathrm{id} \in \mathcal{P}}, \mathrm{m}, \sigma^{\prime}\right)$.

Authentication Correctness. Correctness of the scheme substantially follows from the correctness of the regular signature scheme NH.Sig, the singledataset multi-hey homomorphic scheme MKHSig' and the PRF F. More formally, a fresh signature $\sigma$ on $\left(\mathrm{sk}_{\mathrm{id}}^{\Delta}, \Delta, \ell, \mathrm{m}\right)$ is of the form $\left(\mathrm{I}, \sigma^{\prime}, \operatorname{par}_{\Delta}\right)$ where $\operatorname{par}_{\Delta}=\left\{\mathrm{ek}_{\mathrm{id}}^{\Delta}, \mathrm{vk}_{\mathrm{id}}^{\Delta}, \sigma_{\mathrm{id}}^{\Delta}\right\}$. By construction $\sigma_{\text {id }}^{\Delta} \leftarrow \mathrm{NH} . \operatorname{Sign}\left(\mathrm{sk}_{\mathrm{id}}^{\mathrm{NH}}, \mathrm{vk}_{\mathrm{id}}^{\Delta} \mid \Delta\right)$, and thus $\mathrm{NH} . \operatorname{Ver}\left(\mathrm{pk}_{\mathrm{id}}^{\mathrm{NH}}, \mathrm{vk}_{\mathrm{id}}^{\Delta} \mid \Delta, \sigma_{\text {id }}^{\Delta}\right)$ outputs 1 for the correctness of the NH.Sig. Similarly, being $\mathcal{P}$ the identity program $\mathcal{I}_{\ell}$, it holds that $\operatorname{Ver}^{\prime}\left(\mathcal{P},\left(\mathrm{pp}_{\mathrm{id}}, \mathrm{vk}_{\mathrm{id}}^{\Delta}\right), \mathrm{m}, \sigma^{\prime}\right)=1$ by the correctness of the MKHSig scheme.

\footnotetext{
${ }^{10}$ Here we assume that a $\rho$-bits string is sufficient, otherwise it can always be stretched using a PRG.
} 
Evaluation Correctness. Evaluation correctness follows directly from the correctness of the evaluation algorithm Eval' of the single-dataset MKHSig scheme, the correctness of NH.Sig and of the PRF.

Security. Intuitively, the security of the scheme follows from two main observations. First, no adversary is able to fake the keys of the single-dataset multi-key homomorphic signature scheme, due to the security of the standard signature scheme and the property of pseudo-random functions. Secondly, no adversary can tamper with the results of Eval for a specific dataset as this would correspond to breaking the security of the single-dataset multi-key homomorphic signature scheme.

Theorem 18. If $F$ is a secure pseudo-random function, NH.Sig is an unforgeable

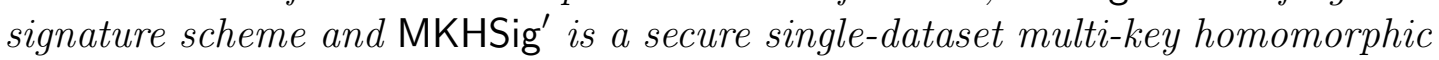
signature scheme, then the MKHSig scheme for multiple datasets described in Section 4.5 .3 is secure against adversaries that make static corruptions of keys and produce forgeries as in Definition 18 .

In order to prove Theorem 18 we define a sequence of hybrid games that we will use to bound the probability of winning in the original security game described in Section 4.4. Let Game $\mathbf{0}$ be the security game between the adversary $\mathcal{A}$ and the challenger $\mathcal{C}$ described in Section 4.4. Let us define two more hybrid games:

Game 1 This is the same as Game 0 except that every PRF instance $F_{\mathrm{K}_{\text {id }}}(\cdot)$ is replaced with a truly random function $\mathcal{R}_{\text {id }}:\{0,1\}^{*} \rightarrow\{0,1\}^{\rho}$. Basically, for every dataset identifier $\Delta$ used in the game, Game 1 generates keys $\left(\mathrm{sk}_{\mathrm{id}}^{\Delta}, \mathrm{ek}_{\mathrm{id}}^{\Delta}, \mathrm{vk}_{\mathrm{id}}^{\Delta}\right.$ ) using KeyGen ${ }_{2}$ using truly random coins as output by $\mathcal{R}_{\text {id }}(\Delta)$.

Game 2 This is the same as Game 1 except that the challenger rejects any adversary's forgery $\left(\mathcal{P}^{*}, \Delta^{*}, \mathrm{~m}^{*}, \sigma^{*}\right)$ where $\Delta^{*}$ had not been queried during the authentication query phase (i.e. $L_{\Delta^{*}}$ was not initialised). Note that in Game 2 the adversary can no longer make Type 1 forgeries.

Let $\mathrm{G}_{i}(\mathcal{A})$ denote the event that Game $i$, executed with adversary $\mathcal{A}$, outputs 1. The proof of Theorem 18 is obtained by showing that any PPT adversary has negligible probability of distinguishing between Game 0 and Game 1 (Lemma 22), and between Game 1 and Game 2 (Lemma 23). Finally, we show that $\mathcal{A}$ has negligible probability of winning in the final game (Lemma 24).

Lemma 22. For every PPT $\mathcal{A}$ there is a PPT distinguisher $\mathcal{D}$ such that $\mid \operatorname{Pr}\left[\mathrm{G}_{0}(\mathcal{A})\right]-$ $\operatorname{Pr}\left[\mathrm{G}_{1}(\mathcal{A})\right] \mid \leq Q_{\text {id }} \cdot \mathbf{A d v}_{F, \mathcal{D}}^{P R F}(\lambda)$, where $Q_{\text {id }}$ is the number of distinct identities queried by $\mathcal{A}$ during authentication queries.

The proof of Lemma 22 is rather simple and proceeds as follows. Given that the only difference between Game 0 and Game 1 is in the random coins used 
in algorithm $\mathrm{KeyGen}_{2}$, the probability of $\mathcal{A}$ winning in Game 1 is the same as in Game 0, a part from an additive factor that comes from distinguishing the PRF instance $F_{\mathrm{K}_{\text {id }}}(\cdot)$ from a truly random function $\mathcal{R}_{\text {id }}(\cdot)$. Since we assume $\mathcal{A}$ performs at most $Q_{\text {id }}$ distinct PRF instances during the game, a fairly standard hybrid argument assures that the difference between the two games is bound by a $Q_{\text {id }} \cdot \operatorname{Adv}_{F, \mathcal{D}}^{P R F}(\lambda)$ factor.

Lemma 23. For every PPT $\mathcal{A}$ there is a PPT forger $\mathbb{F}$ such that $\mid \operatorname{Pr}\left[\mathrm{G}_{1}(\mathcal{A})\right]-$ $\operatorname{Pr}\left[\mathrm{G}_{2}(\mathcal{A})\right] \mid \leq Q_{\mathrm{id}} \cdot \mathbf{A d v}_{\mathrm{NH} . \mathrm{Sig}, \mathbb{F}}^{\mathrm{UF}-\mathrm{CMA}}(\lambda)$, where $\mathbf{A d v}_{\mathrm{NH} . \mathrm{Sig}, \mathbb{F}}^{\mathrm{UF}}(\lambda)$ is the advantage of $\mathbb{F}$ breaking the existential unforgeability of the standard (non-homomorphic) signature scheme and $Q_{\mathrm{id}}$ is the number of distinct identities queried by $\mathcal{A}$.

For the proof of Lemma 23, we notice that the outputs of Game 1 and Game 2 coincide on every forgery $\left(\mathcal{P}^{*}, \Delta^{*}, \mathrm{~m}^{*}, \sigma^{*}\right)$ except when $\mathcal{A}$ produces a candidate Type 1 forgery, i.e., when $\Delta^{*}$ has never been queried during the game. Let Bad denote the event in which the adversary outputs a valid Type 1 forgery, then $\left|\operatorname{Pr}\left[G_{1}(\mathcal{A})\right]-\operatorname{Pr}\left[G_{2}(\mathcal{A})\right]\right|=\operatorname{Pr}\left[G_{1}(\mathcal{A}) \wedge \operatorname{Bad}\right]$. In what follows we bound this probability by showing that for every adversary $\mathcal{A}$ that wins in Game 1 by returning a Type 1 forgery there is a forger $\mathbb{F}$ that produces an existential forgery against the scheme NH.Sig.

Observe that the forgery $\left(\mathcal{P}^{*}, \Delta^{*}, \mathrm{~m}^{*}, \sigma^{*}\right)$ passes verification Ver of the multidataset MKHSig and that $\mathcal{P}^{*}$ contains at least one identity. Fix any identity id $\in \mathcal{P}^{*}$ and let it be the $k$-th identity that $\mathcal{A}$ queried in the game. The crucial observation is that since $\Delta^{*}$ has never been queried, the forgery contains a signature $\sigma_{\mathrm{i} \overline{\mathrm{d}}}^{\Delta^{*}}$ which was not generated by the challenger and yet verifies correctly under the public key $\mathrm{pk}_{\mathrm{id}}^{\mathrm{NH}}$. Therefore, for any such adversary $\mathcal{A}$, we can build a PPT $\mathbb{F}$ which wins in the unforgeability game of NH.Sig with non-negligible probability. The forger $\mathbb{F}$ works as follows. First, $\mathbb{F}$ samples a random index $k^{*} \stackrel{\$}{\leftarrow}\left[Q_{\text {id }}\right]$ which represents its guess for the index of the identity id on which $\mathcal{A}$ will make the forgery (i.e., $k^{*}$ is a guess for the above $k$ ). Second, $\mathbb{F}$ simulates Game 1 to $\mathcal{A}$ by generating the keys of the NH.Sig scheme for all identities except the $k^{*}$-th one, for which $\mathbb{F}$ invokes its own oracles for the simulation. The forger $\mathbb{F}$ also generates all the keys of the multi-key homomorphic scheme necessary in the simulation.

Then, let $\left(\mathcal{P}^{*}, \Delta^{*}, \mathrm{~m}^{*}, \sigma^{*}\right)$ be the Type 1 forgery returned by $\mathcal{A}$, where $\sigma^{*}=$ $\left(\mathrm{I}^{*}, \sigma^{\prime}, \operatorname{par}_{\Delta^{*}}\right)$ and $\operatorname{par}_{\Delta^{*}}=\left\{\left(\mathrm{ek}_{\mathrm{id}}^{\Delta^{*}}, \mathrm{vk}_{\mathrm{id}}^{\Delta^{*}}, \sigma_{\mathrm{id}}^{\Delta^{*}}\right)\right\}_{\mathrm{id} \in \mathrm{I}^{*}}$. If the $k^{*}$-th identity id is in $\mathcal{P}^{*}$ then $\sigma_{\overline{\mathrm{id}}}^{\Delta^{*}}$ is such that $\mathrm{NH} . \operatorname{Ver}\left(\mathrm{pk}_{\mathrm{id}}^{\mathrm{NH}},\left(\mathrm{vk}_{\overline{\mathrm{id}}}^{\Delta} \mid \Delta\right), \sigma_{\overline{\mathrm{id}}}^{\Delta^{*}}\right)=1$, i.e., the signature $\sigma_{\overline{\mathrm{id}}}^{\Delta^{*}}$ verifies correctly on the message $\left(\mathrm{vk}_{\mathrm{i} \overline{\mathrm{d}}}^{\Delta^{*}} \mid \Delta^{*}\right)$. Since no message $\left(\cdot \mid \Delta^{*}\right)$ was queried by $\mathbb{F}$ to its oracle, the pair $\left(\left(\mathrm{vk}_{\mathrm{i \textrm {d }}}^{\Delta} \mid \Delta\right), \sigma_{\overline{\mathrm{id}}}^{\Delta^{*}}\right)$ is an existential forgery for NH.Sig. In case the $k^{*}$-th identity id is not in $\mathcal{P}^{*}$, the forger $\mathbb{F}$ aborts the simulation.

In conclusion if $\operatorname{Pr}\left[\mathrm{G}_{1}(\mathcal{A}) \wedge \mathrm{Bad}\right]>\epsilon$, then $\mathbb{F}$ has advantage $>\epsilon / Q_{\text {id }}$ in breaking the security of NH.Sig, where the factor $1 / Q_{\text {id }}$ is the probability that $\mathbb{F}$ guesses correctly the index $k^{*}$, i.e., $\operatorname{Pr}\left[k=k^{*}\right]$. Since by assumption NH.Sig is an unforgeable 
signature scheme, we derive that $\left|\operatorname{Pr}\left[\mathrm{G}_{1}(\mathcal{A})\right]-\operatorname{Pr}\left[\mathrm{G}_{2}(\mathcal{A})\right]\right|=\operatorname{Pr}\left[\mathrm{G}_{1}(\mathcal{A}) \wedge \operatorname{Bad}\right] \leq$ $Q_{\text {id }} \cdot \operatorname{Adv}_{\text {NH.Sig, } \mathbb{F}}^{\mathrm{UF}-\mathrm{CMA}}(\lambda)$, which concludes the proof.

Lemma 24. For every PPT adversary $\mathcal{A}$ there is a PPT algorithm $\mathcal{B}$ such that $\operatorname{Pr}\left[\mathrm{G}_{2}(\mathcal{A})\right] \leq Q_{\Delta} \cdot \mathbf{A d v}_{\mathrm{MKHSig}^{\prime}, \mathcal{B}}^{\mathrm{HomUF}^{-C M A}}(\lambda)$, where $Q_{\Delta}$ is the number of distinct datasets queried by $\mathcal{A}$.

The intuition of the proof of Lemma 24 is the following: since Game 2 rules out all Type 1 forgeries, the only way in which $\mathcal{A}$ can win Game 2 is to produce a forgery on a single, previously queried dataset. In this case we will reduce the security of Game 2 to the standard security game of the single-dataset multi-key homomorphic signature.

We have already noticed that $\mathrm{G}_{2}(\mathcal{A})$ happens if and only if $\mathcal{A}$ produces a Type 2 or Type 3 forgery against the multi-dataset multi-client homomorphic signature scheme, where the pseudo random function $F$ is replaced by a random function $\mathcal{R}$. The latter change assures that for every new queried dataset the fresh generated keys for the MKHSig' scheme (obtained from KeyGen ${ }_{2}$ ) have the same distribution as the keys generated in the standard security game of MKHSig'.

In what follows we show that every Type 2 or Type 3 forgery produced by $\mathcal{A}$ in Game 2 can be used by an adversary $\mathcal{B}$ to win the security game for the single dataset scheme MKHSig' with a forgery of the same type for one of the $Q_{\Delta}$ instances of the single-dataset $\mathrm{MKHSig}$ ' scheme that were generated during Game 2. Fix a dataset $\bar{\Delta}^{*}$ and let it be the $k$-th dataset queried by $\mathcal{A}$ in Game 2. The adversary $\mathcal{B}$ proceeds as follows. It samples a random index $k^{*} \leftarrow\left[Q_{\Delta}\right]$ (which represents its guess for $k$ ), and then simulates Game 2 to $\mathcal{A}$ by choosing the keys of the MKHSig' on its own for all datasets $k \neq k^{*}$, whereas for the $k^{*}$-th dataset $\mathcal{B}$ does the simulation forwarding the queries to its challenger.

Let $\left(\mathcal{P}^{*}, \Delta^{*}, \mathrm{~m}^{*}, \sigma^{*}\right)$ be a valid Type 2 (resp. Type 3 ) forgery returned by $\mathcal{A}$ where $\sigma^{*}=\left(\mathrm{I}^{*}, \sigma^{\prime}, \operatorname{par}_{\Delta^{*}}\right)$. If $\Delta^{*}$ is not the $k^{*}$-th queried dataset, $\mathcal{B}$ aborts. Otherwise, $\Delta^{*}$ is the $k^{*}$-th queried dataset, i.e. $\Delta^{*}=\bar{\Delta}^{*}$, and the second entry of $\sigma^{*}=\left(\mathbf{I}^{*}, \sigma^{\prime}, \operatorname{par}_{\bar{\Delta}^{*}}\right)$ satisfies $\operatorname{Ver}^{\prime}\left(\mathcal{P}^{*}, \bar{\Delta}^{*}, \mathrm{~m}^{*}, \sigma^{\prime}\right)=1$. The last equations implies that $\left(\mathcal{P}^{*}, \bar{\Delta}^{*}, \mathrm{~m}^{*}, \sigma^{\prime}\right)$ is a valid Type 2 (resp. Type 3 ) forgery against the MKHSig' scheme.

To conclude, we notice that if $\operatorname{Pr}\left[\mathrm{G}_{2}(\mathcal{A})\right]>\epsilon$, then $\mathcal{B}$ has at least $\epsilon / Q_{\Delta}$ advantage in breaking the security of $\mathrm{MKHSig}^{\prime}$, where the factor $1 / Q_{\Delta}=\operatorname{Pr}\left[k=k^{*}\right]$ is the probability of $\mathcal{B}$ guessing correctly the index $k^{*}$ of the database $\Delta^{*}$ used in by $\mathcal{A}$ in the forgery.

Combining the results of the three lemmas above, we obtain the following bound:

$$
\operatorname{Pr}[\mathrm{G}(\mathcal{A})] \leq Q_{\mathrm{id}} \cdot \mathbf{A d v}_{F, \mathcal{D}}^{P R F}(\lambda)+Q_{\text {id }} \cdot \mathbf{A d v}_{\mathrm{NH} . \operatorname{Sig}, \mathbb{F}}^{\mathrm{UF}-\mathrm{CMA}}(\lambda)+Q_{\Delta} \cdot \mathbf{A d v}_{\mathrm{MKHSig}^{\prime}, \mathcal{B}}^{\operatorname{HomUF-CMA}}(\lambda)
$$

which proves the statement of Theorem 18 , since all the addends are negligible by assumption. 


\subsubsection{Generic Transformations from Weak to Adaptive Security}

In this section we present two generic transformations that turn weakly secure multi-key HA schemes into adaptive secure ones.

Our first transformation holds in the standard model and works for schemes in which the size of the tag space $\mathcal{T}$ is at most polynomial in the security parameter. The second transformation avoids the limitation on the size of the tag space (i.e., it works for schemes that can handle tags that are binary strings of virtually arbitrary length), but holds in the random oracle model. Both transformations rely on the notion of homomorphic trapdoor functions (HTDFs) introduced in [77.

In what follows, we first recall the notion of HTDFs and subsequently describe our transformations. Moreover it is straightforward to see that both transformations extend to the case of multi-key homomorphic signatures.

Homomorphic Trapdoor Functions [77]. The concept of homomorphic trapdoor functions was recently introduced by Gorbunov et al. in [77]. Intuitively, an homomorphic trapdoor function $f$ is defined by a public key pk which allows to compute $v=f_{\mathrm{pk}, x}(u)$ on input $u$ and index $x$. The homomorphic property enables anyone with knowledge of triples $\left\{u_{i}, x_{i}, v_{i}=f_{\mathrm{pk}, x_{i}}\left(u_{i}\right)\right\}_{i \in[t]}$ and a $t$-input function $g$ to generate values $u^{*}, v^{*}$ such that $v^{*}=f_{\mathrm{pk}, g\left(x_{1}, \ldots, x_{t}\right)}\left(u^{*}\right)$. More formally:

Definition 22 (Homomorphic Trapdoor Functions [77]). An homomorphic trapdoor function (HTDF) is a tuple of five PPT algorithms HTDF $=$ (HTDF.KeyGen, $f$, Inv, HTDF.Eval ${ }^{\text {in }}$, HTDF.Eval ${ }^{\text {out }}$ ) which are defined as follows:

HTDF.KeyGen $\left(1^{\lambda}\right)$. The key generation algorithm takes as input a security parameter $\lambda$ and outputs a key-pair (pk,sk). The public key implicitly defines the index space $\mathcal{X}$, the input space $\mathcal{U}$, the output space $\mathcal{V}$ and some efficiently samplable input distribution $\mathcal{D}_{\mathcal{U}}$ over $\mathcal{U}$. Testing membership in the sets $\mathcal{U}, \mathcal{V}, \mathcal{X}$ and sampling uniformly at random in $\mathcal{V}$ are required to be efficiently doable.

$f_{\mathrm{pk}, x}(u)$. This is a deterministic function, indexed by $\mathrm{pk}$ and $x \in \mathcal{X}$. It takes as input an element $u \in \mathcal{U}$ and outputs an element $v \in \mathcal{V}$. Note that the function $f$ is not required to be injective.

$\operatorname{Inv}_{\mathbf{s k}, x}(v)$. This is a probabilistic algorithm which is indexed by sk and $x \in \mathcal{X}$. It takes as input an element $v \in \mathcal{V}$ and outputs an element $u \in \mathcal{U}$.

HTDF.Eval ${ }_{\mathrm{pk}}^{i n}\left(g,\left(x_{1}, u_{1}\right), \ldots,\left(x_{t}, u_{t}\right)\right)$. The input evaluation algorithm takes as input a function $g: \mathcal{X}^{t} \rightarrow \mathcal{X}$ along with a collection of pairs $\left\{\left(x_{i}, u_{i}\right)\right\}_{i=1}^{t} \in$ $\mathcal{X} \times \mathcal{U}$ and outputs a value $u^{*} \in \mathcal{U}$. 
HTDF.Eval $\mathrm{pk}_{\mathrm{pk}}^{\text {ut }}\left(g, v_{1}, \ldots, v_{t}\right)$. The output evaluation algorithm takes as input a function $g: \mathcal{X}^{t} \rightarrow \mathcal{X}$ and elements $\left\{v_{i}\right\}_{i=1}^{t} \in \mathcal{V}$, and outputs a value $v^{*} \in \mathcal{V}$.

Additionally, an HTDF is required to satisfy the properties of correctness, distributional equivalence of inversion and security, as described below.

Correctness of Evaluation Procedure. Let (pk, sk) $\leftarrow$ HTDF.KeyGen $\left(1^{\lambda}\right)$, $x_{1}, \ldots, x_{t} \in \mathcal{X}, g: \mathcal{X}^{t} \rightarrow \mathcal{X}$ and $y=g\left(x_{1}, \ldots, x_{t}\right)$. For all $i \in[t]$, let $u_{i} \in \mathcal{U}$ and $v_{i} \in \mathcal{V}$ such that $v_{i}=f_{\mathrm{pk}, x_{i}}\left(u_{i}\right)$. If $u^{*}:=\operatorname{HTDF}$.Eval $\left.\right|_{\mathrm{pk}} ^{i n}\left(g,\left(x_{1}, u_{1}\right), \ldots,\left(x_{t}, u_{t}\right)\right)$ and $v^{*}:=\operatorname{HTDF}$. Eval $\left._{\mathrm{pk}}^{\text {out }}\left(g, v_{1}, \ldots, v_{t}\right)\right)$, then it must be $u^{*} \in \mathcal{U}$ and $f_{\mathrm{pk}, y}\left(u^{*}\right)=v^{*}$.

Distributional Equivalence of Inversion. Let (pk, sk $\leftarrow$ HTDF.KeyGen $\left(1^{\lambda}\right)$, $x \in \mathcal{X}, u \stackrel{\$}{\leftarrow} \mathcal{D}_{\mathcal{U}}, v=f_{\mathrm{pk}, x}(u), v^{\prime} \stackrel{\$}{\leftarrow} \mathcal{V}$ and $u^{\prime} \leftarrow \operatorname{lnv}_{\mathrm{sk}, x}\left(v^{\prime}\right)$. Then, we require that

$$
(\mathrm{pk}, \mathrm{sk}, x, u, v) \stackrel{\text { stat }}{\approx}\left(\mathrm{pk}, \mathrm{sk}, x, u^{\prime}, v^{\prime}\right)
$$

HTDF Security. For security, HTDFs are required to satisfy a property similar to claw-freeness. Intuitively, it should be difficult for any adversary to come up with $u, u^{\prime} \in \mathcal{U}$ and $x, x^{\prime}$ such that $x \neq x^{\prime}$ and $f_{\mathrm{pk}, x}(u)=f_{\mathrm{pk}, x^{\prime}}\left(u^{\prime}\right)$. More formally, for any PPT adversary $\mathcal{A}$ it holds

$$
\operatorname{Pr}\left[\begin{array}{c|c}
f_{\mathrm{pk}, x}(u)=f_{\mathrm{pk}, x^{\prime}}\left(u^{\prime}\right) & (\mathrm{pk}, \mathrm{sk}) \leftarrow \operatorname{HTDF} . K e y G e n\left(1^{\lambda}\right) \\
u, u^{\prime} \in \mathcal{U}, \quad x, x^{\prime} \in \mathcal{X}, x \neq x^{\prime} & \left(u, u^{\prime}, x, x^{\prime}\right) \leftarrow \mathcal{A}\left(1^{\lambda}, \mathrm{pk}\right)
\end{array}\right] \leq \operatorname{negl}(\lambda) .
$$

Our Transformation for Polynomially-Large Tag Spaces. Before showing the transformation in details, we provide a general intuition of the approach. First, we assume to start with a weakly-secure multi-key HA scheme MKHAut ${ }^{\prime}$ and an homomorphic trapdoor function HTDF. The idea of the transformation is that HTDFs are essentially homomorphic chameleon hash functions. Hence, one can follow the standard hash-and-sign approach based on chameleon hashes, which is known to provide a weak-to-adaptive security transformation for digital signatures. To authenticate a message $\mathrm{m}$ with the new adaptive secure scheme MKHAut one chooses $u \stackrel{\$}{\leftarrow} \mathcal{D}_{\mathcal{U}}$ from the input distribution $\mathcal{D}_{\mathcal{U}}$ of $\mathrm{F}$, computes $v \leftarrow f_{\mathrm{pk}, \mathrm{m}}(u)$ and finally authenticates $v$ using MKHAut' thus obtaining an authenticator $\bar{\sigma}$. The final authenticator is $\sigma=(v, u, \bar{\sigma})$. To homomorphically evaluate a function $g: \mathcal{M}^{t} \rightarrow \mathcal{M}$ over such authenticators one first computes $u^{*} \leftarrow \operatorname{HTDF}_{\text {Eval }}^{\text {in }}\left(g,\left(\mathrm{~m}_{1}, u_{1}\right), \ldots,\left(\mathrm{m}_{t}, u_{t}\right)\right)$ and $v^{*} \leftarrow \operatorname{HTDF}_{\mathrm{pval}}^{\mathrm{out}}\left(g, v_{1}, \ldots, v_{t}\right)$. Next, the idea is to use MKHAut ${ }^{\prime}$ to vouch for the correctness of $v^{*}$ as the output of a function $g^{\prime}(\cdots)=\mathrm{HTDF}_{\mathrm{Eval}}^{\mathrm{out}}(g, \cdots)$. Namely, one executes the homomorphic evaluation algorithm of MKHAut ${ }^{\prime}$ over $\left\{\bar{\sigma}_{i}\right\}_{i \in t}$ with function $g^{\prime}$. In terms of security, the intuition is that in order to break the security of the new scheme one has to either find a claw in the HTDF, or break the security of MKHAut' to cheat on a false $v^{\prime} \neq v^{*}$. It is also worth observing that, due to the property of 
HTDFs, MKHAut $^{\prime}$ is essentially used to authenticate data $-v-$ that is random and independent of the real message $\mathrm{m}$.

We describe our transformation more formally below.

Our first transformation. Let $\mathrm{F}=$ (HTDF.KeyGen, $f$, Inv, HTDF.Eval ${ }^{\text {in }}$, HTDF. Eval $^{\text {out }}$ ) be an HTDF with index space $\mathcal{X}$, input space $\mathcal{U}$ (with distribution $\mathcal{D}_{\mathcal{U}}$ ), and output space $\mathcal{V}$. Let MKHAut ${ }^{\prime}=\left(\right.$ Setup $^{\prime}$, KeyGen $^{\prime}$, Auth $^{\prime}$, Eval $^{\prime}$, Ver $\left.^{\prime}\right)$ be a (weakly-secure) multi-key homomorphic authentication scheme. Without loss of generality, we will assume the message space of MKHAut ${ }^{\prime}$ to be $\mathcal{V}$. We also assume that the tag space $\mathcal{T}$ of MKHAut' is of polynomial size, i.e., $\mathrm{T}:=|\mathcal{T}|=\operatorname{poly}(\lambda)$. Using these tools we construct an adaptive secure homomorphic authenticator MKHAut $=$ (Setup, KeyGen, Auth, Eval, Ver) with message space $\mathcal{M}=\mathcal{X}$. The scheme works as follows:

$\operatorname{Setup}\left(1^{\lambda}\right)$. Generate $\mathrm{pp}^{\prime} \leftarrow \operatorname{Setup}^{\prime}\left(1^{\lambda}\right)$ and $\left(\mathrm{pk}_{h}, \mathrm{sk}_{h}\right) \leftarrow$ HTDF.KeyGen $\left(1^{\lambda}\right)$, and output $\mathrm{pp}=\left(\mathrm{pp}^{\prime}, \mathrm{pk}_{h}\right)$.

$\operatorname{KeyGen}(\mathrm{pp})$. Generate $\left(a k^{\prime}, \mathrm{ek}^{\prime}, \mathrm{vk}^{\prime}\right) \leftarrow \operatorname{KeyGen}\left(\mathrm{pp}^{\prime}\right)$, and output $a \mathrm{k}=\mathrm{ak} \mathbf{k}^{\prime}$, ek $=$ $\mathrm{ek}^{\prime}$, and $\mathrm{vk}=\mathrm{vk}^{\prime}$.

Auth(ak, $\Delta, \ell, \mathrm{m})$. Sample $u \stackrel{\$}{\leftarrow} \mathcal{D}_{\mathcal{U}}$, compute $v=f_{\mathrm{pk}_{h}, \mathrm{~m}}(u)$, and finally compute $\bar{\sigma} \leftarrow \operatorname{Auth}^{\prime}\left(\mathrm{ak}^{\prime}, \Delta, \ell, v\right)$ and output $\sigma:=(v, u, \mathrm{~m}, \bar{\sigma})$.

$\operatorname{Eval}\left(g,\left\{\sigma_{i}, \mathrm{EKS}_{i}\right\}_{i \in[t]}\right)$. Given the function $g: \mathcal{M}^{t} \rightarrow \mathcal{M}$, we define a corresponding function $g^{\prime}: \mathcal{V}^{t} \rightarrow \mathcal{V}$ as $g^{\prime}\left(v_{1}, \ldots, v_{t}\right)=\operatorname{HTDF}$.Eval ${ }_{\mathrm{pk}_{h}}^{\text {out }}\left(g, v_{1}, \ldots, v_{t}\right)$. For all $i \in[t]$, parse every authenticator $\sigma_{i}=\left(v_{i}, u_{i}, \mathrm{~m}_{i}, \bar{\sigma}_{i}\right)$. Then, the evaluation algorithm computes $\mathrm{m}=g\left(\mathrm{~m}_{1}, \ldots, \mathrm{m}_{t}\right), v \leftarrow \operatorname{HTDF}_{\text {Eval }} \mathrm{pk}_{h}^{\text {out }}\left(g, v_{1}\right.$, $\left.\ldots, v_{t}\right), u \leftarrow$ HTDF.Eval ${ }_{\mathrm{pk}_{h}}^{i n}\left(g,\left(\mathrm{~m}_{1}, u_{1}\right), \ldots,\left(\mathrm{m}_{t}, u_{t}\right)\right)$ and $\bar{\sigma} \leftarrow \operatorname{Eval}^{\prime}\left(g^{\prime},\left\{\bar{\sigma}_{i}\right.\right.$, $\left.\left.\left.\mathrm{EKS}_{i}\right)\right\}_{i=1}^{t}\right)$. Finally it outputs $\sigma=(v, u, \mathrm{~m}, \bar{\sigma})$.

$\operatorname{Ver}\left(\mathcal{P}:=\left(g, \ell_{1}, \ldots, \ell_{t}\right), \Delta,\left\{\mathrm{vk}_{\mathrm{id}}\right\}_{\mathrm{id} \in \mathcal{P}^{\prime}}, \mathrm{m}, \sigma\right)$. Parse $\sigma:=(v, u, \mathrm{~m}, \bar{\sigma})$, and output 1 if both $f_{\mathrm{pk}_{h}, \mathrm{~m}}(u)=v$ and $\operatorname{Ver}^{\prime}\left(\mathcal{P}^{\prime}:=\left(g^{\prime}, \ell_{1}, \ldots, \ell_{t}\right), \Delta,\left\{\mathrm{vk}_{\mathrm{id}}\right\}_{\mathrm{id} \in \mathcal{P}}, v, \bar{\sigma}\right)=1$ hold. Otherwise output 0.

It is easy to see that the correctness of MKHAut follows from the correctness of both MKHAut' and the HTDFF.

Theorem 19. If $\mathrm{F}$ is a secure HTDF, MKHAut' is a weakly secure multi-key homomorphic authenticator whose tag space $\mathcal{T}$ is such that $|\mathcal{T}|=\operatorname{poly}(\lambda)$, then the scheme MKHAut defined above is an adaptively secure multi-key homomorphic authenticator.

The proof proceeds by contradiction. Namely, assuming the existence of an adversary $\mathcal{A}$ which breaks the adaptive security of MKHAut we show that one can break either the security of the HTDF or the weak security of MKHAut'. To see this, let $\mathcal{A}$ be an adversary that wins in the adaptive security game described 
in Definition 17, and let $\left(\mathcal{P}^{*}:=\left(g^{*}, \ell_{1}^{*}, \ldots, \ell_{t}^{*}\right), \Delta^{*}, \mathrm{~m}^{*}, \sigma^{*}:=\left(v^{*}, u^{*}, \mathrm{~m}^{*}, \bar{\sigma}^{*}\right)\right)$ be its forgery. We observe that $\sigma^{*}$ can be of either one of the following types:

Type 1. As per Definition 17, the list $L_{\Delta^{*}}$ has never been initialized during the game.

Type 2. $\forall i \in[t]$ we have $\left(\ell_{i}^{*}, \mathrm{~m}_{i}\right) \in L_{\Delta^{*}}$, but $\mathrm{m}^{*} \neq g^{*}\left(\mathrm{~m}_{1}, \ldots, \mathrm{m}_{t}\right)$. If we let $\left\{\sigma_{i} \leftarrow \operatorname{Auth}\left(\mathrm{sk}, \Delta^{*}, \ell_{i}^{*}, \mathrm{~m}_{i}\right)\right\}_{i \in[t]}$ be the authenticators given to $\mathcal{A}$ during the game, and let $\hat{\sigma}:=(\hat{v}, \hat{u}, \hat{\mathrm{m}}, \hat{\bar{\sigma}}) \leftarrow \operatorname{Eval}\left(g^{*},\left\{\sigma_{i}, \mathrm{EKS}_{i}\right\}_{i \in[t]}\right), \mathrm{m}:=$ $g^{*}\left(\mathrm{~m}_{1}, \ldots, \mathrm{m}_{t}\right)$. There are two sub-cases which can occur: either $(\mathrm{a}) v^{*} \neq \hat{v}$, or (b) $v^{*}=\hat{v}$.

Type 3. As per Definition 17 , there exists a label $\ell^{*}$ such that $\left(\ell^{*}, \cdot\right) \notin L_{\Delta^{*}}$.

We show that for every $\mathcal{A}$ producing forgeries of Type 1, 2.(a) or 3 there exists an adversary that breaks the weak security of MKHAut', whereas for every $\mathcal{A}$ producing a forgery of type 2.(b) it is possible to break the security of $\mathrm{F}$. More precisely, we provide a detailed reduction $\mathcal{B}$ which exploits any Type 1 or Type 2.(a) forgery made by $\mathcal{A}$ to break the security of the MKHAut' scheme. We then notice that a similar reduction $\mathcal{B}^{\prime}$ holds for Type 3 forgeries. Theorem 19 is proven by combining the results of Lemmas 25, 26 and 27 given below.

In the following, we set $Q=\operatorname{poly}(\lambda)$ to be the total number of queries made by $\mathcal{A}$, similarly $Q_{\text {id }}, Q_{\mathcal{T}}, Q_{\Delta}$ will be respectively the number of distinct identities, tags and datasets queried by $\mathcal{A}$ during the authentication-query phase (note that $\left.Q_{\text {id }}, Q_{\mathcal{T}}, Q_{\Delta} \leq Q\right)$.

Lemma 25. If $\mathcal{A}$ is an adversary that has non-negligible advantage $\epsilon$ against the security of MKHAut by producing a Type 1 (respectively Type 2.(a)) forgery, then it is possible to build an adversary $\mathcal{B}$ that has advantage $\epsilon$ against the weak security of MKHAut' by producing a Type 1 (respectively Type 2) forgery.

Proof. We want here to build a reduction $\mathcal{B}$, which uses a Type 1 or 2.(a) forgery by $\mathcal{A}$ to break the security of MKHAut'. Let $\mathcal{T}:=\left\{\tau_{1}, \ldots, \tau_{\mathrm{T}}\right\}$ where $\mathrm{T}=\operatorname{poly}(\lambda)$.

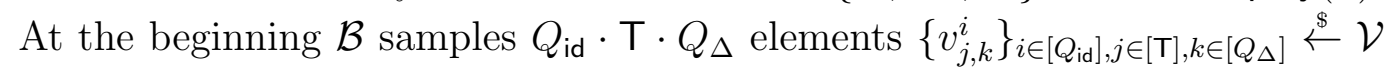
and chooses a pair of keys $\left(\mathrm{pk}_{h}, \mathrm{sk}_{h}\right)$ for F. Following the weak-security definition, $\mathcal{B}$ sends $\left\{\left(v_{j, k}^{i}, \tau_{j}\right)\right\}_{(i, j, k) \in\left[Q_{\text {id }}\right] \times[\mathrm{T}] \times\left[Q_{\Delta}\right]}$ to its challenger $\mathcal{C}$ and gets back the public parameters $\mathrm{pp}^{\prime}$ of MKHAut'. Next, $\mathcal{B}$ sets $\mathrm{pp}=\left(\mathrm{pp}^{\prime}, \mathrm{pk}_{h}\right)$, and gives $\mathrm{pp}$ to $\mathcal{A}$. Afterwards, $\mathcal{A}$ starts making authentication queries $(\Delta, \ell:=($ id,$\tau), \mathrm{m})$. For all such queries, let $\Delta_{1}, \ldots, \Delta_{Q_{\Delta}}$ be all the distinct dataset names queried, in order, by $\mathcal{A}$. Similarly, let id $_{1}, \ldots$, id $_{Q_{\text {id }}}$ be the distinct identities queried, in order, by $\mathcal{A}$ in the whole game.

To every authentication query $\left(\Delta, \ell:=(\mathrm{id}, \tau)\right.$, m) such that $\Delta=\Delta_{k}$ (i.e., $\Delta$ is the $k$-th queried dataset), id $=\mathrm{id}_{i}$ (i.e., id is the $i$-th queried identity) and $\tau=$ $\tau_{j} \in \mathcal{T}, \mathcal{B}$ answers as follows. First it queries $\mathcal{C}$ with the pair (id, $\Delta$ ) and obtains the authenticators $\left\{\bar{\sigma}_{1, k}^{i}, \ldots, \bar{\sigma}_{\mathrm{T}, k}^{i}\right\}$ corresponding to the values $\left\{v_{1, k}^{i}, \ldots, v_{\mathrm{T}, k}^{i}\right\}$. 
If (id, $\Delta$ ) were already queried to $\mathcal{C}$, then $\mathcal{B}$ uses the values obtained earlier. Second, $\mathcal{B}$ uses the inversion property of HTDFs in order to compute $u_{j, k}^{i} \leftarrow$ $\operatorname{lnv}_{\mathbf{s k}_{h}, \mathrm{~m}}\left(v_{j, k}^{i}\right)$, and finally sends to $\mathcal{A}$ the authenticator $\sigma:=\left(v_{j, k}^{i}, u_{j, k}^{i}, \mathrm{~m}, \bar{\sigma}_{j, k}^{i}\right)$. By the distributional equivalence of inversion property of HTDFs this simulation is statistically close to the real game. For what regards verification queries, any of them from $\mathcal{A}$ is sent to the challenger and the answer is then sent back to $\mathcal{A}$ itself. When $\mathcal{A}$ produces a forgery $\left(\mathcal{P}^{*}:=\left(g^{*}, \ell_{1}^{*}, \ldots, \ell_{t}^{*}\right), \Delta^{*}, \mathrm{~m}^{*}, \sigma^{*}:=\left(v^{*}, u^{*}, \mathrm{~m}^{*}, \bar{\sigma}^{*}\right)\right)$ $\mathcal{B}$ proceeds as follows:

Type 1. If this is the case, it is easy to see that $\left(\mathcal{P}^{\prime}:=\left(\left(g^{*}\right)^{\prime}, \ell_{1}^{*}, \ldots, \ell_{t}^{*}\right), \Delta^{*}, v^{*}, \bar{\sigma}^{*}\right)$ is a Type 1 forgery for MKHAut'.

Type 2.(a). Since in this case it holds $v^{*} \neq \hat{v}$, the tuple $\left(\mathcal{P}^{\prime}:=\left(\left(g^{*}\right)^{\prime}, \ell_{1}^{*}, \ldots\right.\right.$, $\left.\left.\ell_{t}^{*}\right), \Delta^{*}, v^{*}, \bar{\sigma}^{*}\right)$ is a Type 2 forgery for MKHAut'.

Therefore, if $\mathcal{A}$ has non-negligible probability $\epsilon$ of coming up with a Type 1 or Type 2.(a) forgery, then $\mathcal{B}$ has probability $\epsilon$ of coming up with a Type 1 or Type 2.(a) forgery against MKHAut'.

Lemma 26. If $\mathcal{A}$ is an adversary that has non-negligible advantage $\epsilon$ against the security of MKHAut by producing a Type 3 forgery, then it is possible to build an adversary $\mathcal{B}^{\prime}$ that has non-negligible advantage $\epsilon /\left(Q_{\mathrm{id}} \cdot \mathrm{T} \cdot Q_{\Delta}\right)$ against the weak security of MKHAut' by producing a Type 3 forgery.

Proof. The reduction $\mathcal{B}^{\prime}$ works similarly to the one in Lemma 25. The game starts with $\mathcal{B}^{\prime}$ sampling $Q_{\text {id }} \cdot \mathbf{T} \cdot Q_{\Delta}$ elements $\left\{\left\{v_{j, k}^{i}\right\}_{i \in\left[Q_{\text {id }}\right], j \in[\mathrm{T}], k \in\left[Q_{\Delta}\right]}\right\} \stackrel{\$}{\leftarrow} \mathcal{V}$. Next $\mathcal{B}^{\prime}$ chooses a triple $(\bar{i}, \bar{j}, \bar{k}) \stackrel{\$}{\leftarrow}\left[Q_{\text {id }}\right] \times[\mathrm{T}] \times\left[Q_{\Delta}\right]$, and generates keys $\left(\mathrm{pk}_{h}, \mathrm{sk}_{h}\right)$ for the HTDF function F.

Next, $\mathcal{B}^{\prime}$ sends to its challenger $\mathcal{C}$ the values $\left\{\left(v_{j, k}^{i}, \tau_{j}\right)\right\}_{(i, j, k) \in\left\{\left[Q_{\text {id }}\right] \times[\mathbf{T}] \times\left[Q_{\Delta}\right]\right\} \backslash(\bar{i}, \bar{j}, \bar{k})}$. Basically, this is the same as in the proof of Lemma 25 except that $\mathcal{B}^{\prime}$ does not send any value related to the triple $(\bar{i}, \bar{j}, \bar{k})$. Intuitively, this can be interpreted as $\mathcal{B}^{\prime}$ betting on the dataset $\Delta_{\bar{k}}$ and the label $\ell=\left(\mathrm{id}_{\bar{i}}, \tau_{\bar{j}}\right)$ over which the adversary $\mathcal{A}$ will make the Type 3 forgery.

$\mathcal{B}^{\prime}$ answers the queries of $\mathcal{A}$ exactly as in Lemma 25 except if $\mathcal{A}$ makes a query $(\Delta, \ell:=(\mathrm{id}, \tau), \mathrm{m})$ such that $\Delta=\Delta_{\bar{k}}, \mathrm{id}=\mathrm{id}_{\bar{i}}$ and $\tau=\tau_{\bar{j}}$. If this case occurs $\mathcal{B}^{\prime}$ aborts.

Let $\left(\mathcal{P}^{*}:=\left(g^{*}, \ell_{1}^{*}, \ldots, \ell_{t}^{*}\right), \Delta^{*}, \mathrm{~m}^{*}, \sigma^{*}:=\left(v^{*}, u^{*}, \mathrm{~m}^{*}, \bar{\sigma}^{*}\right)\right)$ be $\mathcal{A}$ 's forgery, and following our assumption let it be of Type 3. Namely there exists a label $\ell^{*}=$ $\left(\mathrm{id}^{*}, \tau^{*}\right) \in\left\{\ell_{1}^{*}, \ldots, \ell_{t}^{*}\right\}$ such that $\left(\ell^{*}, \cdot\right) \notin L_{\Delta^{*}}$. If $\Delta^{*} \neq \Delta_{\bar{k}}$, or id $\neq \mathrm{id}_{\bar{i}}$ or $\tau^{*} \neq \tau_{\bar{j}}$ then $\mathcal{B}^{\prime}$ aborts. Otherwise, it returns $\left(\mathcal{P}^{\prime}:=\left(\left(g^{*}\right)^{\prime}, \ell_{1}^{*}, \ldots, \ell_{t}^{*}\right), \Delta^{*}, v^{*}, \bar{\sigma}^{*}\right)$ as a forgery for MKHAut'.

Let bad be the event that $\mathcal{B}^{\prime}$ aborts in the simulation. If bad does not happen $\mathcal{B}^{\prime}$ has the same advantage as $\mathcal{A}$ in producing a Type 3 forgery. 
Therefore, what is left to show is an estimation of $\operatorname{Pr}[\neg$ bad $]$. However, it is not hard to see that, before bad occurs, the random choice of $(\bar{i}, \bar{j}, \bar{k}) \stackrel{\$}{\leftarrow}\left[Q_{\text {id }}\right] \times[\mathrm{T}] \times$ $\left[Q_{\Delta}\right]$ is perfectly hidden to $\mathcal{A}$. Hence, we have that $\operatorname{Pr}[\neg$ bad $]=\frac{1}{Q_{\text {id }} \cdot \mathrm{T} \cdot Q_{\Delta}}$ and thus $\mathcal{B}^{\prime}$ has advantage $\frac{\epsilon}{Q_{\text {id }} \cdot \mathrm{T} \cdot Q_{\Delta}}$, that is non-negligible as $\left(Q_{\text {id }} \cdot \mathrm{T} \cdot Q_{\Delta}\right)=\operatorname{poly}(\lambda)$.

Lemma 27. If $\mathcal{A}$ is an adversary that has non-negligible advantage $\epsilon$ against the security of MKHAut by producing a Type 2.(b) forgery, then it is possible to build an adversary $\mathcal{B}^{\prime \prime}$ which breaks the security of the HTDF F with non-negligible probability $\epsilon$.

Proof. The algorithm $\mathcal{B}^{\prime \prime}$ takes as input a public key $\mathrm{pk}_{h}$ of the homomorphic trapdoor function $\mathrm{F}$, and then starts simulating the adaptive security game to $\mathcal{A}$ by sampling all the secrets on its own - i.e., $\mathcal{B}^{\prime \prime}$ runs Setup and KeyGen - and giving $\mathrm{pp}=\left(\mathrm{pp}^{\prime}, \mathrm{pk}_{h}\right)$ to $\mathcal{A}$. It is easy to see that since $\mathcal{B}^{\prime \prime}$ has all the secret keys, it can perfectly simulate all queries to $\mathcal{A}$.

Let $\left(\mathcal{P}^{*}:=\left(g^{*}, \ell_{1}^{*}, \ldots, \ell_{t}^{*}\right), \Delta^{*}, \mathrm{~m}^{*}, \sigma^{*}:=\left(v^{*}, u^{*}, \mathrm{~m}^{*}, \bar{\sigma}^{*}\right)\right)$ be $\mathcal{A}$ 's forgery that, by the assumption in this lemma, is of Type 2.(b). Namely, if we let $\left\{\sigma_{i} \leftarrow\right.$ Auth(sk, $\left.\left.\Delta^{*}, \ell_{i}^{*}, \mathrm{~m}_{i}\right)\right\}_{i \in[t]}$ be the authenticators given to $\mathcal{A}$ in the experiment for dataset $\Delta^{*}$ and labels $\ell_{1}^{*}, \ldots, \ell_{t}^{*}$, and parse each $\sigma_{i}:=\left(v_{i}, u_{i}, \mathrm{~m}_{i}, \bar{\sigma}_{i}\right)$, it is the case that $\mathrm{m}^{*} \neq \hat{\mathrm{m}}$ and $v^{*}=\hat{v}$, where $\hat{v}=\leftarrow \operatorname{HTDF}$.Eval ${ }_{\mathrm{pk}_{h}}^{\text {out }}\left(g, v_{1}, \ldots, v_{t}\right)$ and $\hat{\mathrm{m}}=$ $g\left(\mathrm{~m}_{1}, \ldots, \mathrm{m}_{t}\right)$. Let $\hat{u} \leftarrow$ HTDF. Eval ${ }_{\mathrm{pk}_{h}}^{i n}\left(g,\left(\mathrm{~m}_{1}, u_{1}\right), \ldots,\left(\mathrm{m}_{t}, u_{t}\right)\right)$ and note that by correctness of the HTDF $\mathrm{F}$ we have that $f_{\mathrm{pk}_{h}, \hat{\mathrm{m}}}(\hat{u})=\hat{v}$ where $\hat{\mathrm{m}}=g\left(\mathrm{~m}_{1}, \ldots, \mathrm{m}_{t}\right)$. Moreover, since the forgery verifies correctly we also have $f_{\mathrm{pk}_{h}, \mathrm{~m}^{*}}\left(u^{*}\right)=v^{*}$.

One can see that $\mathcal{B}^{\prime \prime}$ has values $\hat{u}, u^{*} \in \mathcal{U}, \mathrm{m}, \hat{\mathrm{m}} \in \mathcal{X}$ such that $\mathrm{m} \neq \hat{\mathrm{m}}$ and $f_{\mathrm{pk}_{h}, \mathrm{~m}^{*}}\left(u^{*}\right)=f_{\mathrm{pk}_{h}, \hat{\mathrm{m}}}(\hat{u})$, i.e., a tuple that breaks the security of the HTDF.

Therefore, if $\mathcal{A}$ has non-negligible advantage $\epsilon$ in producing a forgery of Type 2.(b), then $\mathcal{B}^{\prime \prime}$ has non-negligible probability $\epsilon$ of breaking the security of the HTDF.

Our Transformation for Arbitrary Tag Spaces. Here we present our second transformation for converting a multi-key homomorphic signature from weak to adaptive security, now without limitations on the size of the tag space. We present first the intuition behind this transformation, the formal detailed description will follow. The transformation is quite similar to the previous one; we assume again to start with a weakly-secure multi-key HA scheme MKHAut ${ }^{\prime}$ and an homomorphic trapdoor function HTDF. In addition, however, we also use two hash functions $\mathbf{H}:\{0,1\}^{*} \rightarrow\{0,1\}^{n}$ and $\mathbf{H}^{\prime}:\{0,1\}^{*} \rightarrow\{0,1\}^{n^{\prime}}$ that in the proof are modeled as random oracles. The first hash function is used to hash the dataset identifiers, i.e., to compute $H(\Delta)=\hat{\Delta}$, whereas the second one is used to obtain tags $\hat{\tau}=\mathrm{H}^{\prime}(\hat{\Delta}$, id, $\tau)$. This way the scheme MKHAut ${ }^{\prime}$ is required to support dataset identifiers that are $n$-bits strings and tags that are $n^{\prime}$-bits strings. Intuitively, modeling these hash functions as random oracles "breaks" the adaptivity of the adversary on the choice of these values. 
Our Transformation. Let $\mathrm{F}=$ (HTDF.KeyGen, $f$, Inv, HTDF.Eval ${ }^{\text {in }}$, HTDF. Eval $^{\text {out }}$ ) be an HTDF with index space $\mathcal{X}$, input space $\mathcal{U}$ (with distribution $\mathcal{D}_{\mathcal{U}}$ ), and output space $\mathcal{V}$. Let $\mathbf{H}:\{0,1\}^{*} \rightarrow\{0,1\}^{n}, \mathbf{H}^{\prime}:\{0,1\}^{*} \rightarrow\{0,1\}^{n^{\prime}}$ be two efficiently computable hash functions. Let MKHAut ${ }^{\prime}=\left(\right.$ Setup $^{\prime}$, KeyGen $^{\prime}$, Auth $^{\prime}$,

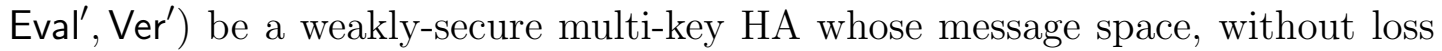
of generality, is $\mathcal{V}$. Moreover, MKHAut' has tag space $\mathcal{T}^{\prime}=\{0,1\}^{n^{\prime}}$ and it supports dataset identifiers represented as binary strings of $n$ bits. Using these tools we construct an adaptive secure homomorphic authenticator MKHAut $=$ (Setup, KeyGen, Auth, Eval, Ver) with message space $\mathcal{M}=\mathcal{X}$, and tag space $\mathcal{T}=\{0,1\}^{*}$. The scheme works as follows:

$\operatorname{Setup}\left(1^{\lambda}\right)$. Generate $\mathrm{pp}^{\prime} \leftarrow \operatorname{Setup}^{\prime}\left(1^{\lambda}\right)$ and $\left(\mathrm{pk}_{h}, \mathrm{sk}_{h}\right) \leftarrow$ HTDF.KeyGen $\left(1^{\lambda}\right)$, choose two hash functions $\mathbf{H}:\{0,1\}^{*} \rightarrow\{0,1\}^{n}, \mathbf{H}^{\prime}:\{0,1\}^{*} \rightarrow\{0,1\}^{n^{\prime}}$, and output $\mathrm{pp}=\left(\mathrm{pp}^{\prime}, \mathrm{pk}_{h}, \mathrm{H}, \mathrm{H}^{\prime}\right)$.

$\operatorname{KeyGen}(\mathrm{pp})$. Generate $\left(a k^{\prime}, \mathrm{ek}^{\prime}, \mathrm{vk}^{\prime}\right) \leftarrow \operatorname{KeyGen}\left(\mathrm{pp}^{\prime}\right)$, and output $a \mathrm{k}=\mathrm{ak}$, ek $=$ $\mathrm{ek}^{\prime}$ and $v \mathrm{k}=\mathrm{vk}^{\prime}$.

Auth(sk, $\Delta, \ell, \mathrm{m})$. Evaluate $\hat{\Delta} \leftarrow \mathrm{H}(\Delta)$ and $\hat{\tau} \leftarrow \mathrm{H}^{\prime}(\hat{\Delta}$, (id, $\left.\tau)\right)$ and set $\hat{\ell}=($ id, $\hat{\tau})$. Sample $u \stackrel{\$}{\leftarrow} \mathcal{D}_{\mathcal{U}}$, compute $v \leftarrow f_{\mathrm{pk}_{h}, \mathrm{~m}}(u)$ and finally compute $\bar{\sigma} \leftarrow \operatorname{Sign}^{\prime}\left(\mathrm{ak}^{\prime}\right.$, $\hat{\Delta}, \hat{\ell}, v)$ and output $\sigma:=(v, u, \bar{\sigma})$.

$\operatorname{Eval}\left(g,\left\{\sigma_{i}, \mathcal{K}_{i}\right\}_{i \in[t]}\right)$. This algorithm is identical to the one of the previous transformation.

$\operatorname{Ver}\left(\mathcal{P}:=\left(g, \ell_{1}, \ldots, \ell_{t}\right), \Delta,\left\{\mathrm{vk}_{\mathrm{id}}\right\}_{\in \mathcal{P}}, \mathrm{m}, \sigma\right)$. Evaluate $\hat{\Delta}=\mathrm{H}(\Delta)$ and, for all $i \in[t]$ compute $\hat{\tau}_{i}=\mathrm{H}^{\prime}\left(\hat{\Delta}, \ell_{i}\right)$ and set $\hat{\ell}_{i}=\left(\operatorname{id}_{i}, \hat{\tau}_{i}\right)$. Parse $\sigma=(v, u, \bar{\sigma})$ and output 1 if both $f_{\mathrm{pk}_{h}, \mathrm{~m}}(u)=v$ and $\operatorname{Ver}^{\prime}\left(\hat{\mathcal{P}}:=\left(g^{\prime}, \hat{\ell}_{1}, \ldots, \hat{\ell}_{t}\right), \hat{\Delta},\left\{\mathrm{vk}_{\mathrm{id}}^{\prime}\right\}_{\mathrm{id} \in \hat{\mathcal{P}}}, v, \sigma\right)=1$. Else output 0.

As for the previous transformation, the correctness of the scheme immediately follows from correctness of MKHAut', F, and the hash functions.

Theorem 20. If $\mathrm{F}$ is a secure HTDF, MKHAut' is a weakly secure multi-key homomorphic authenticator and the hash functions $\mathrm{H}$ and $\mathrm{H}^{\prime}$ are modeled as random oracles then the homomorphic authenticator MKHAut defined above is adaptively secure in the random oracle model.

Proof. The analysis of the different kinds of forgeries is exactly the same as the one presented at the beginning of the proof of Theorem 19. However, in the case of an arbitrary tag space the reduction needs to be adapted since it is not possible to make a query for every element of the tag space during the authenticationquery phase (since the space now is exponentially large), contrarily to what it did in the proof of Theorem 19 . We bypass this problem moving to the random oracle model. 
As we did for the polynomially bounded case, we show here that for every $\mathcal{A}$ producing forgeries of type 1, 2.(a) or 3 there exists an adversary that breaks the weak security of MKHAut', whereas for every $\mathcal{A}$ producing a forgery of type 2.(b) it is possible to break the security of $F$. As before, for what regards MKHAut', we show how to build a reduction $\mathcal{B}$ which uses any Type 1 or Type 2.(a) forgeries made by $\mathcal{A}$ to break MKHAut ${ }^{\prime}$ and that a similar reduction $\mathcal{B}^{\prime}$ can do the same with Type 3 forgeries.

In the following proofs, we again set $Q=\operatorname{poly}(\lambda)$ to be the total number of queries made by $\mathcal{A}$ and $Q_{\text {id }}, Q_{\mathcal{T}}, Q_{\Delta}$ to be respectively the number of distinct identities, tags and datasets which are queried by $\mathcal{A}$ during the authenticationquery phase.

Lemma 28. If $\mathcal{A}$ is an adversary that has non-negligible advantage $\epsilon$ against the security of MKHAut by producing a Type 1 (respectively Type 2.(a)) forgery, then it is possible to build an adversary $\mathcal{B}$ that has advantage $\epsilon-Q / 2^{n}$ against the weak security of MKHAut' by producing a Type 1 (respectively Type 2) forgery.

Proof. We want to build a reduction $\mathcal{B}$ which uses a Type 1 or Type 2(a) forgery by $\mathcal{A}$ against MKHAut to break the security of MKHAut'. We recall that $Q_{\text {id }}, Q_{\Delta}, Q_{\mathcal{T}}$ respectively are the number of different identities, dataset and labels which are queried by $\mathcal{A}$ during the signing-query phase. For any $(i, k) \in\left[Q_{\text {id }}\right] \times$ $\left[Q_{\Delta}\right], \mathcal{B}$ chooses random strings $\left\{\hat{\tau}_{j, k}^{i}\right\}_{j \in\left[Q_{\mathcal{T}}\right]} \stackrel{\$}{\leftarrow}\{0,1\}^{n^{\prime}}$ and sets $\mathrm{T}_{i, k}:=\left\{\hat{\tau}_{j, k}^{i}:\right.$ $\left.j \in\left[Q_{\mathcal{T}}\right]\right\}$; then, $\mathcal{B}$ samples $Q_{\text {id }} \cdot Q_{\mathcal{T}} \cdot Q_{\Delta}$ elements $\left\{\left\{v_{j, k}^{i}\right\}_{(i, j, k) \in\left[Q_{\text {id }}\right] \times\left[Q_{\mathcal{T}}\right] \times\left[Q_{\Delta}\right]} \underset{\leftarrow}{\leftarrow} \mathcal{V}\right.$. Moreover, the reduction chooses a pair of keys $\left(\mathrm{pk}_{h}, \mathrm{sk}_{h}\right)$ for $\mathrm{F}$.

Following the definition of weak-security, $\mathcal{B}$ sends $\left\{\left(v_{j, k}^{i}, \hat{\tau}_{j, k}^{i}\right)\right\}_{(i, j, k) \in\left[Q_{\text {id }}\right] \times\left[Q_{\mathcal{T}}\right] \times\left[Q_{\Delta}\right]}$ to the challenger $\mathcal{C}$ and gets back the public parameters $\mathrm{pp}^{\prime}$ of MKHAut. Next, $\mathcal{B}$ sets $\mathrm{pp}=\left(\mathrm{pp}^{\prime}, \mathrm{pk}_{h}, \mathrm{H}, \mathrm{H}^{\prime}\right)$, sends pp to $\mathcal{A}$ and initialises a counter $\mathrm{cnt}=0$. When $\mathcal{A}$ starts making its queries, $\mathcal{B}$ answers as described below.

First of all, let $\Delta_{1}, \ldots, \Delta_{Q_{\Delta}}$ be all the distinct dataset identifiers queried, in order, by $\mathcal{A}$ in authentication queries; similarly, let $\mathrm{id}_{1}, \ldots, \mathrm{id}_{Q_{\text {id }}}$ be the distinct identities queried, in order, by $\mathcal{A}$ in authentication queries too.

For hash queries to $\mathrm{H}$ or $\mathrm{H}^{\prime}, \mathcal{B}$ prepares random strings $\hat{\Delta}_{1}, \ldots, \hat{\Delta}_{Q_{\Delta}} \stackrel{\$}{\leftarrow}\{0,1\}^{n}$ and answer queries as follows.

$\mathrm{H}(\Delta)$ : If $\Delta=\Delta_{k}$, i.e., $\Delta$ is the $k$-th distinct dataset identitifier queried in authentication, then $\mathcal{B}$ answers with $\hat{\Delta}_{k}$. Otherwise, $\mathcal{B}$ samples a random $\hat{\Delta} \stackrel{\$}{\leftarrow}\{0,1\}^{n}$ and replies with $\hat{\Delta}$.

$\mathrm{H}^{\prime}(\hat{\Delta}$, id, $\tau)$ : If $\hat{\Delta}=\hat{\Delta}_{k}$ (i.e., $\hat{\Delta}$ is the $k$-th one of the $Q_{\Delta}$ random strings initially chosen) and id $=\mathrm{id}_{i}$ (i.e., id is the $i$-th distinct identity queried in authentication), then $\mathcal{B}$ increments cnt $\leftarrow$ cnt +1 and replies with $\hat{\tau}_{\mathrm{cnt}, k}^{i}$. Otherwise, if $\hat{\Delta} \notin\left\{\hat{\Delta}_{1}, \ldots, \hat{\Delta}_{Q_{\Delta}}\right\}$ or id $\notin\left\{\operatorname{id}_{1}, \ldots\right.$, id $\left._{Q_{\text {id }}}\right\}$, then $\mathcal{B}$ replies with a randomly chosen $\hat{\tau} \stackrel{\$}{\leftarrow}\{0,1\}^{n^{\prime}}$. 
We define bad' $^{\prime}$ be the event for which $\mathcal{A}$ makes a hash query $\mathrm{H}^{\prime}(\hat{\Delta}$, id, $\tau)$ such that $\hat{\Delta}=\hat{\Delta}_{k}$, for some $k$, but $\Delta_{k}$ was never returned earlier as output of $\mathrm{H}$. It is straightforward to see that $\operatorname{Pr}\left[\mathrm{bad}^{\prime}\right] \leq Q / 2^{n}$ is negligible.

When $\mathcal{A}$ makes an authentication query of the form $(\Delta, \ell:=($ id, $\tau), \mathrm{m}), \mathcal{B}$ answers as follows. First, makes the corresponding hash queries $\hat{\Delta}=\mathrm{H}(\Delta)$ and $\hat{\tau}=\mathrm{H}^{\prime}(\hat{\Delta}$, (id, $\left.\tau)\right)$, if not already done. Then, notices that it must be $\hat{\Delta}=\hat{\Delta}_{k}$ for some $k \in\left[Q_{\Delta}\right]$ and $\hat{\tau}=\hat{\tau}_{j, k}^{i}$, for some proper $, i, j$. Then $\mathcal{B}$ can proceed as in the proof of Lemma 25 to compute $u_{j, k}^{i}$ and finally return $\bar{\sigma}_{j, k}^{i}$. The remaining part of the simulation proceeds the same as in Lemma 25.

Lemma 29. If $\mathcal{A}$ is an adversary that has non-negligible advantage $\epsilon$ against the security of MKHAut by producing a Type 3 forgery, then it is possible to build an adversary $\mathcal{B}^{\prime}$ that has non-negligible advantage $\epsilon /\left(Q_{\mathrm{id}} \cdot Q_{\mathcal{T}} \cdot Q_{\Delta}\right)-Q / 2^{n}$ against the weak security of MKHAut' by producing a Type 3 forgery.

Proof. The reduction $\mathcal{B}^{\prime}$ works similarly to the one in Lemma 26 except that $\mathcal{B}^{\prime}$ also simulates hash queries as in Lemma 28.

The game starts with $\mathcal{B}^{\prime}$ sampling $Q_{\text {id }} \cdot Q_{\mathcal{T}} \cdot Q_{\Delta}$ elements $v_{j, k}^{i} \stackrel{\$}{\leftarrow} \mathcal{V}$ and strings $\hat{\tau}_{j, k}^{i} \stackrel{\$}{\leftarrow}\{0,1\}^{n^{\prime}}$ uniformly at random for all $i \in\left[Q_{\mathrm{id}}\right], j \in\left[Q_{\mathcal{T}}\right], k \in\left[Q_{\Delta}\right]$. Next $\mathcal{B}^{\prime}$ chooses a triple $(\bar{i}, \bar{j}, \bar{k}) \stackrel{\$}{\leftarrow}\left[Q_{\text {id }}\right] \times\left[Q_{\mathcal{T}}\right] \times\left[Q_{\Delta}\right]$, generates keys $\left(\mathrm{pk}_{h}, \mathrm{sk}_{h}\right)$ for the homomorphic trapdoor function $\mathrm{F}$, and sends to its challenger $\mathcal{C}$ the values $\left\{\left(v_{j, k}^{i}, \hat{\tau}_{j}\right)\right\}_{(i, j, k) \in\left\{\left[Q_{\mathrm{id}}\right] \times\left[Q_{\mathcal{T}}\right] \times\left[Q_{\Delta}\right]\right\} \backslash(\bar{i}, \bar{j}, \bar{k})}$. Basically, this is the same as in the proof of Lemma 28 except that $\mathcal{B}^{\prime}$ does not send any value related to the triple $(\bar{i}, \bar{j}, \bar{k})$. Intuitively, this can be interpreted as $\mathcal{B}^{\prime}$ betting on the dataset $\Delta_{\bar{k}}$ and the label $\ell=\left(\operatorname{id}_{i}, \tau_{j}\right)$ over which the adversary $\mathcal{A}$ will make the Type 3 forgery.

$\mathcal{B}^{\prime}$ answers the queries of $\mathcal{A}$ exactly as in Lemma 28 except if $\mathcal{A}$ makes a query $(\Delta, \ell:=(\mathrm{id}, \tau), \mathrm{m})$ such that $\mathrm{H}(\Delta)=\hat{\Delta}_{\bar{k}}$, id $=\mathrm{id}_{\bar{i}}$ and $\mathrm{H}^{\prime}\left(\hat{\Delta}_{k},(\mathrm{id}, \tau)\right)=\hat{\tau}_{\bar{j}, \bar{k}}^{\bar{i}}$. If this case occurs $\mathcal{B}^{\prime}$ aborts.

The remaining part of the simulation is basically the same as that of Lemma 26. Namely, as long as the guess of $\mathcal{B}^{\prime}$ is correct (which is the case with probability $\left.1 /\left(Q_{\text {id }} \cdot Q_{\mathcal{T}} \cdot Q_{\Delta}\right)\right)$ and there is no other bad event in the simulation of hash queries (which is the case with non-negligible probability $Q / 2^{n}$ as in Lemma 28), $\mathcal{B}^{\prime}$ can use $\mathcal{A}$ 's Type 3 forgery to output a Type 3 forgery for the weakly-secure scheme.

Lemma 30. If $\mathcal{A}$ is an adversary that has non-negligible advantage $\epsilon$ against the security of MKHAut by producing a Type 2.(b) forgery, then it is possible to build an adversary $\mathcal{B}^{\prime \prime}$ which breaks the security of the HTDF F with non-negligible probability $\epsilon$.

It is not hard to see that this case of the proof is basically identical to that in Lemma 27, and thus we omit the proof. 


\subsection{Our Multi-Key Homomorphic MAC from OWFs}

In this section, we describe our construction of a multi-key homomorphic authenticator with private verification keys and supporting the evaluation of low-degree arithmetic circuits. More precisely, for a computation represented by an arithmetic circuit of degree $d$ and involving inputs from $n$ distinct identities, the final authenticator has size $\left(\begin{array}{c}n+d \\ d\end{array}\right)$, that is bounded by $\operatorname{poly}(n)$ (for constant $d$ ) or by $\operatorname{poly}(d)$ (for constant $n$ ). Essentially, the authenticators of our scheme grow with the degree of the circuit and the number of distinct users involved in the computation, whereas their size remains independent of the total number of inputs / users. This property is particularly desirable in contexts that involve a small set of users each of which contributes with several inputs.

Although our multi-key homomorphic MAC supports less expressive computations than our homomorphic signatures of Section 4.5, the scheme comes with two main benefits. First, it is based on a simple, general assumption: it relies on pseudo-random functions and thus is secure only assuming existence of one-way functions (OWF). Second, the scheme is very intuitive and efficient: fresh MACs essentially consist only of two $\mathbb{F}_{p}$ field elements (where $p$ is a prime of $\lambda$ bits) and an identity identifier; after evaluation, the authenticators consist of $\left(\begin{array}{c}n+d \\ d\end{array}\right)$ elements in $\mathbb{F}_{p}$, and homomorphic operations are simply additions and multiplications in the multi-variate polynomial ring $\mathbb{F}_{p}\left[X_{1}, \ldots, X_{n}\right]$.

We describe the five algorithms of our scheme MKHMac below. We note that our solution is presented for single data set only. However, since it admits labels that are arbitrarily long strings it is straight-forward to extend the scheme for handling multiple data sets: simply redefine each tag $\tau$ as consisting of two substrings $\tau=\left(\Delta, \tau^{\prime}\right)$ where $\Delta$ is the dataset name and $\tau^{\prime}$ the actual tag.

Setup $\left(1^{\lambda}\right)$. The setup algorithm generates a $\lambda$-bit prime $p$ and let the message space be $\mathcal{M}:=\mathbb{F}_{p}$. The set of identities is ID $=[\mathrm{C}]$ for some integer bound $C \in \mathbb{N}$, while the tag space consists of arbitrary binary strings, i.e., $\mathcal{T}=\{0,1\}^{*}$. The set $\mathbb{F}$ of admissible functions is made up of all arithmetic circuits whose degree $d$ is bounded by some polynomial in the security parameter. The setup algorithm outputs the public parameters pp which include the descriptions of $\mathcal{M}, I D, \mathcal{T}, \mathbb{F}$ as in Section 4.4, as well as the description of a PRF family $F: \mathcal{K} \times\{0,1\}^{*} \rightarrow \mathbb{F}_{p}$ with seed space $\mathcal{K}$. The public parameters define also the authenticator space. Each authenticator $\sigma$ consists of a pair $(\mathrm{I}, \mathrm{y})$ where $\mathrm{I} \subseteq \mathrm{ID}$ and $\mathrm{y}$ is in the $\mathrm{C}$-variate polynomial ring $\mathbb{F}_{p}\left[X_{1}, \ldots, X_{\mathrm{C}}\right]$. More precisely, if $\mathrm{C}$ is set up as a very large number (e.g., $\mathrm{C}=2^{\lambda}$ ) the polynomials y can still live in some smaller sub-rings of $\mathbb{F}_{p}\left[X_{1}, \ldots, X_{\mathrm{C}}\right]$.

$\operatorname{KeyGen}(\mathrm{pp})$. The key generation algorithm picks a random $x \stackrel{\$}{\leftarrow} \mathbb{F}_{p}^{*}$, a PRF seed $K \stackrel{\$}{K}$, and outputs (ak, ek, vk) where ak $=\mathrm{vk}=(x, K)$ and ek is void. 
Auth(sk, $\ell, \mathrm{m})$. In order to authenticate the message $\mathrm{m}$ with label $\ell=($ id, $\tau) \in$ $\mathrm{ID} \times \mathcal{T}$, the authentication algorithm produces an authenticator $\sigma=(\mathrm{I}, \mathrm{y})$ where $\mathrm{I} \subseteq \mathrm{ID}$ and $\mathrm{y} \in \mathbb{F}_{p}\left[X_{\text {id }}\right] \subset \mathbb{F}_{p}\left[X_{1}, \ldots, X_{\mathrm{C}}\right]$. The set $\mathrm{I}$ is simply $\{$ id $\}$. The polynomial y is a degree- 1 polynomial in the variable $X_{\text {id }}$ such that $\mathrm{y}(0)=\mathrm{m}$ and $\mathrm{y}\left(x_{\mathrm{id}}\right)=F\left(K_{\mathrm{id}}, \ell\right)$. Note that the coefficients of $\mathrm{y}\left(X_{\mathrm{id}}\right)=$ $y_{0}+y_{\text {id }} X_{\text {id }} \in \mathbb{F}_{p}\left[X_{\text {id }}\right]$ can be efficiently computed with the knowledge of $x_{\text {id }}$ by setting $y_{0}=\mathrm{m}$ and $y_{\text {id }}=\frac{F\left(K_{\mathrm{id}}, \ell\right)-\mathrm{m}}{x_{\mathrm{id}}}$. Moreover, y can be compactly represented by only giving the coefficients $y_{0}, y_{\text {id }} \in \mathbb{F}_{p}$.

$\operatorname{Eval}\left(f,\left\{\sigma_{k}\right\}_{k \in[t]}\right)$. Given a $t$-input arithmetic circuit $f: \mathbb{F}_{p}^{t} \rightarrow \mathbb{F}_{p}$, and the $t$ authenticators $\left\{\sigma_{k}:=\left(\mathrm{I}_{k}, \mathrm{y}_{k}\right)\right\}_{k}$, the evaluation algorithm outputs $\sigma=(\mathrm{I}, \mathrm{y})$ obtained in the following way. First, it determines all the identities involved in the computation by setting $\mathbf{I}=\cup_{k=1}^{t} \mathrm{I}_{k}$. Then every polynomial $\mathrm{y}_{k}$ is "expanded" into a polynomial $\hat{y}_{k}$, defined on the variables $X_{\text {id }}$ corresponding to all the identities in $\mathrm{I}$. This is done using the canonical embedding $\mathbb{F}_{p}\left[X_{\text {id }}\right.$ : id $\left.\in \mathrm{I}_{k}\right] \hookrightarrow \mathbb{F}_{p}\left[X_{\text {id }}:\right.$ id $\left.\in \mathrm{I}\right]$. It is worth noticing that the terms of $\hat{\mathrm{y}}_{k}$ that depend on variables in $\mathrm{I} \backslash \mathrm{I}_{k}$ have coefficient 0 . Next, let $\hat{f}: \mathbb{F}_{p}\left[X_{\text {id }}:\right.$ id $\in$ I] $]^{t} \rightarrow \mathbb{F}_{p}\left[X_{\text {id }}:\right.$ id $\left.\in \mathrm{I}\right]$ be the arithmetic circuit corresponding to the given $f$, i.e., $\hat{f}$ is the same as $f$ except that additions (resp. multiplications) in $\mathbb{F}_{p}$ are replaced by additions (resp. multiplications) over the polynomial ring $\mathbb{F}_{p}\left[X_{\text {id }}:\right.$ id $\left.\in \mathrm{I}\right]$. Finally, y is obtained as $\mathrm{y}=\hat{f}\left(\hat{\mathrm{y}}_{1}, \ldots, \hat{\mathrm{y}}_{t}\right)$.

$\operatorname{Ver}\left(\mathcal{P},\left\{\mathrm{vk}_{\mathrm{id}}\right\}_{\mathrm{id} \in \mathcal{P}}, \mathrm{m}, \sigma\right)$. Let $\mathcal{P}=\left(f, \ell_{1}, \ldots, \ell_{t}\right)$ be a labeled program where $f$ is a degree- $d$ arithmetic circuit and every label is of the form $\ell_{k}=\left(\mathrm{id}_{k}, \tau_{k}\right)$. Let $\sigma=(\mathrm{I}, \mathrm{y})$ where $\mathbf{I}=\left\{\overline{\mathrm{id}}_{1}, \ldots, \overline{\mathrm{id}}_{n}\right\}$ with $\overline{\mathrm{id}}_{i} \neq \overline{\mathrm{id}}_{j}$ for $i \neq j$. The verification algorithm outputs 1 (accept) if and only if the authenticator satisfies the following three checks. Otherwise it outputs 0 (reject).

$$
\begin{aligned}
\left\{\overline{\mathrm{id}}_{1}, \ldots, \overline{\mathrm{id}}_{n}\right\} & =\{\mathrm{id}: \mathrm{id} \in \mathcal{P}\} \\
\mathrm{y}(0, \ldots, 0) & =\mathrm{m} \\
\mathrm{y}\left(x_{\mathrm{id}_{1}}, \ldots, x_{\overline{\mathrm{id}}_{n}}\right) & =f\left(F\left(K_{\mathrm{id}_{1}}, \ell_{1}\right), \ldots, F\left(K_{\mathrm{id}_{t}}, \ell_{t}\right)\right) .
\end{aligned}
$$

In the following sections, we discuss the efficiency and succinctness of our MKHMac (Section 4.6.2) and prove the correctness of our scheme (Section 4.6.3). Section 4.6.1 is devoted to the security analysis of the proposed MKHMac scheme.

\subsubsection{Polynomials Representations}

In the sequel we adopt the multi-index notation for multivariate polynomials. We consider an element $f \in \mathbb{F}_{p}\left[X_{1}, \ldots, X_{n}\right]$ as a finite sum of terms of the form $c \cdot \mathbf{X}^{\mathbf{e}}$ where $c \in \mathbb{F}_{p} \backslash\{0\}$ is a non-zero coefficient, $\mathbf{e}=\left(e_{1}, \ldots, e_{n}\right) \in \mathbb{N}^{n}$ is an exponent vector and $\mathbf{X}^{\mathrm{e}}$ is a shorthand for $X_{1}^{e_{1}} \cdots X_{n}^{e_{n}}$. 
Here we recall two main methods to represent multivariate polynomials: the completely dense representation and the sparse representation. Both representations require an ordering on the set of unknowns.

In the completely dense representation, a polynomial $f \in \mathbb{F}_{p}[\mathbf{X}]=\mathbb{F}_{p}\left[X_{1}, \ldots\right.$, $\left.X_{n}\right]$ is stored as a $\left(\prod_{i=1}^{n} d_{i}\right)$-dimensional array with entries in $\mathbb{F}_{p}$, such that the entry at index $\mathbf{e}=\left(e_{1}, \ldots, e_{n}\right)$ is the coefficient of the term $\mathbf{X}^{\mathbf{e}}$ in $f$. The completely dense representation allows constant-time access to any coefficient of $f$, and using Kronecker substitution it is possible to easily apply all "fast" methods known for dense univariate arithmetic.

The sparse representation (which corresponds to the default representation of polynomials in most computer algebra systems) of a multivariate polynomial $f \in$ $\mathbb{F}_{p}\left[X_{1}, \ldots, X_{n}\right]$ is a list of $t$ coefficient-exponent couples $\left(\left(c_{1}, \mathbf{e}_{1}\right), \ldots,\left(c_{t}, \mathbf{e}_{t}\right)\right) \in$ $\left(\mathbb{F}_{p} \times \mathbb{N}^{n}\right)^{t}$, where $t$ denotes the total number of distinct terms (monomials) in $f$. Setting $d>\operatorname{maxdeg}(f)$, the size of the sparse representation is $O(n t \log d)$. The sparse representation is the most compact polynomial representation, however, its small size entails higher operation costs. We recall the costs relevant to our scheme in Section 4.6.

\subsubsection{Efficiency and Succinctness}

Below we discuss the efficiency and the succinctness of our multi-key HA scheme. We focus first on succinctness and later we discuss the cost of running Eval on our authenticators (the cost of the other algorithms can be easily extracted from their description).

Succinctness. Let us consider the case of an authenticator $\sigma$ which was obtained after running Eval on a circuit of degree $d$ and taking inputs from $n$ distinct identities. Note that every $\sigma$ consists of two elements: a set $\mathrm{I} \subseteq[\mathrm{C}]$ and a polynomial y $\in \mathbb{F}_{p}\left[X_{\text {id }}:\right.$ id $\left.\in \mathrm{I}\right]$.

For the set I, it is easy to see that $|\mathrm{I}|=n$ and $\mathrm{I}$ can be represented with $n \log \mathrm{C}$ bits. The other part of the authenticator, $\mathrm{y}$, is instead an $n$-variate polynomial in $\mathbb{F}_{p}\left[X_{\overline{\mathrm{id}}_{1}}, \ldots, X_{\overline{\mathrm{i}}_{n}}\right]$ of degree $d$. Since the circuit degree is $d$, the maximum number of coefficients of $\mathrm{y}$ is $\left(\begin{array}{c}n+d \\ d\end{array}\right)$. More precisely, the total size of $\mathrm{y}$ depends on the particular representation of the multi-variate polynomial y which is chosen for implementation. In the Section 4.6.1 we discuss some possible representations (further details can also be found in [111]). For example, when employing the sparse representation of polynomials, the size of y is bounded by $O(n t \log d)$ where $t$ is the number of non-zero coefficients in y (note that in the worst case, a polynomial y $\in \mathbb{F}_{p}\left[X_{\overline{\mathrm{id}}_{1}}, \ldots, X_{\overline{\mathrm{i}}_{n}}\right]$ of degree $d$ has at most $t=\left(\begin{array}{c}n+d \\ d\end{array}\right)$ non-zero coefficients). Thus, setting $\log \mathrm{C} \approx \log p \approx \lambda$, we have that the size in bits of the authenticator $\sigma$ is $|\sigma| \leq \lambda n+\lambda\left(\begin{array}{c}n+d \\ d\end{array}\right)$. Ignoring the security parameter, we have that $|\sigma|=\operatorname{poly}(n)$ when $d$ is constant, or $|\sigma|=\operatorname{poly}(d)$ when $n$ is constant.

Efficiency of Eval. In what follows, we discuss the cost of computing ad- 
ditions and multiplications over authenticators in our MKHMac scheme. Let $\sigma^{(i)}=\left(\mathrm{I}^{(i)}, \mathbf{y}^{(i)}\right)$, for $i=1,2$ be two authenticators and consider the operation $\sigma=\operatorname{Eval}\left(g, \sigma^{(1)}, \sigma^{(2)}\right)$ where $g$ is a fan-in-2 addition or multiplication gate. In both cases the set $\mathbf{I}$ of identities of $\sigma=(\mathbf{I}, \mathbf{y})$ is obtained as the union $\mathbf{I}=\mathbf{I}^{(1)} \cup \mathbf{I}^{(2)}$ that can be computed in time $O(n)$, where $n=|\boldsymbol{I}|$, assuming the sets $\mathbf{I}^{(1)}, \mathbf{I}^{(2)}$ are ordered. Regarding the computation of $y$ from $\mathrm{y}^{(1)}$ and $\mathrm{y}^{(2)}$, one has to first embed each $\mathrm{y}_{i}$ into the ring $\mathbb{F}_{p}\left[X_{\overline{\text { id }}}:\right.$ id $\left.\in \mathrm{I}\right]$, and then evaluate addition (resp. multiplication) over $\mathbb{F}_{p}\left[X_{\overline{\text { id }}}: \overline{i d} \in \mathrm{I}\right]$. Again, the costs of these operations depend on the adopted representation [111, 66].

Using the sparse representation of polynomials, expanding a y having $t$ nonzero coefficients into an $n$-variate polynomial y requires time at most $O(t n)$. To give an idea, such expansion indeed consists simply into inserting zeros in the correct positions of the exponent vectors of every non-zero monomial term of $y$. On the other hand, the complexity of operations (additions and multiplications) on polynomials using the sparse representation is usually estimated in terms of the number of monomial comparisons. The cost of such comparisons depends on the specific monomial ordering chosen, but is usually $O(n \log d)$, where $n$ is the total number of variables and $d$ is the maximum degree. Given two polynomials in sparse representation having $t_{1}$ and $t_{2}$ non-zero terms respectively, addition costs about $O\left(t_{1} t_{2}\right)$ monomial comparisons (if the monomial terms are stored in sorted order the cost of addition drops to $O\left(t_{1}+t_{2}\right)$ ), while multiplication requires to add (merge) $t_{2}$ intermediate products of $t_{1}$ terms each, and can be performed with $O\left(t_{1} t_{2} \log t_{2}\right)$ monomial comparisons [66].

\subsubsection{Correctness}

In what follows we discuss the correctness of the proposed MKHMac.

By construction, each fresh authenticator $\sigma=(\mathrm{I}, \mathrm{y})$ of a message $\mathrm{m}$ labeled by $\ell:=(\mathrm{id}, \tau)$ is of the form $\mathrm{I}=\{\mathrm{id}\}$ and $\mathrm{y}\left(X_{\mathrm{id}}\right):=y_{0}+y_{\mathrm{id}} X_{\mathrm{id}}=\mathrm{m}+\frac{F\left(K_{\mathrm{id}}, \ell\right)-\mathrm{m}}{x_{\mathrm{id}}} X_{\mathrm{id}}$. Thus the set I satisfies equation (4.5) since $\left\{\right.$ id : id $\left.\in \mathcal{I}_{\ell}\right\}=\{$ id $\}$. The two last verification checks (4.6) and (4.7) are automatically granted for the identity program $\mathcal{I}_{\ell}$ because $\mathrm{y}(0)=y_{0}=\mathrm{m}$ and $\mathrm{y}\left(x_{\mathrm{id}}\right)=\mathrm{m}+\frac{F\left(K_{\mathrm{id}}, \ell\right)-\mathrm{m}}{x_{\text {id }}} x_{\text {id }}=F\left(K_{\text {id }}, \ell\right)$.

Evaluation Correctness. The correctness of the Eval algorithm essentially comes from the structure of the multi-variate polynomial ring. Nevertheless, we will prove the evaluation correctness property for generic fan-in-2 addition and multiplication gates (by associativity, this is the inductive step to prove evaluation correctness for any circuit $f$ ).

For $i=1,2$, let $\left(\mathcal{P}_{i}, \mathrm{~m}_{i}, \sigma^{(i)}\right)$ be a triple such that $\operatorname{Ver}\left(\mathcal{P}_{i},\left\{\mathrm{vk}_{\mathrm{id}}\right\}_{\mathrm{id} \in \mathcal{P}_{i}}, \mathrm{~m}_{i}, \sigma^{(i)}\right)=$ 1 , where $\mathcal{P}_{i}=\left(f_{i}, \ell_{1}^{(i)}, \ldots, \ell_{t_{i}}^{(i)}\right)$. We need to show that for every fan-in-2 gate $g$ we have $\operatorname{Ver}\left(\mathcal{P},\left\{\mathrm{vk}_{\mathrm{id}}\right\}_{\text {id } \in \mathcal{P}}, \mathrm{m}, \sigma\right)=1$ where $\mathcal{P}=g\left(\mathcal{P}_{1}, \mathcal{P}_{2}\right), \mathrm{m}=g\left(\mathrm{~m}_{1}, \mathrm{~m}_{2}\right)$ and $\sigma=\operatorname{Eval}\left(g, \sigma^{(1)}, \sigma^{(2)}\right)$.

To see this, let us further expand on the meaning of assuming that $\operatorname{Ver}\left(\mathcal{P}_{i}\right.$, 
$\left.\left\{\mathrm{vk}_{\mathrm{id}}\right\}_{\mathrm{id} \in \mathcal{P}_{i}}, \mathrm{~m}_{i}, \sigma^{(i)}\right)=1$. For every $i=1,2$ let $\mathrm{I}^{(i)}=\left\{\overline{\mathrm{id}}_{1}^{(i)}, \ldots, \overline{\mathrm{id}}_{n_{i}}^{(i)}\right\}$ where all identities are distinct. Then verification means that the following equations hold:

$$
\begin{aligned}
\left\{\overline{\mathrm{id}}_{1}^{(i)}, \ldots, \overline{\mathrm{d}}_{n_{i}}^{(i)}\right\} & =\left\{\mathrm{id}: \mathrm{id} \in \mathcal{P}_{i}\right\} \\
\mathrm{y}^{(i)}(0, \ldots, 0) & =\mathrm{m}_{i} \\
\mathrm{y}^{(i)}\left(\overline{\mathrm{id}}_{1}^{(i)}, \ldots, \overline{\mathrm{id}}_{n_{i}}^{(i)}\right) & =f_{i}\left(F\left(K_{\mathrm{id}_{1}^{(i)}}, \ell_{1}^{(i)}\right), \ldots, F\left(K_{\mathrm{id}_{t_{i}}^{(i)}}, \ell_{t_{i}}^{(i)}\right)\right)
\end{aligned}
$$

Let $\mathbf{I}=\mathbf{I}^{(1)} \cup \mathbf{I}^{(2)}$, it is trivial to check that equation 4.5) of the verification holds for $\mathrm{I}$ and $\mathcal{P}=g\left(\mathcal{P}_{1}, \mathcal{P}_{2}\right)$ since

$$
\mathrm{I}=\mathrm{I}^{(1)} \cup \mathrm{I}^{(2)}=\left\{\text { id }: \text { id } \in \mathcal{P}_{1}\right\} \cup\left\{\text { id }: \text { id } \in \mathcal{P}_{1}\right\}=\{\text { id }: \text { id } \in \mathcal{P}\},
$$

where the second equality derives from 4.8).

Let $\mathbf{I}=\left\{\overline{\mathrm{id}}_{1}, \ldots, \overline{\mathrm{id}}_{n}\right\}$, denote by $\hat{\mathrm{y}}^{(i)}$ the $n$-variate polynomial obtained from $\mathrm{y}^{(i)}$ after the expansion process described in Eval. We need to prove that if equations (4.6) and (4.7) hold true for $\mathrm{y}^{(i)}$ they also for its expansion $\hat{\mathrm{y}}^{(i)}$ (with respect to the same labeled program $\mathcal{P}_{i}$ ). Equation $(4.9)$ and the fact that the expansion process does not affect the constant term of the polynomial trivially imply that $\hat{\mathrm{y}}^{(i)}(0)=\mathrm{m}_{i}$; whereas $\hat{\mathrm{y}}^{(i)}\left(x_{\overline{\mathrm{id}}_{1}^{(i)}}, \ldots, x_{\overline{\mathrm{id}}_{n_{i}}^{(i)}}\right)=\mathrm{y}\left(x_{\overline{\mathrm{id}}^{(i)}}, \mathrm{id} \in \mathrm{I}^{(i)}\right)=f_{i}\left(F\left(K_{\mathrm{id}_{1}^{(i)}}, \ell_{1}^{(i)}\right)\right.$, $\left.\ldots, F\left(K_{\mathrm{id}_{t_{i}}^{(i)}}, \ell_{t_{i}}^{(i)}\right)\right)$ holds because the polynomial expansion in Eval sets to 0 the coefficients of all terms that depend on the new variables.

Consider the $n$-variate polynomial y $=g\left(\hat{\mathbf{y}}^{(1)}, \hat{\mathbf{y}}^{(2)}\right)$, where $g$ is a generic gate. It is left to show that that y verifies (4.6) and (4.7). In what follows we prove correctness for a fan-in-2 addition gate; the case of a fan-in-2 multiplication gate is analogous to this one. Check (4.6) is straightforward since $\mathrm{y}(0, \ldots, 0)=\hat{y}^{(1)}(0, \ldots, 0)+\hat{y}^{(2)}(0, \ldots, 0)=\mathrm{m}_{1}+\mathrm{m}_{2}=\mathrm{m}$ holds because of equation 4.9 and the property of addition over the polynomial ring $\mathbb{F}_{p}\left[X_{\text {id }}:\right.$ id $\left.\in \mathrm{I}\right]$. For check (4.7) we have:

$\mathrm{y}\left(x_{\overline{\mathrm{i}}_{1}}, \ldots, x_{\overline{\mathrm{id}}_{n}}\right)=\hat{\mathrm{y}}^{(1)}\left(x_{\overline{\mathrm{i}}_{1}^{(1)}}, \ldots, x_{\overline{\mathrm{id}}_{n_{1}}^{(1)}}\right)+\hat{\mathrm{y}}^{(2)}\left(x_{\overline{\mathrm{i}}_{1}^{(2)}}, \ldots, x_{\overline{\mathrm{id}}_{n_{2}}^{(2)}}\right)=$ $=\mathrm{y}^{(1)}\left(x_{\overline{\mathrm{id}}_{1}^{(1)}}^{(1)}, \ldots, x_{\overline{\mathrm{id}}_{n_{1}}^{(1)}}^{(1)}\right)+\mathrm{y}^{(2)}\left(x_{\overline{\mathrm{id}}_{1}^{(2)}}^{(2)}, \ldots, x_{\overline{\mathrm{id}}_{n_{2}}^{(2)}}^{(2)}\right)=$

$=f_{1}\left(F\left(K_{\mathrm{id}_{1}^{(1)}}, \ell_{1}^{(1)}\right), \ldots, F\left(K_{\mathrm{id}_{t_{1}}^{(1)}}, \ell_{t_{1}}^{(1)}\right)\right)+f_{2}\left(F\left(K_{\mathrm{id}_{1}^{(2)}}, \ell_{1}^{(2)}\right), \ldots, F\left(K_{\mathrm{id}_{t_{2}}^{(2)}}, \ell_{t_{2}}^{(2)}\right)\right)=$ $=f\left(F\left(K_{\mathrm{id}_{1}}, \ell_{1}\right), \ldots, F\left(K_{\mathrm{id}_{t}}, \ell_{t}\right)\right)$.

where the first equality follows by construction of $y$ and by the property of addition over $\mathbb{F}_{p}\left[X_{\text {id }}:\right.$ id $\left.\in \mathrm{ID}\right]$. The second equality is derived by the definition of polynomial expansion. The third equality follows immediately from equation (4.10), while the last one holds by the composition property of labeled programs, i.e., $\mathcal{P}=g\left(\mathcal{P}_{1}, \mathcal{P}_{2}\right)$.

Security. In what follows we prove the security of our scheme against adversaries that make static corruptions, and produce forgeries according to the following restrictions. 
Definition 23 (Weak Forgery). Consider an execution of the experiment described in Section 4.4. HomUF-CMA ${ }_{\mathcal{A} \text {,MKHAut }}(\lambda)$ where $\left(\mathcal{P}^{*}, \Delta^{*}, \mathrm{~m}^{*}, \sigma^{*}\right)$ is the tuple returned by the adversary at the end of the experiment, with $\mathcal{P}^{*}=\left(f^{*}, \ell_{1}^{*}, \ldots, \ell_{t}^{*}\right)$, $\Delta^{*}$ a dataset identifier, $\mathrm{m}^{*} \in \mathcal{M}$ and $\sigma^{*}$ an authenticator. First, we say that the labeled program $\mathcal{P}^{*}$ is well-defined on a list $L$ if either one of the following two cases occurs:

1 There exists $i \in[t]$ such that $\left(\ell_{i}^{*}, \cdot\right) \notin L$ (i.e., $\mathcal{A}$ never made a query with label $\left.\ell_{i}^{*}\right)$, and $f^{*}\left(\left\{\mathrm{~m}_{j}\right\}_{\left(\ell_{j}, \mathrm{~m}_{j}\right) \in L} \cup\left\{\tilde{\mathrm{m}}_{j}\right\}_{\left(\ell_{j},\right) \notin L}\right)$ outputs the same value for all possible choices of $\tilde{\mathrm{m}}_{j} \in \mathcal{M}$;

$2 L$ contains the tuples $\left(\ell_{1}^{*}, \mathrm{~m}_{1}\right), \ldots,\left(\ell_{t}^{*}, \mathrm{~m}_{t}\right)$, for some messages $\mathrm{m}_{1}, \ldots, \mathrm{m}_{t}$.

Then we say that $\left(\mathcal{P}^{*}, \Delta^{*}, \mathrm{~m}^{*}, \sigma^{*}\right)$ is a weak forgery if $\operatorname{Ver}\left(\mathcal{P}^{*}, \Delta^{*},\left\{\mathrm{v} \mathrm{k}_{\mathrm{id}}\right\}_{\mathrm{id} \in \mathcal{P}^{*}}\right.$, $\left.\mathrm{m}^{*}, \sigma^{*}\right)=1$ and either one of the following conditions is satisfied:

Type 1: $L_{\Delta^{*}}$ was not initialized during the game (i.e., $\Delta^{*}$ was never queried).

Type 2: $\mathcal{P}^{*}$ is well-defined on $L_{\Delta^{*}}$ but $\mathrm{m}^{*} \neq f^{*}\left(\left\{\mathrm{~m}_{j}\right\}_{\left(\ell_{j}, \mathrm{~m}_{j}\right) \in L_{\Delta^{*}}} \cup\{0\}_{\ell_{j} \notin L_{\Delta^{*}}}\right)$ (i.e., $\mathrm{m}^{*}$ is not the correct output of $\mathcal{P}^{*}$ when executed over previously authenticated messages).

Type 3: $\mathcal{P}^{*}$ is not well-defined on $L_{\Delta^{*}}$.

Although Definition 23 is weaker than our Definition 18, we stress that the above definition still protects the verifier from adversaries that try to cheat on the output of a computation. In more details, the difference between Definition 23 and Definition 18 is the following: if $f^{*}$ has an input wire that has never been authenticated during the game (a Type 3 forgery in Definition 18), but $f^{*}$ is constant with respect to such input wire, then the above definition does not consider it a forgery. The intuitive reason why such a relaxed definition still makes sense is that "irrelevant" inputs would not help in any case the adversary to cheat on the output of $f^{*}$. Definition 23 is essentially the multi-key version of the forgery definition used in previous (single-key) homomorphic MAC works, e.g., [43]. As discussed in [63] testing whether a program is well-defined may not be doable in polynomial time in the most general case (i.e., every class of functions). However, in [44] it is shown how this can be done efficiently via a probabilistic test in the case of arithmetic circuits of degree $d$ over a finite field of order $p$ such that $d / p<1 / 2$. Finally, we notice that for our MKHMac Type 1 forgeries cannot occur as the scheme described here supports only one dataset 11

Theorem 21. If $F$ is a pseudo-random function then the multi-key homomorphic MAC described in Section 4.6 is secure against adversaries that make static corruptions of keys and produce forgeries as in Definition 23.

\footnotetext{
${ }^{11}$ As noted at the beginning of the section the extension to multiple datasets is straightforward given that tags are arbitrary strings. When such extension is applied it is easy to see that Type 1 forgeries are Type 3 ones in the underlying scheme.
} 
Note that we can deal with corruptions via our generic result of Proposition 2 . Therefore, it is sufficient to prove the security against adversaries that make no corruptions. The proof is done via a chain of games following this (intuitive) path. First, we rule out adversaries that make Type 3 forgeries. Intuitively, this can be done as the adversary has never seen one of the inputs of the computation, and in particular an input which can change the result. Second, we replace every PRF instance with a truly random function. Note that at this point the security of the scheme is information theoretic. Third, we change the way to answer verification queries that are candidates to be Type 2 forgeries. Finally, we observe that in this last game the adversary gains no information on the secret keys $x_{i}$ and thus has negligible probability of making a Type 2 forgery. A more formal description follows.

Let Game $\mathbf{0}$ be the security game between the adversary $\mathcal{A}$ and the challenger $\mathcal{C}$ described in Section 4.4, where forgeries follow the Definition 23 given above, and there are no corruptions. We define the following chain of hybrid games:

Game 1 This game proceeds as Game 0 except that the challenger answers with 0 (reject) all those verification queries $(\mathcal{P}, \mathrm{m}, \mathrm{y})$ that constitute a Type 3 forgery, i.e., such that they verify correctly and $\mathcal{P}$ is not well-defined on the list $L$.

Game 2 This is the same as Game 1 except that every PRF instance $F\left(K_{\text {id }}, \cdot\right)$ is replaced with a truly random function $\mathcal{R}_{\text {id }}:\{0,1\}^{*} \rightarrow \mathbb{F}_{p}$. Basically, for every label $\ell=($ id, $\tau)$ used in the game, while in Game 0 and Game 1 one generates the value $r_{\ell}$ as $r_{\ell}=F\left(K_{\text {id }}, \ell\right)$, in Game 2 the value $r_{\ell}$ is taken uniformly at random in $\mathbb{F}_{p}$.

Game 3 This is the same as Game 2 except that the challenger proceeds differently when answering those verification queries $(\mathcal{P}, \mathrm{m}, \sigma)$ such that $\mathcal{P}=$ $\left(f, \ell_{1}, \ldots, \ell_{t}\right)$ is well-defined on $L$. If one equation between (4.5) and (4.6) of the verification algorithm is not satisfied the challenger immediately answers with 0 (reject), without executing the check of equation (4.7). Otherwise, if both equations (4.5) and (4.6) are satisfied, $\mathcal{C}$ proceeds as follows. For every $j \in[t]$ such that $\left(\ell_{j}, \cdot\right) \notin L, \mathcal{C}$ generates a "dummy" polynomial $\mathrm{y}_{j} \in \mathbb{F}_{p}\left[X_{\text {id }}:\right.$ id $\left.\in \ell_{j}\right]$ (e.g., taking its two coefficients randomly), whereas for all $i \in[t]$ such that $\left(\ell_{i}, \mathrm{~m}_{i}\right) \in L, \mathcal{C}$ fetches the authenticators $\mathrm{y}_{i}$ generated previously during the authentication queries phase of the game. The challenger then computes $\operatorname{Eval}\left(f, \sigma_{1}, \ldots, \sigma_{t}\right)$ to obtain the output $\hat{\sigma}=(\hat{\mathrm{I}}, \hat{\mathrm{y}})$, and answers the verification query as follows:

- If $\mathrm{y}=\hat{\mathrm{y}}$ (over $\left.\mathbb{F}_{p}\left[X_{\overline{\text { id }}}: \overline{\mathrm{id}} \in \mathrm{I}=\hat{\mathrm{l}}\right]\right)$, output 1 (accept);

- If $\mathrm{y} \neq \hat{\mathrm{y}}$, compute the polynomial $z=\mathrm{y}-\hat{\mathrm{y}} \in \mathbb{F}_{p}\left[X_{\overline{\mathrm{id}}}: \overline{\mathrm{d}} \in \mathrm{I}\right]$ and output 1 (accept) if $z\left(x_{\overline{\mathrm{id}}_{1}}, \ldots, x_{\overline{\mathrm{id}}_{n}}\right)=0 \bmod p$, and 0 (reject) otherwise. 
Note that rejecting immediately queries that do not satisfy equations (4.5) or 4.6 does not change the outcome of the verification queries, and thus does not affect the distribution of the game. This change is introduced only to ease the security analysis of the game. Intuitively, this kind of queries can be immediately rejected without using any secret; in other words, the outcome of these queries reveals zero information about the secret keys. Also the second change does not affect the distribution of Game 3 compared to Game 2. This is slightly trickier to observe and is addressed in Lemma 33 .

Let $\mathrm{G}_{i}(\mathcal{A})$ denote the event that Game $i$, executed with adversary $\mathcal{A}$, outputs 1 . The proof of Theorem 21 is obtained by showing that any PPT adversary has negligible probability of distinguishing between every consecutive pair of games, as well as it has negligible probability of winning in Game 3. This is proven in the following lemmas:

Lemma 31. For every PPT $\mathcal{A}$ there is a PPT B such that $\left|\operatorname{Pr}\left[\mathrm{G}_{0}(\mathcal{A})\right]-\operatorname{Pr}\left[\mathrm{G}_{1}(\mathcal{A})\right]\right|$ $\leq Q_{\mathrm{Ver}}\left(\operatorname{Pr}\left[\mathrm{G}_{1}(\mathcal{B})\right]+d / p\right)$ where $Q_{\mathrm{Ver}}$ is the number of verification queries made by $\mathcal{A}$.

Proof. The only case in which Game 0 and Game 1 differ is when the adversary $\mathcal{A}$ makes a verification query that is a valid Type 3 forgery. Let us call this event Bad, and note that $\left|\operatorname{Pr}\left[\mathrm{G}_{0}(\mathcal{A})\right]-\operatorname{Pr}\left[\mathrm{G}_{1}(\mathcal{A})\right]\right|=\operatorname{Pr}\left[\mathrm{G}_{0}(\mathcal{A}) \wedge\right.$ Bad $]$. In what follows we bound this probability by showing that for every adversary $\mathcal{A}$ that wins in Game 0 by returning a Type 3 forgery there is an adversary $\mathcal{B}$ that, in the same game, produces a Type 2 forgery with all but negligible probability. The proof is an adaptation to the multi-key setting of Proposition 2 in [44]. For completeness we give the proof.

For every $\mathcal{A}$ we construct $\mathcal{B}$ as follows. First of all, $\mathcal{B}$ chooses randomly an index $j^{*} \stackrel{\mathbb{\$}}{\leftarrow}\left[Q_{\text {Ver }}\right]$ as a guess that $\mathcal{A}$ will make a Type 3 forgery in the $j^{*}$-th verification query. Next, $\mathcal{B}$ runs $\mathcal{A}$ and answers its queries as follows: $\mathcal{B}$ forwards to its challenger all the authentication queries of $\mathcal{A}$, and all verification queries $(\mathcal{P}, \mathrm{m}, \sigma)$ such that $\mathcal{P}$ is well-defined on the list $L$. The $j$-th verification query where $\mathcal{P}$ is not well-defined is answered with 0 (reject) if $j \neq j^{*}$. Otherwise, if $j=j^{*} \mathcal{B}$ does the following. Parse $\mathcal{P}=\left(f, \ell_{1}, \ldots, \ell_{t}\right)$; for all $i \in[t]$ such that $\left(\ell_{i}, \mathrm{~m}_{i}\right) \in L, \mathcal{B}$ fetches the message $\mathrm{m}_{i}$ from the list and set $\tilde{\mathrm{m}}_{i}=\mathrm{m}_{i}$, and for all $i \in[t]$ such that $\left(\ell_{i}, \cdot\right) \notin L, \mathcal{B}$ chooses random messages $\tilde{\mathrm{m}}_{i} \stackrel{\$}{\leftarrow} \mathbb{F}_{p}$. Then $\mathcal{B}$ computes $\tilde{\mathrm{m}}=f\left(\tilde{\mathrm{m}}_{1}, \ldots, \tilde{\mathrm{m}}_{t}\right)$ and tests if $\tilde{\mathrm{m}}=\mathrm{m}$. If this is the case then $\mathcal{B}$ aborts. Otherwise, for all $i \in[t]$ such that $\left(\ell_{i}, \cdot\right) \notin L, \mathcal{B}$ queries $\left(\ell_{i}, \tilde{\mathrm{m}}_{i}\right)$ to its challenger, and finally returns $(\mathcal{P}, \mathrm{m}, \sigma)$ as its forgery.

To complete the proof, we first observe that $\operatorname{Pr}[\tilde{\mathrm{m}}=\mathrm{m}]=d / p$ over the random choices of $\tilde{\mathrm{m}}_{i} \stackrel{\$}{\leftarrow} \mathbb{F}_{p}$ by the Schwartz-Zippel lemma [106, 118], since $f$ is not well defined and assuming that $d$ is a bound on $f$ 's degree. Then since $p$ is taken $>2^{\lambda}$ and $d=\operatorname{poly}(\lambda)$ it holds that $d / p$ is negligible. Finally, if $\mathcal{B}$ does not abort, 
$\mathcal{A}$ returns a Type 3 forgery and the guess on $j^{*}$ is correct, it is easy to see that the forgery returned by $\mathcal{B}$ is of Type 2 , i.e, $\mathcal{B}$ would win in Game 1 . Therefore $\operatorname{Pr}\left[\mathrm{G}_{1}(\mathcal{B})\right]>\operatorname{Pr}\left[\mathrm{G}_{0}(\mathcal{A}) \wedge \mathrm{Bad}\right] / Q_{\text {Ver }}-d / p$, which concludes the proof.

Lemma 32. For every PPT $\mathcal{A}$ there is a PPT D such that $\left|\operatorname{Pr}\left[\mathrm{G}_{1}(\mathcal{A})\right]-\operatorname{Pr}\left[\mathrm{G}_{2}(\mathcal{A})\right]\right|$ $\leq Q_{\mathrm{id}} \cdot \operatorname{Adv}_{F, \mathcal{D}}^{P R F}(\lambda)$, where $Q_{\text {id }}$ is the number of distinct identities queried by $\mathcal{A}$ through both authentication and verification queries.

The proof is rather simple and we only give an intuition on how it works. Given that the difference between Game 1 and Game 2 is solely in the generation of the values $r_{\ell}$; the probability of $\mathcal{A}$ winning is the same in the two games, a part from an additive factor that comes from distinguishing the PRF instance $F\left(K_{\text {id }}, \cdot\right)$ from a truly random function. Since there are at most $Q_{\text {id }}$ distinct PRF instances that are used in the game, by a fairly standard hybrid argument we have that the difference between the two games is bound by $Q_{\text {id }} \cdot \mathbf{A d v}_{F, \mathcal{D}}^{P R F}(\lambda)$ factor.

Lemma 33. For every PPT $\mathcal{A}$ we have $\operatorname{Pr}\left[\mathrm{G}_{2}(\mathcal{A})\right]=\operatorname{Pr}\left[\mathrm{G}_{3}(\mathcal{A})\right]$.

Proof. To prove the lemma we show that the outcome of every verification query made by $\mathcal{A}$ is the same in both Game 2 and Game 3. Let us consider only those queries for which the change mentioned in Game 3 applies, i.e., those in which $\mathcal{P}$ is well-defined on $L$.

As already pointed out in the description of Game 3, rejecting immediately queries that do not satisfy equations (4.5) or 4.6) does not introduce any change to the outcome of the game.

As before, let $\mathbf{I}=\left\{\overline{\mathrm{id}}_{1}, \ldots, \overline{\mathrm{id}}_{n}\right\}$ and $\hat{\mathrm{I}}=\left\{\hat{\mathrm{id}}_{1}, \ldots, \hat{\mathrm{id}}_{\hat{n}}\right\}$. Consider queries that satisfy equations (4.5) and (4.6). By correctness of the scheme, the authenticating polynomial $\hat{y}$ computed by $\mathcal{C}$ verifies correctly for $\mathcal{P}$. This in particular means that the following equation holds $\hat{y}\left(x_{\hat{\mathrm{id}}_{1}}, \ldots, x_{\hat{\mathrm{id}}_{\hat{n}}}\right)=f\left(F\left(K_{\mathrm{id}_{1}}, \ell_{1}\right), \ldots\right.$, $\left.F\left(K_{\mathrm{id}_{t}}, \ell_{t}\right)\right)$. Since checking equation 4.7 for y means checking y $\left(x_{\overline{\mathrm{i}}_{1}}, \ldots, x_{\overline{\mathrm{id}}_{n}}\right)=$ $f\left(F\left(K_{\mathrm{id}_{1}}, \ell_{1}\right), \ldots, F\left(K_{\mathrm{id}_{t}}, \ell_{t}\right)\right)$ and by hypothesis y satisfies 4.5$)$, i.e. $\hat{\mathbf{I}}=\mathrm{I}$, it is easy to see that $z\left(x_{\overline{\mathrm{id}}_{1}}, \ldots, x_{\overline{\mathrm{id}}_{n}}\right)=0$ is an equivalent check as $z=\mathrm{y}-\hat{\mathrm{y}}$.

Lemma 34. For every PPT $\mathcal{A}$ we have $\operatorname{Pr}\left[\mathrm{G}_{3}(\mathcal{A})\right] \leq Q_{\mathrm{Ver}} \cdot \frac{d}{p}$, where $Q_{\mathrm{Ver}}$ is the number of verification queries made by $\mathcal{A}$ and $d$ the (maximal) degree of the arithmetic circuits queried by $\mathcal{A}$ in verification.

Proof. In order to prove Lemma 34 we define a sequence of hybrid games departing from Game 3 , that we call Game $(3,0), \ldots$, Game $\left(3, Q_{\text {ver }}\right)$, where $Q_{\text {ver }}$ denotes the total number of verification queries performed by the adversary. For $i=0$ to $Q_{\text {Ver }}$, define Game $(3, i)$ as Game 3 except that the first $i$ verification queries that are candidate to be Type 2 forgeries (i.e., they satisfy all Type 2 requirements except that verification accepts) are answered with 0 , whereas the 
remaining $\left(Q_{\text {Ver }}-i\right)$ queries are answered as stated in Game 3. Clearly, Game $(3,0)$ is identical to Game 3, i.e., $\operatorname{Pr}\left[\mathrm{G}_{3}(\mathcal{A})\right]=\operatorname{Pr}\left[\mathrm{G}_{(3,0)}(\mathcal{A})\right]$, while

$$
\operatorname{Pr}\left[G_{\left(3, Q_{\text {Ver }}\right)}(\mathcal{A})\right]=0
$$

since in Game $\left(3, Q_{\text {Ver }}\right)$ the challenger replies 0 to any verification query.

To proceed with the proof we analyze the difference between each consecutive pair of games. For every $i \in\left[Q_{\mathrm{Ver}}\right]$, the only case in which Game $(3, i)$ may differ from Game $(3, i-1)$ is in the $i$-th verification query. Let Bad be the event in which the $i$-th verification query submitted by $\mathcal{A}$ is a valid Type 2 forgery; then $\left|\operatorname{Pr}\left[G_{(3, i-1)}(\mathcal{A})\right]-\operatorname{Pr}\left[G_{(3, i)}(\mathcal{A})\right]\right| \leq \operatorname{Pr}[\operatorname{Bad}]$. In what follows we bound the probability that Bad happens.

Notice that Bad occurs in those verification queries $(\mathcal{P}, \mathrm{m}, \sigma)$ with $\sigma=(\mathrm{I}, \mathrm{y})$ that satisfy the Type 2 requirements, pass the verification equations 4.5) and (4.6), and for which (a) y $\neq \hat{\mathrm{y}}$ and (b) $z\left(x_{\overline{\mathrm{id}}_{1}}, \ldots, x_{\overline{\mathrm{id}}_{n}}\right)=\mathrm{y}\left(x_{\overline{\mathrm{id}}_{1}}, \ldots, x_{\overline{\mathrm{id}}_{n}}\right)-$ $\hat{\mathrm{y}}\left(x_{\mathrm{id}_{1}}, \ldots, x_{\overline{\mathrm{id}}_{n}}\right)=0 \bmod p$, where $\mathbf{I}=\hat{\mathbf{I}}=\left\{\overline{\mathrm{id}}_{1}, \ldots, \overline{\mathrm{id}}_{n}\right\}$. It is easy to see that condition (a) is immediately implied by the fact that the query is a Type 2 candidate. Indeed, if $\mathrm{y}=\hat{\mathrm{y}}$ then the query would be accepted and could not be a Type 2 forgery. To see this, let $\hat{\mathrm{m}}=f\left(\left\{\mathrm{~m}_{j}\right\}_{\left(\ell_{j}, \mathrm{~m}_{j}\right) \in L_{\Delta}} \cup\{0\}_{\ell_{j} \notin L_{\Delta^{*}}}\right)$. By definition of Type 2 forgery $\mathrm{m} \neq \hat{\mathrm{m}}$ and by correctness of the scheme $\hat{y}_{0}=\hat{\mathrm{m}}$. Hence, combining the previous two equations with (4.6) we get $y_{0}=\mathbf{m} \neq \hat{\mathbf{m}}=\hat{y}_{0}$ which implies $\mathrm{y} \neq \hat{\mathrm{y}}$.

Now, we claim that in an execution of Game $(3, i), \operatorname{Pr}[\operatorname{Bad}]=\operatorname{Pr}\left[z\left(x_{\overline{\mathrm{id}}_{1}}, \ldots\right.\right.$, $\left.\left.x_{\overline{\mathrm{id}}_{n}}\right)=0\right] \leq \frac{d}{p}$.

If the values $x_{\text {id }}$ were randomly selected in $\mathbb{F}_{p}$ at the moment of Bad, the bound follows immediately from the Schwartz-Zippel Lemma [106, 118], using that $z$ is a non-zero polynomial of degree at most $d$. It remains to show that the $x_{\mathrm{id}_{j}}$ 's can indeed be selected at the moment of Bad, and to do this we show that prior to the happening of event Bad the adversary has no information about $x_{\text {id }}$, id $\in \mathrm{I}$. First, we observe that every authentication query $(\ell=(\mathrm{id}, \tau), \mathrm{m})$ does not reveal information about $x_{\text {id }}$ : the only value of the authenticator $\sigma$ which depends on $x_{\text {id }}$ is the degree- 1 coefficient of the authenticator $\mathrm{y}$ given in reply to the query; however this coefficient is uniformly distributed in $\mathbb{F}_{p}$ due to randomness of $r_{\ell}$. Second, all the previous $i-1$ verification queries in Game $(3, i)$ are answered without the use of any secret $x_{\text {id }}$, id $\in[\mathrm{C}]$ : they are either answered without checking condition (b), or are directly rejected (without evaluating (b)) as per definition of Game $(3, i)$. This means that prior to submitting the $i$-th Type 2 verification query, the adversary has gained no information about the $x_{\text {id }}$, id $\in[\mathrm{C}]$, and thus about the secret $x_{\text {id }}$, id $\in \mathrm{I}$. Hence, $\operatorname{Pr}[\operatorname{Bad}]=\frac{d}{p}$, which implies that for every $i \in\left[Q_{\text {Ver }}\right]$ it holds that $\left|\operatorname{Pr}\left[G_{3, i-1}(\mathcal{A})\right]-\operatorname{Pr}\left[G_{3, i}(\mathcal{A})\right]\right| \leq \frac{d}{p}$. By summing up for all the $Q_{\text {ver }}$ hybrids we get

$$
\left|\operatorname{Pr}\left[\mathrm{G}_{3}(\mathcal{A})\right]-\operatorname{Pr}\left[\mathrm{G}_{\left(3, Q_{\mathrm{Ver}}\right)}(\mathcal{A})\right]\right| \leq Q_{\mathrm{Ver}} \cdot \frac{d}{p} .
$$


Chapter 4. Extending Functionalities: Multi-Key Homomorphic Authenticators

By also considering equation (4.11) the above formula gives the bound stated in Lemma 34.

Combining the results of the lemmas we obtain that for every $\operatorname{PPT} \mathcal{A}$,

$$
\mathbf{A d v}_{\mathrm{MKHAut}, \mathcal{A}}^{\mathrm{HomUU}}(\lambda) \leq\left(Q_{\mathrm{Ver}}+1\right) \cdot Q_{\mathrm{id}} \cdot \mathbf{A d v}_{F, \mathcal{D}}^{P R F}(\lambda)+\frac{\left(Q_{\mathrm{Ver}}^{2}+2 \cdot Q_{\mathrm{Ver}}\right) \cdot d}{p}
$$

which proves Theorem 21 . 


\section{Extended Fair Exchange Over the Bitcoin Blockchain: Zero Knowledge Contingent Payments for Services}

As we already discussed in the introduction, in this chapter we present our solution to the problem of fair exchange over the Bitcoin Blockchain.

We already gave an intuitive description of how Zero Knowledge Contingent Payments works, and we also sketched which are the weaknesses in terms of design and functionalities, and how we manage to overcome them. In this Chapter we are giving a more detailed description of our results, along with all the technical details.

\subsection{An Overview of Our Contribution}

The advancements presented in this thesis address different aspects of the problem of fair exchange over the Bitcoin Blockchain and can be summarized as follows:

Attacks and fixes on ZKCP. We show that the ZKCP protocol when instantiated as it is now, is insecure, and develop several concrete attacks that allow a malicious buyer to learn information about the witness without paying the seller. We implement our attack by writing code for a malicious buyer that interacts with the unmodified implementation of the seller [36], and learns information about the Sudoku solution. We discuss how to avoid these attacks and various possible solutions.

Zero-Knowledge Contingent Service Payments. We introduce this new notion, and provide protocols for ZKCSP in both the public and private verifier setting. Again using our PoR example, the public verifier setting is when one wants to perform the service for the general public. The private verifier setting is when one wants to provide the service only for a specific individual. 
Implementation. We implemented and tested the ZKCP attack. We also implemented and tested our two new ZKCSP protocol, for the case of PoR, showing that they are feasible. Moreover we implemented a secure Pay-to-Sudoku ZKCP via our ZKCSP protocol. Our code is available here [41, 42].

Improved SHA256 circuit. In the process of our implementation of the ZKCSP protocols, we built a library for semi-automated boolean circuit generation. The SHA256 circuit that we produce has 22,272 AND gates, whereas the best publicly available circuit had 90,825 AND gates [109]. We released our SHA256 circuit together with our code as it may be of independent use for circuit-based MPC and FHE protocols that require SHA256 circuits.

\subsubsection{Related work}

In [16], Banasik et al. provide a ZKCP solution which avoids the use of NIZK by replacing the zero knowledge proof with an interactive protocol performed online. Moreover they avoid using hash-locked transactions since they claim that they are not standard and widely accepted in the Bitcoin network 1 .

The protocol presented in [16] is vulnerable to the so-called mauling problem, where an adversary which knows the hash identifier $T$ of a transaction is able to come up with a hash identifier $T^{\prime}$ that is semantically equivalent to $T$ (i.e. spends the same transaction, has the same value, and the same inputs and outputs). As the authors of [16] point out, there are many Bitcoin software clients that cannot handle transactions appearing in the ledger with an hash identifier which is different from the original one (namely, the one with which they were posted) [10]. This effectively makes the transaction unredeemable, causing problems when creating Bitcoin contracts [9, 10]. While the authors acknowledge the mauling problem, their scheme only addresses mauling due to malleability in ECDSA signatures, but does not address mauling due to changing the script.

An Ethereum-based contingent payment protocol is described by Tramer et al. in [110].

\subsection{Preliminaries}

In this section we present some concepts and basic definitions which will be frequently used in the rest of the chapter.

\subsubsection{Bitcoin and Ethereum}

Bitcoin is a decentralized digital currency proposed in 2008 [100. We present only the necessary background for this work here, but refer the reader to [35] and [101] for a detailed treatment.

\footnotetext{
${ }^{1}$ To the best of our knowledge, this is not really a serious issue.
} 
Bitcoins are typically associated with addresses, and an address is just a hash of a public key. To transfer Bitcoins from one address to another, one crafts a transaction which lists one or more input addresses from which the funds will be taken and one or more output addresses to which the funds will be sent.

In order for a transaction to be valid, the transaction must be signed with the private keys corresponding to the input addresses, the sum of the outputs must be less than or equal to the sum of the inputs, and the inputs must not have previously been spent [100, 24].

Signed transactions are broadcast to the Bitcoin peer-to-peer network. Miners check the validity of transactions and group them into blocks. Miners participate in a distributed consensus protocol that chains these blocks into an append-only global ledger called the Blockchain.

What we've described so far is a typical Bitcoin transaction, known as a Payto-PubkeyHash transaction. However, for each output, the transaction includes a script written in a stack-based programming language that specifies the conditions which must be met in order to spend this output in the future. For each input address, the transaction contains a reference to a previous transaction which listed this address as an output and specified the conditions required for it to be spent.

For a Pay-to-PubkeyHash transaction, the output script simply specifies an address and that in order to spend this output, one must sign with the associated private key. But Bitcoin scripts can be more complex as well. The Bitcoin scripting language has a limited set of op_codes or built-in functions that can be used to create scripts.

Using the The OP_ SHA256 op_code, the Bitcoin scripting language supports hash-locked transactions that specify a value $y$ and require that in order to spend this output, one must provide an $x$ such that $\operatorname{SHA256}(x)=y$.

A feature that was not initially included in the scripting language but introduced in 2012 is Pay-to-ScriptHash (P2SH) addresses. To redeem an output sent to a P2SH address, one must specify a script that hashes to this address, and then meet the conditions specified in the script[26].

Bitcoin scripts now also support other two op_codes: OP_CHECKLOCKTIMEVERIFY and OP_CHECKSEQUENCEVERIFY. The op_codes allow one to specify execution paths in the spending scripts that can only be validated after some relative or absolute time. For example, one can send money to Alice's address and specify that after 24 hours if Alice has not redeemed the output, then Bob can claim it by signing with his private key[26].

Although miners will accept the validity of all transactions that Bitcoin supports when included in blocks that others mine, most miners will only include a smaller subset of those transactions in the blocks that they construct. These are referred to "standard" transactions, and historically, this mean that it was quite difficult to get nonstandard transactions onto the Blockchain. In Bitcoin today, however, this is no longer an issue since almost all scripts are now considered standard when 
they are part of a P2SH transaction [8]. While Bitcoin's scripting language contains another useful op_code, it is not a Turing-complete language and is limited in practice. Ethereum is another cryptocurrency with a much more expressive scripting language that allows one to express arbitrary programs as conditions for spending money. As transactions can specify arbitrary scripts, there is no guarantee that they will ever halt. Each Ethereum transaction therefore contains gas, or money that is sent to the miner to run the transaction. Every computational step has a fixed gas cost, and the miner will only run the computation until it runs out of gas.

There is a global gas limit that specifies a maximum amount of gas that can be spent in a single block, and consequently in a single transaction. Although in theory Ethereum scripts can support arbitrary programs, the current gas limits are quite restrictive and do not allow for complex computations.

\subsubsection{Claw free function pairs}

Informally one can think of claw free function pairs as pairs of efficiently computable functions $H_{1}, H_{2}$ such that it is hard to find $x_{1}, x_{2}$ with $H_{1}\left(x_{1}\right)=H_{2}\left(x_{2}\right)$.

Definition 24. Let $C F G(\cdot)$ be an efficient algorithm that on input of a security parameter $1^{n}$ outputs two functions $H_{1, n}$ and $H_{2, n}$ with domain and image $\{0,1\}^{n}$. We say that $C F G(\cdot)$ is a claw free function generator, and $H_{1, n}, H_{2, n}$ are a clawfree pair if

- $H_{1, n}$ and $H_{2, n}$ can be efficiently computed

- for any efficient algorithm $\mathcal{A}$ we have that for $\left(H_{1, n}, H_{2, n}\right) \leftarrow C F G\left(1^{n}\right)$

$$
\begin{array}{r}
\operatorname{Pr}\left[\mathcal{A}\left(H_{1, n}, H_{2, n}\right)=\left(x_{1}, x_{2}\right)\right. \text { s.t. } \\
\left.H_{1, n}\left(x_{1}\right)=H_{2, n}\left(x_{2}\right)\right] \leq \operatorname{negl}(n)
\end{array}
$$

Computational Indistinguishability Recall that two distributions are said to be computationally indistinguishable if no efficient algorithm can distinguish if elements are sampled according to one or the other distribution.

Definition 25. Let $\mathcal{D}_{1, n}, \mathcal{D}_{2, n}$ be two (family of) distributions defined over $\{0,1\}^{n}$. We say that $\mathcal{D}_{1, n}, \mathcal{D}_{2, n}$ are computationally indistinguishable if for any efficient algorithm $\mathcal{A}$ we have that

$$
\begin{array}{r}
\mid \operatorname{Pr}\left[x \leftarrow \mathcal{D}_{1, n} ; \mathcal{A}(x)=1\right]-\operatorname{Pr}\left[x \leftarrow \mathcal{D}_{2, n} ;\right. \\
\mathcal{A}(x)=1] \mid \\
\leq \operatorname{negl}(n)
\end{array}
$$




\subsubsection{Fair Exchange}

In this section we recall the definition of fair exchange following previous work in [11, 90]. We have two parties Alice and Bob who want to exchange generic digital items. We know, due to a classic result of Cleve [53], that in the presence of malicious parties a fair exchange is impossible: one party will always have an advantage over the other. The traditional way to solve this problem is to rely on an Arbiter, a trusted third party (TTP), which is assumed to be honest and will help Alice and Bob exchange the items fairly. An optimistic fair exchange protocol involves the Arbiter only if one of the two parties does not behave honestly and complications arise. Two honest parties can exchange goods without involving the Arbiter.

The full definition in [11, 90] involves also two other parties (assumed to also be honest): a Tracker and a Bank. The former is used to make sure that the goods exchanged by the parties are the correct ones, while the latter takes care of eventual payments and money exchanges. The verification of the digital goods is executed by the Tracker in a trusted off-line phase where parties are provided with "verification keys" for the digital goods. For brevity's sake we are not going to describe this part and refer the reader to [11, 90]. Instead we just assume that Alice's and Bob's inputs include these verification keys, together with some public parameters.

Definition 26. A fair exchange protocol is a three-party communication protocol: Alice running algorithm A, Bob running an algorithm B, and the Arbiter running a trusted algorithm T. All parties run on input some public parameters PP, Alice runs on input $f_{A}, V_{A}$, Bob runs on input $f_{B}, V_{B}$, and the Arbiter runs on a input $s k_{T}$.

We denote with $[a, b] \leftarrow\left[A\left(f_{A}, V_{A}\right), B\left(f_{B}, V_{B}\right), T\left(s k_{T}\right)\right]$ the event that at the end of the execution of the protocol Alice outputs $a$ and Bob outputs $b$, where $a, b$ can be $\perp$ meaning that the parties reject the execution (e.g. their output is not valid according to their verification key - we assume that the files $f_{A}, f_{B} \neq \perp$ ).

Completeness: A fair exchange protocol is complete if the execution of the protocol by honest parties results in Alice getting Bob's files and viceversa:

$$
\operatorname{Pr}\left[\left[f_{B}, f_{A}\right] \leftarrow\left[A\left(f_{A}, V_{A}\right), B\left(f_{B}, V_{B}\right), T\left(s k_{T}\right)\right]\right]=1
$$

We say that a fair exchange is optimistic if the algorithm $T$ is not invoked by the correct algorithms $A$ and $B$.

Fairness: Intuitively, fairness states that, at the end of the protocol, either Alice and Bob get valid content (that is, content which passes the verification algorithm they were given by the Tracker), or neither Alice nor Bob get anything which passes the verification procedure. The above informal notion of fairness however does not capture the notion of partial information. It could be that a 
possibly malicious $\hat{B}$ learns something about a valid $f_{A}$ while $A$ outputs $\perp$. We strengthen the definition of fairness to capture the fact that if an honest party outputs $\perp$ then the other party learns no information. This is captured by a standard simulation definition. We say that a protocol is fair if for all efficient algorithms $\hat{B}$ there exists an efficient simulator $\operatorname{Sim}_{\hat{B}}$ with oracle access to $T$ such that the two distributions

$$
\left[\perp, \operatorname{Sim}_{\hat{B}}^{T}\left(f_{B}, V_{B}, V_{A}\right)\right]
$$

and

$$
[\perp, b] \leftarrow\left[A\left(f_{A}, V_{A}\right), \hat{B}\left(f_{B}, V_{B}\right), T\left(s k_{T}\right)\right]
$$

are computationally indistinguishable. A dual condition must hold for any possibly malicious efficient $\hat{A}$.

\subsubsection{Smart Contracts: Fair Exchange over Blockchains}

Assume that the exchange is a typical marketplace transaction, where $A$ is a seller, $f_{A}$ is a digital good, $B$ is a buyer, and $f_{B}$ is money. If the money is implemented via a Blockchain-based digital currency such as Bitcoin, then one can leverage the assumption that the Blockchain is a trusted "entity" and use it as the arbiter in a fair exchange protocol. Since the Blockchain is involved in the transaction anyway, to transfer the money from the buyer to the seller, we can dispense with the optimistic feature, and just use a protocol which always uses the arbiter.

These types of fair exchange over a Blockchain have been called smart contracts and can be abstracted to work in the following way. The buyer $B$ posts a transaction on the Blockchain that basically says

Transfer $f_{B}$ coins to the party who presents a string $f$ that satisfies the verification algorithm $V_{B}$

Then $A$ can post a transaction that says

Here is $f_{A}$ that satisfies $V_{B}$. Transfer those $f_{B}$ coins to my address.

This type of transactions can be implemented over Blockchains with sufficiently rich scripting languages: recall that a script is the program that needs to be executed in order to spend an output on the Blockchain. The scripting language in Ethereum [40, 115] is sufficiently rich, and one can in theory run any program as part of a transaction, which allows the execution of arbitrary contracts. In practice, the gas cost and global gas limit the complexity of Ethereum scripts.

In the simplified transactions above, everybody will learn the object $f$ being purchased by $B$. But this problem can be avoided by changing the verification procedure accordingly. $B$ could request that the object $f$ being purchased be 
encrypted under his public key, and published together with a non-interactive zero-knowledge proof that $f$ satisfies the verification algorithm $V_{B}$. Note that the latter is an NP statement so (at least in theory) it can be proven in zeroknowledge. One interesting issue (which we discuss in Section 5.3 is how to actually implement this NIZK proof, and in particular the selection of the common reference string that is needed by such proofs.

This type of smart contracts that allow parties to buy and sell knowledge in a trustless manner have been named Zero-Knowledge Contingent Payments $(Z K C P)$, and as we will show below ZKCP protocols have been proposed over Blockchain systems with more limited scripting language like Bitcoin [25].

\subsubsection{Zero-Knowledge Contingent Payments: Fair Exchange over Bitcoin}

The problem with the smart contract described above is that it is not possible to implement it directly in a Bitcoin transaction since the scripting language does not allow arbitrary verification procedures. Recall from Section 5.2.1 that a hash-locked transaction allows a party to redeem a transaction output if he/she produces the preimage (under SHA256) of a specific hashed value included in the original transaction.

Using hash-locked transactions the following construction was originally presented by Maxwell in 2011, and is now well known in the Bitcoin community [25, 94]: Alice (seller) and Bob (buyer) engage in an offline phase, where Alice encrypts the string $f_{A}$ with a key $k$ (using any symmetric encryption scheme $E$, i.e. AES) and publishes $\hat{f}=E_{k}\left(f_{A}\right)$ and $s=\operatorname{SHA256}(k)$ together with a ZK proof that $E_{\mathrm{SHA256}}^{-1}(s)(\hat{f})$ satisfies the verification procedure $V_{B}$. Again this is an NP statement and therefore can be proven in ZK. Since this interaction between Alice and Bob will not be posted on the Blockchain, the proof could be performed interactively or non-interactively.

If the proof is correct, Bob then broadcasts the following transaction to be included in the Blockchain:

Transfer $f_{B}$ Bitcoins to the party who presents a SHA256 preimage of $s$ and signs the transaction with $p k_{\text {Alice }}$. If this output is still unspent after $n$ blocks, then the Bitcoins can be claimed by $p k_{B o b}$.

At this point Alice can claim the coins by signing the transaction that publishes $k$, which in turn will allow Bob to recover the digital good $f_{A}$.

Note that the transaction that Bob posts requires that the seller provides both the preimage $k$ as well as a signature. The reason that we also require a signature is to prevent a front-running attack in which Alice broadcasts $k$ to the network to claim the funds, but before Alice's transaction is included in a block, some other party (perhaps the miner) sees $k$ and uses it to claim the funds for 
themselves. To prevent this attack, the transaction requires Alice's signature as well, which nobody else can produce.

Also notice the second condition in the transaction that specifies that after a certain amount of time elapses, Bob can himself claim the output of this transaction. This is a refund clause that allows Bob to reclaim his output in case Alice decides not to post $k$. Without this clause, in the event that Alice decides not to complete the protocol and publish $k$, Bob's funds would be locked up and he would neither have his money nor the string $f_{A}$.

\subsubsection{Example: Pay for Sudoku Solutions}

When Maxwell first proposed ZKCP in 2011 it was only theoretical as there was no known efficient general purpose zero-knowledge protocol that could be used. But advances since then in zero knowledge protocols [68, 55, 20] have made this protocol feasible and indeed there is currently a publicly available implementation of ZKCP for purchasing Sudoku solutions. Using the template above, the string $f_{A}$ is the solution of an $n \times n$ input Sudoku table (which also specifies the verification algorithm $V_{B}$ ). The main challenge of course is the implementation of the ZK proof that the decryption of $\hat{f}$ under the preimage of $s$ is indeed a valid Sudoku solution for the input Sudoku table. They implemented this non-interactively, using the ZK Succint Non-Interactive Arguments of Knowledge (ZK-SNARK) based on Quadratic Arithmetic Programs [68, 102], using the libsnark library [18, 21].

As with all NIZK proofs, QSP-based ZK-SNARGs require a common reference string (CRS) for the production and the verification of the proof $\mathrm{f}^{2}$. Such CRS should be selected by a trusted party in advance, which is obviously non-ideal for ZKCP. The entire premise of ZKCP is to perform fair-exchange over the Blockchain in a trustless manner, and introducing a trusted third party would largely defeat the purpose.

To get around this, it was noticed that unlike proofs which are produced to be verified by the public, the ZK-proof in ZKCP only need to convince a single person - the buyer. In ZKCP it was therefore proposed that the buyer (i.e. the verifier) generate the CRS, to ensure that the seller could not cheat.

However, having the buyer generate the CRS is problematic as it only protects against a soundness adversary but not a zero-knowledge adversary. With regards to the proof's soundness property, the seller is the adversary as the seller would benefit from producing an incorrect proof. However, with regards to the proof's zero-knowledge property, the buyer is the adversary as the buyer would benefit from learning some information about $f_{A}$ without paying for it. If one generates the CRS maliciously, and (as we show below) the CRS is not checked for "correctness", they can break both soundness and zero-knowledge.

\footnotetext{
${ }^{2}$ In the SNARKs literature, the CRS is sometimes referred to as the proving key and the verifying key.
} 
Because the ZKCP protocol does not check the correctness of the CRS it only ensures that the seller can not cheat, but it allows the buyer to cheat and extract information about the witness $f_{A}$ without paying for it. In the next section we use this fact to show a concrete attack on the ZKCP protocol that leaks information about the value of a Sudoku cell before the buyer pays for the solution.

\subsection{Attacks on ZKCP with untrusted CRS}

In this section we show how allowing the Verifier to choose the CRS in the QAPbased SNARK leads to a loss of the Zero-Knowledge property. While it is a well known fact in the cryptographic literature that a trusted CRS is needed for zero-knowledge, the point of this section is to demonstrate this insecurity by developing concrete attacks that allow one to learn information in the "Pay-toSudoku" implementation, where the Verifier does indeed set the CRS. Through our attack, the Verifier is able to verify if a particular guess for a Sudoku cell is correct or not. This obviously break the fairness of the protocol (as defined in Section 5.2.3 since the buyer learns partial information about the seller's input.

First we recall how Quadratic Arithmetic Span programs work, since they are the proof backbone of the libsnark library used in the implementation. Then we show our attacks, and describe our implementation of the malicious verifier.

\subsubsection{ZK-SNARKs from Quadratic Arithmetic Programs}

We recall here the notion of Quadratic Arithmetic Programs (QAPs) [68, 102], using the notation of Ben-Sasson et al. [21].

Definition 27 ([68]). A $Q A P \mathcal{Q}$ over a field $\mathbb{F}$ is defined by three sets of polynomials $A:=\left\{A_{i}(x)\right\}_{i=0}^{m}, B:=\left\{B_{i}(x)\right\}_{i=0}^{m}, C:=\left\{C_{i}(x)\right\}_{i=0}^{m}$ and a target polynomial $Z(x)$. If we take a function $f: \mathbb{F}^{n} \rightarrow \mathbb{F}^{n^{\prime}}$, then we say that $\mathcal{Q}$ computes $f$ if, given a valid assignment $\left(c_{1}, \ldots, c_{n+n^{\prime}}\right)$ of inputs and outputs of $f$, there exist coefficients $\left(c_{n+n^{\prime}+1}, \ldots, c_{m}\right)$ such that $Z(x)$ divides the following polynomial

$$
\begin{aligned}
p(x):=\left(A_{0}(x)+\right. & \left.\sum_{k=1}^{m} c_{k} \cdot A_{k}(x)\right) \cdot\left(B_{0}(x)+\sum_{k=1}^{m} c_{k} \cdot B_{k}(x)\right)+ \\
& -\left(C_{0}(x)+\sum_{k=1}^{m} c_{k} \cdot C_{k}(x)\right)
\end{aligned}
$$

In other words there must exists a polynomial $H(x)$ such that $p(x)=H(x)$. $Z(x)$. We refer to $m$ and the degree of $Z(x)$ as the size and the degree of $\mathcal{Q}$ respectively.

To build a QAP for a function $f$, we use an arithmetic circuit $\mathcal{C}$ representing $f$; we then pick a distinct root $r_{g}$ for any of its multiplicative gates. Then, we 
Chapter 5. Extended Fair Exchange Over the Bitcoin Blockchain: Zero

Knowledge Contingent Payments for Services

build the target polynomial as $Z(z):=\prod_{g}\left(z-r_{g}\right)$, and we label each input of the circuit and each output of a multiplicative gate with an index $i \in[m]$ (grouping together all the additive gates). We define the polynomials $A, B, C$ in a way that they respectively encode the left, right and output wire of each gate: for example, $B_{i}\left(r_{g}\right)=1$ if the $i$-th wire of the circuit is a right input wire of the gate $g$, and $B_{i}\left(r_{g}\right)=0$ (and similarly with $A$ and $C$ with left input and output wires respectively). So, for any gate $g$ and its root $r_{g}$, the condition above can be seen as:

$$
\begin{gathered}
\left(\sum_{k=1}^{m} c_{k} \cdot A_{k}\left(r_{g}\right)\right) \cdot\left(\sum_{k=1}^{m} c_{k} \cdot B_{k}\left(r_{g}\right)\right)= \\
=\left(\sum_{k \in I_{L}} c_{k} \cdot A_{k}\left(r_{g}\right)\right) \cdot\left(\sum_{k \in I_{R}} c_{k} \cdot B_{k}\left(r_{g}\right)\right)=c_{g} C_{k}\left(r_{g}\right)=c_{g}
\end{gathered}
$$

which basically says that the output of a multiplication gate is the multiplication between the values on the left and the right inputs wire of the gate itself. Following the notation of [21], it is now possible to use QAPs to build zk-SNARKs, as in [68, 102]:

Public Parameters: pp $:=\left(r, e, \mathcal{P}_{1}, \mathcal{P}_{2}, \mathbb{G}_{1}, \mathbb{G}_{2}, \mathbb{G}_{T}\right)$ where $\mathbb{G}_{1}:=$ $\left\langle\mathcal{P}_{1}\right\rangle, \mathbb{G}_{2}:=\left\langle\mathcal{P}_{2}\right\rangle, \mathbb{G}_{T}$ are groups of prime order $r$ and $e: \mathbb{G}_{1} \times \mathbb{G}_{2} \rightarrow \mathbb{G}_{T}$ is a pairing.

Key Generation The key generation procedure is composed by several steps, it takes in input a circuit $\mathcal{C}: \mathbb{F}_{r}^{n} \times \mathbb{F}_{r}^{h} \rightarrow \mathbb{F}_{r}^{\ell}$ and outputs a proving key $p k$ and a verification key $v k$.

1. Compute $(A, B, C, Z)$ with respect to the circuit $\mathcal{C}$ and extend $A:=$ $\left\{A_{i}(x)\right\}_{i=0}^{m}, B:=\left\{B_{i}(x)\right\}_{i=0}^{m}, C:=\left\{C_{i}(x)\right\}_{i=0}^{m}$ via $A_{m+1}=B_{m+2}=$ $C_{m+3}=Z, A_{m+2}=A_{m+3}=B_{m+1}=B_{m+3}=C_{m+1}=C_{m+2}=0$.

2. Sample $\tau, \varphi_{A}, \varphi_{B}, \alpha_{A}, \alpha_{B}, \alpha_{C}, \beta, \gamma \stackrel{\$}{\leftarrow} \mathbb{F}_{r}$

3. For $i=0, \ldots, m+3$, let

$$
\begin{aligned}
& p k_{A, i}:=A_{i}(\tau) \varphi_{A} \mathcal{P}_{1}, \quad p k_{A, i}^{\prime}:=A_{i}(\tau) \alpha_{A} \varphi_{A} \mathcal{P}_{1} \\
& p k_{B, i}:=B_{i}(\tau) \varphi_{B} \mathcal{P}_{2}, \quad p k_{B, i}^{\prime}:=B_{i}(\tau) \alpha_{B} \varphi_{B} \mathcal{P}_{1} \\
& p k_{C, i}:=C_{i}(\tau) \varphi_{C} \mathcal{P}_{1}, \quad p k_{C, i}^{\prime}:=C_{i}(\tau) \alpha_{C} \varphi_{A} \varphi_{B} \mathcal{P}_{1} \\
& p k_{K, i}:=\beta\left(A_{i}(\tau) \varphi_{A}+B_{i}(\tau) \varphi_{B}+C_{i}(\tau) \alpha_{C} \varphi_{A} \varphi_{B}\right) \mathcal{P}_{1} \\
& \text { and for } i=0, \ldots, d \text { let } p k_{H, i}:=\tau^{i} \mathcal{P}_{1} . \text { Set } \\
& p k:=\left(\mathcal{C}, p k_{A}, p k_{A}^{\prime}, p k_{B}, p k_{B}^{\prime}, p k_{C}, p k_{C}^{\prime}, p k_{K}, p k_{H}\right) .
\end{aligned}
$$


4. Let

$$
\begin{aligned}
& v k_{A}:=\alpha_{A} \mathcal{P}_{2}, v k_{B}:=\alpha_{B} \mathcal{P}_{1}, v k_{C}:=\alpha_{C} \mathcal{P}_{2} \\
& v k_{\gamma}:=\gamma \mathcal{P}_{2}, v k_{\gamma \beta}^{1}:=\gamma \beta \mathcal{P}_{1}, v k_{\gamma \beta}^{2}:=\gamma \beta \mathcal{P}_{2} \\
& v k_{Z}:=Z(\tau) \varphi_{A} \varphi_{B} \mathcal{P}_{2} \\
& \left\{v k_{I C, i}\right\}_{i=0}^{n}:=\left\{A_{i}(\tau) \varphi_{A} \mathcal{P}_{1}\right\}_{i=0}^{n} .
\end{aligned}
$$

Set

$$
v k:=\left(v k_{A}, v k_{B}, v k_{C}, v k_{\gamma}, v k_{\gamma \beta}^{1}, v k_{\gamma \beta}^{2}, v k_{Z}, v k_{I C}\right) .
$$

5. Output $(p k, v k)$

Prover: On input a proving key $p k$, an input $x \in \mathbb{F}_{r}^{n}$, a witness $a \in \mathbb{F}_{r}^{h}$, it outputs a proof $\pi$ which is computed as follows:

1. Compute $(A, B, C, Z)$ with respect to the circuit $\mathcal{C}$.

2. Compute the QAP witness $s \in \mathbb{F}^{m}$ with respect to $\mathcal{C}, x, a$.

3. Sample $\delta_{1}, \delta_{2}, \delta_{3} \stackrel{\$}{\leftarrow} \mathbb{F}_{r}$.

4. Compute the polynomial

$$
H(z):=\frac{A(z) B(z)-C(z)}{Z(z)}
$$

where

$$
\begin{aligned}
& A(z):=A_{0}(z)+\sum_{i=1}^{m} s_{i} A_{i}(z)+\delta_{1} Z(z), \\
& B(z):=B_{0}(z)+\sum_{i=1}^{m} s_{i} B_{i}(z)+\delta_{2} Z(z), \\
& C(z):=C_{0}(z)+\sum_{i=1}^{m} s_{i} C_{i}(z)+\delta_{3} Z(z) .
\end{aligned}
$$

and represent $H(z)$ as $\left(h_{0}, \ldots, h_{d}\right) \in \mathbb{F}_{r}^{d+1}$

5. Set

$$
\begin{aligned}
\tilde{p k}_{A} & :=\left(0^{n}, p k_{A, n+1}, \ldots, p k_{A, m+3}\right) \\
\tilde{p k}_{A}^{\prime} & :=\left(0^{n}, p k_{A, n+1}^{\prime}, \ldots, p k_{A, m+3}\right) .
\end{aligned}
$$

6. Let $c:=\left(1, s, \delta_{1}, \delta_{2}, \delta_{3}\right) \in \mathbb{F}_{r}^{4+m}$, compute 


$$
\begin{aligned}
\pi_{A}:=\left\langle c, \tilde{p k_{A}}\right\rangle, & \pi_{A}^{\prime}:=\left\langle c, \tilde{p k}_{A}^{\prime}\right\rangle, \\
\pi_{B}:=\left\langle c, p k_{B}\right\rangle, & \pi_{B}^{\prime}:=\left\langle c, p k_{B}^{\prime}\right\rangle, \\
\pi_{C}:=\left\langle c, p k_{C}\right\rangle, & \pi_{C}^{\prime}:=\left\langle c, p k_{C}^{\prime}\right\rangle, \\
\pi_{K}:=\left\langle c, p k_{K}\right\rangle, & \pi_{H}:=\left\langle h, p k_{K}\right\rangle .
\end{aligned}
$$

7. Output $\pi:=\left(\pi_{A}, \pi_{A}^{\prime}, \pi_{B}, \pi_{B}^{\prime}, \pi_{C}, \pi_{C}^{\prime}, \pi_{K}, \pi_{H}\right)$.

Verifier: On input a verification key $v k$, an input $x \in \mathbb{F}_{r}^{n}$ and a proof $\pi$, the verifier proceeds as follows:

1. Compute $v k_{x}:=v k_{I C, 0}+\sum_{i=1}^{m} x_{i} v k_{I C, i} \in \mathbb{G}_{1}$.

2. Verify validity of knowledge commitments for $A, B, C$ by checking:

$$
\begin{aligned}
& e\left(\pi_{A}, v k_{A}\right)=e\left(\pi_{A}^{\prime}, \mathcal{P}_{2}\right), \quad e\left(\mathbf{v k}_{B}, \pi_{B}\right)=e\left(\pi_{B}^{\prime}, \mathcal{P}_{2}\right), \quad e\left(\pi_{C}, v k_{C}\right)= \\
& e\left(\pi_{C}^{\prime}, \mathcal{P}_{2}\right) \text {. }
\end{aligned}
$$

3. Verify that the same coefficients were used by checking:

$$
e\left(\pi_{K}, v k_{\gamma}\right)=e\left(v k_{x}+\pi_{A}+\pi_{C}, v k_{\gamma \beta}^{2}\right) \cdot e\left(v k_{\gamma \beta}^{1}, \pi_{B}\right) .
$$

4. Check QAP divisibility

$$
e\left(v k_{x}+\pi_{A}, \pi_{B}\right)=e\left(\pi_{H}, v k_{Z}\right) \cdot e\left(\pi_{C}, \mathcal{P}_{2}\right)
$$

5. Output 1 (accept) if and only if all the above checks are satisfied.

\subsubsection{Learning Information by modifying the CRS}

If a possibly malicious Verifier is allowed to set the CRS (as in the "Pay to $\mathrm{Su}$ doku" (PtS) code [36]), then there are a variety of attacks that can be attempted to learn information about the Sudoku solution during the offline phase of the ZKCP (and therefore before the payment phase is completed).

Changing the Circuit This is the easiest attack to consider. Recall that the CRS of a QAP-based SNARK consists of an encoding of a QAP encoding of the function $f$ that verifies the NP witness held by the prover. A malicious verifier could just replace the CRS with the QAP encoding of a modified function $\tilde{f}$ whose output directly leaks the needed information. In other words, the sets of polynomials $A, B, C$ and $C$, and the polynomial $Z$ would be modified to $\tilde{A}, \tilde{B}, \tilde{C}, \tilde{Z}$. Nevertheless this trivial attack does not work in a libsnark implementation of 
QAP-based SNARKs. The reason is that the QAP-encoding of a function $f$ is a deterministic process, and in libsnark both prover and verifier compute the polynomials $A, B, C, Z$ on their own directly from a description of the function $f$, and this leads to a straightforward detection of any change.

Learning one wire is sufficient We now point out that in the PtS implementation, for every Sudoku cell, there are $n$ wires $w_{1}, \ldots, w_{n}$ in the circuit $\mathcal{C}$ used in the SNARK, such that $w_{j}=1$ if the cell is set to $j$ in the solution, while all the other wires related to that cell are set to 0 . Therefore learning the value of the wire $w_{j}$ will allow us to learn if that particular cell is set to $j$ or not. Recall from the previous section that the value of the wires of $\mathcal{C}$ are the coefficients $c_{i}$ used to compute the linear combinations so it is sufficient to learn $c_{j}$. Note also that $c_{j}$ can only assume a binary value. We now focus on attacks that allow us to compute a single coefficient $c_{j}$.

Choosing $\tau$ as one of the roots of $Z$ In the correct CRS generation, $\tau$ is chosen at random in the field $\mathbb{F}_{r}$. It turns out that if one selects $\tau$ as one of the roots of $Z(x)$, then $\tau$ is also the root of all the polynomials $A, B, C$ except for one of them, say $B_{j}(x)$, for which $B_{j}(\tau) \neq 0$. In this case the component $\pi_{B}$ of the proof produced by the prover reveals the value $\gamma_{j}=c_{j} \phi_{B} \mathcal{P}_{2}$ which allows to recover $c_{j}$ since it can only assume a binary value. This attack is not detected in libsnark on the prover side (it would be easily detected by checking the public key $p k$ and see if it contains the identity in either $\mathbb{G}_{1}$ or $\mathbb{G}_{2}$, but this check is not performed in libsnark). However the attack does not work in the "Pay-toSudoku" ZKCP for a very interesting reason. The prover code actually produces the "wire value leaking" proof $\pi_{B}$ without an error, but then before sending it out to the Verifier, the PtS code has the Prover run a verification of its own proof $\pi$. This verification fails because the polynomial $H(x)$ is computed by dividing via the polynomial $Z(x)$ and so when evaluated at $\tau$ the QAP divisibility check fail. Moreover, because of an optimization step of the verification procedure that does not expect to compute a pairing operation where the input in $\mathbb{G}_{2}$ is the identity, the proof fails even before getting to the QAP divisibility check (this will happen in the verification equation since $Z(\tau)=0$ implies that $v k_{Z}=0 \mathcal{P}_{2}=0 \in \mathbb{G}_{2}$ and this value is placed in the $\mathbb{G}_{2}$ pairing input of one of the verification equations).

Setting all the $p k$ equal to the identity, except for one wire This is the attack that works. The attack is described in detail below, but here we give an informal explanation. Here $\tau$ is selected at random, but it is not used to evaluate the polynomials. Similar to the attack above, the malicious verifier will set all the $p k_{A}, p k_{A}^{\prime}, p k_{C}, p k_{C}^{\prime} \in \mathbb{G}_{1}$ equal to 0 instead of setting them as the evaluation "in the exponent" of the polynomials $A, C$ evaluated at $\tau$. Similarly $p k_{B, i}=0 \in \mathbb{G}_{2}$ and $p k_{B, i}^{\prime}=0 \in \mathbb{G}_{1}$ for all $i \neq j$ and $p k_{B, j}=\varphi_{B} \mathcal{P}_{2}, p k_{B, j}^{\prime}=\alpha_{B} \varphi_{B} \mathcal{P}_{1}$ for known $\alpha_{B}, \varphi_{B}$. By setting the $p k, p k^{\prime}$ values this way, the proof $\pi$ will reveal the value $\gamma_{j}$ as above, and therefore the value $c_{j}$. Since the prover checks its own proof before releasing it, we need to make sure that the proof verifies. We do that by 
setting $p k_{H, i}=0 \in \mathbb{G}_{1}$ which will force the value $\pi_{H}$ produced by the prover to be $\pi_{H}=0 \in \mathbb{G}_{1}$. Moreover since all the identities are now only in the group $\mathbb{G}_{1}$, the error caused by the optimization in the libsnark implementation will not appear and indeed the proof is produced by the Prover (seller) and sent out to the Verifier (buyer), who will recover the value $c_{j}$.

More in details:

Public Parameters: Both the buyer and the prover get the public parameters and pp $:=\left(r, e, \mathcal{P}_{1}, \mathcal{P}_{2}, \mathbb{G}_{1}, \mathbb{G}_{2}, \mathbb{G}_{T}, \mathcal{C}\right)$ which include the description of the circuit $\mathcal{C}$.

Key Generation: The buyer takes the circuit $\mathcal{C}: \mathbb{F}_{r}^{n} \times \mathbb{F}_{r}^{h} \rightarrow \mathbb{F}_{r}^{\ell}$ and outputs a proving key $p k$ and a verification key $v k$ as follows:

1. Honestly computes $(A, B, C, Z)$ with respect to the circuit $\mathcal{C}$, where $A:=\left\{A_{i}(x)\right\}_{i=0}^{m}, B:=\left\{B_{i}(x)\right\}_{i=0}^{m}, C:=\left\{C_{i}(x)\right\}_{i=0}^{m}$.

Now he extends $A, B, C$ via

$A_{m+1}=A_{m+2}=A_{m+3}=0$,

$B_{m+1}=B_{m+2}=B_{m+3}=0$,

$C_{m+1}=C_{m+2}=C_{m+3}=0$.

2. Sample $\tau, \varphi_{A}, \varphi_{B}, \alpha_{A}, \alpha_{B}, \alpha_{C}, \beta, \gamma \stackrel{\$}{\leftarrow} \mathbb{F}_{r}$.

3. For $i=0, \ldots, m+3$, let

$p k_{A, i}:=0 \in \mathbb{G}_{1}, \quad p k_{A, i}^{\prime}:=0 \in \mathbb{G}_{1}$,

$p k_{B, i}:=0 \in \mathbb{G}_{2}$ for all $i \neq j$ and $p k_{B, j}:=\varphi_{B} \mathcal{P}_{2}$,

$p k_{B, i}^{\prime}:=0 \in \mathbb{G}_{1}$ for all $i \neq j$ and

$p k_{B, j}^{\prime}=\alpha_{B} \varphi_{B} \mathcal{P}_{1}$,

$p k_{C, i}:=0 \in \mathbb{G}_{1}, \quad p k_{C, i}^{\prime}:=0 \in \mathbb{G}_{1}$,

$p k_{K, i}:=0 \in \mathbb{G}_{1}$ for all $i \neq j, p k_{K, j}:=\beta \varphi_{B} \mathcal{P}_{1}$.

For $i=0, \ldots, d$ let $p k_{H, i}:=0 \in \mathbb{G}_{1}$, and set

$p k:=\left(p k_{A}, p k_{A}^{\prime}, p k_{B}, p k_{B}^{\prime}, p k_{C}, p k_{C}^{\prime}, p k_{K}, p k_{H}\right)$.

4. Let $v k_{A}:=\alpha_{A} \mathcal{P}_{2}, v k_{B}:=\alpha_{B} \mathcal{P}_{1}, v k_{C}:=\alpha_{C} \mathcal{P}_{2}$

$v k_{\gamma}:=\gamma \mathcal{P}_{2}, \quad v k_{\gamma \beta}^{1}:=\gamma \beta \mathcal{P}_{1}, \quad v k_{\gamma \beta}^{2}:=\gamma \beta \mathcal{P}_{2}$

$v k_{Z}:=Z(\tau) \varphi_{A} \varphi_{B} \mathcal{P}_{2}, \quad\left\{v k_{I C, i}\right\}_{i=0}^{n}:=\left\{0 \in \mathbb{G}_{1}\right\}_{i=0}^{n}$ and set

$v k:=\left(v k_{A}, v k_{B}, v k_{C}, v k_{\gamma}, v k_{\gamma \beta}^{1}, v k_{\gamma \beta}^{2}, v k_{Z}, v k_{I C}\right)$.

5. Output $(p k, v k)$

It is not hard to see that all the verification equations are satisfied, and that 
the proof leaks the value $c_{j}$. If used against the PtS code for contingent payments for Sudoku solutions, this attack allows to find out the value for a Sudoku cell with probability $1 / 9$. We provide an implementation for the attack above; see Section 5.5.1 for more details.

\subsubsection{Countermeasures}

In this section we show some possible countermeasures to our attack above.

Checking the CRS. As already discussed in the original paper on QSP/QAP 68] the prover can check that the CRS is "correctly formed" and in this case the protocol is witness indistinguishable (WI) [61]. In the QAP-based SNARK described in the previous section, it is sufficient that the prover/seller checks that

- The polynomials $A, B, C, Z$ are well formed with respect to the circuit $C$.

- The elements $p k_{A_{m+1}}, p k_{A_{m+1}}^{\prime}, p k_{B_{m+2}}^{\prime}, p k_{C_{m+3}}, p k_{C_{m+3}}^{\prime}$ are not equal to $0 \in \mathbb{G}_{1}$ and the element $p k_{B_{m+2}}$ is not equal to $0 \in \mathbb{G}_{2}$

- All the elements $p k_{H, i}$ are not $0 \in \mathbb{G}_{1}$.

- The element $v k_{Z}$ is such that $v k_{Z} \neq 0 \in \mathbb{G}_{2}$.

since this will guarantee that the proof is a uniformly distributed random value no matter what witness is used (see [68] ). This could be a good option for some applications of ZKCP, but unfortunately not for the PtS application since a Sudoku puzzle typically has only one solution and witness indistinguishability guarantees only that proofs "look the same" no matter what witness is used in the case that there are two or more such witnesses. It does not guarantee that no knowledge is leaked about a unique witness.

Subversion Resistant ZK. In a recent paper Bellare et al. introduce the notion of Subversion Zero Knowledge [17], i.e. the ability to prove ZK even when the CRS is maliciously selected by the verifier. Note that given some well known impossibility results [74, 73], the notion of ZK obtained in this case is somewhat weak (ZK does not hold with respect to arbitrary auxiliary inputs the verifier might have). One could then run a ZKCP with a subversion resistant ZK protocol.

The proposed solution in [17] is not a SNARK (the proof is not succinct), but it is not hard to see that their techniques extend to the original QSP/QAP protocol in [68]. Indeed subversion-ZK can be obtained as long as the above "WI checks" are performed and the value $\tau$ can be extracted by the simulator from the Verifier when it produces the CRS. Following the approach in [17] one could use a "knowledge of exponent" type of assumption to extract $\tau$ after checking that each $p k_{H, i}$ is correct, i.e $p k_{H, i}=\tau^{i} \mathcal{P}_{1}$. In the original QSP/QAP protocol in [68], where $\mathbb{G}_{1}=\mathbb{G}_{2}$, this can be checked using the bilinear map by checking that 
$e\left(\mathcal{P}_{1}, p k_{H, i}\right)=e\left(p k_{H, 1}, p k_{H, i-1}\right)$ for all $i$. The above intuition is actually formalized in [65] (a different subversion-ZK SNARK is presented in [1]).

Note that this check requires the computation of $\Theta(m)$ bilinear maps, a much more expensive task than the simple checks required for WI. Moreover it is not clear if those techniques extend to Pinocchio [102], the optimized version of the QSP/QAP protocol used by Libsnark [18, since in that case $\mathbb{G}_{1} \neq \mathbb{G}_{2}$ and the above check cannot be performed. Our experimental results suggests that running the subversion-resistant checks of [65] for the pay-to-sudoku example would take more than an hour on our benchmark machine (see Section 5.5.2 for full details).

So to summarize, one could obtain (a weak non-aux input notion of) zeroknowledge by using subversion resistant ZK, but it would require major changes in the current implementation of ZKCP protocols, and increase the computation required of the Prover.

Distributed Generation of the CRS. Another possible solution is to have buyer and seller run a two-party secure computation protocol to compute the CRS together. Note that due to the algebraic structure of the CRS, this could be done via a much more efficient ad-hoc protocol, rather than say a generic solution such as Yao's protocol. A similar approach was followed by the designers of Zcash [37, 19] to remove a trusted generation of the CRS in their the QAP-based SNARK: 3 .

Using Contingent Payments for Services. At the end the best solution in our opinion is to use the protocol for ZK Contingent Service Payments that we describe in the next Section.

\subsection{Contingent Service Payments}

In this section we discuss Contingent Payments for services such as auditing. Consider for example the case where Alice (the seller) is a data storage company, and Bob (the buyer) is a customer. Bob will store his files with Alice, and will pay her for this service. Assume that the contract between Bob and Alice is that periodically Alice will prove to Bob that his files are all correctly stored, and upon that proof Bob will pay the contracted rent.

There are several cryptographic protocols that allow a data storage provider to efficiently prove the integrity of the stored files to a customer. These are known under the name of Proofs of Retrievability (PoR) [86] and they all work by requiring that the prover shows the possession of a certain number of blocks

\footnotetext{
${ }^{3}$ We also point out that the CRS in their case is an "extended" version of the Pinocchio CRS, where both $\tau^{i} \mathcal{P}_{1}$ and $\tau^{i} \mathcal{P}_{2}$ appear in the CRS. This allows anybody to verify the correctness of the CRS via bilinear maps. Moreover, even if this CRS was computed by a single malicious party, rather than distributively, subversion ZK is guaranteed since the value $\tau$ can be extracted via a "knowledge of exponent" type of assumption.
} 
previously authenticated by the client 4 .

This can be achieved easily using a smart contract over a blockchain with a sufficiently rich scripting language. The client simply posts a transaction that pays whoever shows possession of such authenticated blocks. When the server posts those blocks, it will receive payment and the client will be assured all its files are still correctly stored.

But it does not work using the generic blueprint for ZKCP over Bitcoin described earlier. Indeed that blueprint requires the Server to prove possession of such blocks during the offline phase, but at this point the client has the desired knowledge (the server knows those blocks, therefore it must know the entire file) without having paid for it, and indeed the client does not have to post the payment transaction on the Bitcoin blockchain. The reason is that the ZKCP blueprint is designed for the sale of digital goods, but not digital services. In the ZKCP protocol described earlier the prover proves possession of a certain string, without revealing it, and the payment is contingent on the disclosure of the string. But in this case it's the proof of possession itself that is the valuable "service" desired by the buyer.

In the following we show how to design a ZKCP for digital services such as auditing and the other applications discussed in the Introduction.

\subsubsection{Defining ZKCP for Services (ZKCSP)}

We are looking for a protocol where a server $A$ proves to a client $B$ that he the server) knows $s$ such $f(s)=1$ for an efficiently computable verification function $f:\{0,1\}^{*} \rightarrow\{0,1\}$ and needs to be paid for this information. Informally the properties that we would like to have are

- If a possibly malicious $\hat{A}$ is paid then $\hat{A}$ must "known" a value $s$ such that $f(s)=1$;

- If a possibly malicious $\hat{B}$ does not pay then $\hat{B}$ has learned no information

- additionally, a possibly malicious $\hat{B}$ who pays, learns only that $A$ knows $s$ such that $f(s)=1$ and nothing else.

The latter condition can be relaxed in some settings, but by enforcing it we really limit the knowledge disclosure from $A$ to $B$ to a minimum.

We use a trusted party $T$ which models a blockchain. $T$ maintains a ledger of all the "coin balances" of each party. Moreover $T$ accepts messages from $A$ and $B$ of only two types, and will execute the instructions honestly:

4 Trivially the client can ask the prover to send back all the data originally stored and authenticated by the client, but this is not efficient. PoR protocols allow the server to prove that all the data is there by showing only a small number of blocks authenticated by the client (see appendix). 
Chapter 5. Extended Fair Exchange Over the Bitcoin Blockchain: Zero

Knowledge Contingent Payments for Services

Contingent Payments from $B$ which are of the form

Transfer $m$ of my coins to a party who publishes $x$ such that when you run this program $P$ on $x$ you get $P(x)=1$

In this case $T$ checks that $B$ has more than $m$ coins and if so accepts the message and publishes it on the blockchain, otherwise it rejects it.

Redemption Payments from $A$ which are of the form

Transfer $m$ coins to my account since I am publishing $x$ such that when you run $P$ on $x$ you get $P(x)=1$

In this case $T$ checks that there is a previously accepted Contingent Payment message that refers to this program $P$, and that $P(x)=1$. If so it will posts the message to the blockchain and will deduct $m$ coins from the balance of the party $B$ who posted the message, and adds has those $m$ coins to the balance of $A$.

A Zero-Knowledge Contingent Service Payment (ZKCSP) protocol is a three party protocol defined by the interactive machines $A, B, T$ where $A$ runs on a private input $s$, and all parties run on public input a function $f$. We define the view of $B, \operatorname{View}_{\hat{B}}(s, f)$ as his coin tosses together with all the messages exchanged during the protocol:

$$
\begin{aligned}
\text { View }_{\hat{B}}(s, f):= & \\
& {\left[\text { Coins }_{\hat{B}}|| \text { Messages }[A(s, f), \hat{B}(f), T(f)] \|\right.} \\
& \operatorname{Out}(A(s, f), \hat{B}(f), T(f)]
\end{aligned}
$$

We say that $(A, B, T)$ is a secure ZKCSP protocol if the following conditions are satisfied (all parties run on a security parameter $1^{n}$ )

Extraction For any possibly malicious efficient $\hat{A}$, if at the end of the protocol $\hat{A}$ 's balance increases with non-negligible probability, then there exists an efficient extractor $\operatorname{Ext}_{\hat{A}}$, which outputs a string $\hat{s}$ such that $f(\hat{s})=1$;

Zero-Knowledge For any possibly malicious efficient $\hat{B}$, there exists an efficient simulator $\operatorname{Sim}_{\hat{B}}$ which on input $f$ outputs a distribution which is computationally indistinguishable from $\operatorname{View}_{\hat{B}}(s, f)$;

\subsubsection{A ZKCSP Protocol}

Given that $s$ is basically the witness of an NP statement, it is possible to construct NIZK proofs of knowledge for it ([104] and the more recent literature on SNARKs [68, 102, 21]). If $V$ is the program that verifies this NIZK proof (using a trusted CRS) then it is easy to implement a ZKCSP over any blockchain with sufficiently rich scripting languages such as Ethereum. The client $B$ will post the transaction 
Transfer $m$ of my coins to a party who publishes a proof $\pi$ such that $V(\pi)=1$

Once $A$ publishes $\pi$ she will get paid and $B$ has confidence that $A$ really knows $s$ (with the simulation and extraction procedures being guaranteed by the simulation and extraction procedure of the NIZK used in the protocol). The question then is how to implement this over more limited scripting languages, including Bitcoin. What follows is a protocol where the program $P$ associated with "payment transactions" can only be of the form "find a SHA256 preimage of a specified value", i.e. hash-locked transactions.

Let $\mathrm{H}$ be a function $\mathrm{H}\{0,1\}^{*} \rightarrow\{0,1\}^{256}$ (i.e. like SHA256). Consider the following function

$$
\mathcal{F}_{f, \mathrm{H}}:\{0,1\}^{*} \times\{0,1\}^{*} \rightarrow\{0,1\}^{256}
$$

defined as follows

$$
\mathcal{F}_{f, \mathrm{H}}(s, r)=\left\{\begin{array}{l}
\mathrm{SHA} 256(r) \text { if } f(s)=1 \\
\mathrm{H}(r) \text { otherwise. }
\end{array}\right.
$$

We are going to use $\mathcal{F}$ to design our new ZKCSP protocol as follows. Informally, the server/seller will choose a random $r$ and send to the client/buyer the value $y=\mathcal{F}_{f, \mathrm{H}}(s, r)$ and proves using a WI protocol that he knows inputs $(s, r)$ such that $y=\mathcal{F}_{f, \mathrm{H}}(s, r)$. Note that if $f(s)=1$ then $y=\operatorname{SHA256}(r)$, otherwise $y=\mathrm{H}(r)$. Moreover, if the output of $\mathrm{H}$ "looks like" the output of SHA256, the client/buyer cannot tell at this point if the server actually knows a "good" $s$ (i.e. $f(s)=1$ ) or not. To detect if this is the case or not should be contingent to a payment by the buyer who therefore publishes the following transaction:

Transfer $m$ Bitcoins to the party who presents a SHA256 preimage of $y$

If $f(s)=1$ then the server/seller knows such a preimage (which is $r$ ), and can publish it to redeem the payment. Moreover, if we assume that finding a SHA256 preimage of $\mathrm{H}(r)$ is hard, then the seller cannot redeem payment when $f(s) \neq 1$.

More formally, let $A$ denote the seller, $B$ denote the buyer and $T$ denote the blockchain:

\section{Protocol 1}

1. $A$ on input $s$, chooses $r$ at random in $\{0,1\}^{256}$ and computes $y=$ $\mathcal{F}_{f, \mathrm{H}}(s, r)$

2. $A$ sends $y$ to $B$ and the two parties engage in a WI proof that the seller knows $r, s$ such that $y=\mathcal{F}_{f, \mathrm{H}}(s, r)$. If the proof fails, the buyer rejects and stops. 
3. $B$ posts a transaction to the Bitcoin blockchain to pay $m$ Bitcoins to the party who presents $x$ such that $\operatorname{SHA256}(x)=y$

4. $A$ presents $z$ to $T$. If SHA256 $(z)=y$ then $T$ posts it and the seller redeems the Bitcoins, otherwise the Bitcoins are returned to the buyer.

We can prove the following 5 :

Theorem 22. Assume that

- SHA256 and $\mathrm{H}$ are a claw-free pair

- the distributions $\operatorname{SHA256}(r)$ and $\mathrm{H}(r)$ for $r$ chosen at random in $\{0,1\}^{256}$ are computationally indistinguishable

then Protocol 1 is a secure ZKCSP protocol.

Proof. (Sketch).

Extraction: Let $\hat{A}$ be an efficient, possibly malicious seller. In step 2, $\hat{A}$ runs a ZK proof of knowledge of the values $s, r$ which can therefore be extracted if the proof is successful. Assume for sake of contradiction that $f(s) \neq 1$ and $\hat{A}$ gets paid. By the correctness of the NIZK we know that since $f(s) \neq 1$, then $y=\mathrm{H}(r)$. In Step $4 \hat{A}$ gets paid only if she produces $z$ such that $y=\operatorname{SHA256}(z)$. Therefore we have found a claw $(r, z)$ for SHA256 and $\mathrm{H}$, since SHA256 $(z)=\mathrm{H}(r)=y$.

Zero-Knowledge is a consequence of the witness indistinguishability of the proof in step 2, and the computational indistinguishability of the output distributions of SHA256 and H. A bit more formally, For step 1, $\operatorname{Sim}_{\hat{B}}$ will choose $r, s$ at random and compute $y=\mathcal{F}_{f, \mathrm{H}}(s, r)$. Note that the message in step 1 is computationally indistinguishable from the message sent by the real $A$ due to the computational indistinguishability of the output distributions of SHA256 and $\mathrm{H}$. For step 2, $\operatorname{Sim}_{\hat{B}}$ will just run a "real" proof that $y=\mathcal{F}_{f, \mathrm{H}}(s, r)$ : note that due to witness indistinguishability, this proof is indistinguishable from a proof of a "correct" proof when the witness is such that $f(s)=1$.

LetTing $B$ Choose the CRS. Note that we only require the proof to be WI. If we were to use a QSP-based SNARK, such as Libsnark, then (as already pointed out in [68]) the verifier $B$ can be allowed to select the CRS, provided the prover $A$ performs some minimal correctness checks (described in detail in Section 5.3.3).

\footnotetext{
5 The assumptions underlying Theorem 22 are expressed in asymptotic terms but for sake of simplicity we are using concrete security parameters and functions used by Bitcoin (e.g. SHA256, with 256 bits output etc). It is easy to reframe the protocol description and the theorem using a security parameter
} 


\subsubsection{An alternative $\mathrm{ZKCP}$ construction}

The idea behind our ZKCSP can be used to build an alternative ZKCP protocol. Recall (using the notation in Section 5.2.5) that in this case, Alice (the seller) wants to sell to Bob (the buyer) a string $f_{A}$ that satisfies some verification procedure $V_{B}$.

The basic idea remains the same: Alice encrypts the string $f_{A}$ with a key $k$ (using any symmetric encryption scheme $E$, i.e. AES) and publishes $\hat{f}=E_{k}\left(f_{A}\right)$ and $y=\operatorname{SHA} 256(k)$. She then proves (using a WI proof) that

$$
y=\mathcal{G}_{V_{B}, \hat{f}, \mathrm{H}}(k)=\left\{\begin{array}{l}
\mathrm{SHA} 256(k) \text { if } V_{B}\left(D_{k}(\hat{f})\right)=1 \\
\mathrm{H}(k) \text { otherwise. }
\end{array}\right.
$$

Note that in this case WI is sufficient since at the end of the protocol, Bob does not know if Alice encrypted a valid string or garbage, and this guarantees that he learns no information about $f_{A}$. At the same time, he is guaranteed that if Alice presents a SHA256 preimage of $y$, then the encrypted string must be valid and he will be able to recover it. Again relying simply on WI, removes the need for a trusted party to generate the CRS, since Bob can be allowed to generate it, provided that Alice performs the minimal checks to guarantee WI (described in Section 5.3.3 and without having to resort to the heavy tests required by subversion-ZK.

\subsubsection{A Protocol with private verification}

In the protocol above we assumed a scenario in which anybody can verify that $s$ is "correct" (i.e. $f(s)=1$ ). There are however situations in which the buyer is the only one who can verify the correctness of $s$. In other words the buyer is only interested in $s$ such that $f(k, s)=1$ where $k$ is a secret "key" held by the buyer. In this case we modify the protocol to have the parties jointly compute the following function

$$
\mathcal{F}_{f, \mathrm{H}}^{\prime}(k, s, r)=\left\{\begin{array}{l}
\mathrm{SHA} 256(r) \text { if } f(k, s)=1 \\
\mathrm{H}(r) \text { otherwise. }
\end{array}\right.
$$

Because both buyer and seller want to keep $k$ and $s$ secret respectively, they will have to use a secure two-party computation protocol, such as Yao's garbled circuit [117] to compute $\mathcal{F}^{\prime}$. It is important to use a two-party computation protocol which is secure against malicious players. The protocol is described below

\section{Protocol 2}

1. $A$ on input $s$, chooses $r$ at random in $\{0,1\}^{256}$ 
2. Using a 2-party computation protocol, secure against malicious players, $A$ and $B$ jointly compute $y=\mathcal{F}_{f, \mathrm{H}}^{\prime}(k, s, r)$ where $k$ is $B$ 's private input.

3. $B$ posts a transaction to the Bitcoin blockchain to pay $m$ Bitcoins to the party who presents $x$ such that SHA256 $(x)=y$

4. $A$ presents $z$ to $T$. If $\operatorname{SHA256}(z)=y$ then $T$ posts it and the seller redeems the Bitcoins, otherwise the Bitcoins are returned to the buyer.

Theorem 23. Assume that

- SHA256 and $\mathrm{H}$ are a claw-free pair

- the distributions SHA256 $(r)$ and $\mathrm{H}(r)$ for $r$ chosen at random in $\{0,1\}^{256}$ are computationally indistinguishable

then Protocol 2 is a secure ZKCSP protocol.

Proof. (Sketch).

Extraction: Let $\hat{A}$ be an efficient, possibly malicious seller. In step $2, \hat{A}$ runs two-party computation protocol which is secure against a malicious adversary. Such protocols require the ability to extract the input during the simulation [93], so we use the simulator of the two-party protocol to extract $r, s$. Now the proof continues as in the proof of Theorem 22. Assume for sake of contradiction tha $f(k, s) \neq 1$ and $\hat{A}$ gets paid. By the correctness of the two-party computation protocol we know that since $f(k, s) \neq 1$, then $y=\mathrm{H}(r)$. In Step $4 \hat{A}$ gets paid only if she produces $z$ such that $y=\operatorname{SHA256}(z)$. Therefore we have found a claw $(r, z)$ for SHA256 and $\mathrm{H}$, since $\operatorname{SHA} 256(z)=\mathrm{H}(r)=y$.

Zero-Knowledge is a consequence of the simulatability of the two-party protocol in step 2, and the computational indistinguishability of the output distributions of SHA256 and H. A bit more formally, For steps 1 and $2, \operatorname{Sim}_{\hat{B}}$ will choose $r$ at random and compute $y=$ SHA256 $(r)$ and simulate the two-party computation with $y$ as output. Now if $A$ has a correct $s$ then step 4 will be executed and the simulator will simulate it perfectly by releasing a SHA256 preimage of $y$. If $A$ did not have a correct $s$, then step 4 is a message between $A$ and $T$ but is not posted to the blockchain and therefore does not belong to the view of $\hat{B}$ and the simulator does not have to simulate it.

\subsection{Implementation}

In this section we discuss our implementation work on: the attack against Maxwell' s ZKCP; a proof of concept of our protocol for ZKCP for Services; a more efficient SHA256 circuit implementation (used in Protocol 2). The code is available 
at [41, 42]. All benchmarks in this section were evaluated on a Debian 3.16.391 x86_64 GNU/Linux Virtual Machine (virtual CPU and RAM respectively 2.4 GhZ and 3.5 GB).

\subsubsection{Pay-to-Sudoku}

Attack. We modified the Pay-to-Sudoku's code [36] in a way that allows a malicious buyer to learn information about the value of a cell of the Sudoku solution without paying for it. To do that we created a modified version of libsnark that implements the attack (under "Setting all the $p k$ equal to the identity except for one wire"). The malicious buyer can generate a CRS running this code and find out the exact value of a cell with probability at least $1 / 9$ from the proof received by the seller. Note that the seller in [36] does not find out the CRS was generated maliciously and that we did not modify any code involving the Sudoku solution seller or the prover in libsnark.

Alternative Pay-to-Sudoku. We also implemented our alternative ZKCP protocol using only WI proofs (described in Section 5.4.3) for the case of Pay-toSudoku. In our protocol the prover runs a bit slower than the insecure original protocol due to the fact that the proof is run over a larger circuit (verification time is basically unchanged as to be expected in the case of QSP-based protocols). On the other hand, the cost of the expensive subversion-ZK CRS checks to the original Pay-to-Sudoku protocol dominates the overhead of the larger circuit in our protocol (which does not require such expensive checks). In particular, our results suggest that the proving process would require more than an hour in total (instead of a few seconds without the ZK-subversion checks). This time has been obtained by computing $t_{P} \cdot n_{P}$, where $t_{P}$ is the experimental estimate for the the average time per pairing check (i.e. $4.50 \mathrm{~ms}$ ) and $n_{P}$ is the number of pairing checks for subversion-ZK in [65]. A lower bound on $n_{P}$ is $7 m$ where $m$ is the number of constraints. The quantity $m$ is sligthly greater than $115 \mathrm{~K}$ for Payto-Sudoku. In these benchmarks we used curve ALT_BN128, the same originally used in Pay-to-Sudoku. Table 5.1 summarizes the performance comparison.

\subsubsection{Proofs of Retrievability (PoR) over Bitcoin}

As a proof of concept, we provide an implementation for a ZKCSP for Auditing of Proofs of Retrievability (PoR). Our implementation is based on the PoR scheme in [108] (See Appendix for details of the the scheme). In the context of PoR, a party delegates storage of her data to a server. A PoR scheme consists of an (efficient) protocol by which the delegator can verify at any time whether the server is still keeping her data intact. Our protocol allows the client to pay the server if such verification procedure succeeds. The PoR scheme in [108] can be instantiated both as privately and publicly verifiable (see appendix for details). 
Chapter 5. Extended Fair Exchange Over the Bitcoin Blockchain: Zero

Knowledge Contingent Payments for Services

\begin{tabular}{|c|c|c|}
\hline & $\begin{array}{l}\text { ZKCSP } \\
\text { for Su- } \\
\text { doku } \\
\text { with WI } \\
\text { checks }\end{array}$ & $\begin{array}{l}\text { Pay-to- } \\
\text { Sudoku } \\
\text { with } \\
\text { Subversion- } \\
\text { ZK }\end{array}$ \\
\hline Key Generation & $54 \mathrm{~s}$ & $22 \mathrm{~s}$ \\
\hline Proof & $10900 \mathrm{~ms}$ & $\begin{array}{l}>1 \text { hour } \\
(5500 \mathrm{~ms} \\
\text { without } \\
\text { checks })\end{array}$ \\
\hline Verification & $25 \mathrm{~ms}$ & $24 \mathrm{~ms}$ \\
\hline
\end{tabular}

Table 5.1: Estimated Running Time for Contingent Payment for Sudoku with checked $C R S$

\begin{tabular}{l|l|l} 
& Bandwidth $\mathbf{( K B )}$ & Time $(\mathbf{m s})$ \\
\hline Garbler & 38879 & 155 \\
\hline Evaluator & 51 & 159
\end{tabular}

Table 5.2: Stats for Fair Auditing of Privately Verifiable PoR with Secure Two Party Computation.

For this application, the curve we used in libsnark was MNT6. Although less efficient than BN128 or ALT_BN128, this curve was one of the few ones which offered verification gadgets for pairings.

Private Verification. In this case the PoR scheme in [108] reduces to the verification of a (linearly homomorphic) MAC jointly by the server and the client. Here the PoR is successful if the server proves to the client that it knows $s=(m, t)$ such that $t=M A C_{k}(m)$ where $k$ is the secret authentication key of the client.

We used Protocol 2 described in Section 5.4.4 where $f(k, s)=1$ if and only if $s=(m, t)$ and $t=M A C_{k}(m)$. We implemented a two-party protocol for the computation of the associated function $\mathcal{F}^{\prime}$ using the SCAPI library [58] following [112. We used $\lambda=128$ bits of computational security and $\rho=80$ bits of statistical security. We chose a Carter-Wegman [114] style MAC, specifically the one in [88]. The circuit has 150441 gates and 151017 wires. The number of input wires for the two parties, seller and buyer, are respectively 416 and 160. The output of the circuit is 256 bits. See Table 5.2 for evaluation of running time and bandwidth.

Public Verification. In this case the PoR scheme in 108 reduces to the verification of a (linearly homomorphic) signature scheme, specifically the BLS scheme from [34]. More specifically the PoR is succesfull if the server proves to the client that it knows $s=(m, \sigma)$ such that $\operatorname{Ver}(P K, m, \sigma)=1$ where $\operatorname{Ver}$ is 
Chapter 5. Extended Fair Exchange Over the Bitcoin Blockchain: Zero

Knowledge Contingent Payments for Services

\begin{tabular}{l|l|l} 
& Bandwidth Time $(\mathbf{m s})$ \\
\hline Key Generation & $\begin{array}{l}p k: 41959 \\
\text { KB } \\
s k: 13 \mathrm{~KB}\end{array}$ & \\
\hline Proof & 374 bytes & 3287 \\
\hline Verification & - & 37
\end{tabular}

Table 5.3: Stats for Fair Auditing of Publicly Verifiable PoR with SNARKs.

the verification algorithm of the BLS signature scheme, and $P K$ is the public key of the client.

In this case we used Protocol 1 described in Section 5.4.2 where $f(s)=1$ if and only if $s=(m, \sigma)$ and $\operatorname{Ver}(P K, m, \sigma)=1$. We implemented ZK-SNARK to enable the server to prove that she knows $(s, r)$ such that $y=\mathcal{F}_{f, \mathrm{H}}(s, r)$. This proof was implemented in $\mathrm{C}++$ using libsnark [21]. The function $\mathcal{F}$ was described in libsnark as set of constraints called Rank-One Constraint System (R1CS). Implementing the above $\mathcal{F}$ we obtained a R1CS system with 39409 constraints. In this setting we used $\lambda=80$ bits of computational security. See Table 5.3 for evaluation of running time and bandwidth.

For

Generation of the CRS The timing results in the tables above refer to a Key Generation performed by a trusted party.

\subsubsection{A More Efficient SHA256 Circuit Implementation}

SCAPI and other cryptographic libraries require the user to supply the circuit for the function that want to compute. Building a circuit file in this format is complex, and there is a library of such circuit files made available by researchers at Bristol University [109].

As part of the implementation in the proof of concept above, we constructed a new optimized reusable boolean circuit for SHA256. Our circuit may be of independent use for circuit-based MPC and FHE protocols that require SHA256 computations.

To the best of our knowledge, the only other re-usable circuit implementation openly available for SHA256 was developed the Bristol circuit. See Table 5.4 for a comparison of the circuit parameters between the Bristol circuit and ours. Our circuit compares favorably both with respect to the total number of gates and to the number of AND gates. The latter parameter is particularly important if one intends to use SHA256 in Secure Multi-Party Computation. In fact, in modern MPC protocols the number of AND gates dominates the total evaluation cost thanks to a technique called Free-XOR [87] which evaluates XOR gates 


\begin{tabular}{l|l|l} 
& Bristol Circuit & Our Circuit \\
\hline Total gates & 236112 & $\mathbf{1 1 6 2 4 5}$ \\
\hline AND gates & 90825 & $\mathbf{2 2 2 7 2}$ \\
\hline XOR gates & $\mathbf{4 2 0 2 9}$ & 91780 \\
\hline INV gates & 103258 & $\mathbf{2 1 9 4}$
\end{tabular}

Table 5.4: Number of gates in SHA256 circuit implementations.

"for free". In the process of building our SHA256 circuit we developed a library for semi-automated generation of optimized boolean circuits which we believe may be of independent interest. We stress that our contribution here is not the optimizations themselves as they were mostly straightforward from the SHA2 specification, but or contributions is the the optimized implementation of SHA2 in a boolean circuit format that can be reused by other cryptographic libraries and protocols. 


\section{Conclusions}

In this thesis we addressed the question of how different entities can be forced to behave honestly when interacting over the Internet. In particular, we advanced the state of the art in the two important paradigms of Cloud computing and E-commerce.

Authenticity of Computations Outsourced to the Cloud. With respect to Cloud computing, we focused on the problem of granting authenticity of computations which are outsourced to the Cloud via homomorphic authenticators. To this aim, we advanced the state of the art with respect to three different aspects: definitions, efficiency and functionalities.

In terms of definitions, we defined a new security model which is stronger and simpler when compared with the state of the art one. Moreover, we provided two different compilers that allow for transforming any homomorphic signature scheme which is secure in a weaker model (the so called semi-adaptive) into one which supports the same class of functions and is secure in our new stronger model.

Considering efficiency, we focused on the problem of reducing verification key size for homomorphic signature schemes which are proven secure in the standard model. As a result, we came up with a new cryptographic tool, called Asymmetric Programmable Hash Functions (APHFs, for short), that, while being of independent interest, allows for linearly homomorphic signature schemes with verification key size sublinear (rather than linear) in the dataset size.

Regarding functionalities, we addressed the problem of extending the concept of homomorphic authenticators to functions that are evaluated over inputs authenticated using different secret keys: this kind of property is particularly useful in several real life scenarios and was never considered before. Moreover, we presented the first concrete constructions of multi-key homomorphic signatures (publicly verifiable) and multi-key message authentication codes (privately verifiable).

While recognizing the value of our contributions, we think it is also worth to 
focus on the variety of problems that are still open and could represent interesting and challenging future research directions in these research area.

For what regards homomorphic authenticators, we consider this thesis as contributing to a better understanding of this powerful cryptographic tool, since the results we obtained are, in a sense, first steps in new research lines.

Regarding efficiency, and in particular the reduction of the verification key size in homomorphic signatures, before this thesis it was not even clear if it would have been possible to go beyond the linear dependance with respect to the dataset size in the standard model: concretely, the constant size of verification keys in the random oracle model seemed to be inherent to its peculiar properties, which are not achievable in the standard model. That is the reason why coming up with a new cryptographic tool which mimic random oracle functionalities (and, in particular, its programmability) was crucial to obtain signatures with sublinear verification keys. Nevertheless, even if our result represents a remarkable step in showing the possibility of sub-linear verification keys, it leaves some open questions: first, it is unknown if a similar result can be obtained in the case of homomorphic signatures for arbitrary functions (and not only for linear ones); second, it remains open if our result can be improved by obtaining verification keys that depend logarithmically (or even constantly, as in the random oracle model) on the dataset size.

In terms of functionalities, we showed that it is actually possible to define and have concrete constructions of homomorphic authenticators which deal with multiple keys. We stress that this notion had not been considered before, and as we show in this thesis, this new feature allows for new applications in real life scenarios.

Of course, the work in this new setting has just started: an important problem, which is still open, is if it is possible to get multi-key homomorphic authenticators whose size does not depend linearly on the number of users who provided input to the authenticated computation. This answer has been answered affirmatively by Lai et al. [91] who show a scheme with signatures of fixed size. However, their result, unlike ours, make use of non standard, non-falsifiable assumptions. It therefore remains an intriguing open question if such a result can be achieved under standard assumptions. We believe that achieving the aforementioned goals will require new, unknown cryptographic techniques, and this fact somehow tells us that homomorphic authenticators are a powerful cryptographic tool that we still have to deeply understand.

Fair Exchange Over the Bitcoin Blockchain. In the second part of this thesis we considered a different paradigm, the one of E-commerce. In particular, we focused on the problem of fair exchange using the Bitcoin Blockchain as a trusted party. Here our contribution if twofold, and addresses security and functionalities of the state of the art protocol which allows for buying and selling digital goods over the Bitcoin Blockchain: the so called "Zero Knowledge Contingent Payments 
(ZKCP)". We found a flaw in ZKCP and we proposed a new protocol that on one hand fixes this flaw efficiently, while on the other hand extends the original functionalities of ZKCP allowing to deal with the so called "services".

The results of these thesis represents an additional step towards the use of Bitcoin beyond being a system which allows for transferring value without the need of a central authority, but of course there are interesting problems that remain open. First of all, focusing on the contingent payments primitive, it would be nice to have a protocol which is publicly verifiable. Indeed in our protocol, as it is now, a third party would not be able to verify that buyer and seller were not colluding during the protocol. This aspect becomes pivotal in settings like auctions where a bidder wants to be sure that a seller is not colluding with another bidder in order to cheat the others. Said in another way, our protocol works fine in a scenario where buyer and seller do not trust each other and are not colluding against somebody else, but can not deal with more generic settings. Another interesting open problem is if it is possible to build zero knowledge proof systems which have the same efficiency as the ones in [68] with a setup which does not need to be trusted and which does not depend on the circuit being evaluated. Some advancements in this sense have been recently made by Bünz et al. 39] that proposed Bulletproofs, a zero knowledge proof systems which relies on the common random string model rather than in the common reference string one. On the positive side, in this scheme the setup does not need to be trusted, and it is independent with respect to the circuit being evaluated; on the negative side, Bulletproofs verification complexity is linear in the size of the circuit being evaluated, leading to a less efficient verification procedure when comparing with [68]. 


\section{Bibliography}

[1] B. Abdolmaleki, K. Baghery, H. Lipmaa, and M. Zajac. A subversionresistant snark. Cryptology ePrint Archive, Report 2017/599, 2017. http: //eprint.iacr.org/2017/599.

[2] M. Abe, J. Groth, M. Ohkubo, and M. Tibouchi. Structure-preserving signatures from type II pairings. In J. A. Garay and R. Gennaro, editors, CRYPTO 2014, Part I, volume 8616 of LNCS, pages 390-407. Springer, Aug. 2014.

[3] S. Agrawal and D. Boneh. Homomorphic MACs: MAC-based integrity for network coding. In M. Abdalla, D. Pointcheval, P.-A. Fouque, and D. Vergnaud, editors, ACNS 09, volume 5536 of LNCS, pages 292-305. Springer, June 2009.

[4] S. Agrawal, D. Boneh, X. Boyen, and D. M. Freeman. Preventing pollution attacks in multi-source network coding. In P. Q. Nguyen and D. Pointcheval, editors, PKC 2010, volume 6056 of LNCS, pages 161-176. Springer, May 2010 .

[5] M. Ajtai. Generating hard instances of lattice problems (extended abstract). In 28th ACM STOC, pages 99-108. ACM Press, May 1996.

[6] M. Ajtai. Generating hard instances of the short basis problem. In $A u$ tomata, Languages and Programming, pages 1-9. Springer, 1999.

[7] J. Alwen and C. Peikert. Generating shorter bases for hard random lattices. Theory of Computing Systems, 48(3):535-553, 2011.

[8] G. Andresen. Github: Proposal: open up IsStandard for P2SH transactions. https://gist.github.com/gavinandresen/88be40c141bc67acb247, 2017. 
[9] M. Andrychowicz, S. Dziembowski, D. Malinowski, and L. Mazurek. Fair two-party computations via bitcoin deposits. In R. Böhme, M. Brenner, T. Moore, and M. Smith, editors, FC 2014 Workshops, volume 8438 of LNCS, pages 105-121. Springer, Mar. 2014.

[10] M. Andrychowicz, S. Dziembowski, D. Malinowski, and L. Mazurek. On the malleability of bitcoin transactions. In International Conference on Financial Cryptography and Data Security, pages 1-18. Springer, 2015.

[11] N. Asokan, V. Shoup, and M. Waidner. Optimistic fair exchange of digital signatures (extended abstract). In K. Nyberg, editor, EUROCRYPT'98, volume 1403 of LNCS, pages 591-606. Springer, May / June 1998.

[12] N. Attrapadung and B. Libert. Homomorphic network coding signatures in the standard model. In D. Catalano, N. Fazio, R. Gennaro, and A. Nicolosi, editors, PKC 2011, volume 6571 of LNCS, pages 17-34. Springer, Mar. 2011.

[13] N. Attrapadung, B. Libert, and T. Peters. Computing on authenticated data: New privacy definitions and constructions. In X. Wang and K. Sako, editors, ASIACRYPT 2012, volume 7658 of LNCS, pages 367-385. Springer, Dec. 2012.

[14] N. Attrapadung, B. Libert, and T. Peters. Efficient completely contexthiding quotable and linearly homomorphic signatures. In K. Kurosawa and G. Hanaoka, editors, PKC 2013, volume 7778 of LNCS, pages 386-404. Springer, Feb. / Mar. 2013.

[15] M. Backes, D. Fiore, and R. M. Reischuk. Verifiable delegation of computation on outsourced data. In A.-R. Sadeghi, V. D. Gligor, and M. Yung, editors, ACM CCS 13, pages 863-874. ACM Press, Nov. 2013.

[16] W. Banasik, S. Dziembowski, and D. Malinowski. Efficient zero-knowledge contingent payments in cryptocurrencies without scripts. In European Symposium on Research in Computer Security, pages 261-280. Springer, 2016.

[17] M. Bellare, G. Fuchsbauer, and A. Scafuro. Nizks with an untrusted crs: security in the face of parameter subversion. In Advances in Cryptology - ASIACRYPT 2016 - 22nd International Conference on the Theory and Application of Cryptology and Information Security, Part II, pages 777-804. Springer, 2016.

[18] E. Ben-Sasson, A. Chiesa, D. Genkin, S. Kfir, E. Tromer, M. S. L. Virza, and others external contributors. Libsnark, 2017. https://github.com/ scipr-lab/libsnark. 
[19] E. Ben-Sasson, A. Chiesa, M. Green, E. Tromer, and M. Virza. Secure sampling of public parameters for succinct zero knowledge proofs. In IEEE Security and Privacy Conference, pages 287-304, 2015.

[20] E. Ben-Sasson, A. Chiesa, E. Tromer, and M. Virza. Scalable zero knowledge via cycles of elliptic curves. In J. A. Garay and R. Gennaro, editors, CRYPTO 2014, Part II, volume 8617 of LNCS, pages 276-294. Springer, Aug. 2014.

[21] E. Ben-Sasson, A. Chiesa, E. Tromer, and M. Virza. Succinct noninteractive zero knowledge for a von neumann architecture. In Proceedings of the 23rd USENIX Conference on Security Symposium, SEC'14, pages 781-796, Berkeley, CA, USA, 2014. USENIX Association.

[22] S. Benabbas, R. Gennaro, and Y. Vahlis. Verifiable delegation of computation over large datasets. In P. Rogaway, editor, CRYPTO 2011, volume 6841 of LNCS, pages 111-131. Springer, Aug. 2011.

[23] N. Bitansky, R. Canetti, A. Chiesa, and E. Tromer. Recursive composition and bootstrapping for SNARKS and proof-carrying data. In D. Boneh, T. Roughgarden, and J. Feigenbaum, editors, 45th ACM STOC, pages 111120. ACM Press, June 2013.

[24] BitcoinWiki. Bitcoin transaction, 2016. https://en.bitcoin.it/wiki/ Transaction.

[25] BitcoinWiki. Zero knowledge contingent payment, 2016. https://en. bitcoin.it/wiki/Zero_Knowledge_Contingent_Payment.

[26] BitcoinWiki. Scripts, 2017. https://en.bitcoin.it/wiki/Script.

[27] D. Boneh and X. Boyen. Efficient selective-ID secure identity based encryption without random oracles. In C. Cachin and J. Camenisch, editors, EUROCRYPT 2004, volume 3027 of LNCS, pages 223-238. Springer, May 2004.

[28] D. Boneh and X. Boyen. Short signatures without random oracles. In C. Cachin and J. Camenisch, editors, EUROCRYPT 2004, volume 3027 of LNCS, pages 56-73. Springer, May 2004.

[29] D. Boneh and M. K. Franklin. Identity-based encryption from the Weil pairing. In J. Kilian, editor, CRYPTO 2001, volume 2139 of LNCS, pages 213-229. Springer, Aug. 2001.

[30] D. Boneh, D. Freeman, J. Katz, and B. Waters. Signing a linear subspace: Signature schemes for network coding. In S. Jarecki and G. Tsudik, editors, PKC 2009, volume 5443 of LNCS, pages 68-87. Springer, Mar. 2009. 
[31] D. Boneh and D. M. Freeman. Homomorphic signatures for polynomial functions. In K. G. Paterson, editor, EUROCRYPT 2011, volume 6632 of LNCS, pages 149-168. Springer, May 2011.

[32] D. Boneh and D. M. Freeman. Linearly homomorphic signatures over binary fields and new tools for lattice-based signatures. In D. Catalano, N. Fazio, R. Gennaro, and A. Nicolosi, editors, PKC 2011, volume 6571 of LNCS, pages 1-16. Springer, Mar. 2011.

[33] D. Boneh, C. Gentry, S. Gorbunov, S. Halevi, V. Nikolaenko, G. Segev, V. Vaikuntanathan, and D. Vinayagamurthy. Fully key-homomorphic encryption, arithmetic circuit $\mathrm{ABE}$ and compact garbled circuits. In P. Q. Nguyen and E. Oswald, editors, EUROCRYPT 2014, volume 8441 of LNCS, pages 533-556. Springer, May 2014.

[34] D. Boneh, B. Lynn, and H. Shacham. Short signatures from the weil pairing. In International Conference on the Theory and Application of Cryptology and Information Security, pages 514-532. Springer, 2001.

[35] J. Bonneau, A. Miller, J. Clark, A. Narayanan, J. A. Kroll, and E. W. Felten. Sok: Research perspectives and challenges for bitcoin and cryptocurrencies, 2015.

[36] S. Bowe. pay-to-sudoku, 2016. https://github.com/zcash/ pay-to-sudoku.

[37] S. Bowe, A. Gabizon, and M. Green. A multi-party protocol for constructing the public parameters of the pinocchio zk-snark. 2016. https://github. $\mathrm{com} / \mathrm{zcash} / \mathrm{mpc} / \mathrm{blob} / \mathrm{master} /$ whitepaper.pdf.

[38] X. Boyen, X. Fan, and E. Shi. Adaptively secure fully homomorphic signatures based on lattices. Cryptology ePrint Archive, Report 2014/916, 2014. http://eprint.iacr.org/2014/916.

[39] B. Bünz, J. Bootle, D. Boneh, A. Poelstra, P. Wuille, and G. Maxwell. Bulletproofs: Efficient range proofs for confidential transactions. Technical report.

[40] V. Buterin et al. A next-generation smart contract and decentralized application platform, 2014.

[41] M. Campanelli, R. Gennaro, S. Goldfeder, and L. Nizzardo. An attack to pay-to-sudoku. https://github.com/matteocam/ pay-to-sudoku-attack, 2017.

[42] M. Campanelli, R. Gennaro, S. Goldfeder, and L. Nizzardo. Zkcsp over bitcoin. https://github.com/matteocam/zkcsp-over-bitcoin, 2017. 
[43] D. Catalano and D. Fiore. Practical homomorphic MACs for arithmetic circuits. In T. Johansson and P. Q. Nguyen, editors, EUROCRYPT 2013, volume 7881 of LNCS, pages 336-352. Springer, May 2013.

[44] D. Catalano, D. Fiore, R. Gennaro, and L. Nizzardo. Generalizing homomorphic MACs for arithmetic circuits. In H. Krawczyk, editor, PKC 2014, volume 8383 of $L N C S$, pages 538-555. Springer, Mar. 2014.

[45] D. Catalano, D. Fiore, R. Gennaro, and K. Vamvourellis. Algebraic (trapdoor) one-way functions and their applications. In A. Sahai, editor, TCC 2013, volume 7785 of LNCS, pages 680-699. Springer, Mar. 2013.

[46] D. Catalano, D. Fiore, and L. Nizzardo. Programmable hash functions go private: Constructions and applications to (homomorphic) signatures with shorter public keys. In CRYPTO 2015. Springer, 2015. Full version at: https://eprint.iacr.org/2015/826.

[47] D. Catalano, D. Fiore, and B. Warinschi. Adaptive pseudo-free groups and applications. In K. G. Paterson, editor, EUROCRYPT 2011, volume 6632 of $L N C S$, pages 207-223. Springer, May 2011.

[48] D. Catalano, D. Fiore, and B. Warinschi. Efficient network coding signatures in the standard model. In M. Fischlin, J. Buchmann, and M. Manulis, editors, PKC 2012, volume 7293 of $L N C S$, pages 680-696. Springer, May 2012 .

[49] D. Catalano, D. Fiore, and B. Warinschi. Homomorphic signatures with efficient verification for polynomial functions. In J. A. Garay and R. Gennaro, editors, CRYPTO 2014, Part I, volume 8616 of LNCS, pages 371-389. Springer, Aug. 2014.

[50] M. Chase, M. Kohlweiss, A. Lysyanskaya, and S. Meiklejohn. Malleable signatures: New definitions and delegatable anonymous credentials. In 2014 IEEE 27th Computer Security Foundations Symposium, pages 199213. IEEE, 2014.

[51] S. G. Choi, J. Katz, R. Kumaresan, and C. Cid. Multi-client non-interactive verifiable computation. In A. Sahai, editor, TCC 2013, volume 7785 of LNCS, pages 499-518. Springer, Mar. 2013.

[52] K.-M. Chung, Y. Kalai, and S. P. Vadhan. Improved delegation of computation using fully homomorphic encryption. In T. Rabin, editor, CRYPTO 2010, volume 6223 of LNCS, pages 483-501. Springer, Aug. 2010.

[53] R. Cleve. Limits on the security of coin flips when half the processors are faulty (extended abstract). In J. Hartmanis, editor, Proceedings of the 
18th Annual ACM Symposium on Theory of Computing, May 28-30, 1986, Berkeley, California, USA, pages 364-369. ACM, 1986.

[54] G. G. Dagher, B. Bünz, J. Bonneau, J. Clark, and D. Boneh. Provisions: Privacy-preserving proofs of solvency for bitcoin exchanges. In Proceedings of the 22nd ACM SIGSAC Conference on Computer and Communications Security, pages 720-731. ACM, 2015.

[55] G. Danezis, C. Fournet, J. Groth, and M. Kohlweiss. Square span programs with applications to succinct NIZK arguments. In P. Sarkar and T. Iwata, editors, ASIACRYPT 2014, Part I, volume 8873 of LNCS, pages 532-550. Springer, Dec. 2014.

[56] Y. Desmedt. Computer security by redefining what a computer is. NSPW, 1993.

[57] Y. Dodis, R. Ostrovsky, L. Reyzin, and A. Smith. Fuzzy extractors: How to generate strong keys from biometrics and other noisy data. SIAM Journal on computing, 38(1):97-139, 2008.

[58] Y. Ejgenberg, M. Farbstein, M. Levy, and Y. Lindell. Scapi: The secure computation application programming interface. IACR Cryptology EPrint Archive, 2012:629, 2012.

[59] K. Elkhiyaoui, M. Önen, and R. Molva. Online-offline homomorphic signatures for polynomial functions. Cryptology ePrint Archive, Report 2015/954, 2015. http://eprint.iacr.org/.

[60] P. Erdös, P. Frankel, and Z. Furedi. Families of finite sets in which no set is covered by the union of $r$ others. Israeli Journal of Mathematics, 51:79-89, 1985.

[61] U. Feige and A. Shamir. Witness indistinguishable and witness hiding protocols. In Proceedings of the 22nd Annual ACM Symposium on Theory of Computing, May 13-17, 1990, Baltimore, Maryland, USA, pages 416426, 1990.

[62] D. Fiore and R. Gennaro. Publicly verifiable delegation of large polynomials and matrix computations, with applications. In T. Yu, G. Danezis, and V. D. Gligor, editors, ACM CCS 12, pages 501-512. ACM Press, Oct. 2012 .

[63] D. M. Freeman. Improved security for linearly homomorphic signatures: A generic framework. In M. Fischlin, J. Buchmann, and M. Manulis, editors, PKC 2012, volume 7293 of $L N C S$, pages 697-714. Springer, May 2012. 
[64] E. S. V. Freire, D. Hofheinz, K. G. Paterson, and C. Striecks. Programmable hash functions in the multilinear setting. In R. Canetti and J. A. Garay, editors, CRYPTO 2013, Part I, volume 8042 of LNCS, pages 513-530. Springer, Aug. 2013.

[65] G. Fuchsbauer. Subversion-zero-knowledge snarks. Cryptology ePrint Archive, Report 2017/587, 2017. http://eprint.iacr.org/2017/587.

[66] K. O. Geddes, S. R. Czapor, and G. Labahn. Algorithms for Computer Algebra. Kluwer Academic Publishers, Norwell, MA, USA, 1992.

[67] R. Gennaro, C. Gentry, and B. Parno. Non-interactive verifiable computing: Outsourcing computation to untrusted workers. In T. Rabin, editor, CRYPTO 2010, volume 6223 of LNCS, pages 465-482. Springer, Aug. 2010.

[68] R. Gennaro, C. Gentry, B. Parno, and M. Raykova. Quadratic span programs and succinct NIZKs without PCPs. In T. Johansson and P. Q. Nguyen, editors, EUROCRYPT 2013, volume 7881 of LNCS, pages 626645. Springer, May 2013.

[69] R. Gennaro, J. Katz, H. Krawczyk, and T. Rabin. Secure network coding over the integers. In P. Q. Nguyen and D. Pointcheval, editors, PKC 2010, volume 6056 of LNCS, pages 142-160. Springer, May 2010.

[70] R. Gennaro and D. Wichs. Fully homomorphic message authenticators. In K. Sako and P. Sarkar, editors, ASIACRYPT 2013, Part II, volume 8270 of $L N C S$, pages 301-320. Springer, Dec. 2013.

[71] C. Gentry. Fully homomorphic encryption using ideal lattices. In M. Mitzenmacher, editor, 41st ACM STOC, pages 169-178. ACM Press, May / June 2009.

[72] C. Gentry, C. Peikert, and V. Vaikuntanathan. Trapdoors for hard lattices and new cryptographic constructions. In R. E. Ladner and C. Dwork, editors, 40th ACM STOC, pages 197-206. ACM Press, May 2008.

[73] O. Goldreich and H. Krawczyk. On the composition of zero-knowledge proof systems. SIAM J. Comput., 25(1):169-192, 1996.

[74] O. Goldreich and Y. Oren. Definitions and properties of zero-knowledge proof systems. J. Cryptology, 7(1):1-32, 1994.

[75] S. Goldwasser, Y. T. Kalai, and G. N. Rothblum. Delegating computation: interactive proofs for muggles. In R. E. Ladner and C. Dwork, editors, 40th ACM STOC, pages 113-122. ACM Press, May 2008. 
[76] S. Goldwasser, S. Micali, and R. L. Rivest. A digital signature scheme secure against adaptive chosen-message attacks. SIAM J. Comput., 17(2):281-308, 1988.

[77] S. Gorbunov, V. Vaikuntanathan, and D. Wichs. Leveled fully homomorphic signatures from standard lattices. In Proceedings of the Forty-Seventh Annual ACM on Symposium on Theory of Computing, STOC 2015, pages 469-477.

[78] S. D. Gordon, J. Katz, F.-H. Liu, E. Shi, and H.-S. Zhou. Multi-client verifiable computation with stronger security guarantees. LNCS, pages 144168. Springer, 2015.

[79] M. Green and S. Hohenberger. Practical adaptive oblivious transfer from simple assumptions. In Y. Ishai, editor, TCC 2011, volume 6597 of LNCS, pages 347-363. Springer, Mar. 2011.

[80] G. Hanaoka, T. Matsuda, and J. C. N. Schuldt. On the impossibility of constructing efficient key encapsulation and programmable hash functions in prime order groups. In R. Safavi-Naini and R. Canetti, editors, CRYPTO 2012, volume 7417 of LNCS, pages 812-831. Springer, Aug. 2012.

[81] K. Haralambiev, T. Jager, E. Kiltz, and V. Shoup. Simple and efficient public-key encryption from computational Diffie-Hellman in the standard model. In P. Q. Nguyen and D. Pointcheval, editors, PKC 2010, volume 6056 of $L N C S$, pages 1-18. Springer, May 2010.

[82] D. Hofheinz, T. Jager, and E. Kiltz. Short signatures from weaker assumptions. In D. H. Lee and X. Wang, editors, ASIACRYPT 2011, volume 7073 of $L N C S$, pages 647-666. Springer, Dec. 2011.

[83] D. Hofheinz and E. Kiltz. Programmable hash functions and their applications. In D. Wagner, editor, CRYPTO 2008, volume 5157 of $L N C S$, pages 21-38. Springer, Aug. 2008.

[84] D. Hofheinz and E. Kiltz. Programmable hash functions and their applications. Journal of Cryptology, 25(3):484-527, July 2012.

[85] R. Johnson, D. Molnar, D. X. Song, and D. Wagner. Homomorphic signature schemes. In B. Preneel, editor, CT-RSA 2002, volume 2271 of $L N C S$, pages 244-262. Springer, Feb. 2002.

[86] A. Juels and B. S. Kaliski Jr. Pors: Proofs of retrievability for large files. In Proceedings of the 14th ACM conference on Computer and communications security, pages 584-597. Acm, 2007. 
[87] V. Kolesnikov and T. Schneider. Improved garbled circuit: Free xor gates and applications. Automata, Languages and Programming, pages 486-498, 2008.

[88] H. Krawczyk. Lfsr-based hashing and authentication. In Advances in Cryptology - CRYPTO '94, 14th Annual International Cryptology Conference, Santa Barbara, California, USA, August 21-25, 1994, Proceedings, volume 839, pages 129-139. Springer, 1994.

[89] R. Kumar, S. Rajagopalan, and A. Sahai. Coding constructions for blacklisting problems without computational assumptions. In M. J. Wiener, editor, CRYPTO'99, volume 1666 of LNCS, pages 609-623. Springer, Aug. 1999.

[90] A. Küpçü and A. Lysyanskaya. Usable optimistic fair exchange. In J. Pieprzyk, editor, CT-RSA 2010, volume 5985 of LNCS, pages 252-267. Springer, Mar. 2010.

[91] R. W. Lai, R. K. Tai, H. W. Wong, and S. S. Chow. Multi-key homomorphic signatures unforgeable under insider corruption. Technical report.

[92] B. Libert, T. Peters, M. Joye, and M. Yung. Linearly homomorphic structure-preserving signatures and their applications. In R. Canetti and J. A. Garay, editors, CRYPTO 2013, Part II, volume 8043 of LNCS, pages 289-307. Springer, Aug. 2013.

[93] Y. Lindell and B. Pinkas. An efficient protocol for secure two-party computation in the presence of malicious adversaries. In Annual International Conference on the Theory and Applications of Cryptographic Techniques, pages 52-78. Springer, 2007.

[94] G. Maxwell. Zero knowledge contingent payment, 2015. https://en. bitcoin.it/wiki/Zero_Knowledge_Contingent_Payment.

[95] D. Micciancio. Almost perfect lattices, the covering radius problem, and applications to Ajtai's connection factor. SIAM Journal on Computing, 34(1):118-169, 2004. Preliminary version in STOC 2002.

[96] D. Micciancio and C. Peikert. Trapdoors for lattices: Simpler, tighter, faster, smaller. In D. Pointcheval and T. Johansson, editors, EUROCRYPT 2012, volume 7237 of LNCS, pages 700-718. Springer, Apr. 2012.

[97] D. Micciancio and C. Peikert. Hardness of SIS and LWE with small parameters. In R. Canetti and J. A. Garay, editors, CRYPTO 2013, Part I, volume 8042 of LNCS, pages 21-39. Springer, Aug. 2013. 
[98] D. Micciancio and O. Regev. Worst-case to average-case reductions based on Gaussian measures. In 45th FOCS, pages 372-381. IEEE Computer Society Press, Oct. 2004.

[99] S. Mitsunari, R. Saka, and M. Kasahara. A new traitor tracing. IEICE Transactions, E85-A(2):481-484, Feb. 2002.

[100] S. Nakamoto. Bitcoin: A peer-to-peer electronic cash system. 2008.

[101] A. Narayanan, J. Bonneau, E. Felten, A. Miller, and S. Goldfeder. Bitcoin and Cryptocurrency Technologies: A Comprehensive Introduction. Princeton University Press, 2016.

[102] B. Parno, J. Howell, C. Gentry, and M. Raykova. Pinocchio: Nearly practical verifiable computation. In 2013 IEEE Symposium on Security and Privacy, pages 238-252. IEEE Computer Society Press, May 2013.

[103] B. Parno, M. Raykova, and V. Vaikuntanathan. How to delegate and verify in public: Verifiable computation from attribute-based encryption. In R. Cramer, editor, TCC 2012, volume 7194 of LNCS, pages 422-439. Springer, Mar. 2012.

[104] A. D. Santis and G. Persiano. Zero-knowledge proofs of knowledge without interaction (extended abstract). In 33rd Annual Symposium on Foundations of Computer Science, Pittsburgh, Pennsylvania, USA, 24-27 October 1992, pages 427-436, 1992.

[105] E. B. Sasson, A. Chiesa, C. Garman, M. Green, I. Miers, E. Tromer, and M. Virza. Zerocash: Decentralized anonymous payments from bitcoin. In Security and Privacy (SP), 2014 IEEE Symposium on, pages 459-474. IEEE, 2014.

[106] J. T. Schwartz. Fast probabilistic algorithms for verification of polynomial identities. Journal of the ACM, 27:701-717, 1980.

[107] H. Shacham and B. Waters. Compact proofs of retrievability. In J. Pieprzyk, editor, ASIACRYPT 2008, volume 5350 of LNCS, pages 90-107. Springer, Dec. 2008.

[108] H. Shacham and B. Waters. Compact proofs of retrievability. In International Conference on the Theory and Application of Cryptology and Information Security, pages 90-107. Springer, 2008.

[109] S. Tillich and N. Smart. Circuits of basic functions suitable for mpc and fhe, 2016. 
[110] F. Tramer, F. Zhang, H. Lin, J.-P. Hubaux, A. Juels, and E. Shi. Sealedglass proofs: Using transparent enclaves to prove and sell knowledge. Euro Security and Privacy'17, 2017. To appear.

[111] J. van der Hoeven and G. Lecerf. On the bit-complexity of sparse polynomial and series multiplication. Journal of Symbolic Computation, 50:227 $254,2013$.

[112] X. Wang, A. J. Malozemoff, and J. Katz. Faster secure two-party computation in the single-execution setting. In Annual International Conference on the Theory and Applications of Cryptographic Techniques, pages 399-424. Springer, 2017.

[113] B. R. Waters. Efficient identity-based encryption without random oracles. In R. Cramer, editor, EUROCRYPT 2005, volume 3494 of LNCS, pages 114-127. Springer, May 2005.

[114] M. N. Wegman and J. L. Carter. New hash functions and their use in authentication and set equality. Journal of computer and system sciences, 22(3):265-279, 1981.

[115] G. Wood. Ethereum: A secure decentralised generalised transaction ledger, 2014.

[116] S. Yamada, G. Hanaoka, and N. Kunihiro. Two-dimensional representation of cover free families and its applications: Short signatures and more. In O. Dunkelman, editor, CT-RSA 2012, volume 7178 of $L N C S$, pages 260277. Springer, Feb. / Mar. 2012.

[117] A. C. Yao. Protocols for secure computations. In Foundations of Computer Science, 1982. SFCS'08. 23rd Annual Symposium on, pages 160-164. IEEE, 1982.

[118] R. Zippel. Probabilistic algorithms for sparse polynomials. E. W. Ng, EUROSM 79, 72:216-226, 1979. 